\title{
WATER-RESOURCES OVERVIEW OF THE MISSISSIPPI GULF COAST AREA
}

by

B. E. Colson and E. H. Boswell

Hydrologists

U. S. Geological Survey

U. S. GEOLOGICAL SURVEY

Open-File Report 85-94

Prepared in cooperation with the DEPARTMENT OF THE ARMY

CORPS OF ENGINEERS

MOBILE DISTRICT

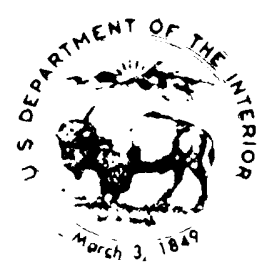

Jackson, Mississippi

1985 


\title{
UNITED STATES DEPARTMENT OF THE INTERIOR
}

\author{
WILLIAM P. CLARK, SECRETARY
}

\section{GEOLOGICAL SURVEY}

\author{
Dallas L. Peck, Director
}

For additional information wrlte to:

District Chiet

U.S. Geological Survey

Water Resources Division

100 West Capltal Street. Sulte 710

Jackson. Mississippl 39269

Telephone: $(601)$ 860-4600
Coples of this report can be purchased from:

Open-Flle Services Section Western Distrlbutlon Branch

U.S. Geological Survey

Box 25425. Federal Center Lakewood. Colorado 80225

Telephone: (303) 234-5888 
Page

Abstract-and

Introduction-_.

Purpose and scope-1.

Physical setting-1.

Water use-_.

Surface water-1.. 8

Principal drainage-1-_. 8

Pascagoula River-........ 8

Tchoutacabouffa River-............ 13

Biloxi River-......... 13

Wolf River-_.-.

Jourdan River............ 16

Pearl River-_._. 19

Surface-water qual ity-_._.

Floods-...

Tidal Records-... 21

Ground water-1

Aquifers-1.

Aquifer hydraulic characteristics-_.

Water-level changes-... 56

Saltwater encroachment_............. 56

Ground-water quality-_. 61

Summary-_-1... 63

Selected references-_._.

Appendix A-Index to surface-water quality data-_......... 69

Appendix B-Summary of ground-water quality data-............ 109

\section{ILLUSTRATIONS}

Figure 1. Map showing area of investigation, geographic

features, and location of gaging and water-quality

stations-.............. 4

2. Geologic map of a part of southern Mississippi-....- 5

3. River basins and hydrologic units in Mississippi

coastal area-.......

Figures 4-9. Duration curves of daily flows,

4. Red Creek at Vestry, 1958-82- 12

5. Escatawpa River near Agricola, 1973-83-........... 14

6. Tuxachanie Creek near Biloxi, 1952-71_.......... 15

7. Biloxi River at Wortham, 1952-82_............. 17

8. Wolf River near Landon, 1971-82_....... 18

9. Pearl River Bogalusa, LA, 1938-83_.............. 20 
10. Index map of the Mississippi gulf coast showing location of quadrangles for which flood boundaries of Hurricane Camille are delineated-_...... 22

11. Index of Federal. Insurance Administration maps-....- 23

12. Density of major water wells in Mississippi gulf coast area-_. 26

13. Geohydrologic section of Hancock County-........- 27

14. Geohydrologic section $A-A^{\prime}$ along Gulf from Pearl

River to Alabama-........ 28

15. Geohydrologic section B-B' from Cat Island to latitude $31^{\circ}$ north-........ 29

16. Geohydrologic section $C-C^{\prime}$ from Horn Island to Greene County-...... 30

17. Citronelle Formation and its relation to underlying

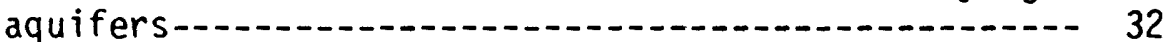

18. Potentiometric surface of the upper part of the Miocene aquifer system, fall 1981, in Jackson, Harrison, and Hancock Counties-................ 34

19. Configuration of the bases of the moderately saline, slightly saline, and frshwater zones in the coastal counties, Mississippi-...-.

20. Hydographs showing water-level changes in representative wells in the Moss Point-Pascagoula

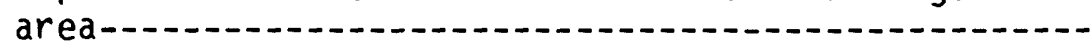

21. Hydrographs showing water-level changes in representative wells in the Biloxi-0cean Spring area 58

22. Hydrographs showing water-level changes in representative wells in the Gulfport area-...... 59

23. Hydrographs showing water-level changes in representative wells in Hancock County........ 60

24. Graph showing depth-temperature relation for ground

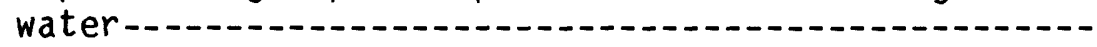

\section{TABLES}

Table 1. Geologic units and their water-bearing properties---- 6

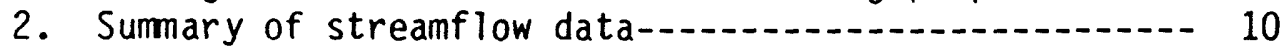

3. Sumary of time-of-travel studies-_._. 11

4. Hurricane tide elevations along the Mississippi

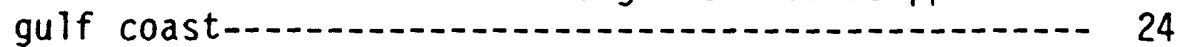

5. Records of selected water wells in Hancock, Harrison and Jackson Counties, Mississippi-_._._. 36

6. Summary of pumping tests in Hancock, Harrison, and Jackson Counties, Mississippi-..... 
WATER-RESOURCES OVERVIEW OF - THE MISSISSIPPI GULF COAST AREA

by B. E. Colson and E. H. Boswell

\begin{abstract}
The Mississippi Gulf Coast region extends from the MississippiLouisiana boundary at Pearl River eastward to the Mississippi-Alabama boundary and includes Hancock, Harrison, and Jackson Counties, Mississippi Sound, and the barrier islands. The region is underlain by southward sloping irregular beds of sand and clay that range from Miocene to Holocene in age.

The Miocene aquifer system in the Gulf Coast area includes waterbearing zones in the Pascagoula and Graham Ferry Formations. The Citronelle aquifer and younger strata overlie the Miocene aquifer system. Freshwater extends to depths ranging from about 1,200 feet east of Pascagoula to slightly more than 3,000 feet in western Hancock County. Most large wells in the area range from 400 to about 1,000 feet in depth and commonly produce 500 to 1,000 gallons per minute.

Water-levels have declined regionally about 2 feet per year during the past 30 years. In the Pascagoula-Moss Point area, concentrated large withdrawals since about 1900 have caused declines of as much as 136 feet. In the Biloxi-Gulfport area declines have been as much as 100 feet from above land surface to about 50 feet below 1 and surface. Although these declines seem large, most of the region can accomodate much larger declines, and the aquifers are capable of yielding much larger quantities of water.
\end{abstract}

The major streams in the region include the Pearl, Jourdan, Wolf, Tchoutachabouffa, Biloxi, Escatawpa, and Pascagoula Rivers. Back Bay of Biloxi and St. Louis Bay are promiment estuarine features. The Pearl and Pascagoula Rivers are the principal drainage from central and southern Mississippi. The Escatawpa River is a tributary to the Pascagoula River. The other rivers drain directly into Mississippi Sound, Back Bay of Biloxi, or St. Louis Bay. The region is subject to flooding from land surface runoff from excessive precipitation and from hurricane-induced flood tides.

Surface water in the region generally is soft, acidic, and low in dissolved solids. Suspended sediment concentrations are low; however, some streams are high in organic color. All streams are subject to saltwater intrusion. 
Ground water is generally of good quality but locally contains excessive concentrations of dissolved solids. Saltwater intrusion has not been observed except in shallow aquifers that are hydraulically connected to estuarine streams. Water-quality problems in deeper aquifers are related to freshwater-sal.twater interfaces that occur in all confined aquifers. Water temperatures range from about $68^{\circ} \mathrm{F}$ in very shallow wells to over $100^{\circ} \mathrm{F}$ in the deepest aquifers.

From Biloxi westward, the deeper aquifers are virtually undeveloped. Fresh surface water is used by industry in the Pascagoula area. Brackish surface water is used in the Biloxi area for thermoelectric cooling. All public water supplies and some industrial water supplies use ground water; however, most of the water used in the area in terms of volume is brackish surface water. Total water use in 1980 was about 50 million gallons per day from ground-water sources and about 720 million gallons per day from surface sources.

\section{INTRODUCTION}

The U.S. Army Corps of Engineers, Mobile District, is making a water-resources management study that includes the three counties that border the Mississippi Gulf Coast. The Corps of Engineers study was authorized by resolutions adopted in June 1972 and March 1973 by the Committees on Public Works of the Senate and House of Representatives, 92d and 93d Congresses of the United States. In 1984, the U.S. Geological Survey entered into agreement with the U.S. Army Corps of Engineers to provide a sumary of available hydrologic information for the area.

\section{PURPOSE AND SCOPE}

This report sumarizes the published and open-file hydrologic information available for the three-county Mississippi Gulf Coast area. The sources of information include the publications and files of the U.S. Geological Survey and other agencies. Surface-water records were run on standard analytical programs to compute statistical relations for low-flow, average flow, and high-flow conditions in major streams. Particular attention was directed to the hydrology of streams at points of entry to the area, and to the problem reaches of the streams within the area. Available publications and file data on ground-water resources are sumarized from the standpoint of recharge areas, delineation of aquifers, ground-water use, changes in water levels, and changes in water quality. No previously unreleased data or interpretative information are presented. 


\section{PHYSICAL SETTING}

The Mississippi Gulf Coast region is bounded on the east by the Mississippi-Alabama state line and on the west by the Pearl River, the common state boundary with Louisiana. The region includes Hancock, Harrison, and Jackson Counties, Mississippi Sound, and the barrier islands situated 10 to 12 miles south of the shoreline. The three counties have a combined area of about 1,800 $\mathrm{mi}^{2}$. The Mississippi Sound comprises about $800 \mathrm{mi}^{2}$ of additional area. The four principal islands, subject to changes in area, include a total of about $50 \mathrm{mi}^{2}$ (fig. 1).

The climate of the study area is characterized by heavy rainfall, hot summers, and mild winters. Rainfall averages about 60 inches annually, and relative humidity generally remains high throughout the year. Temperatures during the sumer months seldom exceed $100^{\circ}$ but often reach $90^{\circ}$ (Newcome and others, 1968).

Land forms in the coastal area, described in detail by Brown and others (1944, p. 17-31), include the Long Leaf Pine Hills, the Coastal Pine Meadows, the alluvial plains of the larger streams, beach ridges, sand dunes, and barrier islands.

The beds exposed at the surface in the area range in age from Miocene to Holocene (fig. 2 and table 1) The area is underlain by a south-southwestward dipping series of deltaic and estuarine sediments that are composed mostly of clay, silt, sand, and irregular beds of gravel. The oldest exposed strata have been assigned to the Pascagoula Formation and are Miocene in age whereas the younger Graham Ferry and Citronelle Formations were considered to be of Pliocene age in Mississippi by Newcome and others (1968).

The Citronelle is a blanket deposit that overlies both the Graham Ferry and the Pascagoula and extends beyond the study area to the north (Boswell, 1979). The citronelle in the coastal area is overlain by Pleistocene and Holocene coastal and terrace deposits and alluvium.

The south-southwestward slope that is characteristic of the older units is the result of gradual subsidence (sinking) in the Gulf Coast geosyncline. The younger beds exhibit successively less southwestward slope. Most of the units thicken down the dip to the southwest (Brown and others, 1944).

The major streams in the area (fig. 1) include the Pearl, Jourdan, Wolf, Tchoutachabouffa, Biloxi, Escatawpa, and Pascagoula Rivers. These streams have well-defined inland channels that provide drainage for the mostly rural area. Back Bay of Biloxi and St. Louis Bay are large estuarine features.

Major population centers within the area of investigation include Pascagoula-Moss Point, Biloxi-0cean Springs, Gulfport-Long Beach-Pass Christian and Bay St. Louis-Waveland. Also included is the National Space Technology Laboratories area, Keesler Air Force Base, and U.S. Naval Construction Battalion Center. 

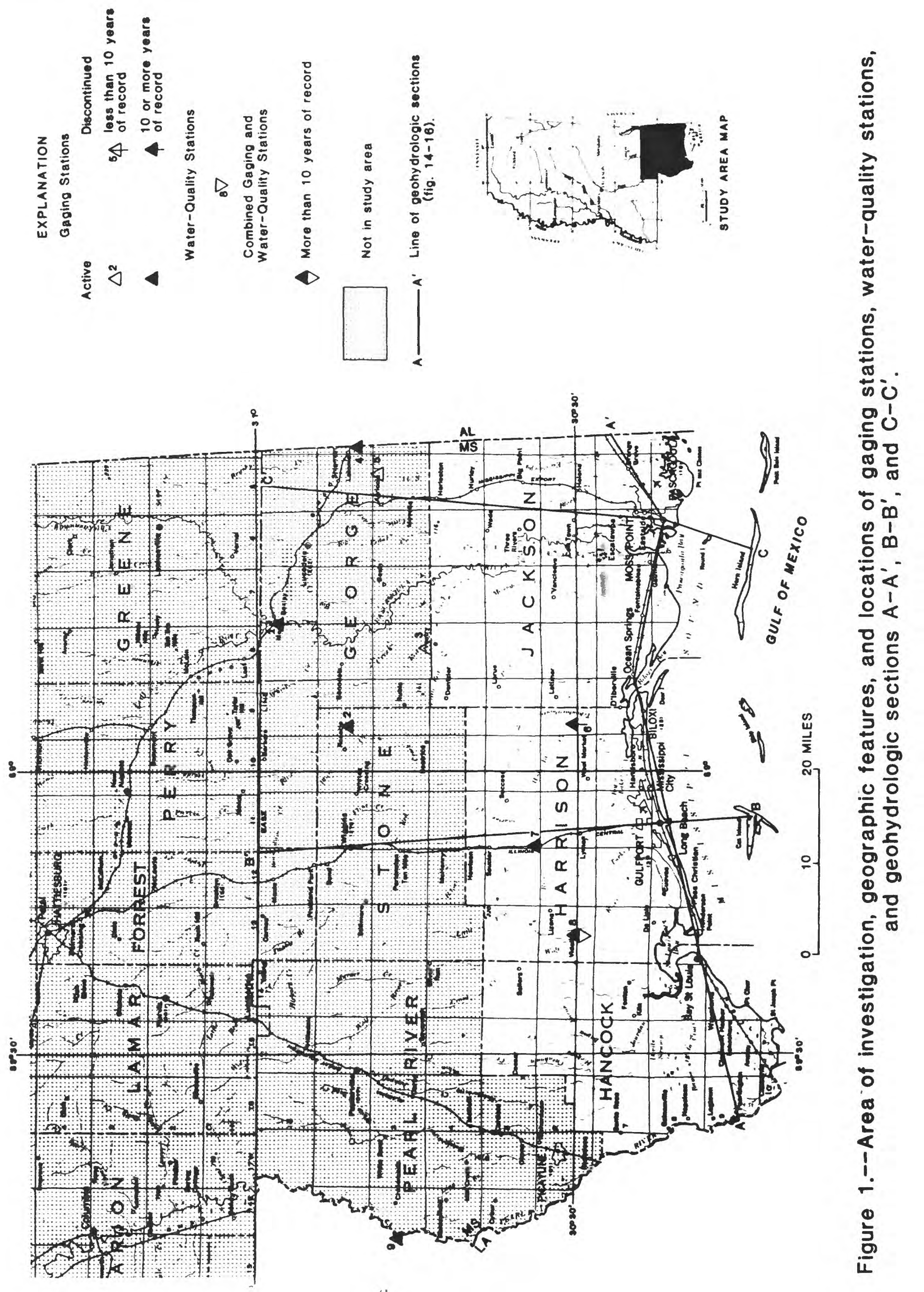

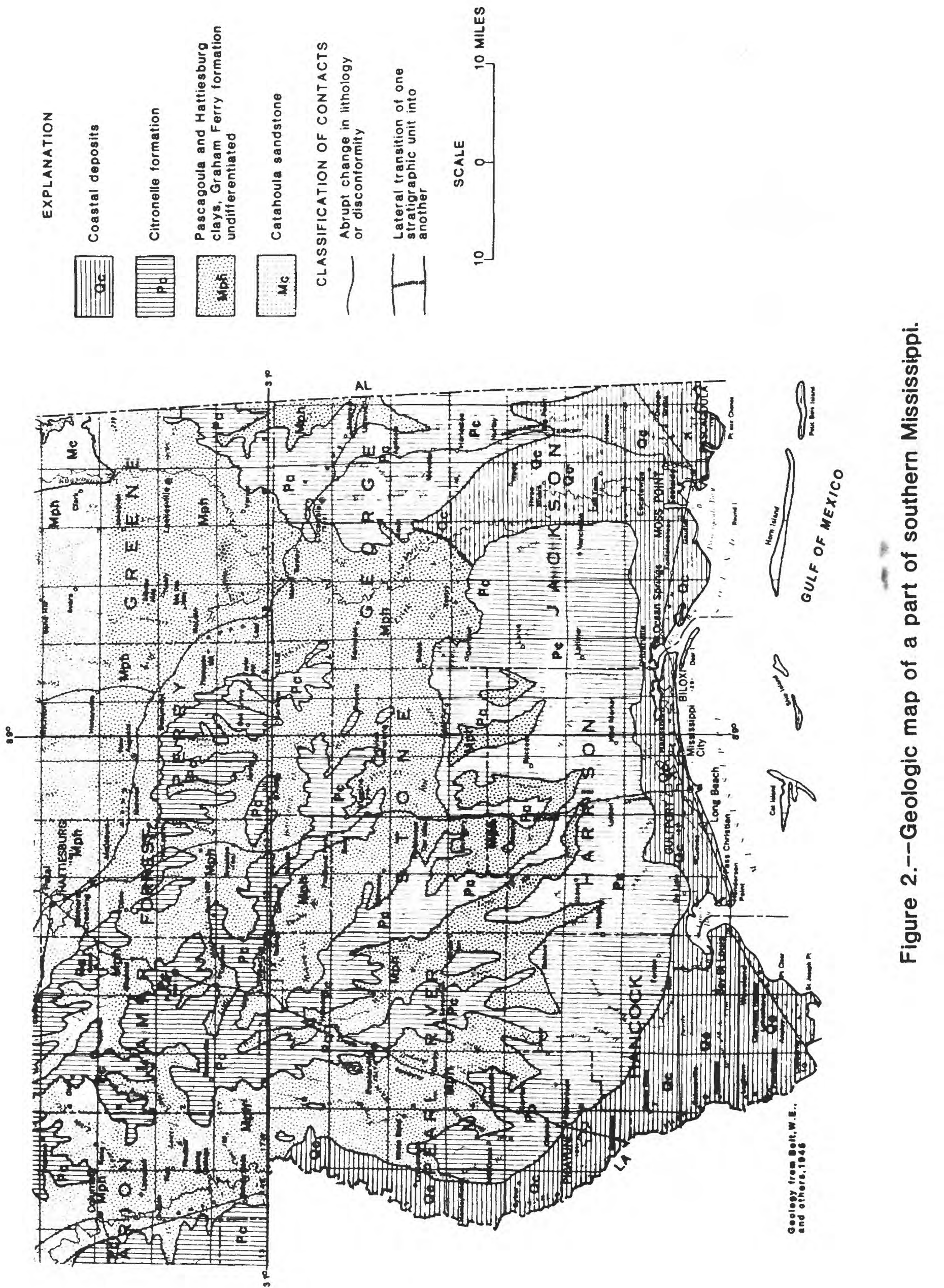
TABLE 1. - Geologio Units and this water-bearing properties

(modified from Harvey, Golden, and Jeffery, 1965, Table 19)

\begin{tabular}{|c|c|c|c|c|c|}
\hline System & Sertes & $\begin{array}{c}\text { Fonma- } \\
\text { tion }\end{array}$ & $\begin{array}{l}\text { Thick- } \\
\text { ness } \\
\text { (feet) }\end{array}$ & $\begin{array}{l}\text { L1thology } \\
\text { and stratigraphy }\end{array}$ & Hydrology \\
\hline & 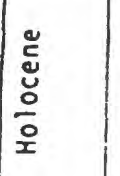 & $\frac{5}{3}$ & 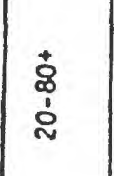 & $\begin{array}{l}\mathrm{Clay}, \text { s1lt, sand and fine } \\
\text { gravel. }\end{array}$ & $\begin{array}{l}\text { Contains water that is } \\
\text { probably salty jas far } \\
\text { north as salt water } \\
\text { penetrates up the } \\
\text { rivers. }\end{array}$ \\
\hline$\stackrel{2}{3}$ & $\frac{\stackrel{2}{c}}{\stackrel{c}{0}}$ & 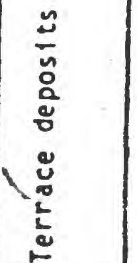 & $\frac{8}{0}$ & $\begin{array}{l}\text { Sand and clay grading } \\
\text { downward into coarse } \\
\text { sand and fine gravel. }\end{array}$ & $\begin{array}{l}\text { Contain fresh water having } \\
\text { a low dissolved-solids } \\
\text { content. Ilear the coast } \\
\text { at shallow depths, the } \\
\text { water is subject to salt- } \\
\text { water encroachment. }\end{array}$ \\
\hline \multirow{5}{*}{$\begin{array}{c}\frac{1}{2} \\
\frac{0}{2} \\
\vdots \\
\vdots\end{array}$} & \multirow[b]{2}{*}{ 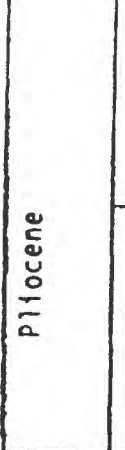 } & $\begin{array}{l}\frac{a}{ \pm} \\
\frac{c}{0} \\
\frac{ \pm}{0}\end{array}$ & $\frac{+}{8}$ & Sand and gravel. & $\begin{array}{l}\text { Maintains high base flows } \\
\text { of streams, and a source } \\
\text { of recharge to the Mio- } \\
\text { cene aquifer system. } \\
\text { Supplies most rural wells } \\
\text { in uplands. }\end{array}$ \\
\hline & & 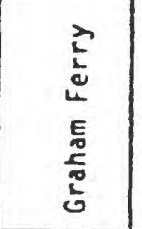 & 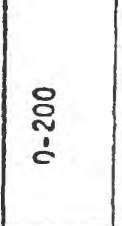 & $\begin{array}{l}\text { Gray, carbonaceous, and } \\
\text { fossillferous clay and } \\
\text { lenticular sand, in } \\
\text { places coarse but us- } \\
\text { ually fine to medium. }\end{array}$ & $\begin{array}{l}\text { Supplies } 60 \text { percent of the } \\
\text { municipal and industrial } \\
\text { ground-water supply. } \\
\text { soft sodium bicarbonate } \\
\text { type of water. }\end{array}$ \\
\hline & \multirow{3}{*}{ 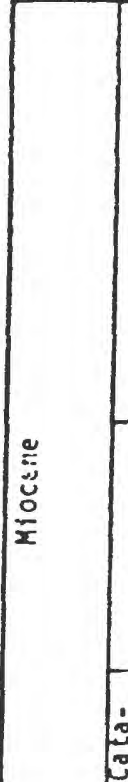 } & $\begin{array}{l}\frac{0}{3} \\
\frac{0}{0} \\
0 \\
0 \\
0 \\
a\end{array}$ & $\begin{array}{l}5 \\
\text { ठ } \\
\frac{1}{\sim} \\
\stackrel{0}{\sim}\end{array}$ & $\begin{array}{l}\text { Clay, shale, and sand. } \\
\text { Sand is lenticular. fine } \\
\text { to very coarse. } \\
\text {. }\end{array}$ & $\begin{array}{l}\text { Comprises several aquifers } \\
\text { along the cgast and many } \\
\text { sand beds of local extent. } \\
\text { The base of fresh water is } \\
\text { in the lower part of the } \\
\text { formation. ithere the thick- } \\
\text { ness is substantial. trans- } \\
\text { misstufty is high. Soft. } \\
\text { sodium bicarbonate type of } \\
\text { water. usually having higher } \\
\text { chloride content than firaham } \\
\text { Ferry Fomation. }\end{array}$ \\
\hline & & $\begin{array}{l}0 \\
5 \\
0 \\
0 \\
0 \\
\vdots \\
\vdots \\
0 \\
\\
2\end{array}$ & 苍 & $\begin{array}{l}\mathrm{Clay} \text { and sand simflar to } \\
\text { Pascagoula Formation. }\end{array}$ & $\begin{array}{l}\text { Contains supplies of fresh } \\
\text { water in counties north of } \\
\text { coastal area. }\end{array}$ \\
\hline & & 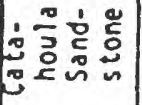 & ஜ्户 & $\begin{array}{l}\text { Sand, shalq, and sand- } \\
\text { stone. }\end{array}$ & Lnused. Saline water. \\
\hline
\end{tabular}


WATER USE

All domestic and public water-supply systems use ground water. Most of the ground-water withdrawals in the region are concentrated in a belt a few miles wide that extends from Wavelarid to Pascagoula along the shoreline of Mississippi Sound.

According to Callahan (1982), most of the water used in the area; in terms of volume, is surface water. Fresh surface water from the Pascaoula River supplies the Bayou Cassotte Industrial area. Freshwater from the Escatawpa River and brackish water from Back Bay of Biloxi is used for cooling at two electric power plants.

The counties along the Mississippi Gulf Coast have experienced an increase in population since 1975, which is reflected in a corresponding increase in water use for some categories. The population served by public water systems increased from 226,655 in 1975 to 252,890 in 1980 , a 12 percent change. A comparison of the 1975 and 1980 water-use totals in million gallons per day for the area is as follows (Callahan, 1982).

\begin{tabular}{lcrrr} 
& \multicolumn{1975}{c}{} & $\underline{1980}$ & $\begin{array}{r}\text { Percent } \\
\text { Change }\end{array}$ \\
\cline { 3 - 5 } Public supplies & $27.57 \mathrm{Mgal} / \mathrm{d}$ & $31.30 \mathrm{Mgal} / \mathrm{d}$ & +14 \\
Self-supplied industry & & & & \\
ground water & $18.42 \mathrm{Mgal} / \mathrm{d}$ & $17.73 \mathrm{Mgal} / \mathrm{d}$ & -4 \\
fresh surface water & $42.35 \mathrm{Mgal} / \mathrm{d}$ & $56.08 \mathrm{Mgal} / \mathrm{d}$ & +32 \\
brackish surface water & $791.49 \mathrm{Mgal} / \mathrm{d}$ & $661.69 \mathrm{Mgal} / \mathrm{d}$ & -16 \\
Rural domestic & $3.65 \mathrm{Mgal} / \mathrm{d}$ & $3.64 \mathrm{Mgal} / \mathrm{d}$ & 0
\end{tabular}

Water use in the three counties in 1980 averaged about $770 \mathrm{Mgal} / \mathrm{d}$ of which 86 percent was brackish surface water used for industrial purposes in Harrison and Jackson Counties. Freshwater use in the counties averaged about $109 \mathrm{Mgal} / \mathrm{d}$. The largest use of fresh surface water, $56.1 \mathrm{Mgal} / \mathrm{d}$, was for industrial supplies in Jackson County. The largest use of fresh ground water, $31.3 \mathrm{Mgal} / \mathrm{d}$, was for public water supplies. The use of ground water in Harrison County, $25.8 \mathrm{Mgal} / \mathrm{d}$, was slightly larger than that in Jackson County, and about five times the amount used in Hancock County.

Of the three counties, Harrison County used the largest amount of brackish surface water, but used very little fresh surface water. Hancock County, with a total water use of less than $5 \mathrm{Mgal} / \mathrm{d}$, used very little water in comparison to Harrison and Jackson Counties.

Data published by the U.S. Geological Survey (Callahan, 1975, 1982, 1983) shows that the use of fresh ground water in the three-county area increased about 6 percent between 1975 and 1980. The use of fresh surface water also increased during this period due largely to the increased demand for industrial water in Jackson County. The use of brackish water increased by about $100 \mathrm{Mgal} / \mathrm{d}$ in Jackson County between 1975 and 1980 but decreased by $231 \mathrm{Mgal} / \mathrm{d}$ in Harrison County. 


\section{SURFACE WATER}

The study area includes five drainage basins as shown on the River Basin and Hydrologic Unit Map (fig. 3). Major streams in the area originate in central Mississippi and discharge into the Gulf of Mexico (U. $S$. Geological Survey, 1977).

Streamflow data collected at continuous-record stations (fig. 1) are summarized in table 2. The maximum and minimum discharges for the period of record are tabulated with average annual runoff for streams with 10 or more years of record. Statistical summaries include low-flow frequency data based on 1-, 7-, and 30-day flows for a 10-year recurrence interval and flow duration values for 10,50, and 90 percent exceedance. Locations of the streamflow gaging stations are shown on figure 1. Four gaging stations that are located upstream outside the area are included to provide more complete coverage. The river basins in the three-county area are described separately below. Coastal area flooding and stream water quality are described in later sections.

\section{Principal Drainage}

\section{Pascagoula River}

Surface water resources of Jackson County are dominated by the Pascagoula River and its local tributaries. The headwaters of the Pascagola River lie in east-central Mississippi and the western edge of Alabama. The Pascagoula flows for about 264 miles and drains a total of $9,498 \mathrm{mi}^{2}$. The average flow at Merrill is $9,873 \mathrm{ft}^{3} / \mathrm{s}$ or $20.35 \mathrm{in} / \mathrm{yr}$ (table 2). The 7-day low flow for a 10-year recurrence is $898 \mathrm{ft} 3 / \mathrm{s}$ and a flow of $1,560 \mathrm{ft}^{3} / \mathrm{s}$ is exceeded 90 percent of the time. The minimum flow since 1930 was $696 \mathrm{ft}^{3} / \mathrm{s}$ on November 1936. The maximum flow at Merrill was $178,000 \mathrm{ft} / \mathrm{s}$ in February 1961, at an elevation of 56.91 feet. National Weather Service information indicated that the flood of April 1900 reached an elevation of 58.7 feet and the flood of July 1916 reached an elevation of 57.2 feet. Time-of-travel studies are shown in table 3 .

Black Creek, with a total drainage area of $1,265 \mathrm{mi}^{2}$, is the largest tributary to the Pascagoula River in Jackson County. Daily discharge records are available since 1971 for Black Creek near Wiggins, Miss., (upstream from the study area) drainage area $701 \mathrm{mi}^{2}$. The average discharge near Wiggins is $1,465 \mathrm{ft} 3 / \mathrm{s}$ and a flow of $2,590 \mathrm{ft}^{3} / \mathrm{s}$ is exceeded 90 percent of the time (table 2). Time-of-travel data are shown in table 3 .

Red Creek (total drainage area, $491 \mathrm{mi}^{2}$ ) is tributary to Black Creek. Daily discharge records are available since 1958 for Red Creek at Vestry, Miss., drainage area $441 \mathrm{mi}^{2}$. The average discharge at Vestry is $856 \mathrm{ft}^{3} / \mathrm{s}$ a flow of $178 \mathrm{ft}^{3} / \mathrm{s}$ is exceeded 90 percent of the time (table 2). Time-of-travel data are shown in table 3 . A duration curve of daily flows for Red Creek at Vestry is shown in figure 4. 


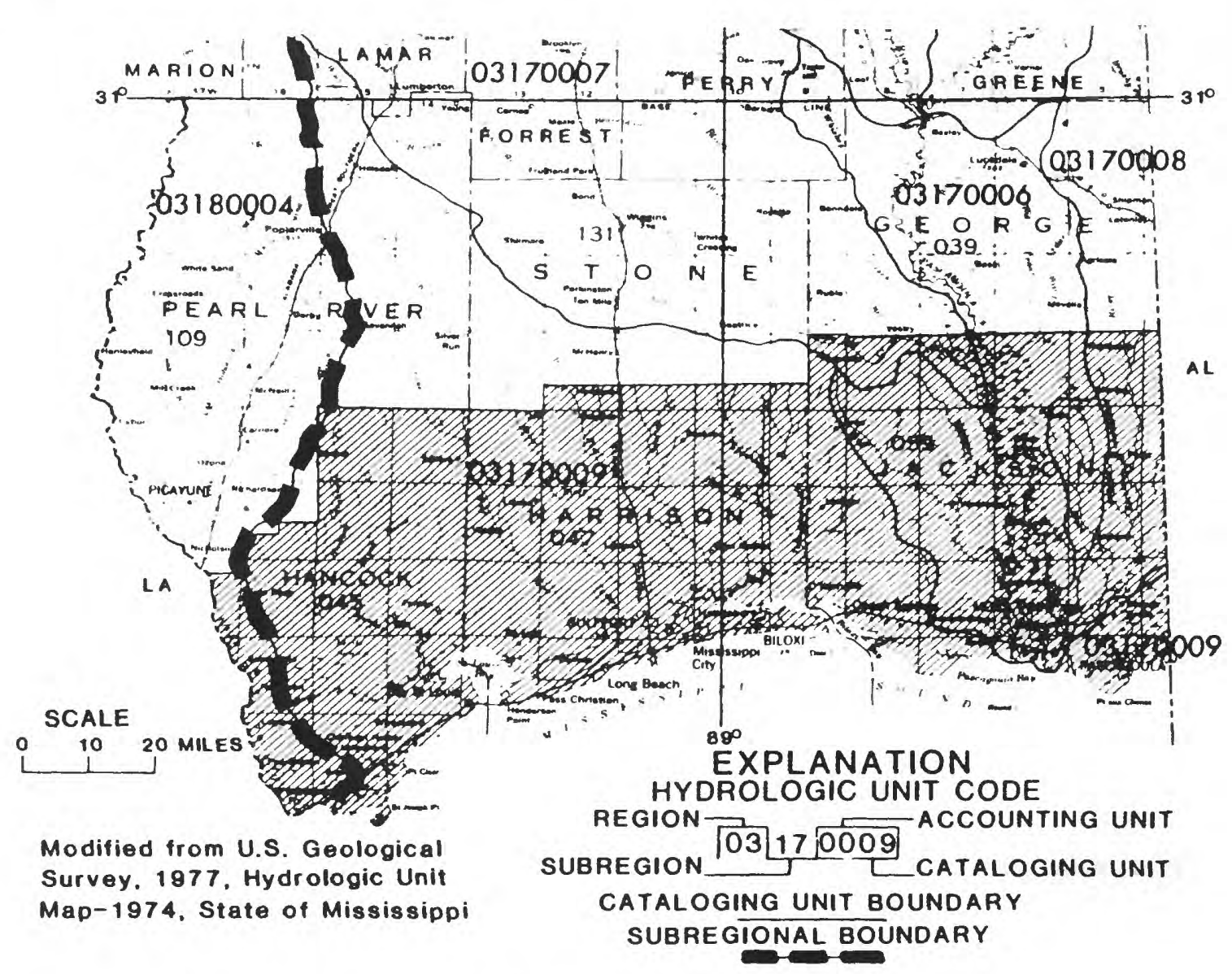

HYOROLOGIC UNITS. MAMES, AND AREAS, IN SRUARE MILES
FOR THE SOUTH ATIAMTIC-GUIF REGION O3 IN MISSISSIPPI

\begin{tabular}{|c|c|c|c|c|c|}
\hline $\begin{array}{l}\text { Sub- } \\
\text { region } \\
\text { No. }\end{array}$ & $\begin{array}{l}\text { Account- } \\
\text { Ing Unit }\end{array}$ & $\begin{array}{l}\text { Cat- } \\
\log 1 \text { ing } \\
\text { Unit }\end{array}$ & Name of Hydrolooic Unit & $\begin{array}{c}\text { Drainage } \\
\text { orea } \\
\text { (so } 1 \text { (1) }\end{array}$ & $\begin{array}{l}\text { valley } \\
\text { length } \\
\text { (mlle) }\end{array}$ \\
\hline \multirow[t]{12}{*}{17} & OD & 06 & Pascagoula River & 9.498 .43 & 264 \\
\hline & & 01 & B) ock Creek & 1.265 .47 & ) \\
\hline & & 08 & Escatampa River & 1.036 .80 & 8 \\
\hline & & 09 & Coastal River basins & & \\
\hline & & & Jourdan River & 390.87 & 41.8 \\
\hline & & & $\begin{array}{l}\text { Bayou LaCrola } \\
\text { Bayou Bacon } \\
\text { HAckory Creek } \\
\text { Catahoula Creek }\end{array}$ & $\begin{array}{r}86.02 \\
41.57 \\
67.27 \\
202.30\end{array}$ & \\
\hline & & & wolf River & 368.33 & 64.0 \\
\hline & & & $\begin{array}{l}\text { Crane Creek } \\
\text { Wurder Creek }\end{array}$ & $\begin{array}{l}41.98 \\
32.26\end{array}$ & \\
\hline & & & Biloxi Alver & 27090 & 39.7 \\
\hline & & & $\begin{array}{l}\text { Litile Blloxt River } \\
\text { Saucter Creek }\end{array}$ & $\begin{array}{l}76.59 \\
48.29\end{array}$ & \\
\hline & & & Tchoutacabouffa River & 24163 & 26.8 \\
\hline & & & Tuxachanie Creek & 94.89 & \\
\hline
\end{tabular}

\begin{abstract}
This map and accompanying table delineate the "river basins" and the "Hydrologic Units" for a part of southern Mississippi. A river basin consists of a drainage system composed of a surface stream or a body of surface water together with all tributary surface streams and bodies of water. A river basin contributes runoff to a stream and is bounded by a drainage Iivide.

A Hydrologic Unit is a geographic area designated as a basis for cataloging and processing the large volumes of hydrologic data and other information. Hydrologic Units depict the basin areal planning units and form a
\end{abstract}

national system for cataloging hydrologic and other information in the National water Data Network. The boundaries of Hydrologic Units coincide with those of river basins but also delineate areas such as intervening segments of drainage areas and islands, estuaries, coastal lands, and other similar areas that are not part of river basins. The Hydrologic unit code consists of an eight digit code representing the Region, Subregion, Accounting, and Cataloging Unit. The Regions, Subregions and Accounting Units are agqregates of the Cataloging Units. The Mississippi coastal area is within the South Atlantic-Gulf Reqion (03) and two subregions (Nos. 17 and 18)

Figure 3.--River basins and hydrologic units in Mississippi coastal area. 


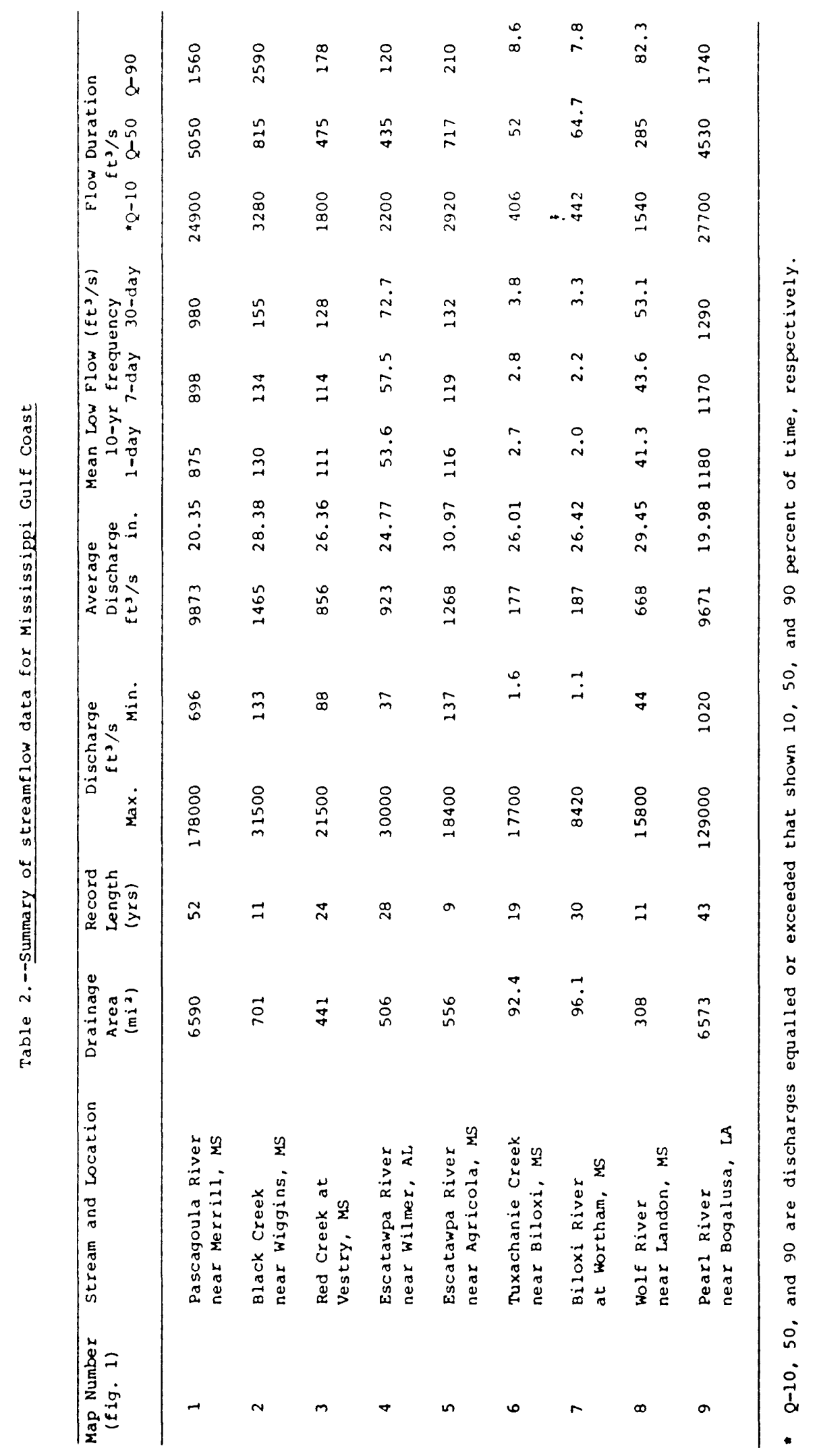




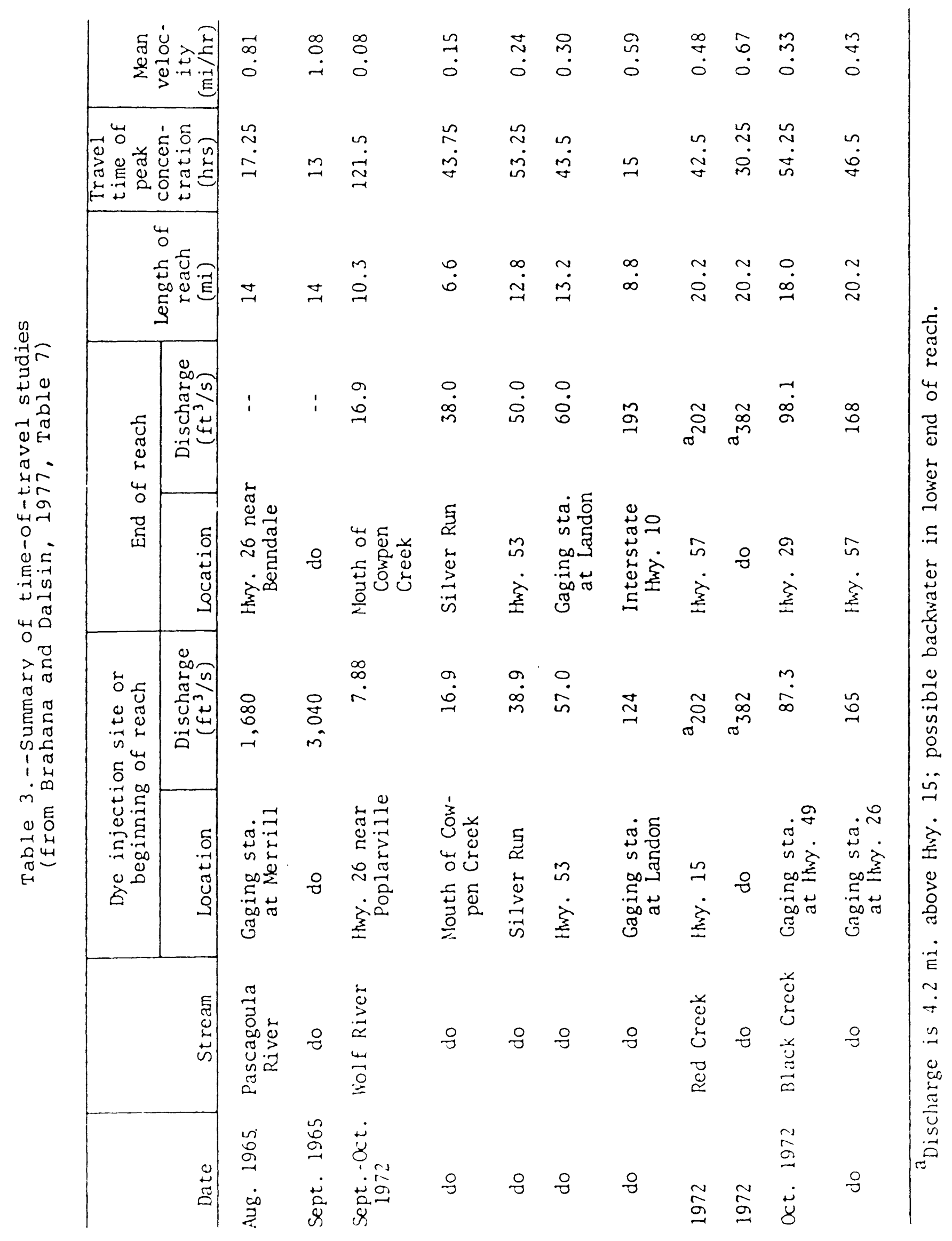




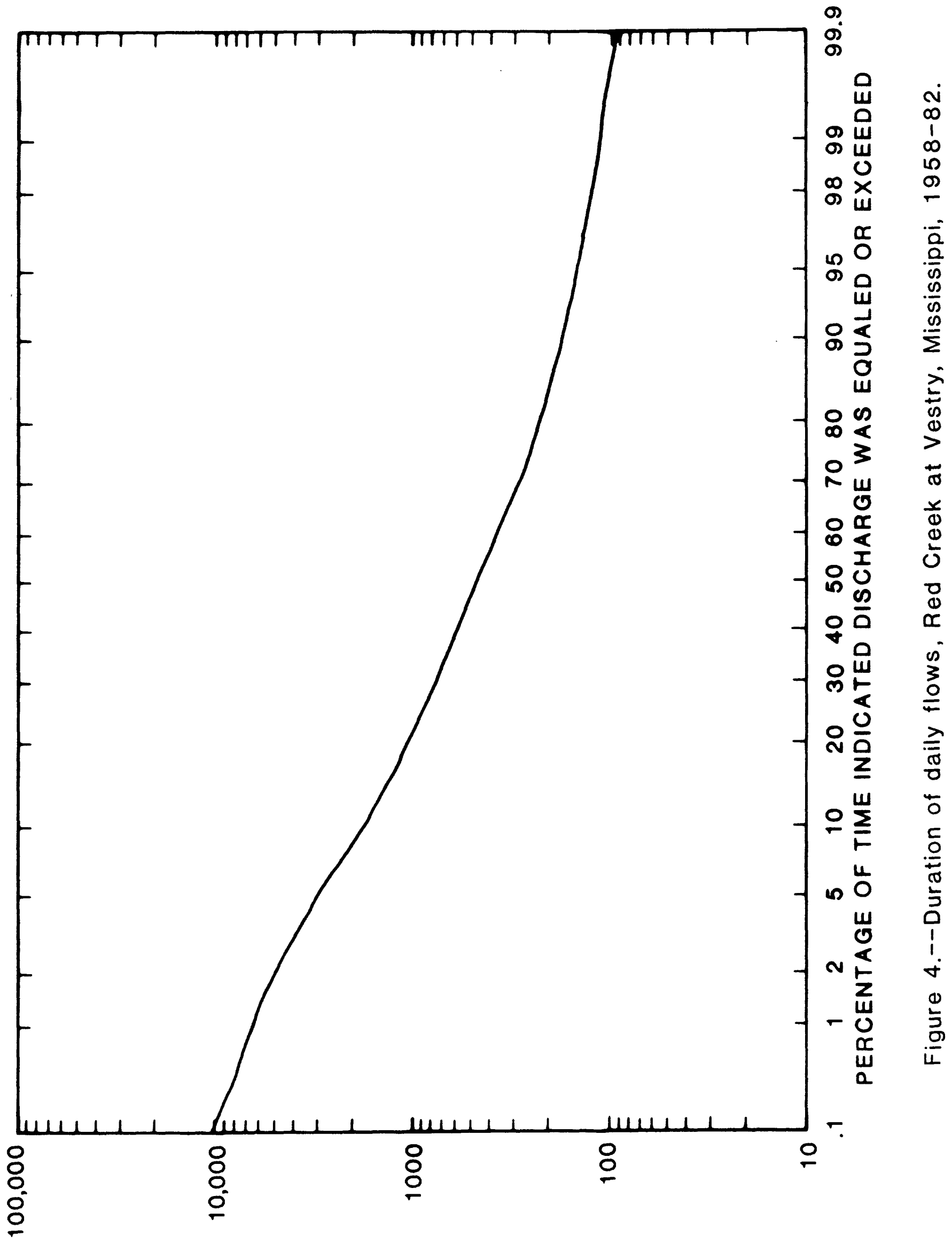

aNOJ 
Escatawpa River (total drainage area, $1,037 \mathrm{mi}^{2}$ ) is tributary to the Pascagoula River. Several large industries are located in the lower part of the mostly rural. Escatawpa River basin. The penetration of saltwater in Escatawpa River was reported 15.5 miles upstream of its mouth on September 16, 1.954 (Harvey and others, 1965). The minimum observed flow of $37 \mathrm{ft} 3 / \mathrm{s}$ occurred in 1954 on Escatawpa River upstream from the study area near Wilmer, Ala., (drainage area, $506 \mathrm{mi}^{2}$ ). The average flow is $923 \mathrm{ft} 3 / \mathrm{s}$ and a flow of $120 \mathrm{ft} 3 / \mathrm{s}$ is exceeded 90 percent of the time near Wilmer (table 2).

Records since 1973 are available for Escatawpa River near Agricola, Miss., (drainage area $556 \mathrm{mi}^{2}$ ). The minimum observed flow of $137 \mathrm{ft} / \mathrm{s}$ occurred in 1978. The average flow is $1,268 \mathrm{ft}^{3} / \mathrm{s}$, and flow of $210 \mathrm{ft} 3 / \mathrm{s}$ is exceeded 90 percent of the time (table 2). A duration curve of daily flows for Escatawpa River near Agricola for the period 1973-83 is shown in figure 5. The maximum known flood reached an elevation of 72 feet in March 1929 on the gage near Agricola.

\section{Tchoutacabouffa River}

The eastern part of Harrison County and the western edge of Jackson County are drained by the Tchoutacabouffa River. The Tchoutacabouffa River basin, which contains $242 \mathrm{mi}^{2}$, is mostly rural and 1 ies primarily in the De Soto National Forest. The Tchoutacabouffa River flows south to a point just north of D'Iberville and then turns southwestward to enter the Back Bay of Biloxi near the mouth of Biloxi River. The principal tributary of Tchoutacabouffa River is Tuxachanie Creek (drainage area, $94.9 \mathrm{mi}^{2}$ ). Other tributaries include Hurricane Creek, Bayou Billie, and Bayou Costapia.

The drainage area of Tuxachanie Creek is $92.4 \mathrm{mi}^{2}$ at State Highway 15 near Biloxi. The channel is about 26 miles long upstream of State Highway 15. The slope between points 10 and 85 percentile of the 26-mile distance is about 7 feet per mile. The average discharge of Tuxachanie Creek at State Highway 15 near Biloxi is $177 \mathrm{ft}^{3} / \mathrm{s}$ (table 2). The 7-day low flow for a 10-year recurrence is $2.8 \mathrm{ft} 3 / \mathrm{s}$. The flow in Tuxachanie Creek at State Highway 15 near Biloxi equals or exceeds $8.6 \mathrm{ft}^{3} / \mathrm{s} 90$ percent of the time (table 2). A duration curve of daily flows for Tuxachanie Creek near Biloxi is shown in figure 6 .

\section{Biloxi River}

The Biloxi River along with its two principal tributaries, Little Biloxi (drainage area, $76.6 \mathrm{mi}^{2}$ ) and Saucier Creeks (drainage area, $48.3 \mathrm{mi}^{2}$ ), drain $271 \mathrm{mi}^{2}$ primarily in central Harrison County. The Biloxi River begins at an elevation of about 335 feet about 15 miles north of the Stone-Harrison County line, flows southeast for about 40 miles and discharges into Biloxi Bay (fig. 1). The streambed reaches sea level about 6 miles upstream of the mouth near Lyman.

Zero flow was observed in the Biloxi River at the gage near Lyman $\left(251 \mathrm{mi}^{2}\right.$ ) in 1965 due to tide effect. The minimum discharge observed without tide effect, $41.3 \mathrm{ft} 3 / \mathrm{s}$, occurred in 1966 . The average 


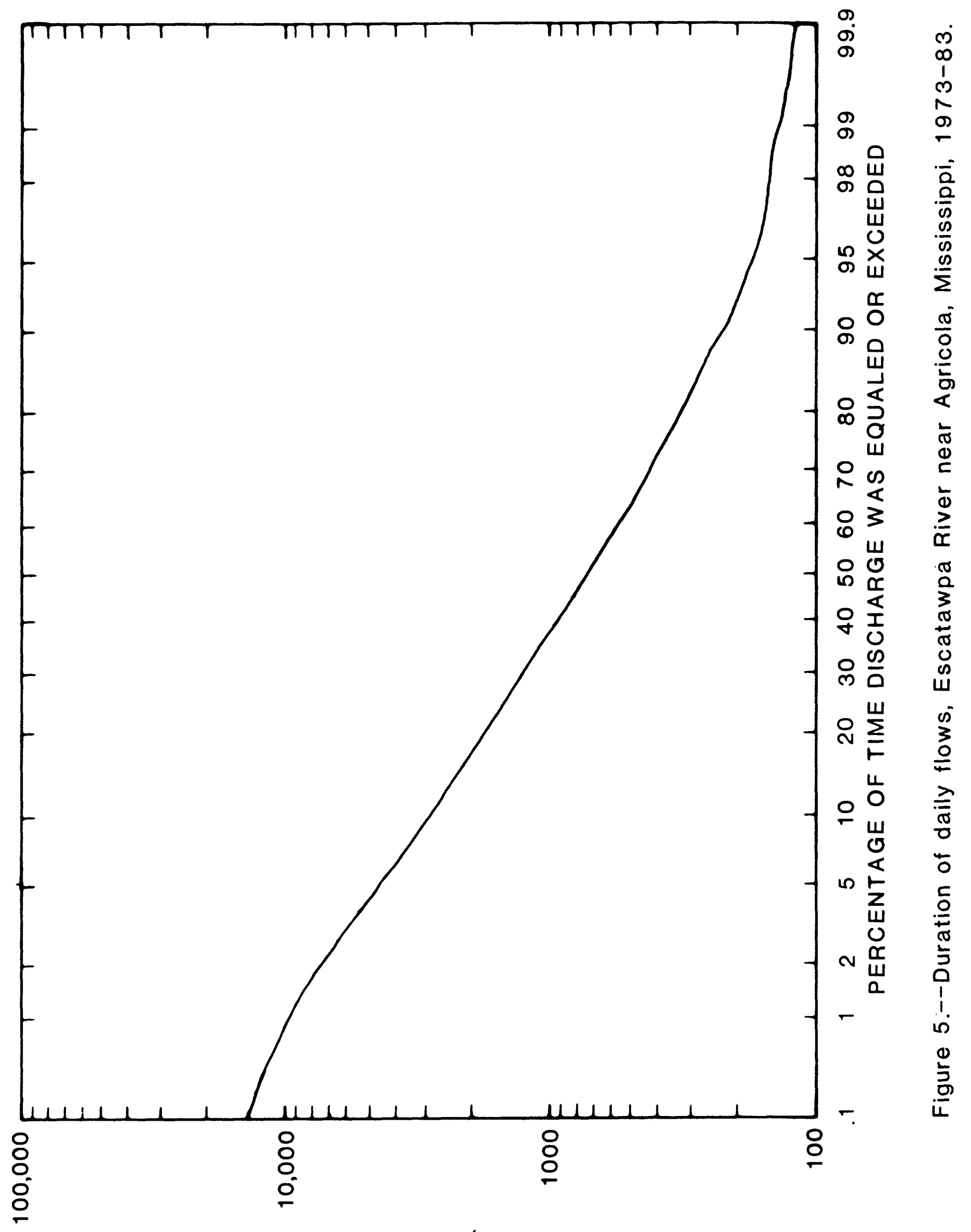

aNOJ 


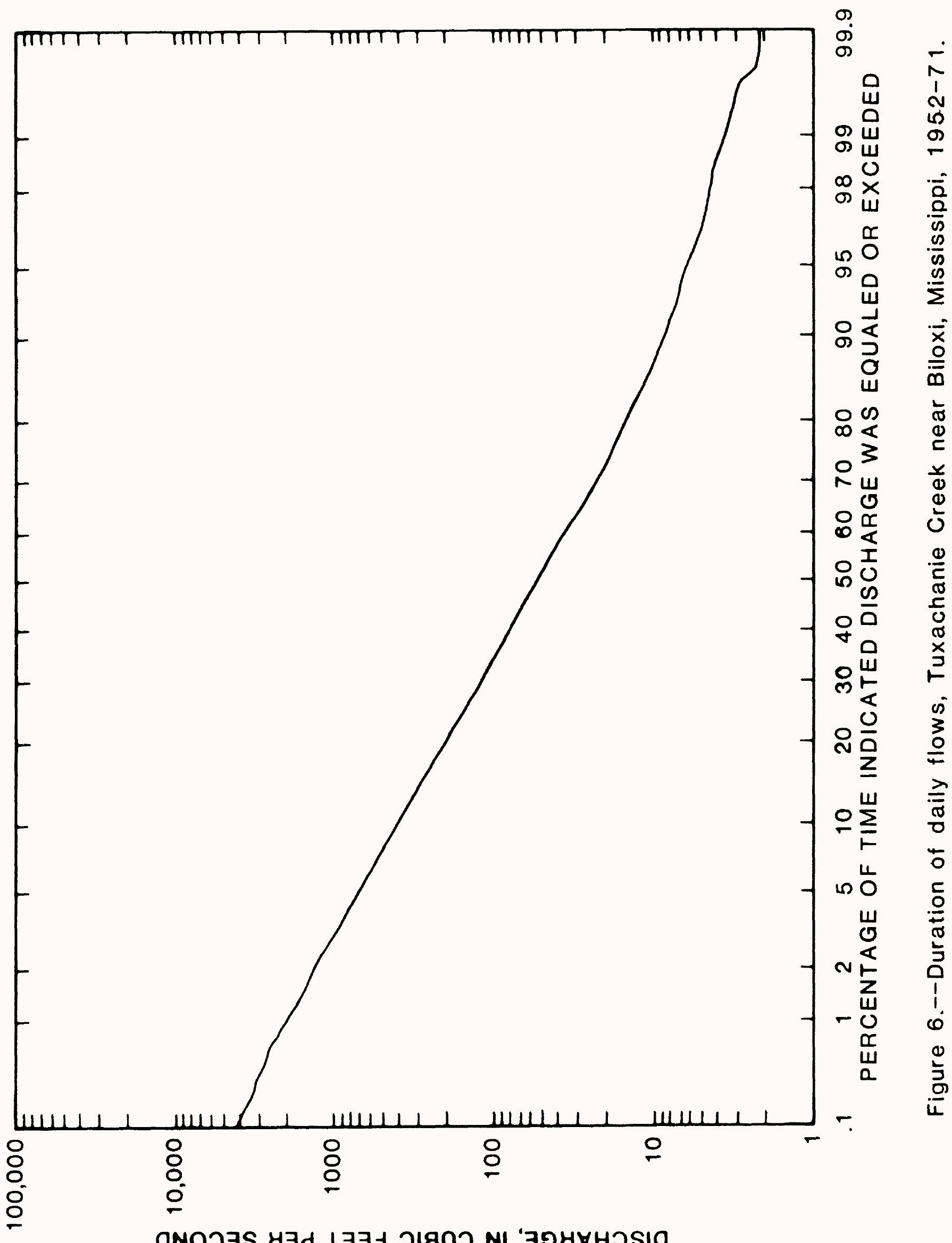

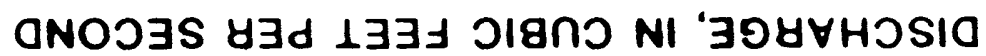


discharge of the Biloxi River at Wortham (96.1 $\left.\mathrm{mi}^{2}\right)$ is $187 \mathrm{ft}^{3} / \mathrm{s}$ (table 2). The minimum discharge observed at Wortham of $1.1 \mathrm{ft} 3 / \mathrm{s}$ occurred on October 21, 1963. "The 7-day. Tow flow at Wortham for a 10-year recurrence is $2.2: \mathrm{ft}^{-3} / \mathrm{s}$ and a flow of $7.8 \mathrm{ft}^{3} / \mathrm{s}$ is exceeded 90 percent of the time. A duration curve of daily flows for Biloxi River at Wortham is shown in figure 7.

The maximum elevation recorded on $B i l o x i$ River at Wortham since 1952 of 42.26 feet occurred on April 27, 1964. The flood of 1948 reached an elevation of 44.5 feet, and from the information by local residents, the floods of 1916 and 1928 reached about the same elevation.

The minimum discharge of $3.1 \mathrm{ft}^{3} / \mathrm{s}$ observed on Saucier Creek near Wortham occurred in 1954. :The minimum discharge of $3.4 \mathrm{ft}^{3} / \mathrm{s}$ observed on Little Biloxi River near Lyman occurred in 1963.

\section{Wolf River}

The Wolf River drains $368 \mathrm{mi}^{2}$ including the western part of Harrison County and the northeastern corner of Hancock County. The main-channel length is about 64 miles from the drainage divide to the mouth in St. Louis Bay. The largest tributary with $42 \mathrm{mi} 2$ is Crane Creek.

Time-of-travel studies on Wolf River conducted in September-0ctober 1972 are shown in table 3. Daily streamflow records are available for the gaging station at State Highway 53 for the periods October 1944 to June 1948 and September 1964 to September 1971 and for the gaging station near Landon from october 1971 to date. The average flow is $668 \mathrm{ft} 3 / \mathrm{s}$ or or $29.45 \mathrm{in} / \mathrm{yr}$ at the site near Landon. The 7-day low flow for a 10-year recurrence interval is $43.6 \mathrm{ft}^{3} / \mathrm{s}$ (table 2); a discharge of $82.3 \mathrm{ft} 3 / \mathrm{s}$ is equaled or exceeded 90 percent of the time. A duration curve of daily flows for Wolf River near Landon, Miss., is shown in figure 8 .

\section{Jourdan River}

Most of Hancock County is drained by the Jourdan River, which flows southeast across the central part of the county. Including Catahoula Creek, the principal tributary, the Jourdan River flows for about 42 miles before emptying into $S t$. Louis Bay (fig. 1). Bayou LaCroix drains Devils Swamp from the west and enters Jourdan River just upstream of St. Louis Bay. The total drainage area of $391 \mathrm{mi}^{2}$ is mostly rural with urban areas concentrated along the coastline near Bay St. Louis, Miss.

Other tributaries to Jourdan River include Bayou Bacon, Rotton Bayou, and Dead Tiger, Mill, and Hickory Creeks. 


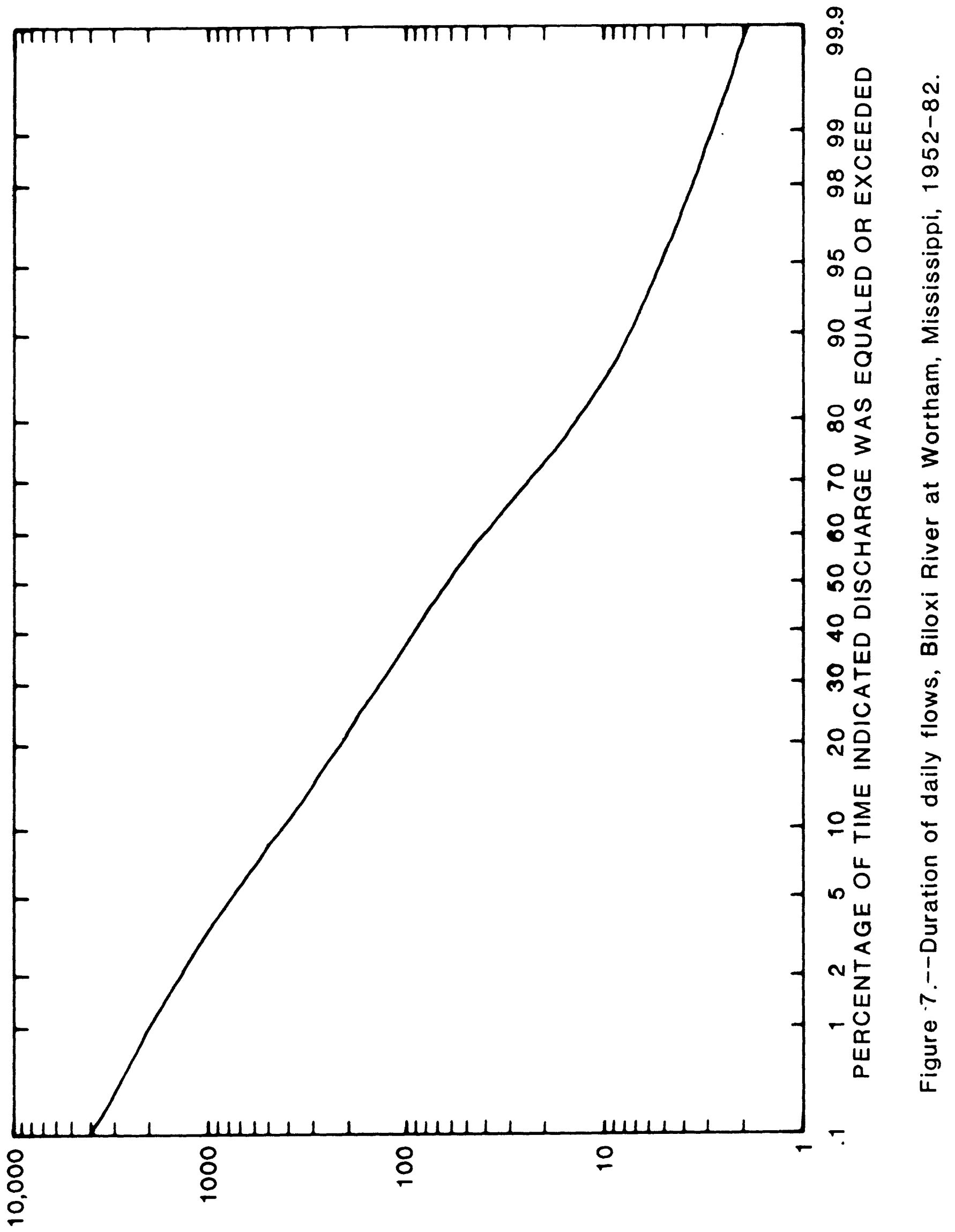

ONOJ 


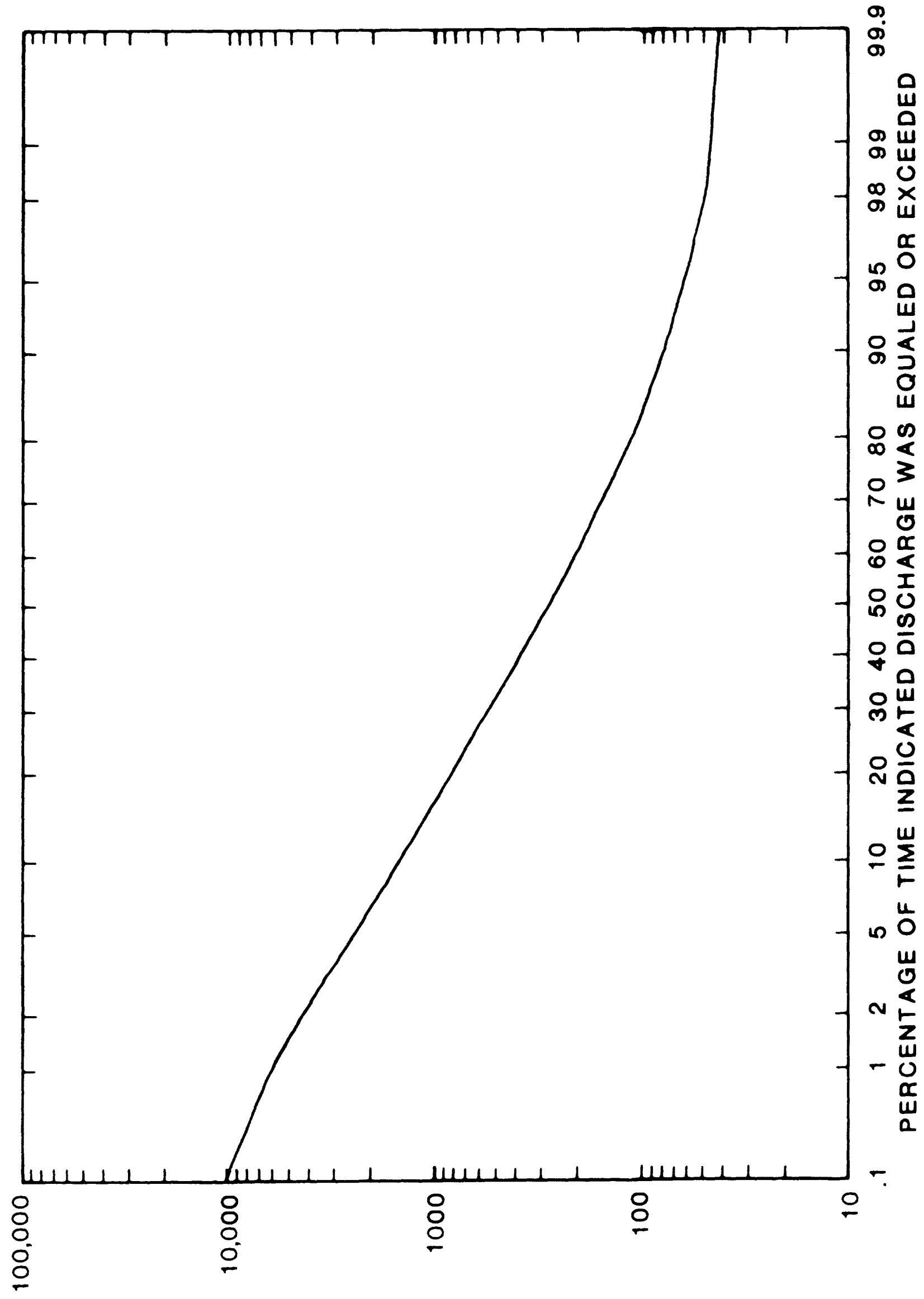

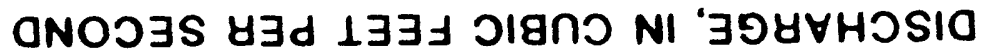




\section{Pearl River}

The Pearl River basin drains $8,674 \mathrm{mi}^{2}$ of which about 7,750 $\mathrm{mi}^{2}$ are in Mississippi and the remainder in Louisiana. The Pear 7 River forms the boundary between Louisiana and Mississippi for about 112 miles.

The most downstream $=$ continuous record station is Pearl River near Bogalusa, La. The drainage area at this site is 6,573 $\mathrm{mi}^{2}$ and the average discharge $9,671 \mathrm{ft}^{3} / \mathrm{s}$ (table 2): The 7-day low flow for a 10-year recurrence is $1,170 \mathrm{ft}^{3} / \mathrm{s}$ and the minimum observed discharge of $1,020 \mathrm{ft}^{3} / \mathrm{s}$ occurred in 1963. A duration curve of daily flows for Pearl River near Bogalusa is shown in figure 9.:

\section{Surface-Water Quality}

Streamflow and water-quality data are obtained by the U.S. Geological Survey from a network of sites (fig. 1). The U.S. Geological Survey currentiy operates two continuous-record stations and four partial-record stations in the area. Specific hydrologic information has been collected at numerous sites in the three-county area as a result of various projects. An index to water-quality data available from the U.S. Geological Survey is given in Appendix A.

Surface waters in the streams of the area are generally suitable for most uses. Chemical analyses indicate that the water in freshwater streams is generally soft, acidic ( 5.0 to $7.0 \mathrm{pH}$ units), with low concentrations of dissolved solids. Hardness is usually less than $50 \mathrm{mg} / \mathrm{L}$ and the dissolved-solids concentrations less than $100 \mathrm{mg} / \mathrm{L}$. The concentrations of dissolved oxygen are usually greater than $4 \mathrm{mg} / \mathrm{L}$, which is sufficient to support a healthy fish population (Brahana and Dalsin, 1977). Discharge of waste into streams increases the dissolved-solids content and decreases the dissolved oxygen depending on the amount and types of waste material. Dissolved solids derived from ground-water discharge increases the dissolved mineral content of streams during low flow periods. Tanic acid, leached from decaying vegetation, is a source of high color in some streams. Suspended-sediment concentrations in streams generally are low but occasionally exceed $100 \mathrm{mg} / \mathrm{L}$ during periods of storm runoff (Newcome and others, 1968).

The movement of saltwater upstream during high tide causes mixing with freshwater and increases the dissolved solids concentrations in estuarine streams. The distance upstream and extent of the increase in dissolved-solids concentrations is dependent on freshwater stream discharge and tide stage. Saltwater intrusion was reported 10 miles upstream of the mouth of Jourdan River on March 5, 1974. Penetration of saltwater in Escatawpa River was reported 15.5 miles upstream of its mouth on September 16, 1954 (Harvey and others, 1965). The streambed of Wolf River is below sea level for at least 10 miles upstream of the mouth (Newcome and others, 1968). There is a potential for saltwater encroachment on all coastal streams where the thalweg is below sea level. 


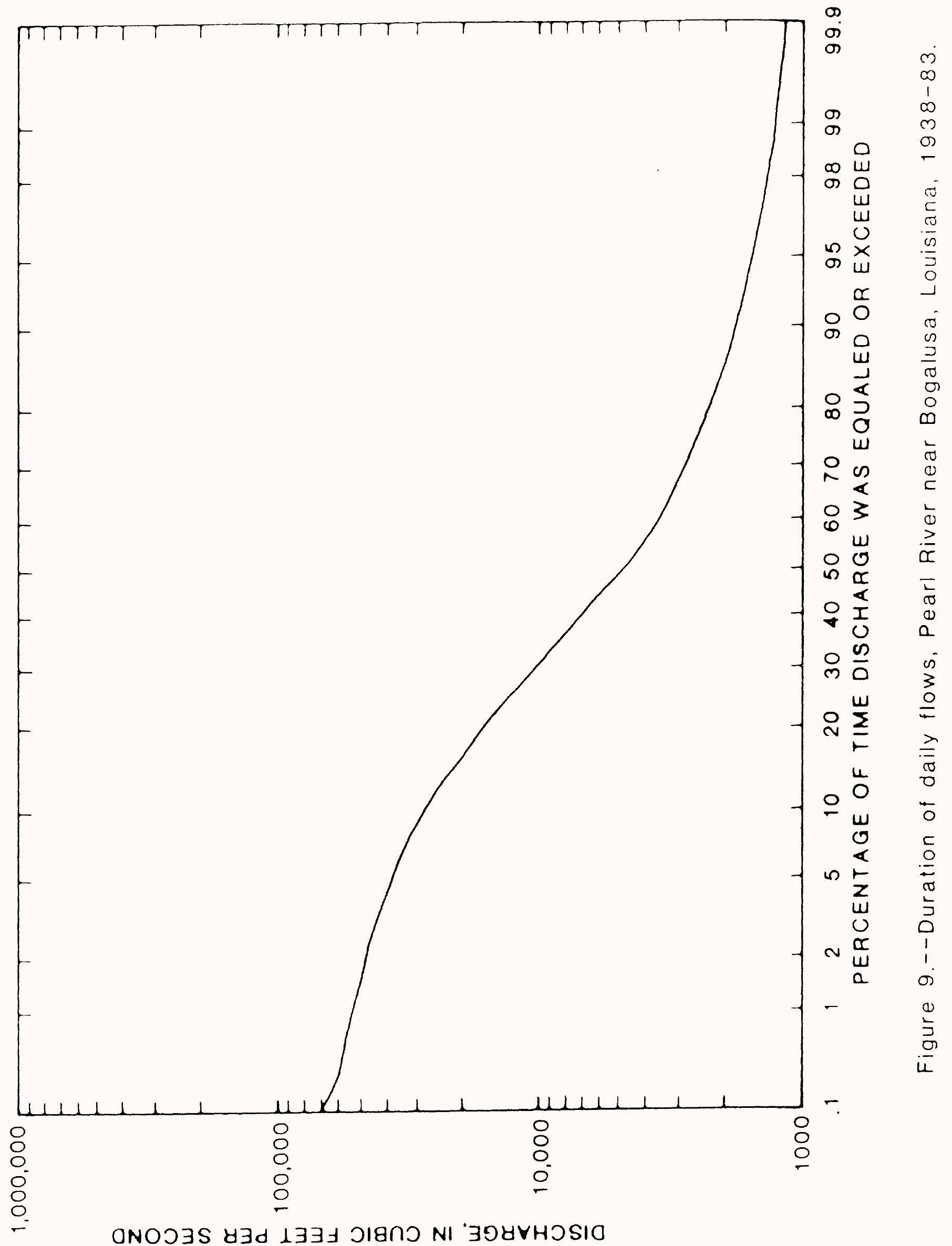




\section{Floods}

The Mississippi Gulf Coast is subject to floods from two distinct sources (1) headwater floods caused by excessive precipitation on the drainage basins and (2) hurricane induced flood tides along the coast. It is beyond the scope of this report to discuss all areas of known flooding. Instead, the intent of this section is to identify some of the flood-plain management activities and flood studies, past, present, and future.

The city and county communities in the three-county area have taken various approaches toward managing flood-prone areas. As a result of Hurricane Camille in August 1969, a series of 12 hydrotogic atlases were prepared by the U.S. Geological Survey that. del ineated Hurricane Camille flood boundaries (fig. 10). Additional data are presented that express the relation of high-tide elevations at Biloxi to the probability of being equaled or exceeded in any year.

All three coastal counties and nine cities are covered by Flood Insurance Administration maps (fig. 11). These maps delineate the 100 -and 500-year flood boundaries. Velocity zones have been delineated along the coastline of the three counties.

Information on peak stages, discharges, and flood statistics is available for the sites of U.S. Geological Survey continuous-recording stations (fig. 1). At other stations that record only peak stages, the U.S. Geological Survey has calculated peak discharges from stagedischarge relationships.

\section{Tidal Records}

Records of storm tides for the Mississippi coast since 1882 have been recorded at Biloxi by the Corps of Engineers and others. A tide gage is located near the center of the Louisville and Nashville Railroad bridge across the Back Bay of Biloxi. Tide gages with much shorter records are operated by the Corps of Engineers at Mobile and Dauphin Island, Ala., and Pascagoula and Pearlington, Miss. Significant tide elevations at various points along the Mississippi coast for more than 20 hurricanes since 1893 have been recorded by the Corps of Engineers, the U.S. Geological Survey, and others. Data pertaining to some of the highest tides of record are shown in table 4. 


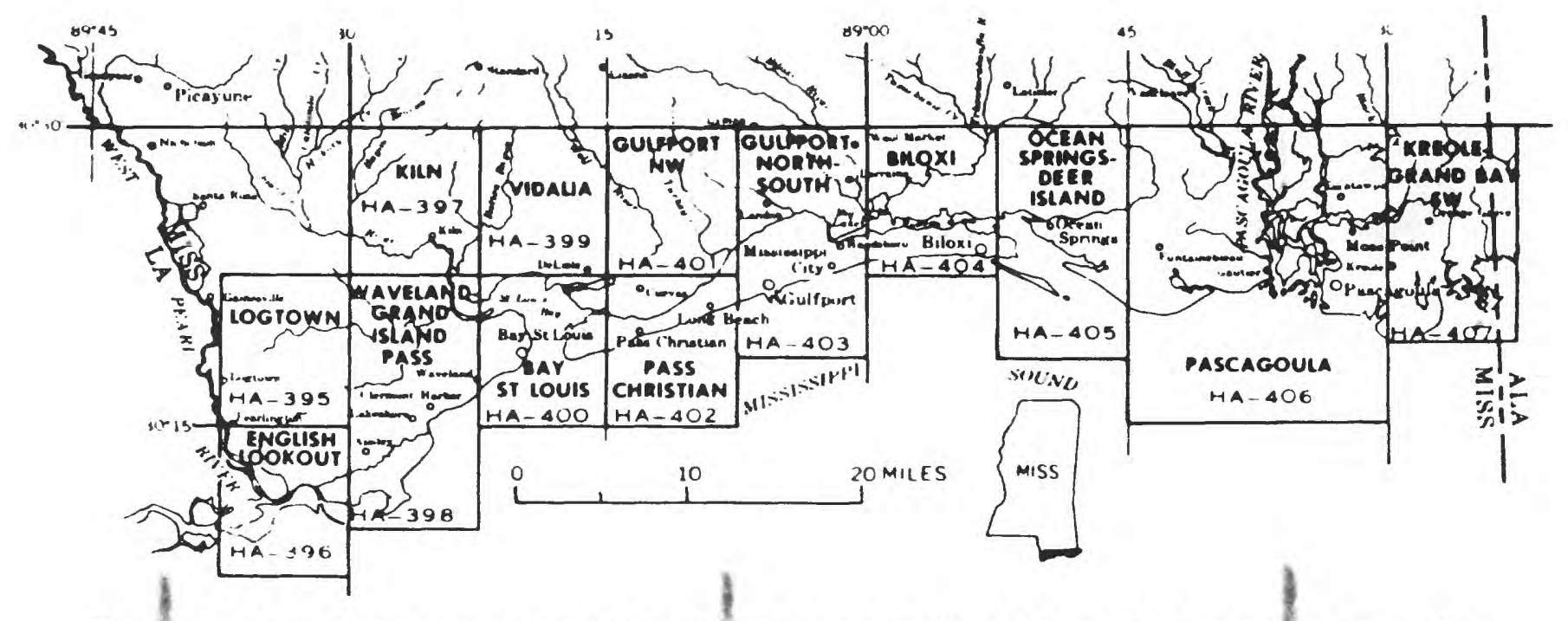

Figure 10.--Index map of the Mississippi Gulf Coast showing location of quadrangles for which flood boundaries of Hurricane Camille are delineated. 


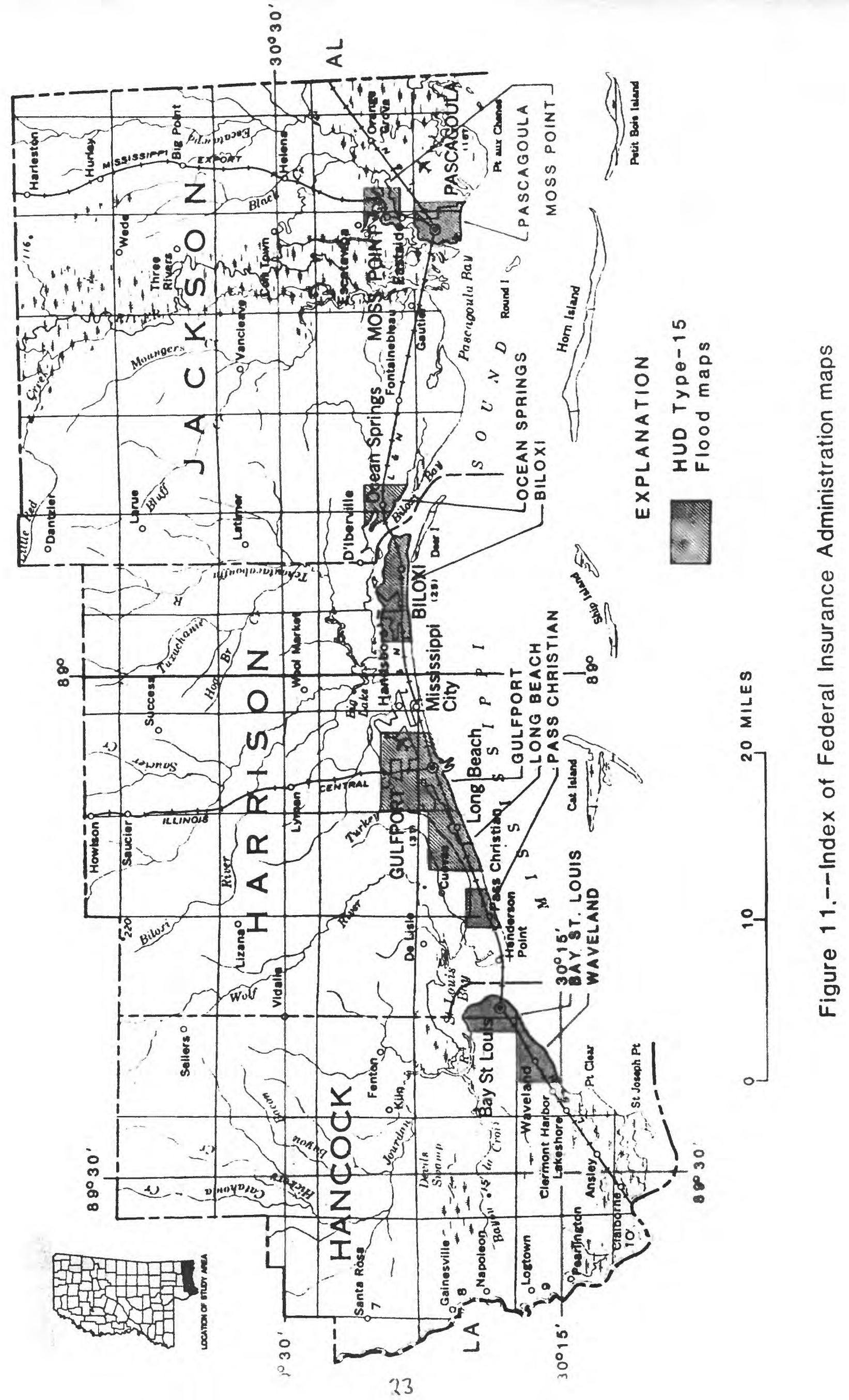




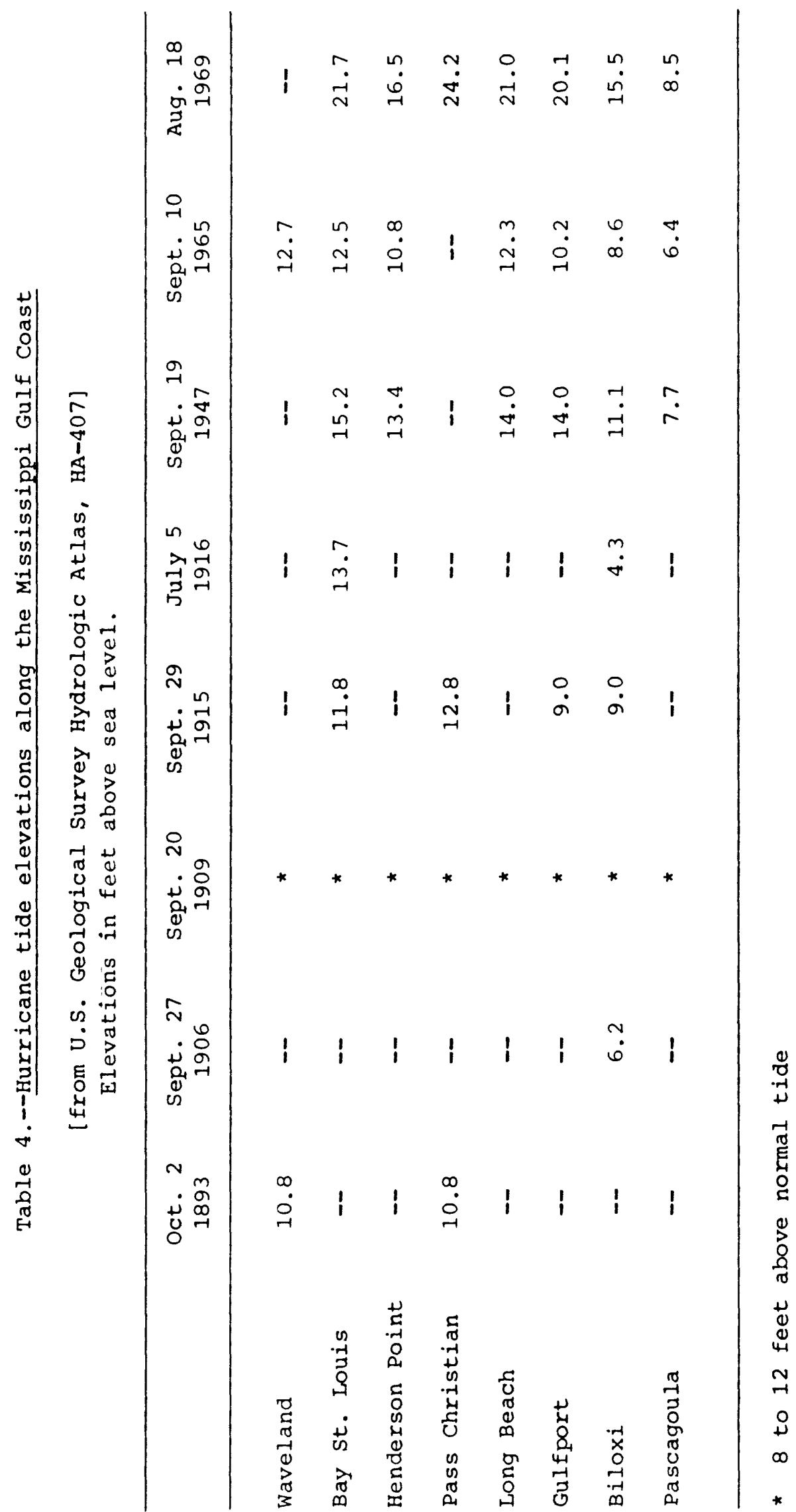




\section{GROUND WATER}

The Mississippi Gulf Coast area is rich in ground-water resources. The first flowing artesian well is reported to have been drilled in 1884 and until recent years the population along the coast had been supplied with water by "flowing wells that had artesian heads as high as 60 to 80 feet above sea level. The drilling firms operating in the coastal area reported to G. F. Brown (1944, p. 66) that a total of 83 wells had been drilled by 1901 and by 1903 there were 119 wells, the deepest of which was 1,550 feet. By: 1979, U.S. Geological Survey files contained records for about 4,200 wells located within about 6 miles of the coastline. In 1984, there are about 5,000 wells and the number continues to increase. A description of majot wells is given in table 5 .

The large number of major wells in the area (fig. 12) reflect the abundance of fresh ground water and the growth of population and industry along the coast. A favorable, aspect of the ground-water resources is quality; many of the wells produce water that requires little or no treatment for general use (Newcome and others, 1968). The availability of ground water is enhanced by the artesian pressures in the deeper aquifers that result in the natural flows or near-surface water levels that persist until today, except in areas where large volumes of water have been withdrawn.

\section{Aquifers}

The south-southwestward slope of the older stratigraphic units is the result of long-term geologic subsidence in the Gulf coast geosyncline. The younger units exhibit successively less southwestward slope, and most of the units thicken down the dip to the southwest (Rainwater, 1962, fig. 25). Sediments are composed mostly of clay, silt, and sand. Irregular gravelly sand beds occur throughout the Miocene and Pliocene age deposits.

The distribution of the ground-water resource is not uniform and the freshwater zone thickens westward from the Alabama line to Hancock County (Brown and others, 1944). The number of major aquifers underlying the coast has not been established, but water-level differences indicate that several separate water-bearing zones probably underlie most of the coastal area. The water-bearing zones are composed of discontinuous sandy beds that are highly irregular in thickness and areal extent. Figure 13 is modified from a geohydrologic section across Hancock Couny (Newcome, 1967a) that demonstrates typical subsurface conditions in the coastal area.

The geohydrologic conditions in the Mississippi coastal area are illustrated in figures 14,15 , and 16 . The relative positions of the water-bearing units and the freshwater-saltwater boundaries are shown schematically and representative water wells show the range of development. 


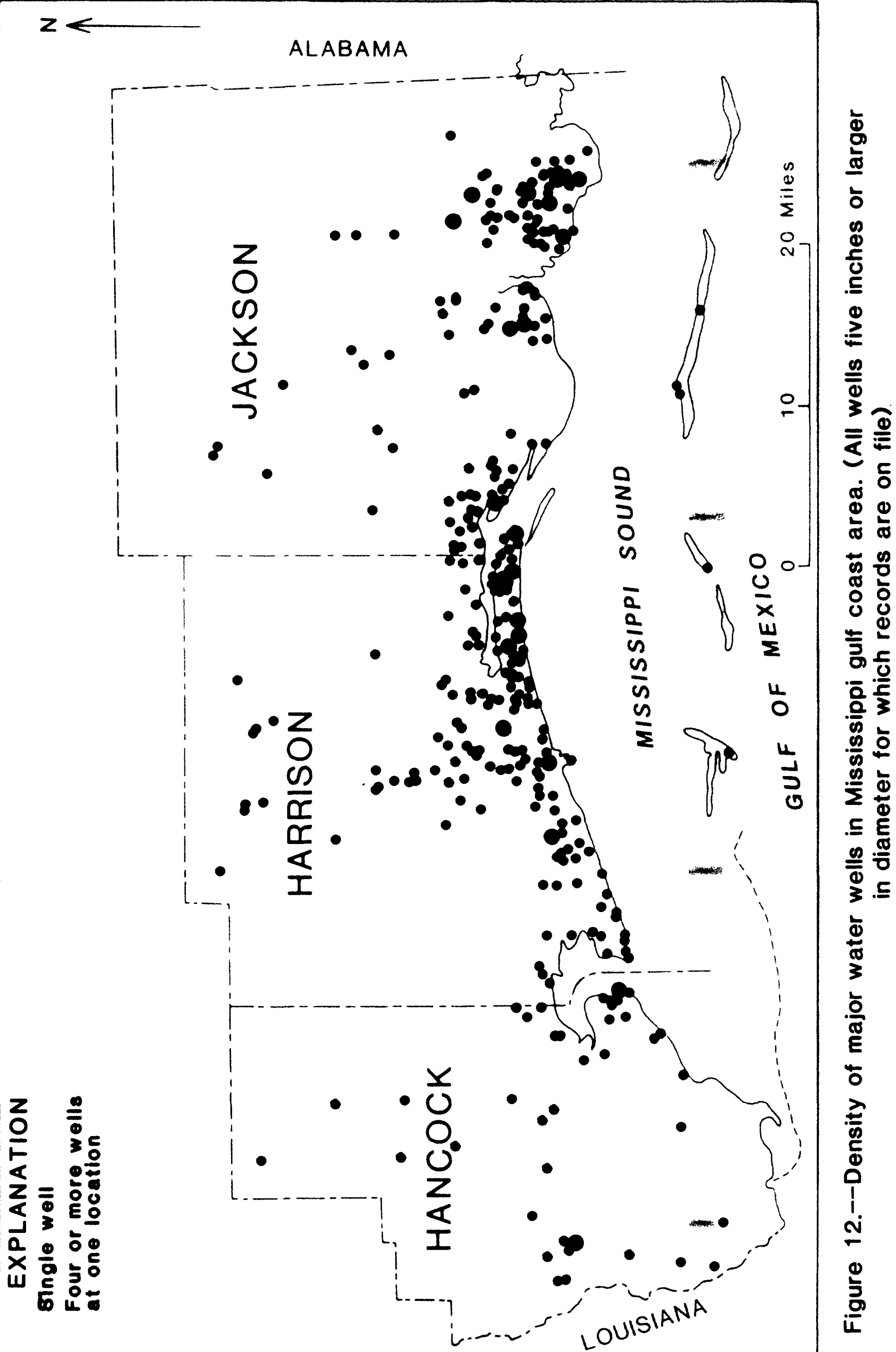




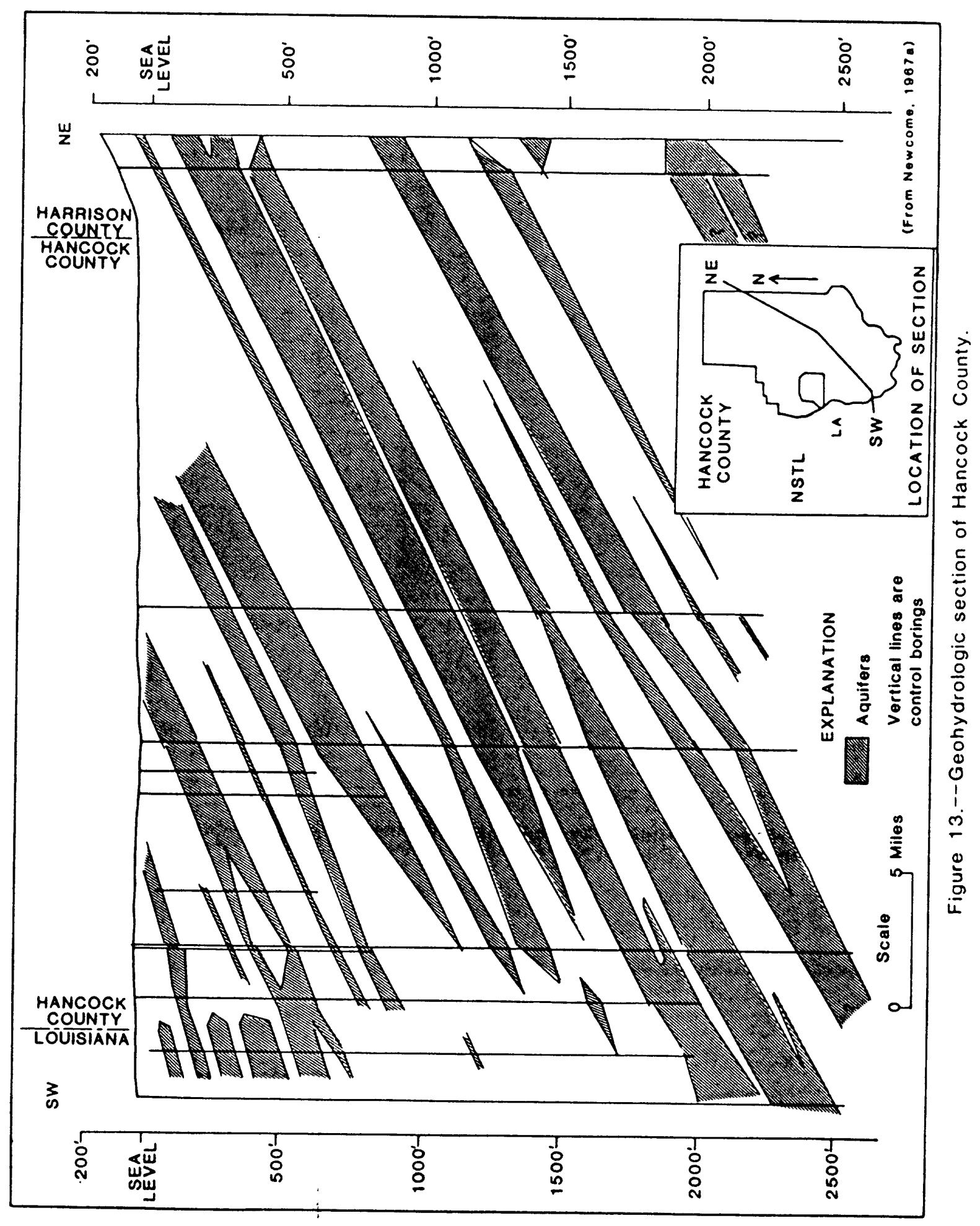




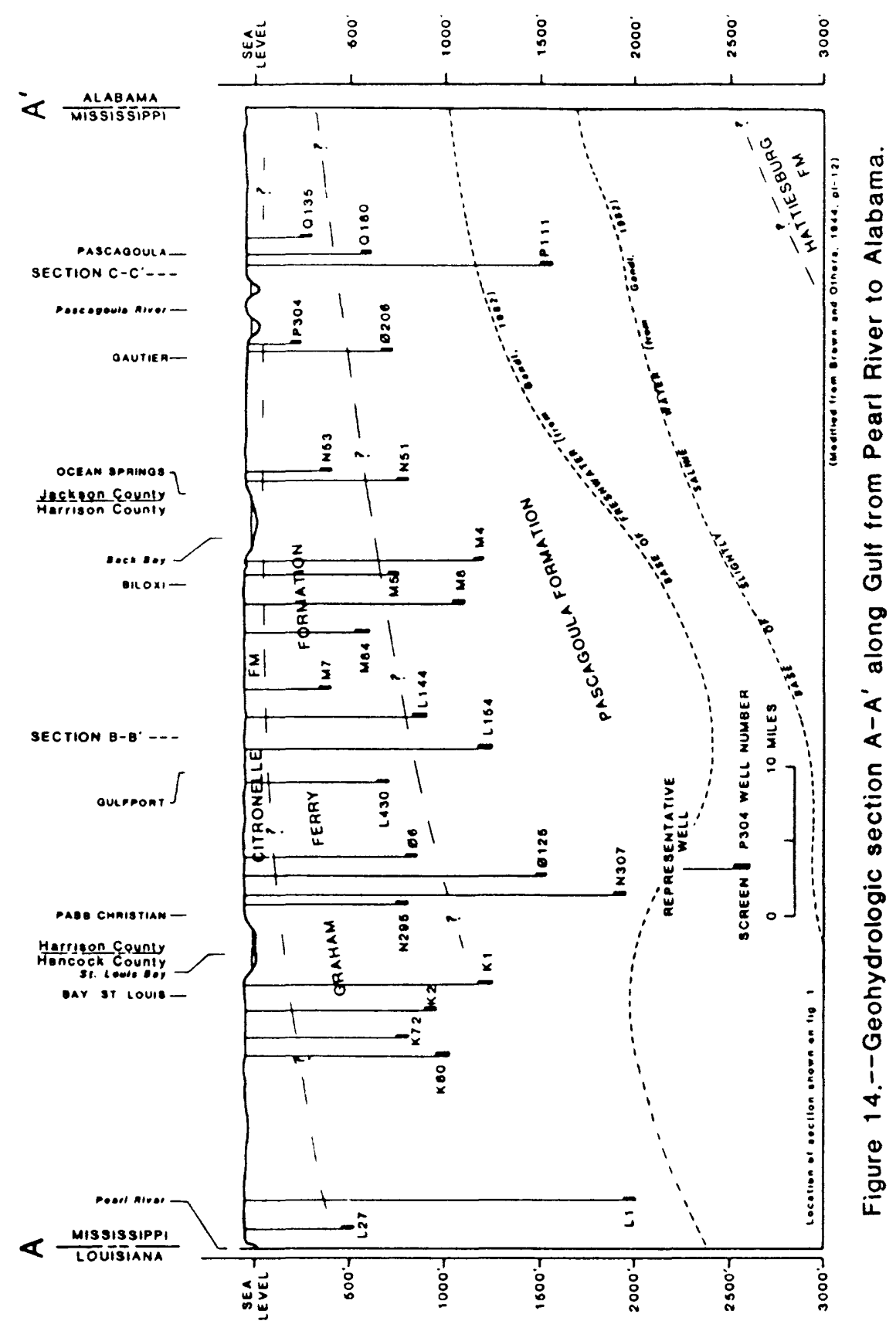




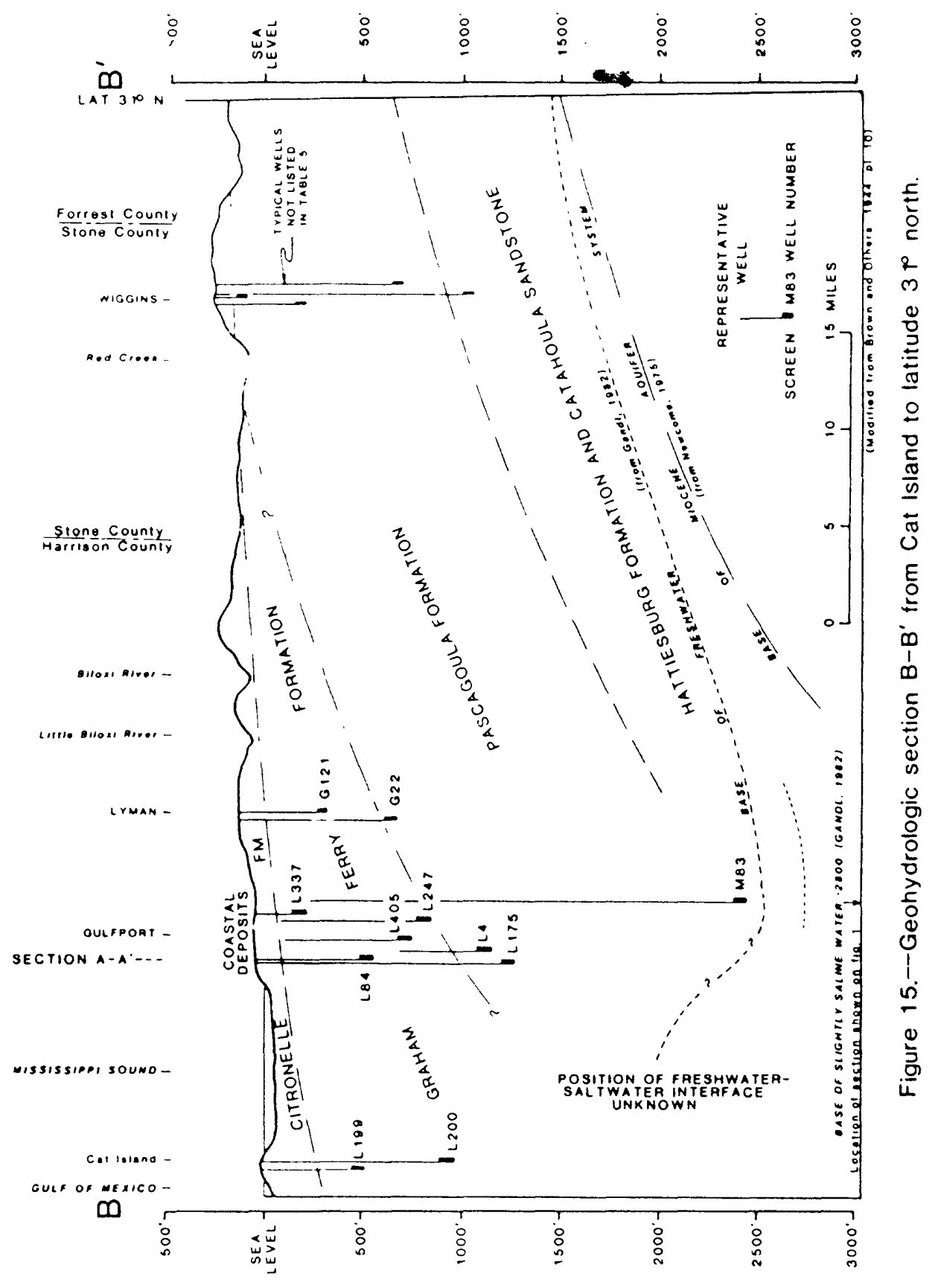




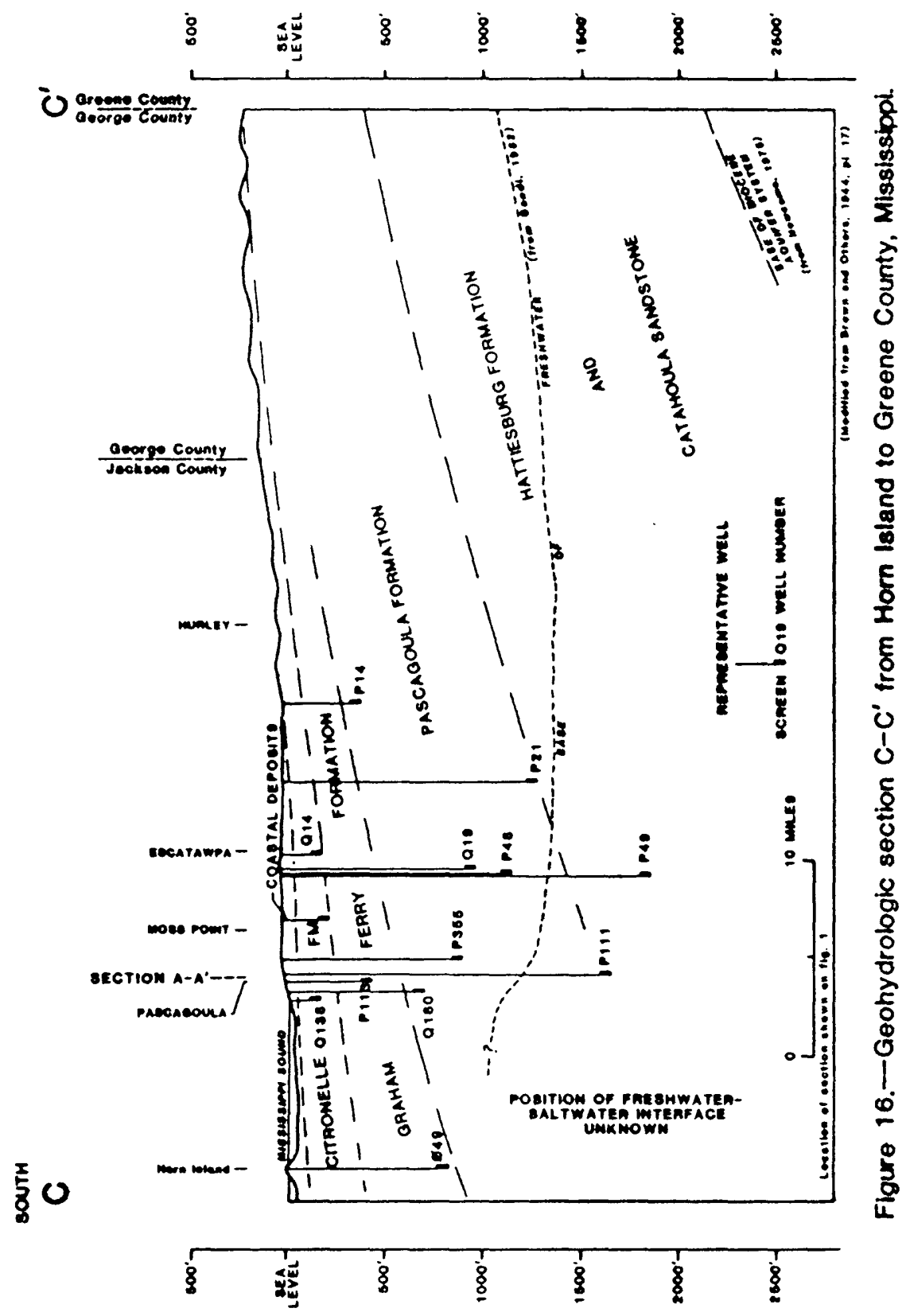


Aquifers in the gulf coastal counties include the Miocene aquifer system (Newcome, 1975), the Citronelle aquifer (Boswell, 1979) and aquifers in younger alluvial and coastal deposits (table 1). According to Newcome (1975),

\begin{abstract}
"The Miocene sequence in southern Mississippi has been subdivided by some workers into the Pascagoula Formation, Hattiesburg Formation, and Catahoula Sandstone--from youngest to oldest--but these divisions cannot be reliably identified or traced in the subsurface. Likewise, a 400- to 900-foot thick unit at the top in the coastal counties has been identified as pliocene in age on the basis of fossil evidence and assigned the name Graham Ferry Formation. Again, the unit cannot be distinquished from the next lower formation by lithological, geophysicalsor hydrological means. Consequently, all the material between the Citronelle Formation, a blanket deposit of Pliocene age, and the limy Vicksburg Group of 01 igocene age is herein considered to compose the Miocene aquifer system."
\end{abstract}

Although the formational subdivisions of the Miocene Series are not accepted by all authors, the units as delineated by Brown and others (1944, plates 7, 10, and 12) are useful in defining water-bearing zones and in general correlations of aquifers in the coastal area.

The Citronelle Formation, a blanket-type deposit that partly overlies the recharge areas of all older coastal aquifers (fit. 2 ), is relatively flat-lying, sloping southward at a rate of less than 10 feet per mile (Boswell, 1979). Erosion has dissected the formation, and valleys a few miles north of the coast are incised into the underlying formations. According to Brown (1944) near the coast the citronelle questionably has continuity into the subsurface and is covered by younger deposits (fig. 2). Minor aquifers occur in alluvial deposits in the larger valleys and in the younger coastal deposits that overlie the Citronelle Formation.

The source of water in all coastal aquifers is precipitation on the outcrop (recharge) areas. The water initially moves downward until it reaches the water table or a layer of impermeable material. Water then moves laterally until it discharges into streams or moves generally southward down the dip beneath confining beds toward areas of artificial discharge (pumping wells) or natural discharge (upward leakage to shallow sediments or to the sea beyond the barrier islands).

Identification of recharge areas is complex, because of the blankettype deposits of the citronelle Formation that overlie the older aquifers (fig. 17). Much of the precipitation is first absorbed by and stored in the highly permeable Citronelle and subsequently moves into the underlying aquifers or discharges into streams. The rate and volume of recharge is controlled by the hydraulic head in the aquifers and the thickness, character, and degree of interconnection of beds. Inland recharge areas are considerably above sea level. The regional hydraulic 


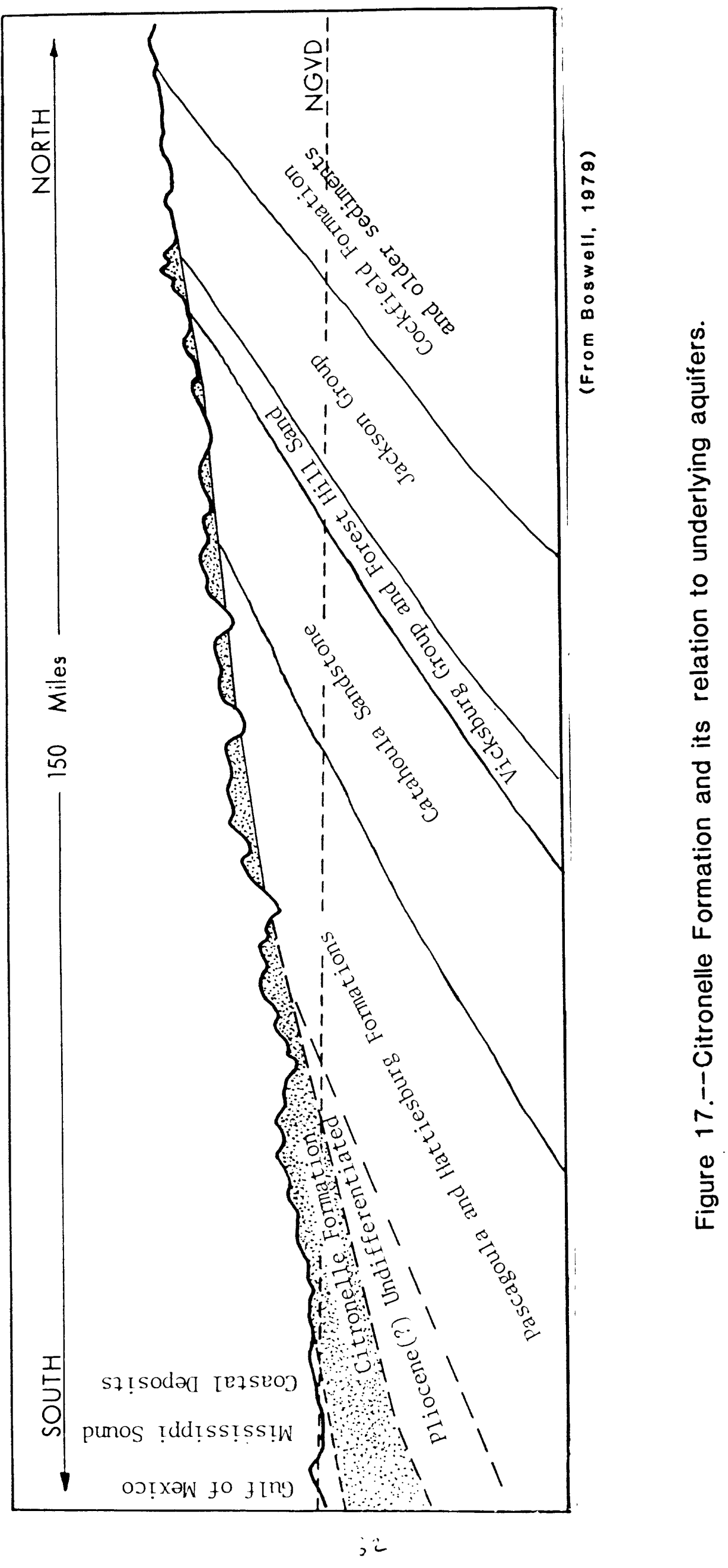


gradient is low, less than 5 feet per mile, and the gulfward slope is interrupted by cones of depression where large volumes of water are pumped (fig. 18). Generally, the recharge areas lie to the northnortheast (fig. 2). Newcome and others (1968) concluded that the shallowest aquifers that are commonly tapped by large capacity wells along the coast are "replenished in an east-west band that crosses the south end of Stone County" and that the aquifers that occur at intermediate depths along the coast are recharged in an east-west band at the latitude of Wiggins. The deepest freshwater aquifer receive recharge in an area north of the latitude of Wiggins. The deepest aquifers that contain freshwater just north of the study area in Pearl River, Stone, and George Counties contain saline water in the coastal counties (Gand), 1982).

Wells on the barrier islands confirm that freshwater in some of the coastal aquifers occurs at least as far south as the islands, and the relatively low dissolved-solids content of water from these wells indicates that the freshwater-saltwater interface in the shallow to moderately deep zones is probably some miles to the south of the islands. The deepest water wells on the island do not exceed 1,200 feet and thus the Gulfward extent of freshwater in the deeper coastline aquifers is unknown.

The source of most fresh ground water used in the Gulf Coast area is the upper part of the Miocene aquifer system in strata considered to belong to the Graham Ferry Formation as defined by. Brown (1944, plate 12). In the Pascagoula area, the entire freshwater section of the Miocene aquifer system is developed, but from Gulfport westward only a few wells penetrate the lower part of the system (figs. 14, 15, and 16).

Recent studies (Gandl, 1982) show that the base of the freshwater zone in the system increases in depth from about 1,200 feet east of Pascagoula to more than 2,400 feet in the Gulfport area and to about 2,000 feet at the mouth of the Pearl River and that the deepest freshwater extends slightly below 3,000 feet (fig. 19) in the western part of Hancock County. The base of the slightly saline zone increases from about 1,800 feet east of Pascagoula to about 3,200 feet in western Hancock County. The depth to the base of the moderately saline zone increases from 2,000 feet to over 3,600 feet in the same area (fig. 19).

Most large wells in the coastal counties are made at depths ranging from about 400 to about 1,000 feet and only a few wells exceed 1,200 feet in depth (table 5). Among the exceptions are a well 1,900 feet deep in Port Bienville Industrial Park (Hancock County) and a 2,400-foot well drilled recently at Gulfport. Wells commonly produce 500 to $1,000 \mathrm{gal} / \mathrm{min}$. Very high pumping rates are feasible in some places; for example, the highest measured pumping rate was $5,865 \mathrm{gal} / \mathrm{min}$ from a 672-foot well at National Space Technology Laboratories (Newcome, 1967 a, p. 12). 


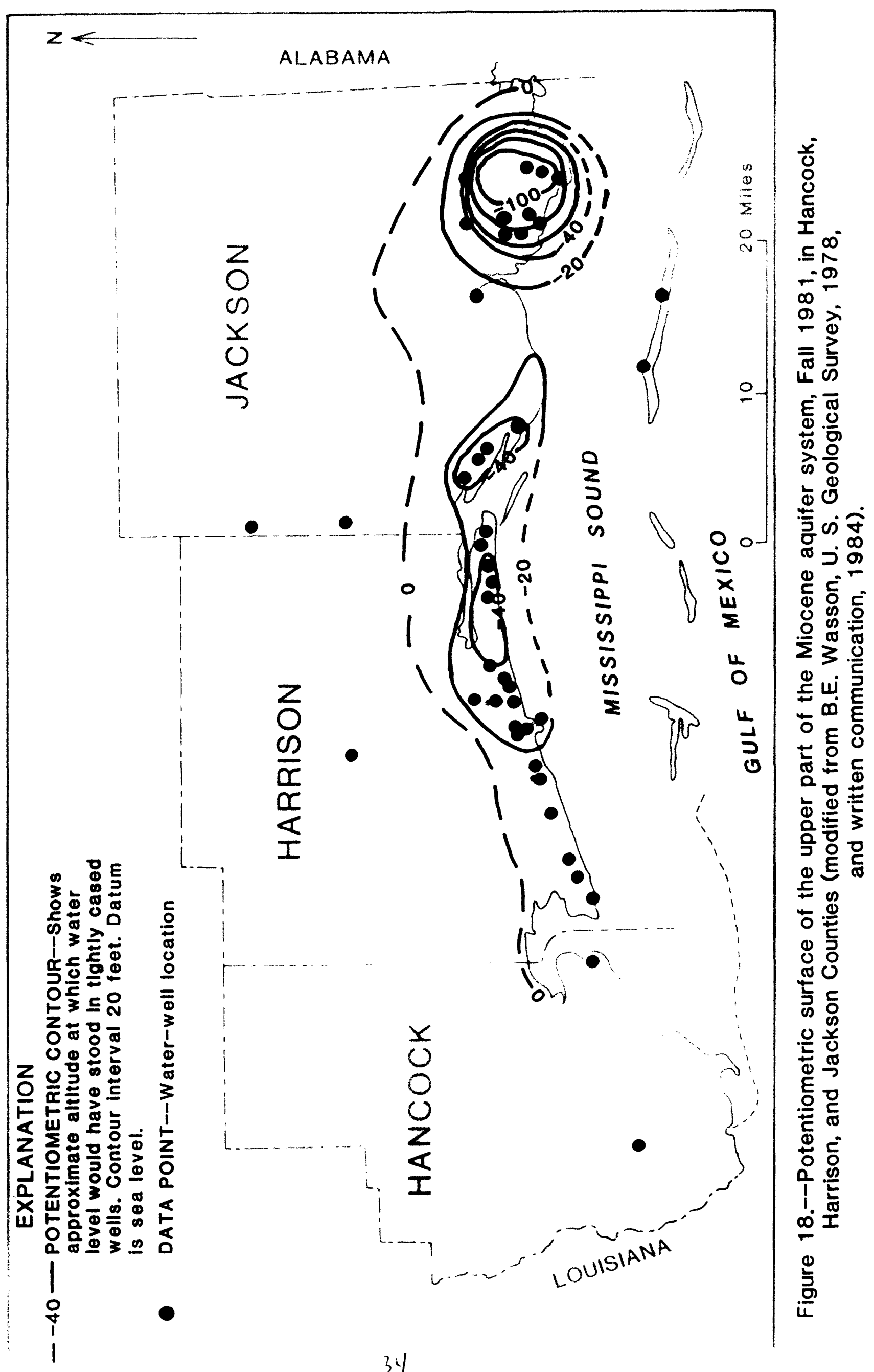




\section{EXPLANATION}

-2400 - Contour showing altitude of the base of the specified zone. Contour interval is 200 feet. Datum is sea level.

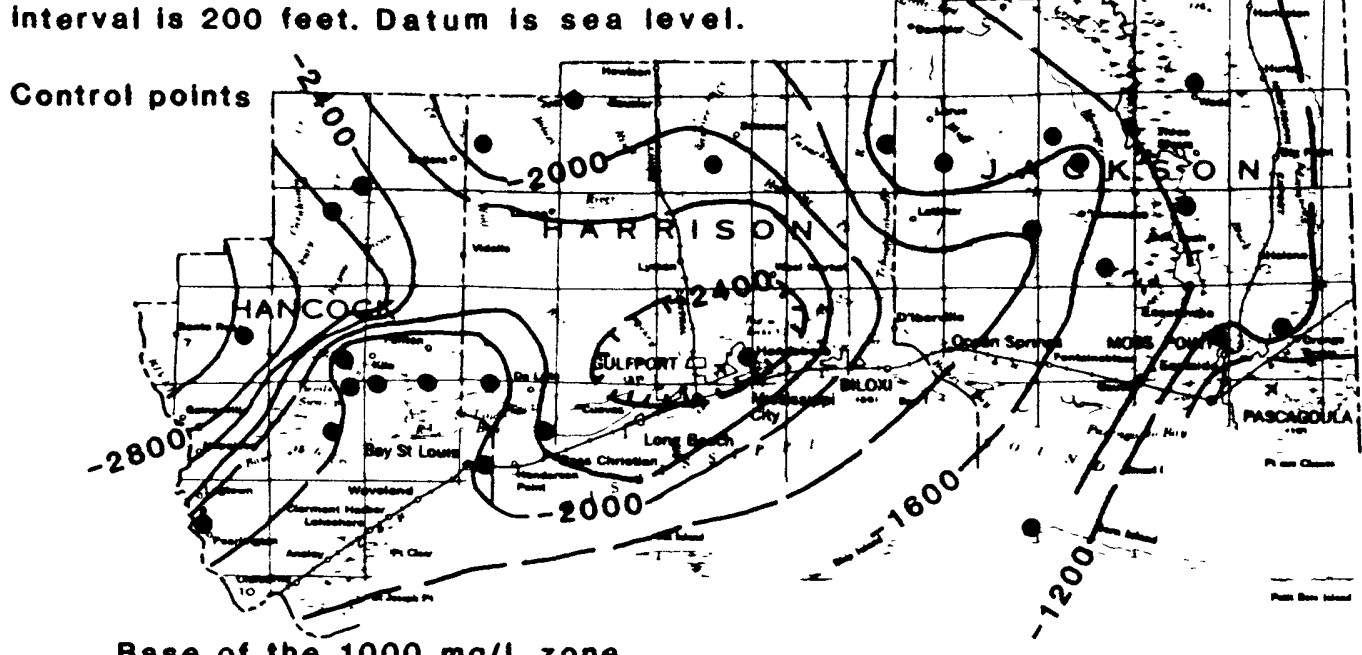

Base of the $1000 \mathrm{mg} / \mathrm{L}$ zone (freshwaten)

(From Gandl, 1982, pl. 1)

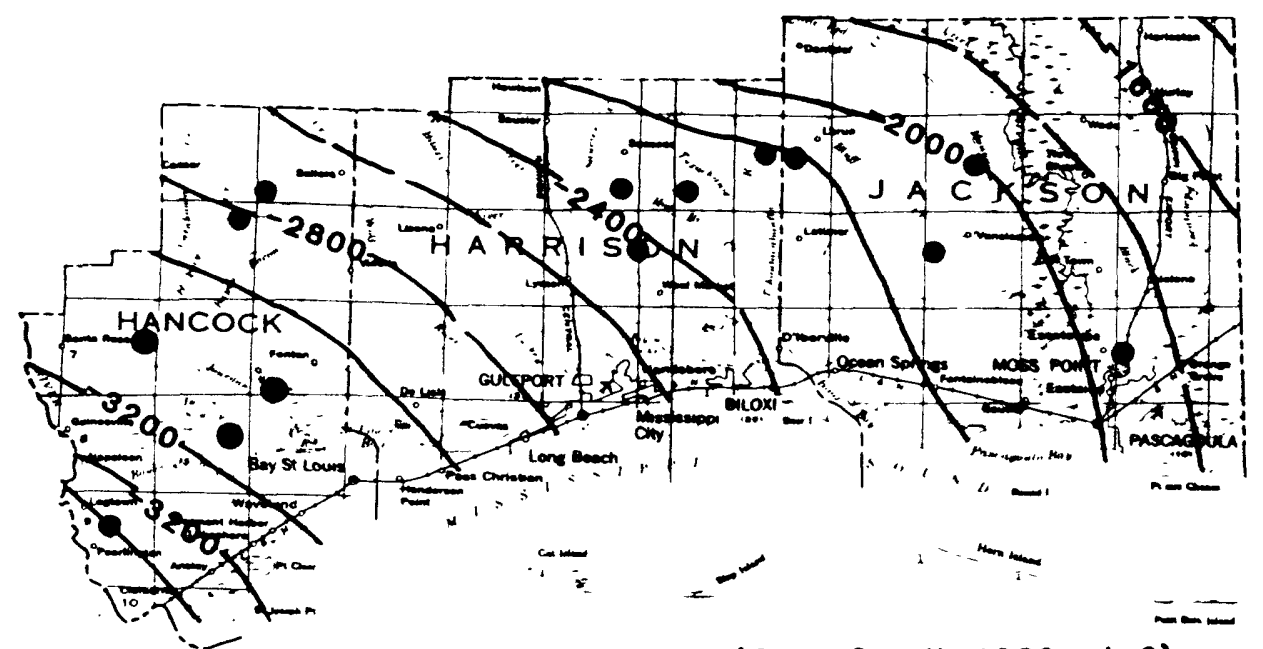

Base of the $3000 \mathrm{mg} / \mathrm{L}$ zone

(From Gandl, 1882, pl. 2) (slightly saline)

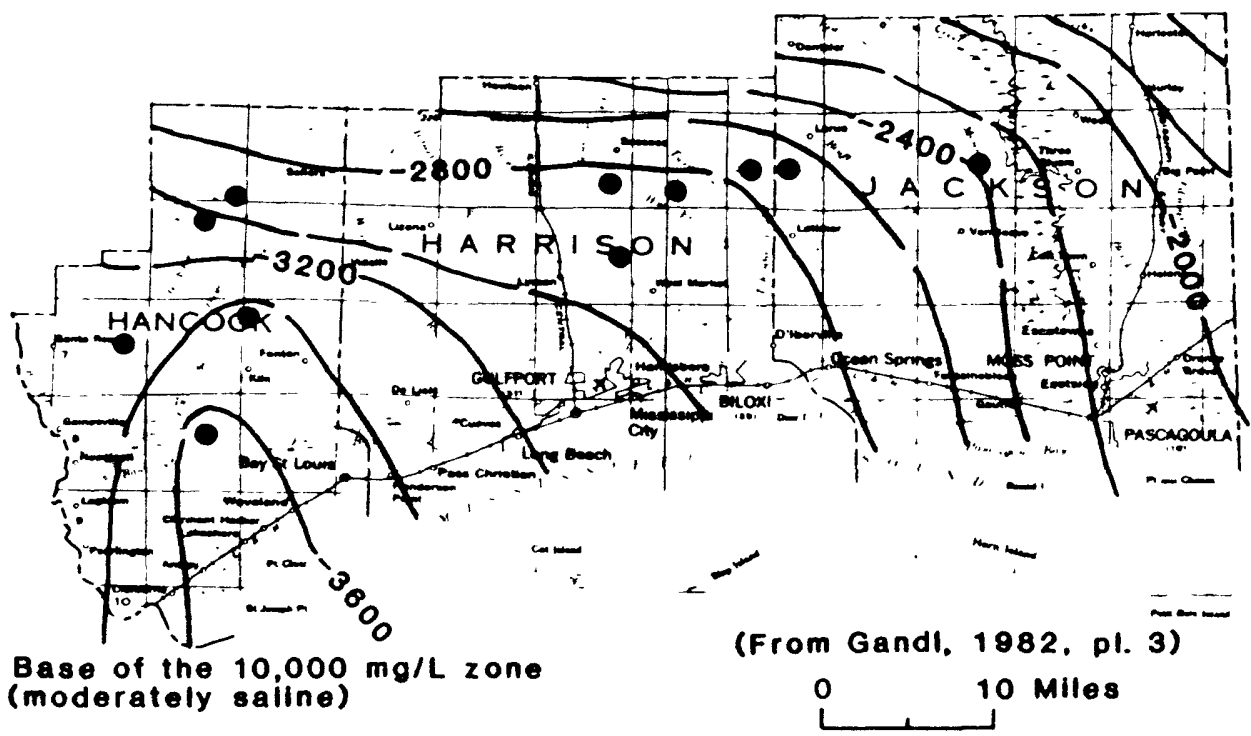

Figure 19.--Configuration of the bases of the moderately saline, slightly saline, and freshwater zones in the coastal counties, Mississippi (modified from Gandl, 1982). 


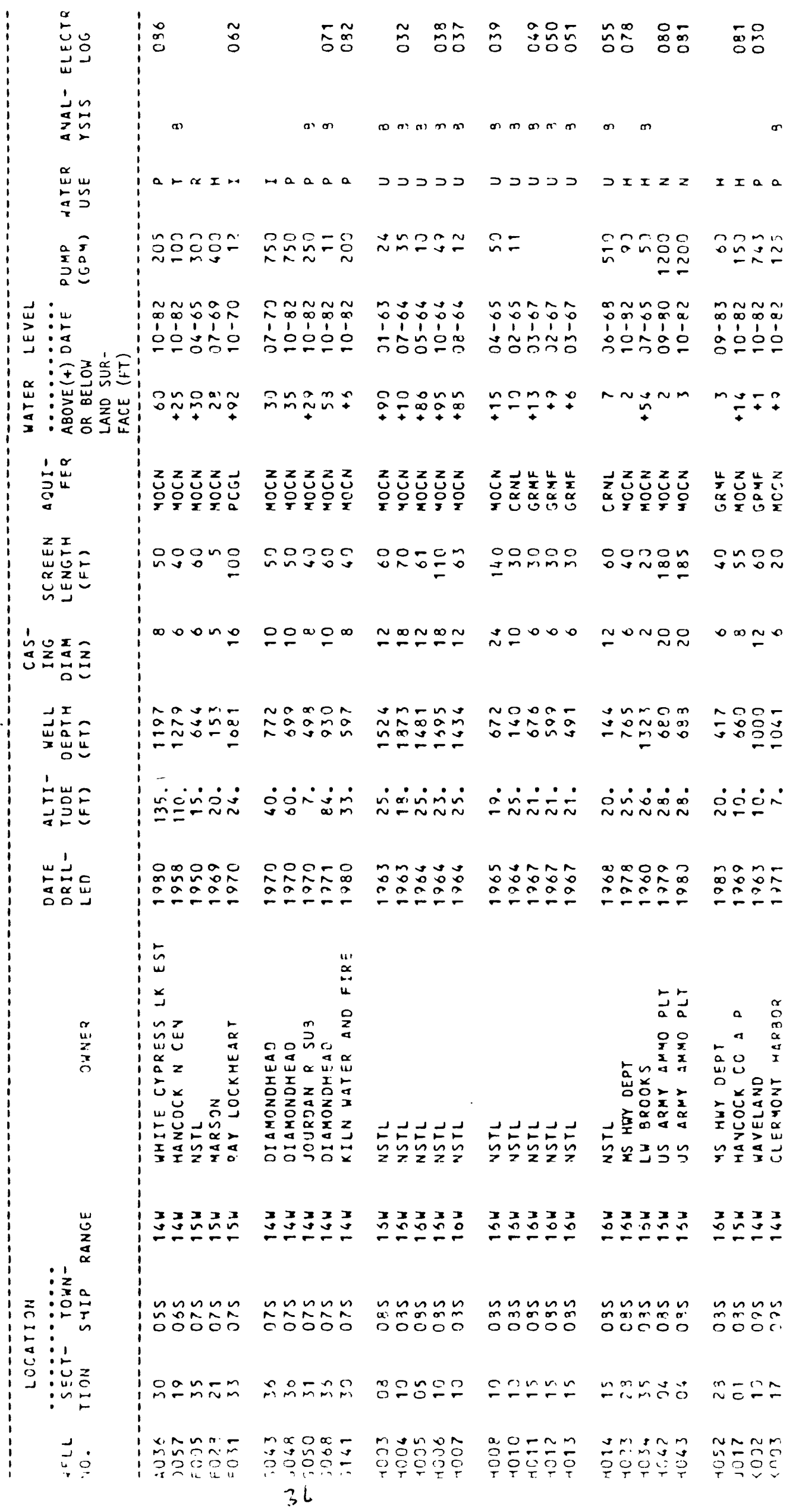


宅总等石

设

$\sim \sim n$ mกำ

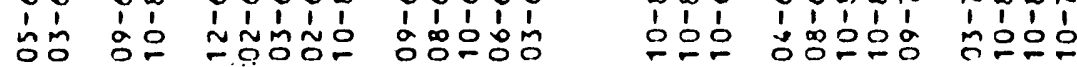
仙要要

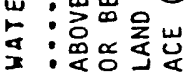
- $\tilde{0}$ น

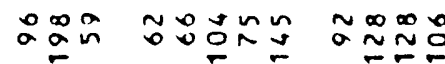

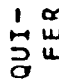

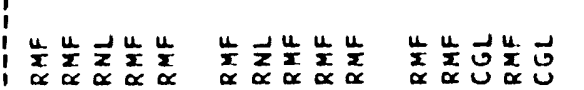

zI $I$

崖岂出

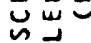

$x_{x=1}$

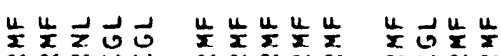

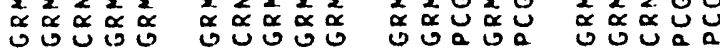

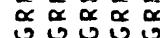

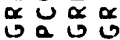

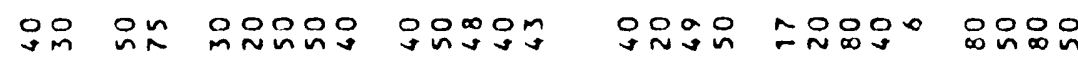

吉去 뜸는

i.

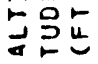

崖光。

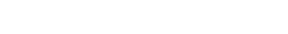

NOOON DOONO - ONOM DOOMN MOONO NNOD

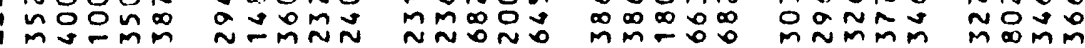

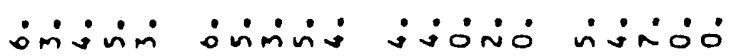

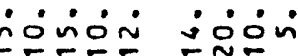

ํํㅇํำ

ล으응

ana

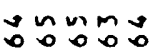

a 000

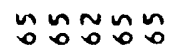

ลัํํㅇำ

MOOOON

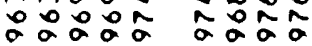
(1)

언워

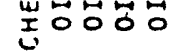

웅웅우

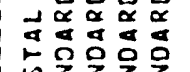

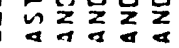

跨去台台

こ

$n$
5
5
0
0
0
0
0

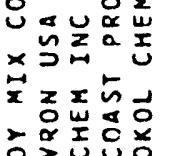

出吕过界

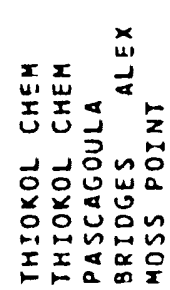

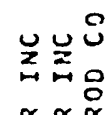

吕品品品

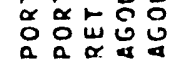

م a

$x \times z \backsim n$

ara

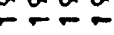

$\left\{\begin{array}{l}3 \\ z \\ z\end{array}\right.$

:

ㅆ

I

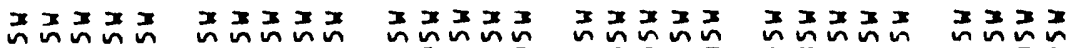

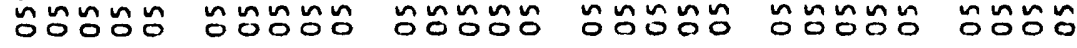

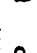

$\pm:$

$\Delta: 1$

ㅇ: : us

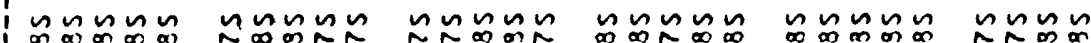

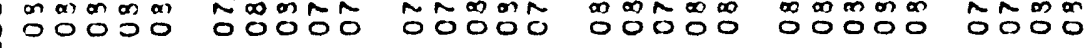

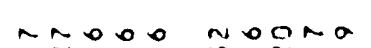

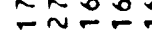

MOำ

$\sim \sim \sim ⿻ 上$

$-\sim \sim 00$

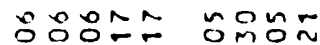

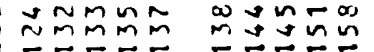

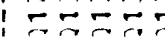

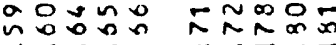

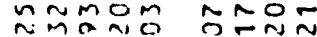
37

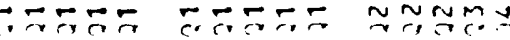




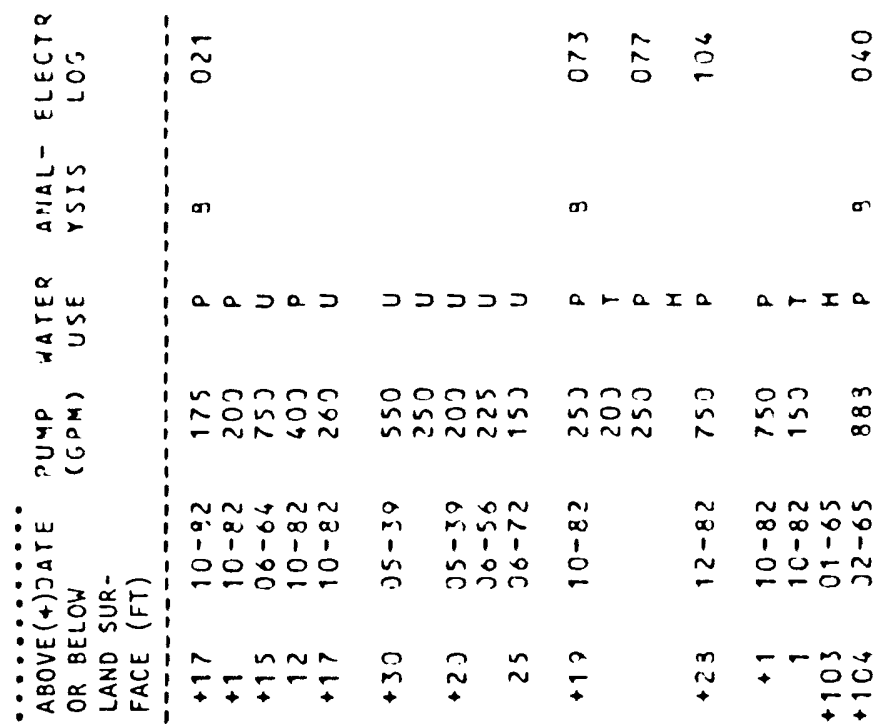

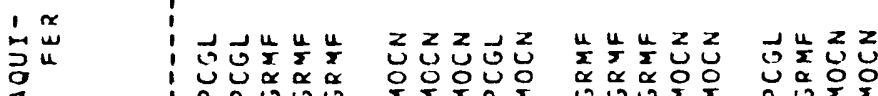

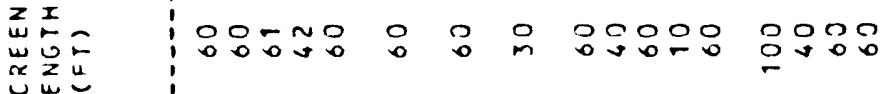
出

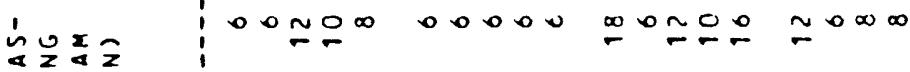

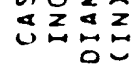

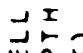
$\geq$ w

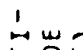
농 !

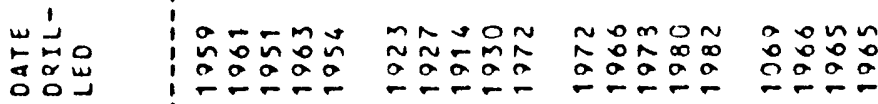

: z:

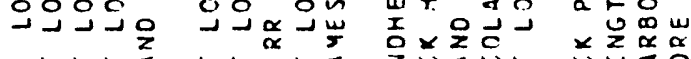

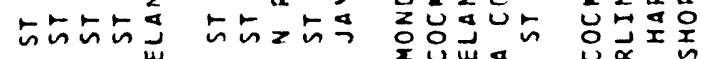

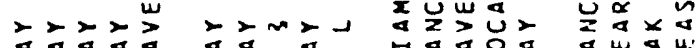

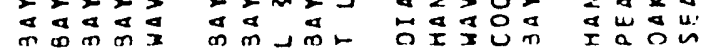

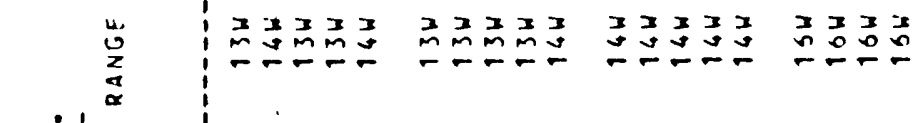
$: \frac{1}{2}$

z:ํำ

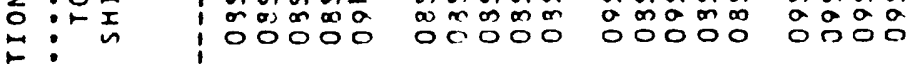

ㄴ:

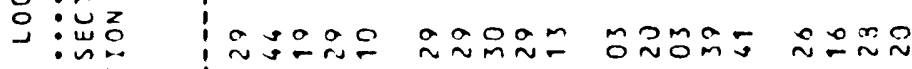

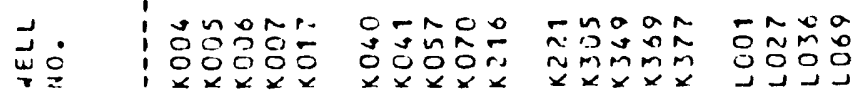


$x$ I

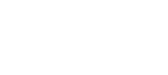
000 $a-a>$

$$
\text { 잉음 }
$$

mon

음ㅇㅇㅇ응

$8=80$

๓๐์

moñ

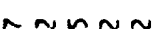

$0 \sim N N$

$m$

$m m \infty n$

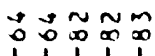

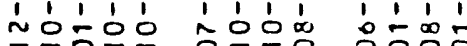

응든

\section{品}

$z_{w}^{x}$

$\underset{\alpha}{*}$

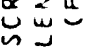

ins:

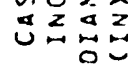

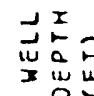

1

뜸를

a $m \sim m a$

$m m i=r$

$\tilde{n} \sim \sim \tilde{m}$

$0 m+2$

nm nog

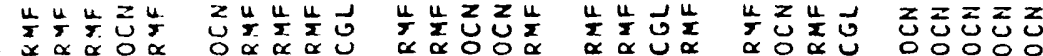

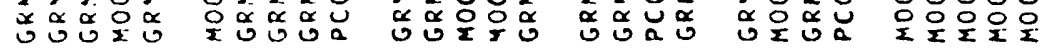

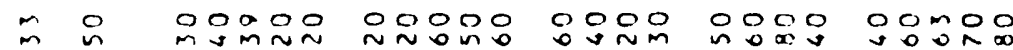
$n \sim n \infty 0$

$\infty \infty \infty \infty$

$\operatorname{can}$

$\simeq \simeq \infty$

$\simeq \sim ㅇ$

$\sim \sim \infty \sim \infty$

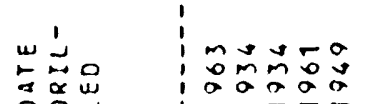

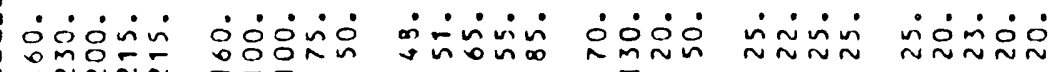

○。 $-\frac{2-2}{2}$

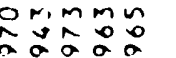

$m \underset{m}{m} \tilde{m}=0$

은ñ

ỡน

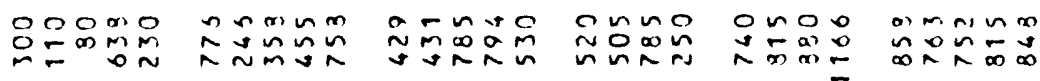

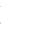

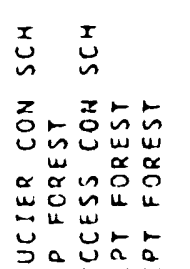

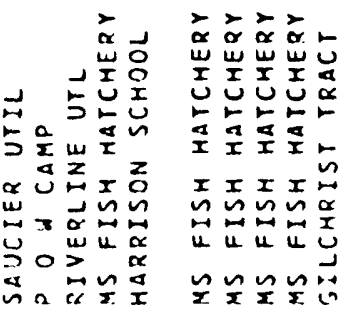

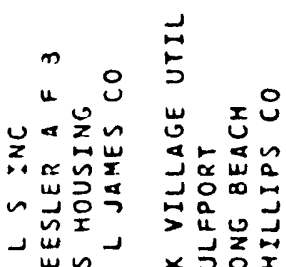

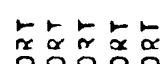

응ㅇㅇㅇㅇㅇㅇ

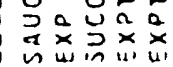

n)

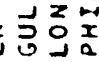

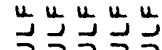

\begin{tabular}{l|l}
$w$ & $x \geq 3 \geq$ \\
2 & $5=5$
\end{tabular}

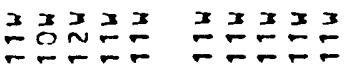

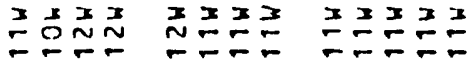

01

$: \geq$

亏: : :

客

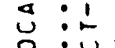

o: : u

エேニニニ

in

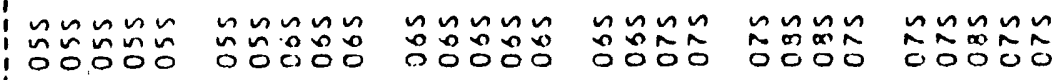

○ั000

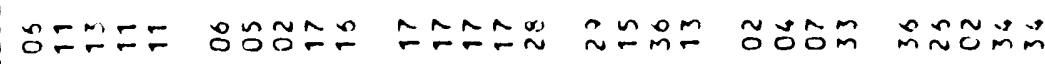

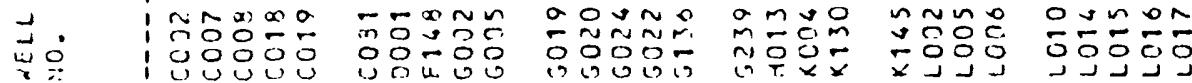




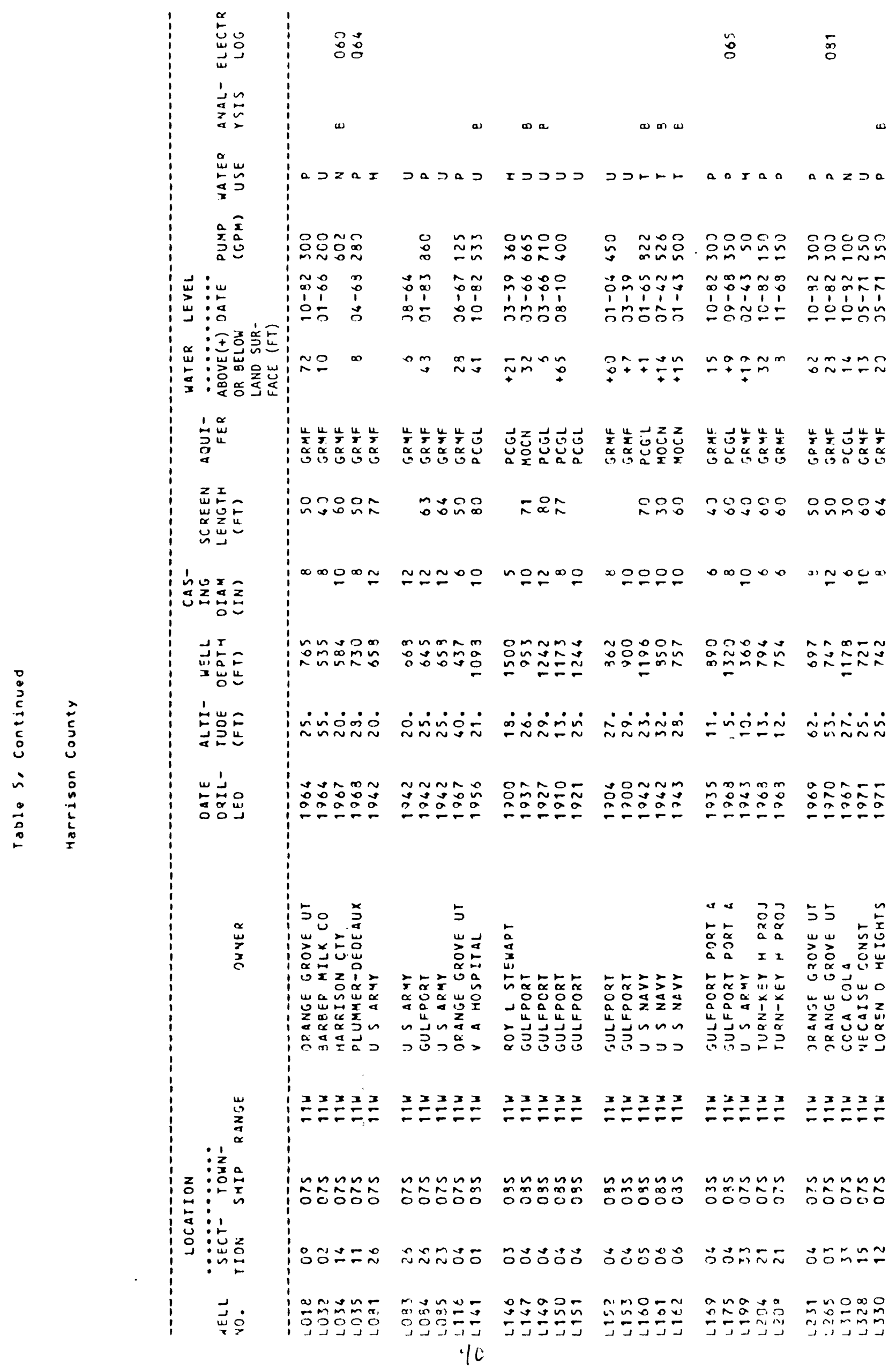




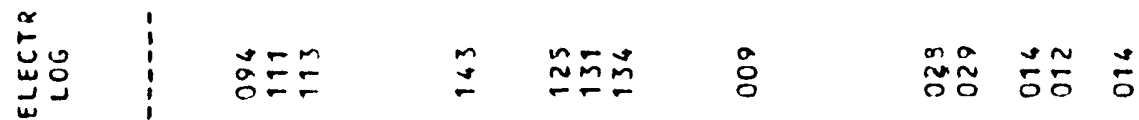
范药 $\stackrel{8}{\stackrel{0}{a}}$

âd

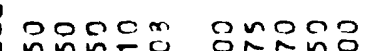

은으요

$\because 00$

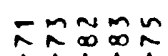

$\vec{w}:$ w

出:

:王辛穿

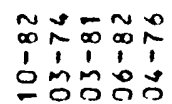

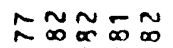

în.

óóá

ํํㅇำ

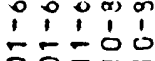

$\approx$

$\approx \approx \infty$ 品的吕

谂㟧

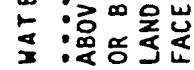

ํํㅁำ

omann

mmmos

nูก๊ก

maño

10 क क ⿻ 丨

añ

0000n 1111

a

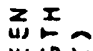

㟧起出

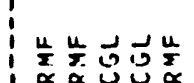

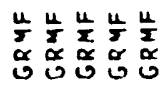

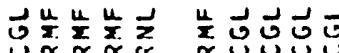

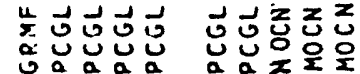

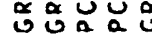

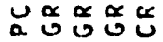

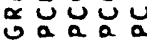

نئ

in

iñ 0

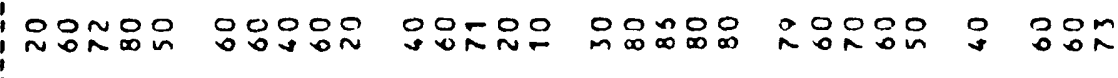
Uேีே

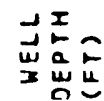

is

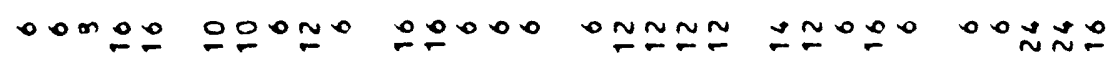

$\pm ฏ$

幽雚。

인

im $m$ on

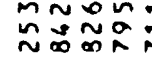

anoon

응요

กัน

i isin

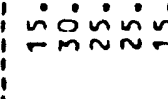

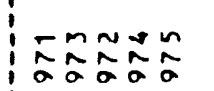

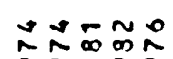

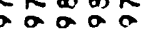

$\hat{\sim} \leqslant \hat{\infty} \infty \infty$

ลิล์ิ์

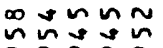

ถ๐ํํำ

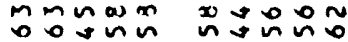

:

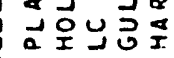

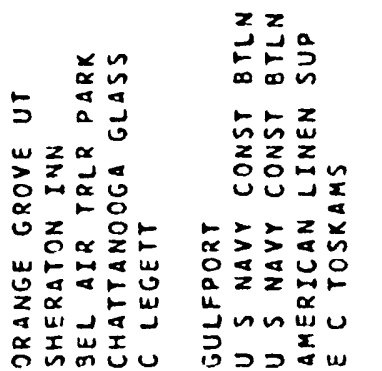

2
$\vdots$
0
0

ă

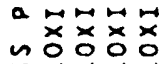

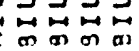

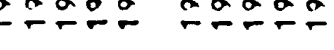

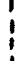

:

岕

포픈

$\geq \geq \geq \geq \geq$

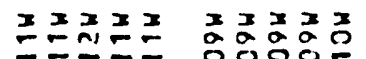

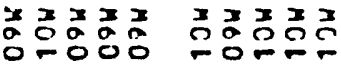

중 :

:

实:

满

岃 1

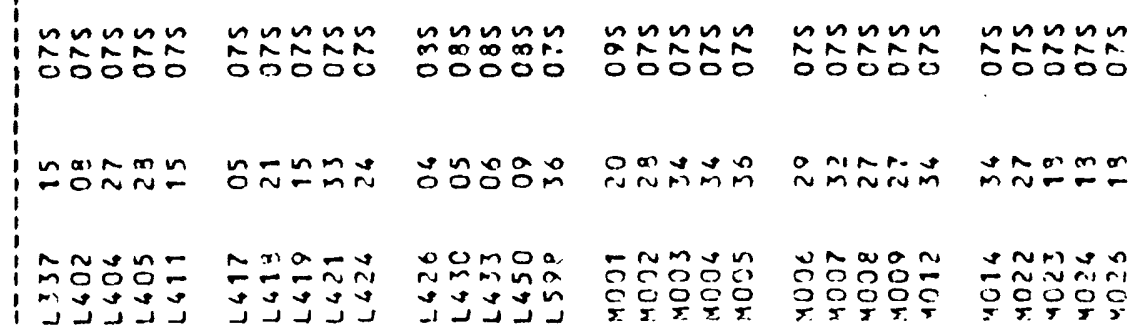




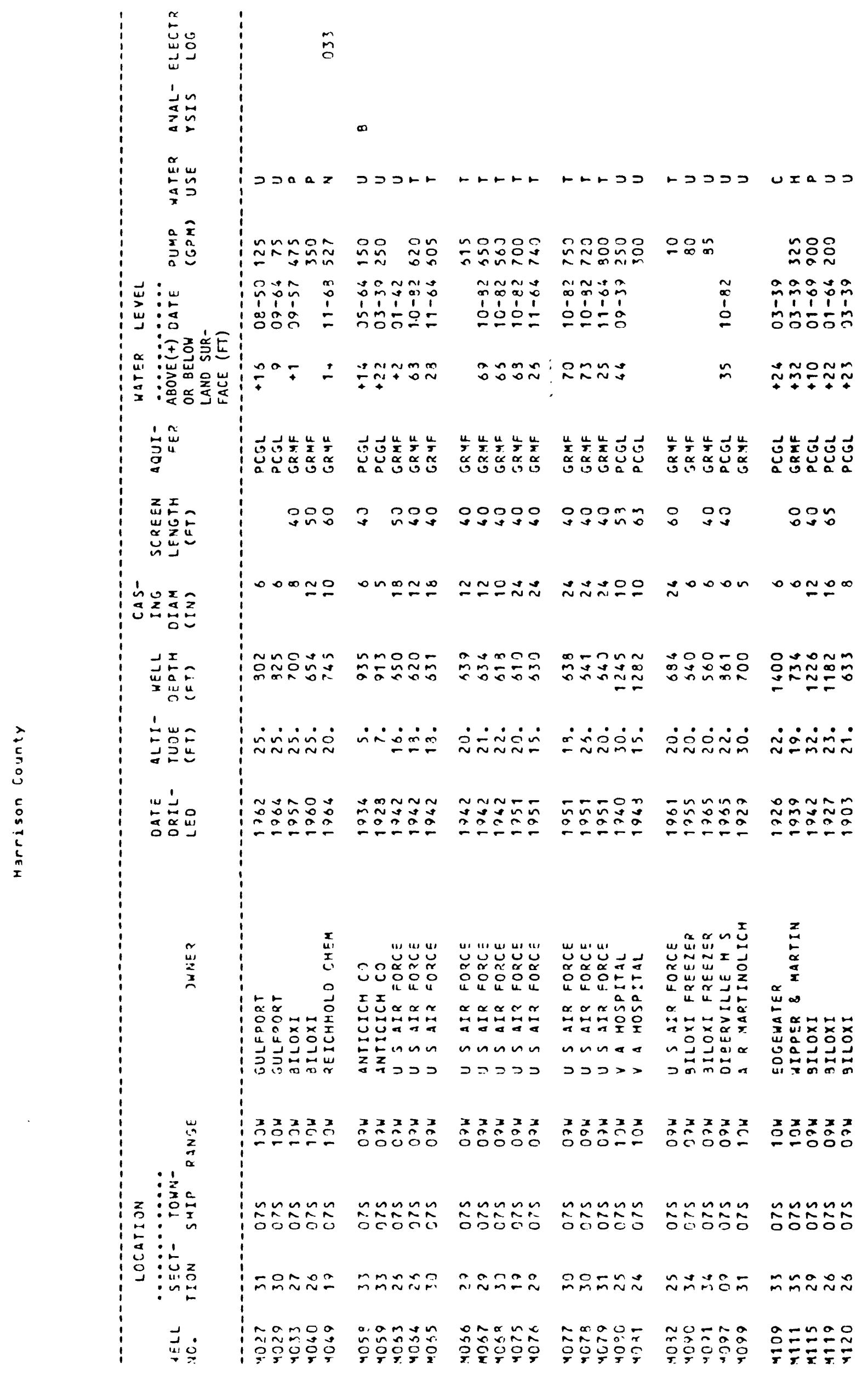




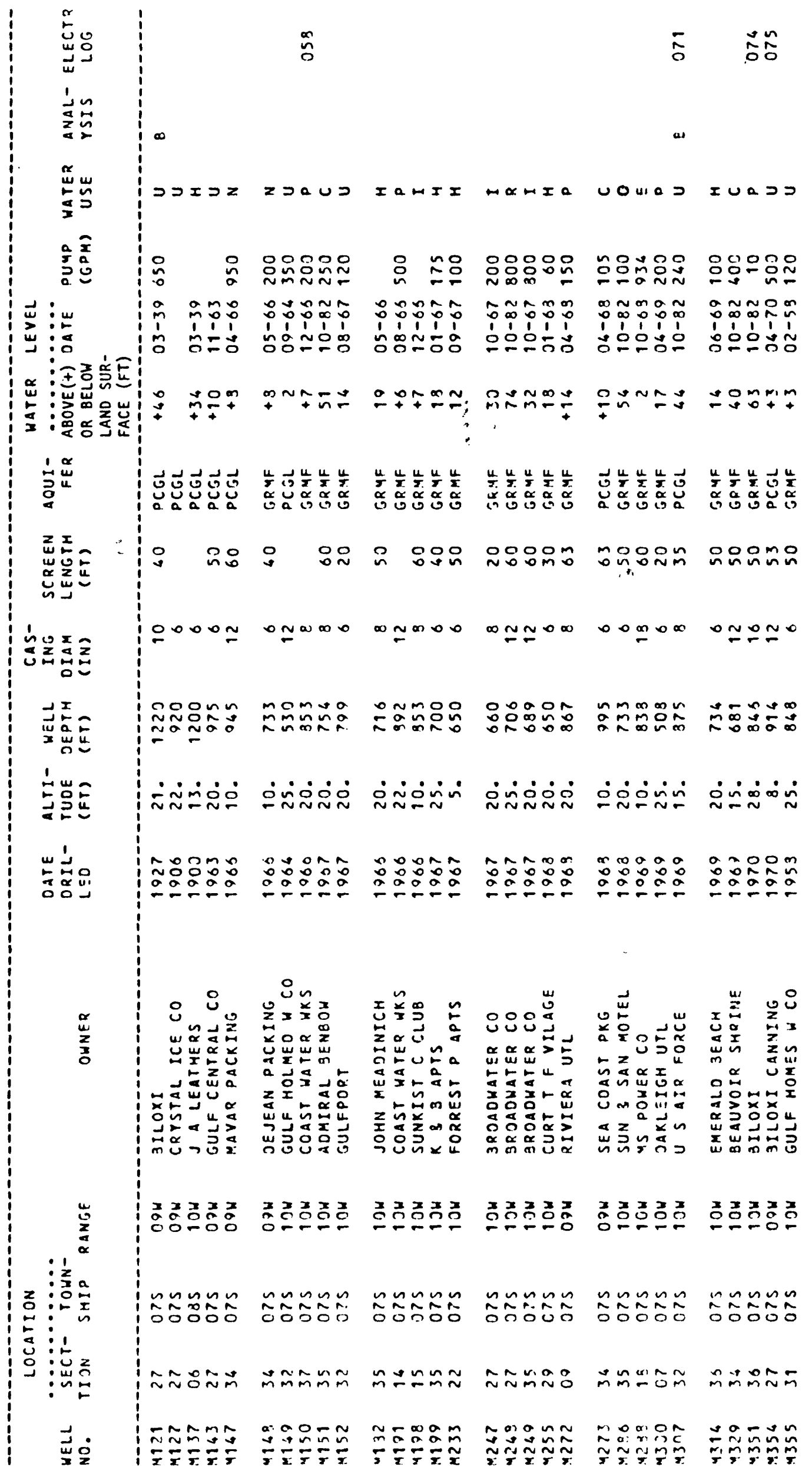


JวaลU IIIU $u \supset u \geq z$

$z \supset \supset a I$

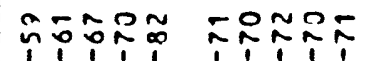

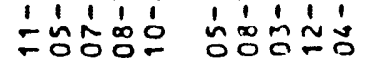

mố:

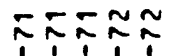

- $\approx$ N

*ก๊

:

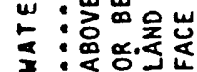

实出

$\mp \mp+\tilde{m}$

ำกำ

ㄷำำ

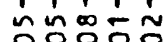

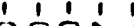

ㅇํㅇㄷㅡ

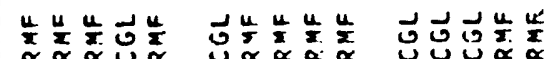

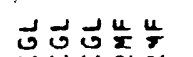

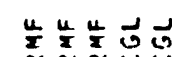

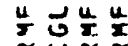

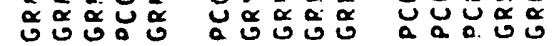

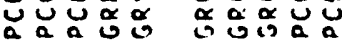

웡

出

幽苾台 은옹은

웅옹ㅇㅇㅇ

용ㅇㅇ

0

000

은으

은요옹

is

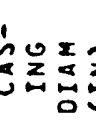

J5 Iu

는

음는

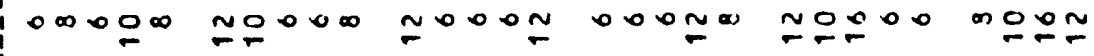

$\stackrel{5}{\leftarrow}$

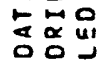

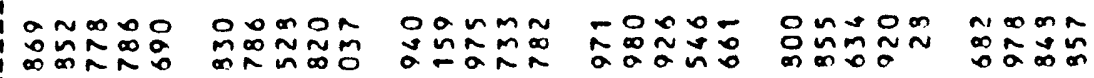

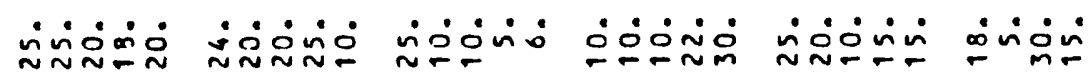

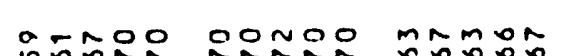

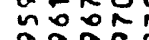

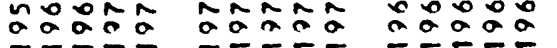

ำกัล

tomm

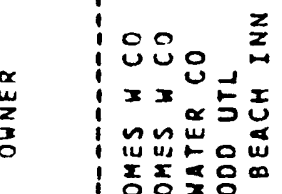 \\ 일

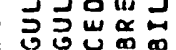

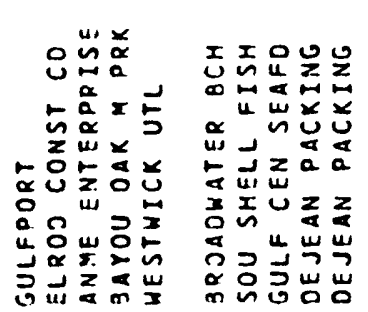

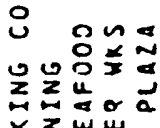

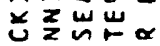

a $u_{\alpha} \propto \omega$

이물

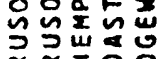

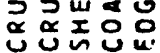

-

\begin{tabular}{|c|c|c|c|c|c|c|}
\hline 㟧 & 콤ㅇㅁㅇㅁㅇ & 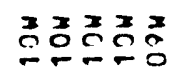 & 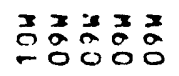 & 超 & 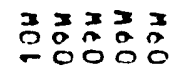 & 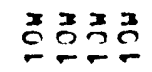 \\
\hline & 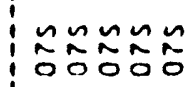 & 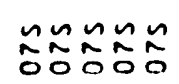 & 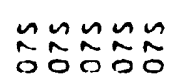 & 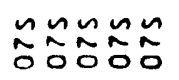 & 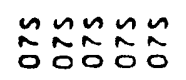 & 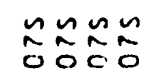 \\
\hline & $\bar{m}=\simeq \simeq m$ & $\tilde{m} \approx a \tilde{a} \tilde{\sim}$ & $\tilde{\sim} \sim \dot{\sim} \dot{m} \dot{m} \dot{m}$ & 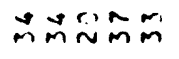 & $\stackrel{\sim}{\sim} \approx \tilde{\sim} \sim$ & $M \tilde{M} \tilde{\sim}$ \\
\hline & 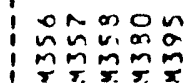 & 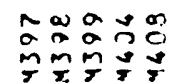 & 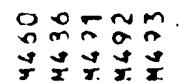 & 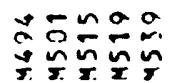 & 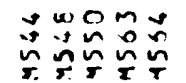 & \\
\hline
\end{tabular}

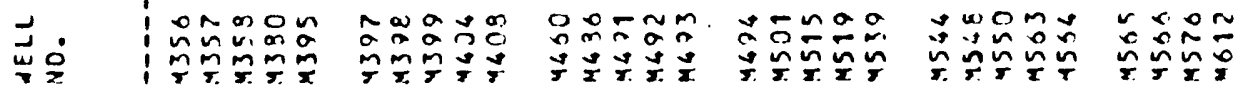




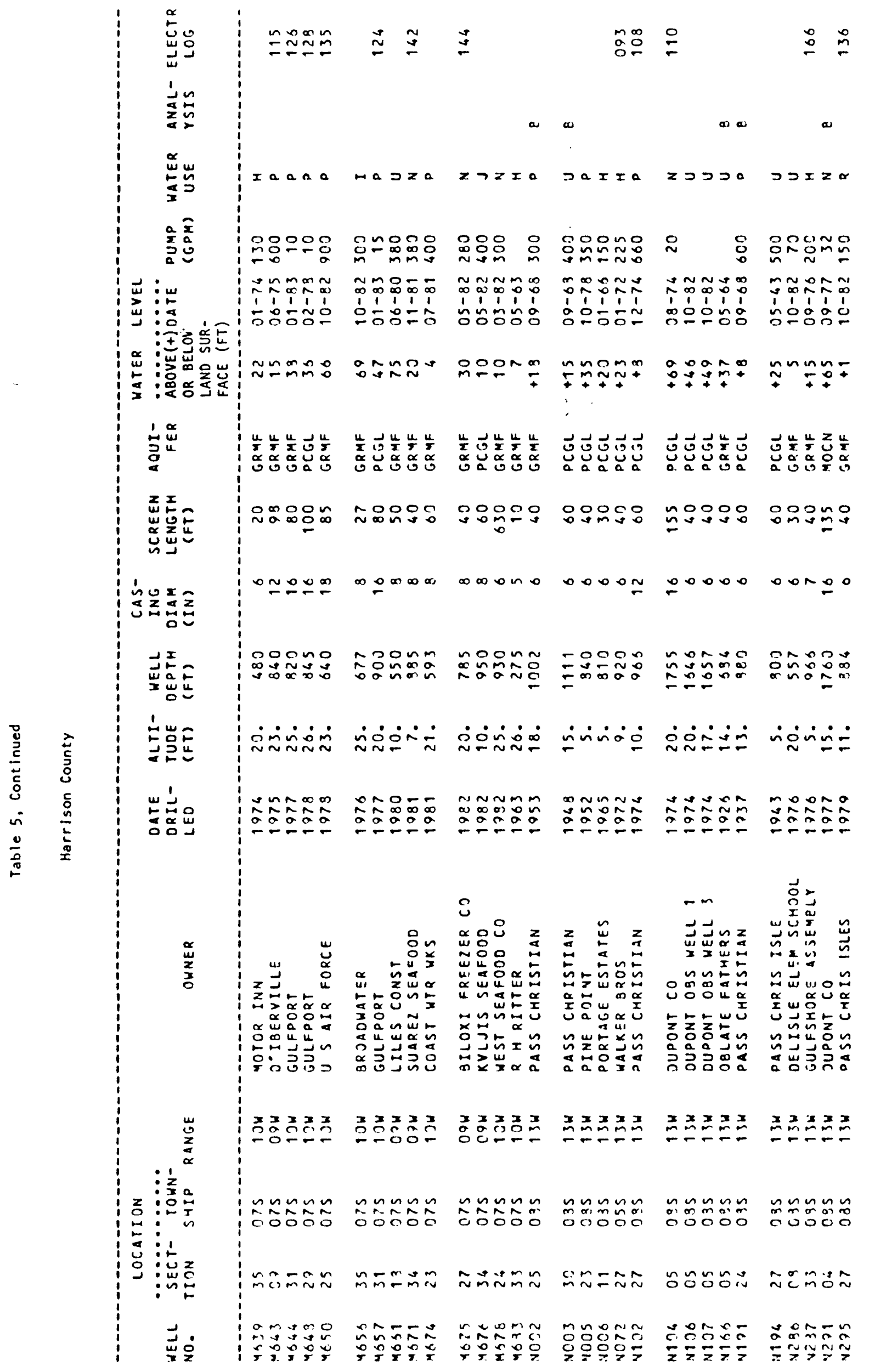




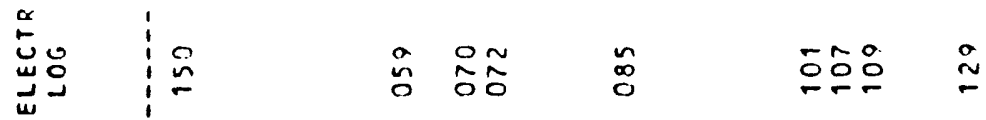
这怘

$\stackrel{\infty}{\longleftarrow \sim}$

$\omega$

a)

这京

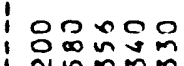

응요요

응으용ㅇㅇ

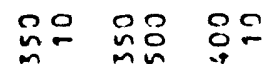

Шّ

过

王豆空至

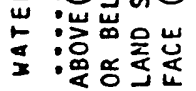

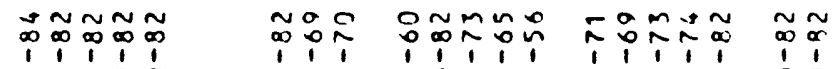

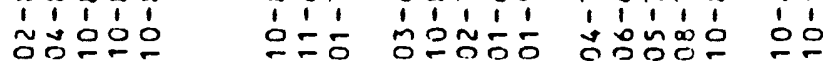

品嵒

กำ

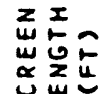

出

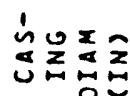

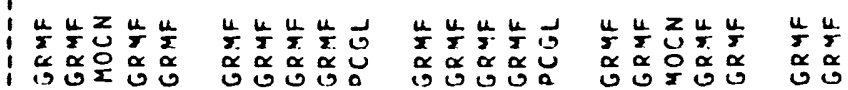

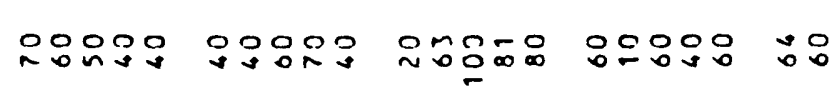

可跣

×岩岩

$\therefore$ 崖二

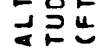

$\stackrel{2}{2}$

承品

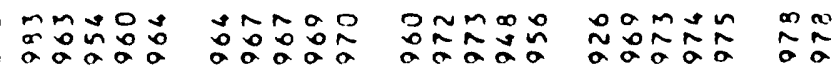

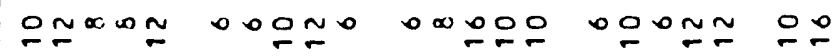

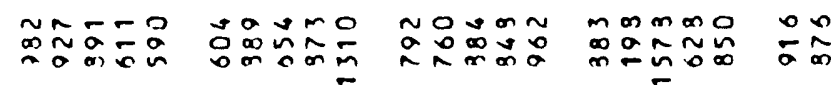

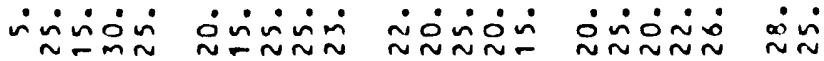

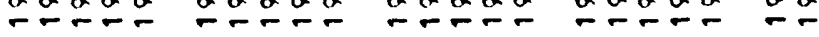

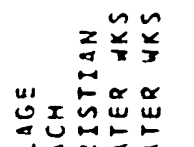

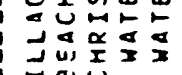

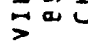

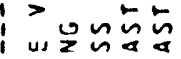

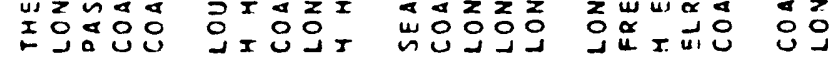

$\stackrel{\sim}{\sim} S_{\tilde{x}}$

$S_{\substack{x \\ 5}}$

$\underline{\underline{x}}$

药

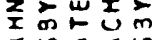

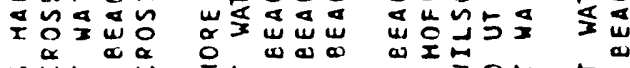

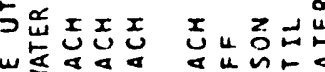

压志

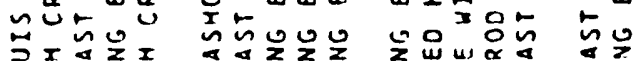

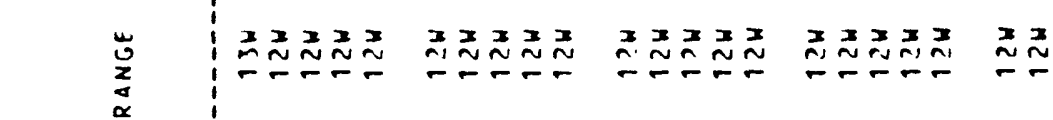

$: \frac{1}{2}$

$:$\begin{tabular}{l}
1 \\
\hdashline \\
\hdashline \\
0
\end{tabular}

is

: :

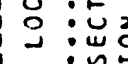

-

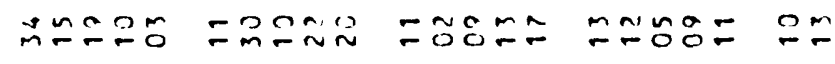

J.

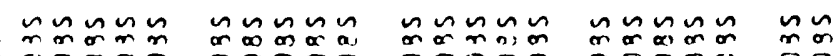

00000 00000 00000 00000 00

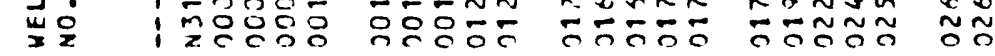


$\underset{\sim}{\alpha}$

$\underset{\leftarrow}{\omega}$

$\infty$

$\approx \stackrel{m}{\sim}$

ơ

$\infty_{0}^{\infty}$

a x

ग 0

$\vec{w}: \stackrel{w}{\sim}$

ب:

: 手余辰

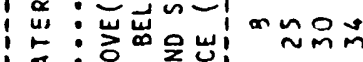

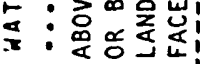

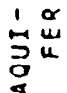

zuI

$\underset{x}{x} \leq$

ư

i

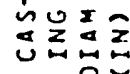

过无战

IIIII IJADU ZIJJA aDaDO Oa

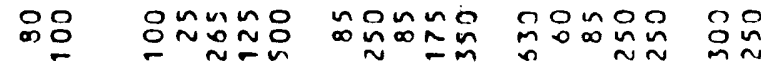

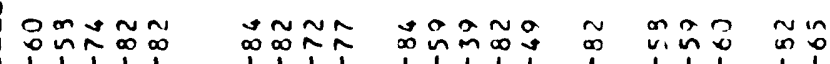

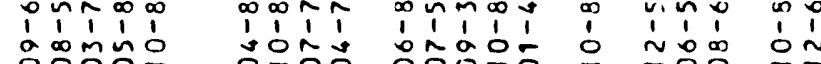

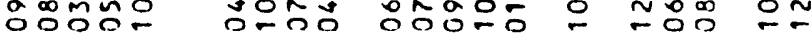

봄ำ

|⿹丁口⿹

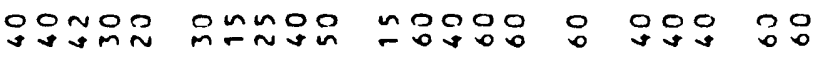

-

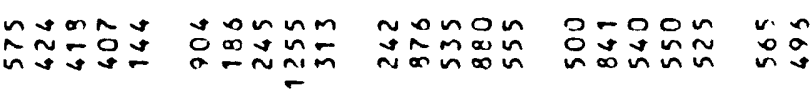

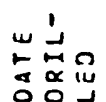

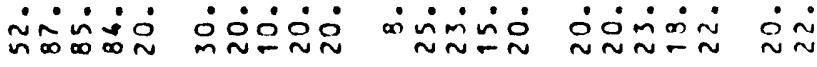

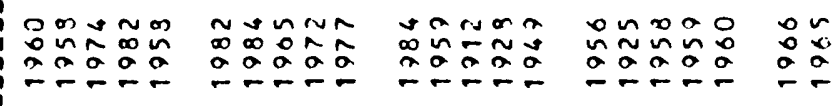

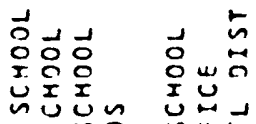

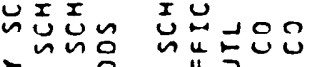

wоㅇㅇㅇ

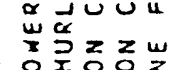

응 $\supset \alpha$

上a

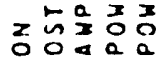

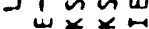

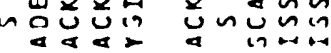

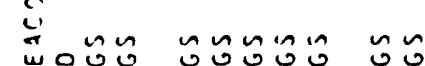

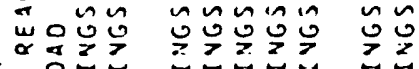

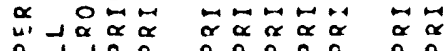
a

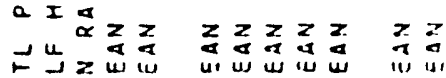

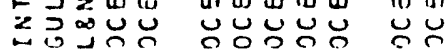

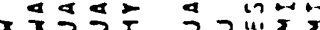

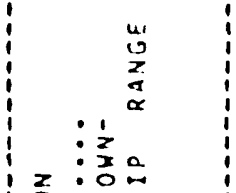

ż:논

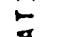

|

온:논

$: \backsim$

ज़

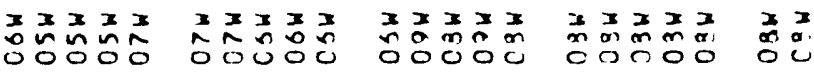

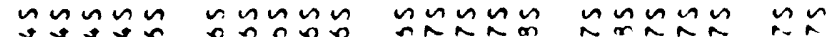

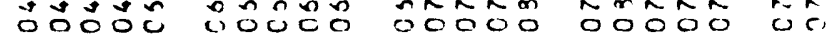

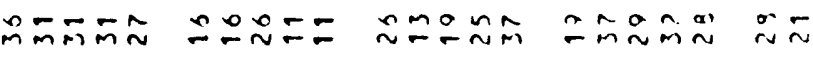

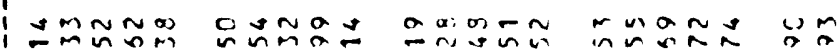

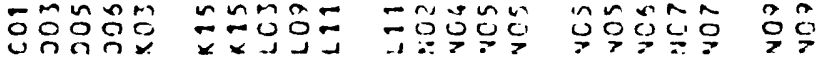




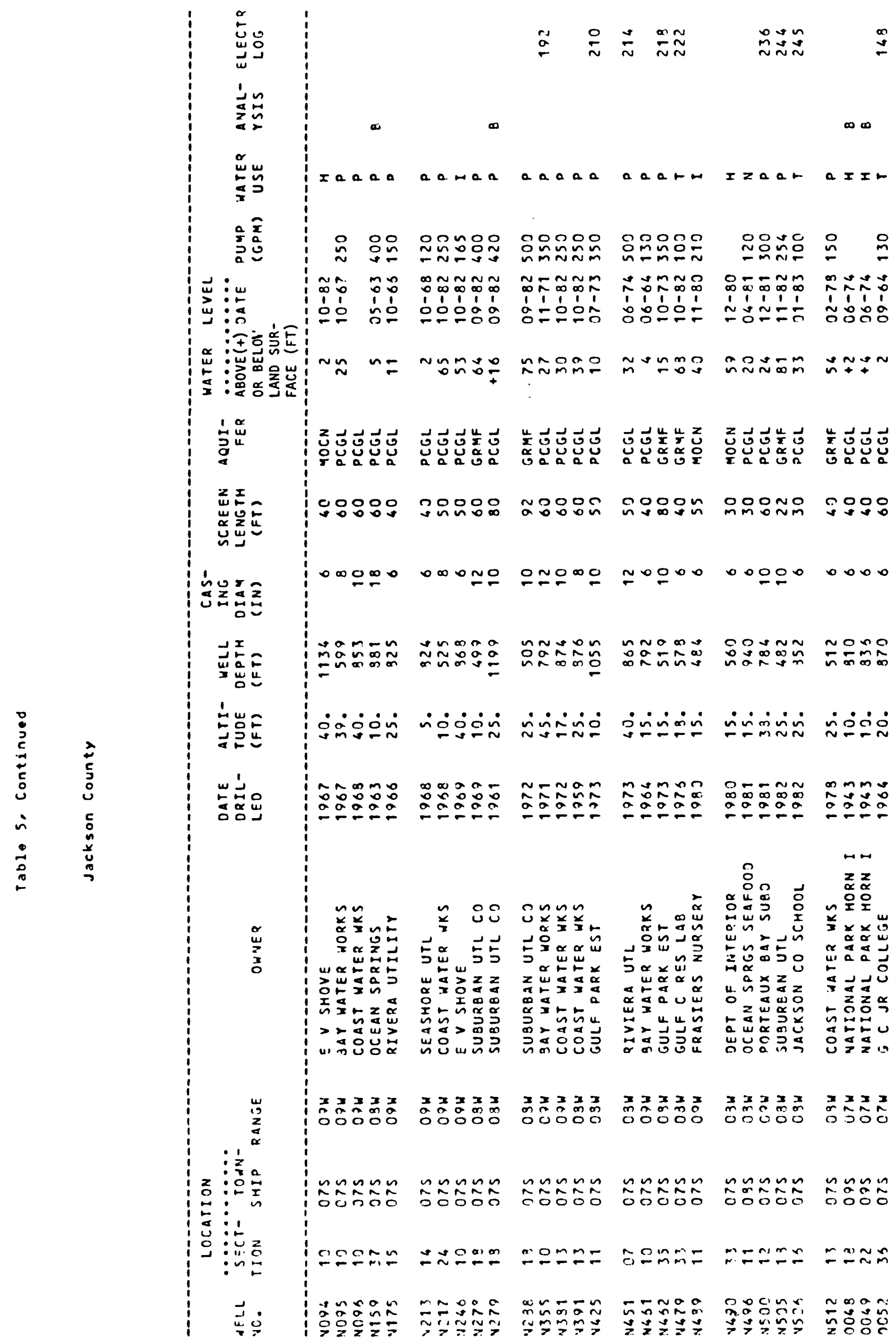




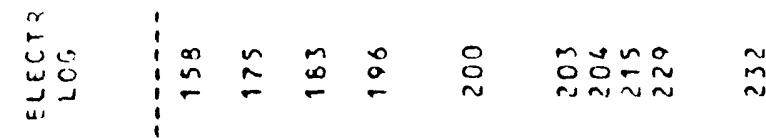

$x$

$w^{m}$ in

a a $x \leftarrow x$

$x \geq 00 I$

$x \supset \supset \supset$

$\supset \supset \supset a$

ํ.

응 으승으

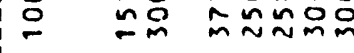

응요

잉요요

o

$\sim m$ NR

合

$\sim \sim \pm m n$

Oก⿻上丨丶

in

$n$

$\vec{w}: \underline{m}$

w:

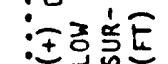

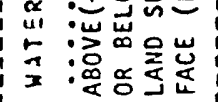

究兽 å 음 웅

=0

tomin $m$

$\tilde{n} \tilde{n}$

$\simeq$ minm

두응요

กั

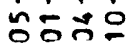

\section{دُّ}

$\overrightarrow{0} \overrightarrow{0} 己 \overrightarrow{0}$

wing $n$

$\bar{\xi}=\frac{n}{m} \quad \tilde{\sim}+\tilde{\sim} \cong$

$\sum_{u=1}=$

us y

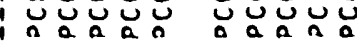

능영

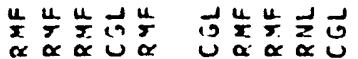

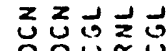
$\cos 000 \pi n$

$10 \pi+2$

nno으요

으요 MOMOO

웜웜

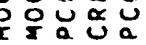

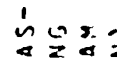

100000 ,

10000

1000

$\infty 0 \infty \infty$

$\infty \infty \infty \infty$

nomos

- $\infty \infty \infty \simeq$

ב立。

IIÑNO ÕNOẼ

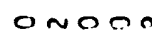

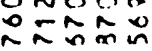

은

กํㅇㅇ

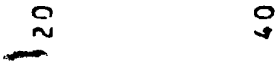

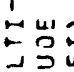

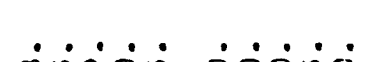

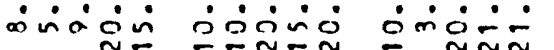

a'

至索

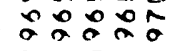

№n

응용ㅇㅇ

$-$

$\ddot{m}$

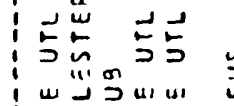

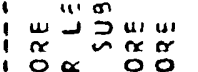

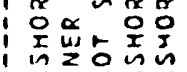

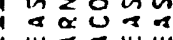

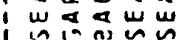

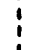

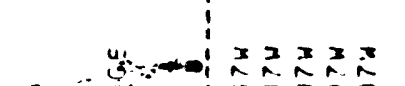

$\therefore$

$\vdots$

$z: \sum_{0}=$

$\therefore: \frac{1}{2}$

\&:

㤩:

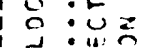

: $\because$

J.

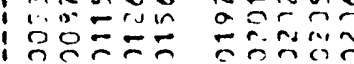

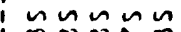

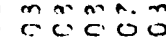

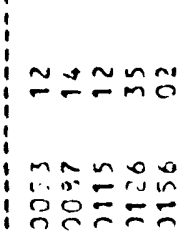

$-$
oNNN

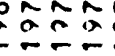

$\tilde{N} \sim \sim \sim ⿻ 上 丨$

กิำลำ

요요

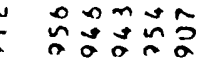

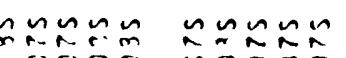

जิ

nกณนท

Cóngo

ỡ

un

wz:

uñ

인

×

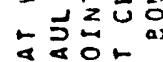

: $\dot{0}-$

$\because \dot{0} \dot{n} \dot{n}$

주원

- $200 \approx 5$

î॰

aㅕㅇ은

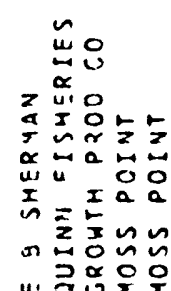

と㫖を゙

붕녕ㅇㅇㅇㅇㅇㅇㅇ

$\sim \sim \sim \sim n$ 욱오웅웅

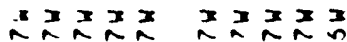

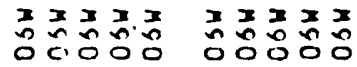

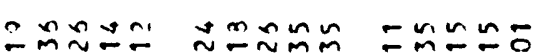

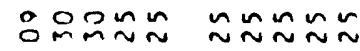

$\because 29 m a$ roña

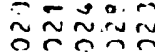

TNON?

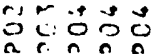

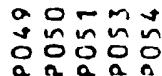




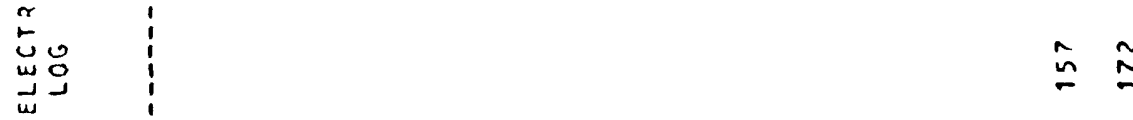

$\sum_{a}^{1} \stackrel{n}{a}$

$\infty \infty a \infty \quad \infty \quad \infty \quad \infty \quad \infty$

r.

$\underset{4}{m}$

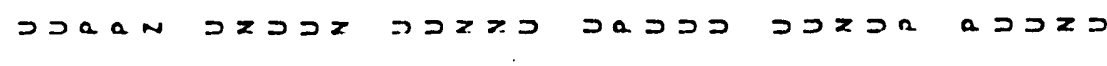

a $\bar{x}$

$\int_{0}^{2}$

So

$\stackrel{2}{\sim}$ 요

$8 \simeq 0$

웅 i 응 웅

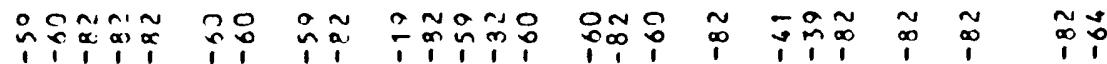

$\overrightarrow{u_{1}}: \underline{w}$

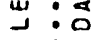

:干文宗㫐

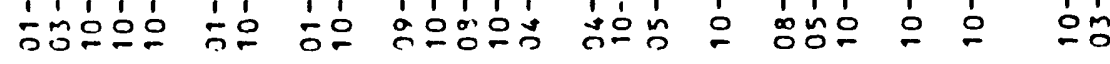

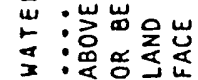

งกำaำ

$\breve{g}^{\sim}$

|

a

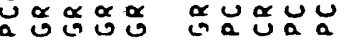

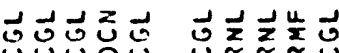

$z_{w} x_{2}$

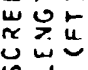

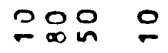

aำ

20

^ 응 용

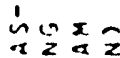

i) $2 x^{x} z$

$\exists \frac{I}{a}$

㟧㟧

$\because w=$

온는

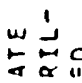

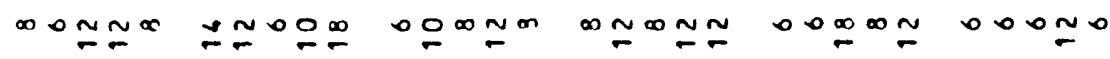

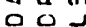

$a m \sim n \sim ⿻ 上 丨$

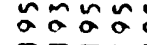

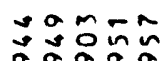

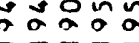

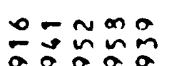

åmã

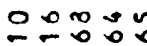

둥ㅇㅇ

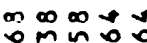

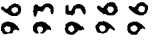

$\underset{x}{a} \stackrel{a}{0}$

崖尔品

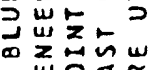

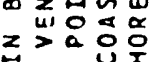

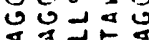

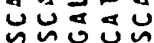

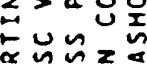

mmáa a a a a

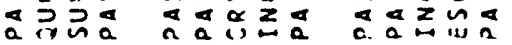

这题虽出

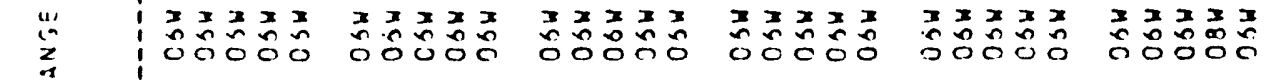

$\vdots$

$z: \vdots$

:

泣

인

in

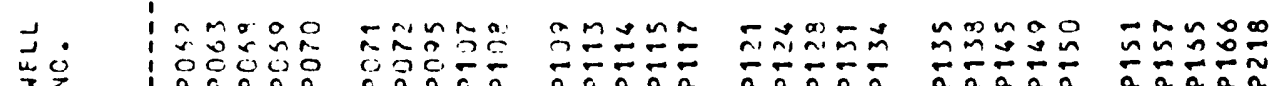




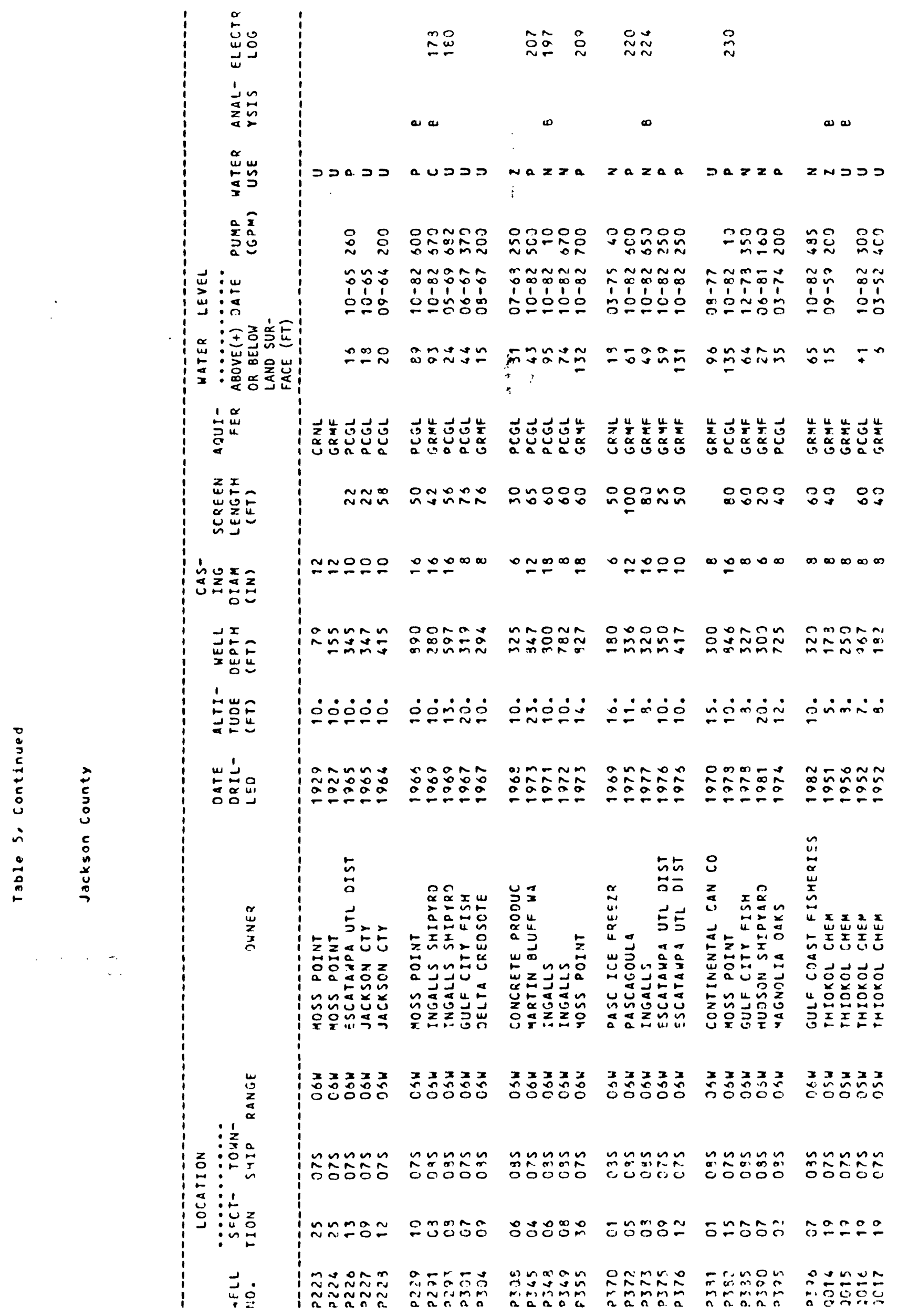




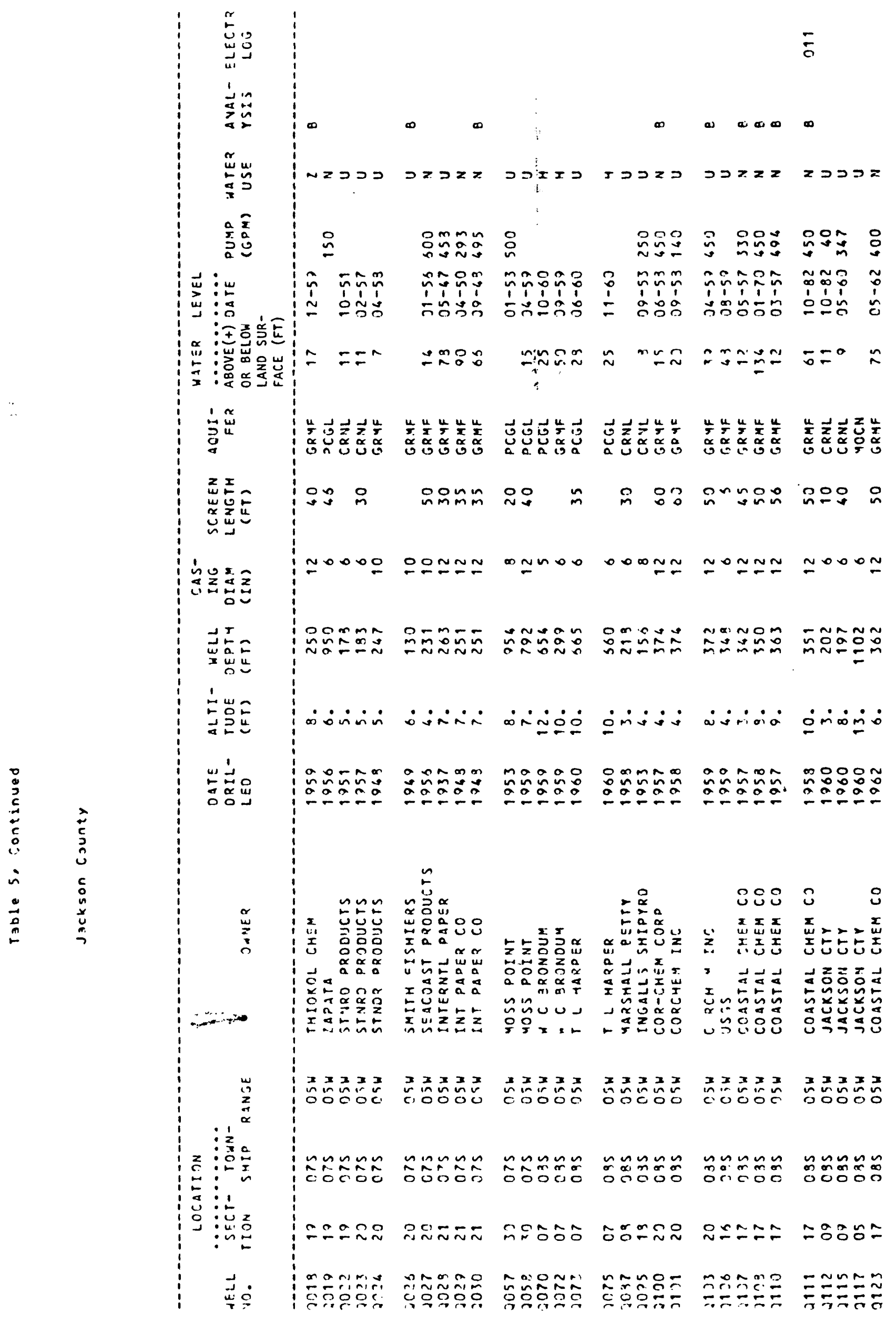




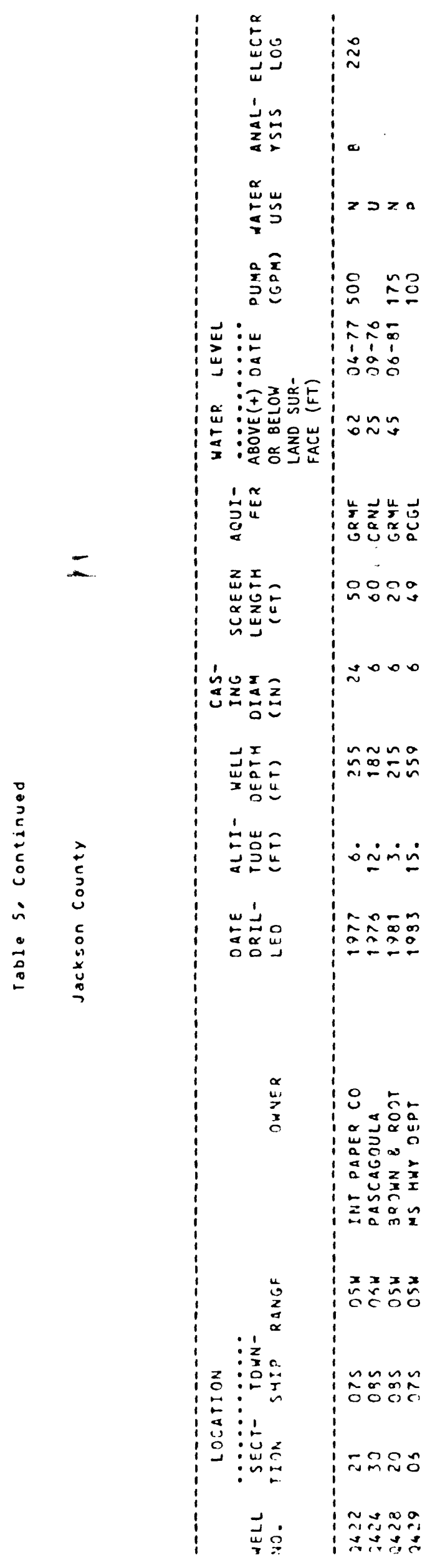


Aquifer Hydraulic Chracteristics

The hydraulic characteristics of aquifers, determined by means of pumping tests or by estimates, are used to plan well construction and to provide a basis for management studies. Wells can be designed for the most efficient operation where planning is based on known characteristics. The most economical and efficient planning for ground-water production can be done by basing distribution of wells and withdrawals on studies of aquifer hydraulic characteristics or, more effectively, by aquifer digital modeling.

Aquifers in the coastal area are characterized by great variation from place to place in transmissivity (thei capacity to transmit water). Transmissivity is the product of hydraulic conductivity (a function of the size and arrangement and interconnection of pore openings) and aquifer thickness. Newcome (1971) reported that values as determined by pumping tests in the coastal area range for transmissivity from 1,800 to $26,000 \mathrm{ft}^{2} / \mathrm{d}$; for hydraulic conductivity, from 26 to $230 \mathrm{ft} / \mathrm{d}$; and for storage coefficients for confined aquifers in the coastal area, from 0.0001 to 0.0007 , averaging about 0.0003 . Storage coefficients can be used in the calculation of the volume of water derived from compaction of the aquifer and associated clay beds as pressure is lowered.

Aquifer characteristics determined through pumping tests are specific for the aquifer in which the well is screened and for the vicinity of the pumped well. In the coastal area, the freshwater section commonly includes several aquifers, and the transmissivity of the aquifer system equals the combined value for all aquifers.

The freshwater zone thickens and individual sand beds become thicker and more numerous from east to west. Hydraulic conductivity values generally are higher for thicker beds of sand (Payne, 1968, p. 6), a concept supported by values for hydraulic conductivity found in the coastal counties (table 6). Average values are $66 \mathrm{ft} / \mathrm{d}$ for Jackson County, $126 \mathrm{ft} / \mathrm{d}$ for Harrison County, and $115 \mathrm{ft} / \mathrm{d}$ for Hancock County. Using data from table 6, thickness of the freshwater zone from Gandl (1982), and cumulative sand (aquifer) thickness from geophysical logs, average calculated values for transmissivity along latitude $30^{\circ} 30^{\circ}$ follow:

\begin{tabular}{|c|c|c|c|c|}
\hline & $\begin{array}{l}\text { Thick- } \\
\text { ness of } \\
\text { freshwater } \\
\text { zone } \\
\text { (feet) }\end{array}$ & $\begin{array}{l}\text { Cumu- } \\
\text { lative } \\
\text { sand } \\
\text { thickness } \\
\text { (feet) }\end{array}$ & $\begin{array}{l}\text { Hydrau- } \\
\text { lic con- } \\
\text { ductivity } \\
(\mathrm{ft} / \mathrm{d})\end{array}$ & $\begin{array}{l}\text { Trans- } \\
\text { missivity } \\
\left(f t^{2} / d\right)\end{array}$ \\
\hline $\begin{array}{l}\text { Hancock County } \\
\text { Harrison County } \\
\text { Jackson County }\end{array}$ & $\begin{array}{l}2,800 \\
2,400 \\
1,600\end{array}$ & $\begin{array}{l}930 \\
800 \\
530\end{array}$ & $\begin{array}{r}115 \\
126 \\
66\end{array}$ & $\begin{array}{r}107,000 \\
101,000 \\
35,000\end{array}$ \\
\hline
\end{tabular}


Water-bearing units: CRNL, Citronelle formation, GRMr, Graham ferry Formation; PCGL, Pascagoula Formation MOCN, Miocene Serles, undifferentiated

\begin{tabular}{|c|c|c|c|c|c|c|c|c|c|c|c|c|c|c|}
\hline \multirow[t]{2}{*}{$\begin{array}{l}\text { WELL } \\
\text { NO. }\end{array}$} & OANER & DATE & DEPTH & $\begin{array}{l}\text { AQUI- } \\
\text { FER }\end{array}$ & $\begin{array}{l}\text { AQUI- } \\
\text { FER } \\
\text { THICK- } \\
\text { NESS: }\end{array}$ & $\begin{array}{l}\text { SCREEN } \\
\text { LENGTH }\end{array}$ & $\begin{array}{l}\text { PUMP. } \\
\text { PERIOO }\end{array}$ & $\begin{array}{l}\text { TEST: } \\
\text { YIEIS }\end{array}$ & $\begin{array}{l}\text { SPEC. } \\
\text { CAPA- } \\
C I T Y \\
1 ; 1 M / F T\end{array}$ & $\begin{array}{l}\text { TRANS- } \\
\text { MISSI- } \\
\text { IIIIITY }\end{array}$ & $\begin{array}{l}\text { PERNEA- } \\
\text { BILITY }\end{array}$ & $\begin{array}{l}\text { STOR. } \\
\text { COEF. }\end{array}$ & $\begin{array}{l}\text { TRANS- } \\
\text { MISS- } \\
\text { IVITY }\end{array}$ & $\begin{array}{l}\text { HYDRO. } \\
\text { CON- } \\
\text { DICT- } \\
\text { IVITY }\end{array}$ \\
\hline & & & $\pi$ & & $F T$ & $F T$ & HRS & GPM & 1 - DAY & GPD/FT & $\mathrm{GPO} / \mathrm{FT}^{2}$ & & $F T / D$ & $\mathrm{FT} / \mathrm{D}$ \\
\hline
\end{tabular}

$\begin{array}{llrrrr}\text { FOOS } & \text { NSTL } & 1965 & 644 & \text { MOCN } & 110 \\ \text { HOO4 } & \text { NSTL } & 1964 & 1873 & \text { MOON } & 180 \\ \text { HOO6 } & \text { NSTL } & 1964 & 1695 & \text { MOCN } & 120 \\ \text { HOOT } & \text { NSTL } & 1964 & 1434 & \text { MOCN } & 87 \\ \text { HOOB } & \text { NSTL } & 1965 & 672 & \text { MOCN } & 170 \\ \text { HO11 } & \text { NSTL } & 1967 & 676 & \text { GRMF } & 60 \\ \text { HO12 } & \text { NSTL } & 1967 & 599 & \text { GRMF } & 70 \\ \text { HO13 } & \text { NSTL } & 1967 & 491 & \text { GRMF } & 41 \\ \text { HO14 } & \text { NSTL } & 1968 & 144 & \text { CRNL } & 60 \\ \text { HO34 } & \text { L W BROOKS } & 1965 & 1323 & \text { MOCN } & 80\end{array}$

HANCOCK COUNTY

\begin{tabular}{|c|c|c|c|c|}
\hline $\cos 8$ & EXFER FOREST & 1965 & 638 & MOON \\
\hline $\cos 1$ & SAUCIER UTIL & 1971 & 776 & $M O C N$ \\
\hline 6019 & U S FISH HATCH & 1965 & 429 & GRMF \\
\hline 6020 & U S FISH HATCH & 1965 & 431 & GRMF \\
\hline 6024 & U S FISH MATCH & 1965 & 790 & MOCN \\
\hline L002 & GUIFPORT & 1964 & 815 & MOCN \\
\hline 2014 & GULFPORT & 1964 & 763 & MOCN \\
\hline L015 & GULFPORT & 1964 & 752 & $\mathrm{MOCH}$ \\
\hline 2016 & GULFPORT & 1966 & Bis & MOCN \\
\hline 1.017 & GUIFPORT & 1966 & 848 & MOCN \\
\hline 2034 & NATIONAL, TANK & 196,8 & SBA & GRMF \\
\hline 2035 & PLUMMER-DEDEAUX & $196, \mathrm{~A}$ & 730 & $\operatorname{MOCN}$ \\
\hline LOHA & GULFPORT AIRPRT & 1764 & 645 & GRMF \\
\hline 2116 & ORANGE GRONE & 1758 & 437 & GRMF \\
\hline 4147 & GULFPORT & 196,6 & 953 & MOCN \\
\hline 2149 & GULFPORT & 1966 & 1242 & PCGL \\
\hline 216,0 & U S NAVY & 1965 & 1196 & PCGL \\
\hline L.161 & U S NAVY & 1965 & 850 & MOCN \\
\hline 4162 & US NAVY & 1365 & 357 & MOON \\
\hline 4002 & R:IOX: & 1364 & 1207 & PCGL \\
\hline 9004 & AlLOX: & 196,4 & 1200 & PCGL \\
\hline MO23 & MISS POWEA CO & 1964 & 755 & MOCN \\
\hline MO24 & MISS POWFR CO & 196,4 & B45 & MOCN \\
\hline mo40 & COAST WATER WKS & 145,5 & 654 & GRE \\
\hline 9049 & REICHHOLO CHIM & 1965 & 745 & MOCN \\
\hline MO64 & U S AIR FORCE & 1964 & 620 & GRMF \\
\hline MO68 & US AIR FORCE. & 1964 & 618 & GRMF \\
\hline M075 & U S AIR FORCE. & 1964 & 610 & GRMF \\
\hline 9076 & US AIR FORCE. & 1964 & 630 & GRMF \\
\hline M078 & US AIR FORCE & 1964 & 641 & GRMF \\
\hline M079 & US AIR FORCE & 196,4 & 640 & GRMF \\
\hline M115 & BI LOXI & 1964 & 1226 & PCGL \\
\hline M119 & BILOXI & 1954 & 1182 & PCGL \\
\hline $\mathrm{K} 147$ & MAVAR PACKING & 1966 & 945 & MOCN \\
\hline $\mathrm{NOO}_{3}$ & PASS CHRISTIAN & 196,6 & 1111 & PCGL \\
\hline 0006 & PASS CHRISTIAN & 1966 & B91 & $\mathrm{MCN}$ \\
\hline 0008 & COAST WATER WKS & 196,6 & 611 & GRMF \\
\hline 0011 & COAST WATER WKS & 1966 & 590 & GRMF \\
\hline
\end{tabular}

60
70
110
63
140
30
30
30
60
20

$\begin{array}{rr}2 & 300 \\ 24 & 3550 \\ 24 & 4900 \\ 30 & 1018 \\ 24 & 5000 \\ 8 & 141 \\ 8 & 141 \\ 8 & 141 \\ 47 & 510 \\ 1 & 50\end{array}$

$\begin{array}{rrr}20 & 110000 & 1000 \\ 12 & 100000 & 550 \\ 26 & 200000 & 1600 \\ 15 & 81000 & 930 \\ 47 & 120000 & 700 \\ 2.7 & 46000 & 760 \\ 3.8 & 14000 & 200 \\ 6.4 & 40000 & 970 \\ 46 & 44000 & 1400\end{array}$

$\begin{array}{crr}0.0002 & 14000 & 130 \\ & 13000 & 74 \\ & 26000 & 220 \\ & 10000 & 120 \\ .0002 & 16000 & 04 \\ & 6100 & 100 \\ & 1800 & 26 \\ & 5300 & 130 \\ .0001 & 11000 & 180 \\ & 6200 & 79\end{array}$

HARRISON COUNTY

50
30
20
20
60
60
60
63
70
80
60
50
63
50
71
80
29
30
60
80
80
60
60
50
60
40
40
40
40
40
40
40
65
60
60
50
40
40

1
2
1
170
100
4
5
72
2
100
8
2
3
6
1
1
1
1
2
1
1
7
1
1
1
4
1
2
2
2
2
2
1
1
1
1
1

150
150
32
60
75
1100
965
960
975
500
602
280
860
125
665
710
822
526
500
235
460
380
317
350
527
620
560
700
740
720
800
900
200
950
74
435
340
330

17
2.9
10
6.0
31
19
12
32
25
13

$75000 \quad 1500$

ROOO 200

$64000 \quad 750$

$61000 \quad 310$

$110000 \quad 910$

$51000 \quad 850$
$85000 \quad 1400$

$85000 \quad 1400$

$55000 \quad 670$

$37000 \quad 300$

$1948000 \quad 520$

B. $\quad 70000 \quad 580$

16

27000
16000

18000
96000
66000

12

5.0

16000

$23 \quad 75000$

$13 \quad 100000$

$26 \quad 100000$

$20 \quad 120000$

$18 \quad 41000 \quad 560$

$18 \quad 100003 \quad 1000$

$\begin{array}{rrr}10 & 60000 & 1000 \\ 19 & 62000 & 960\end{array}$

$22 \quad 62000 \quad 960$

$16 \quad 73000 \quad 730$

$25 \quad 80000 \quad 1000$

1299000

17

11

94000
84000

84000
24000

140000

$11 \quad 56000$

940
1000

420
1400

1400

IACKSON COUNTY

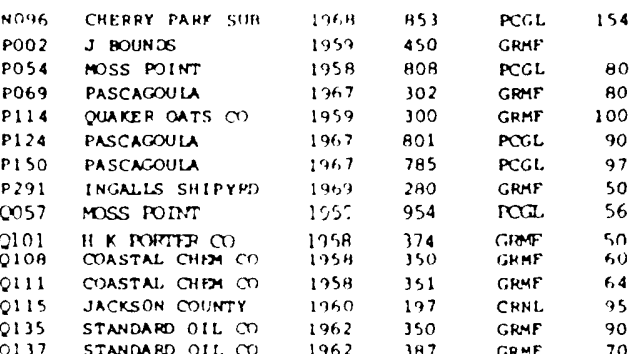

60
20
40
80
40
80
60
42
20
60
50
50
40
50
75

$\begin{array}{rrcrr}1 & 411 & & 62000 & 40 \\ 24 & 19 & 10 & 40000 & \\ 8 & 455 & 17 & 60000 & 750 \\ & 320 & 6.5 & 23000 & 280 \\ 9 & 280 & & 54000 & 540 \\ & 625 & 9.8 & 53000 & 58 \\ 1 & 840 & 19 & 52000 & 530 \\ 1 & 492 & 7.8 & 14000 & 369 \\ 8 & 500 & 13 & 60000 & 1000 \\ 8 & 1140 & 1.4 & 24000 & 480 \\ 8 & 450 & 10 & 14000 & 300 \\ 8 & 450 & 12 & 22000 & 34 \\ 456 & 347 & 11 & 60000 & 630 \\ 48 & 602 & 15 & 23000 & 25 \\ 48 & 602 & 11 & 28000 & 400\end{array}$

$\begin{array}{rrcrr}1 & 411 & & 62000 & 400 \\ 24 & 19 & 10 & 40000 & \\ 8 & 455 & 17 & 60000 & 750 \\ & 320 & 6.5 & 23000 & 280 \\ 9 & 280 & & 54000 & 540 \\ & 625 & 9.8 & 53000 & 580 \\ 1 & 840 & 19 & 52000 & 530 \\ 1 & 492 & 7.8 & 18000 & 360 \\ 8 & 500 & 13 & 60000 & 1000 \\ R & 1140 & 1.4 & 24000 & 480 \\ 8 & 430 & 10 & 14000 & 300 \\ 8 & 450 & 12 & 22000 & 340 \\ 456 & 347 & 11 & 615000 & 630 \\ 48 & 602 & 15 & 23000 & 250 \\ 48 & 602 & 11 & 28000 & 400\end{array}$

\begin{tabular}{|c|c|c|}
\hline & 10000 & 200 \\
\hline & 1000 & 26 \\
\hline & 8500 & 100 \\
\hline & 8200 & as \\
\hline .0003 & 14000 & 120 \\
\hline & 6800 & $1: 0$ \\
\hline & 11000 & 180 \\
\hline & 8600 & 130 \\
\hline .0002 & 7300 & P9 \\
\hline & 4900 & 40 \\
\hline & 6400 & 69 \\
\hline & 9300 & 77 \\
\hline .0002 & 3600 & 36 \\
\hline & 2100 & \\
\hline .0003 & 2400 & 26 \\
\hline & 12000 & 150 \\
\hline & 8800 & 200 \\
\hline & 2100 & 56 \\
\hline & 10000 & 110 \\
\hline & 13000 & 110 \\
\hline & 13000 & 130 \\
\hline .0004 & 14000 & 160 \\
\hline .0006 & 14000 & $:(6,1)$ \\
\hline & 16000 & 200 \\
\hline & 5400 & if, \\
\hline .0003 & 13000 & 130 \\
\hline .0004 & 8000 & 130 \\
\hline & 8200 & 122 \\
\hline & 8900 & 89 \\
\hline & 9700 & 77 \\
\hline & 10000 & 130 \\
\hline & 13000 & 100 \\
\hline & 12000 & 120 \\
\hline & 11000 & 140 \\
\hline & 3200 & 56 \\
\hline & 18000 & 130 \\
\hline & 7400 & 140 \\
\hline & 13000 & 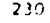 \\
\hline
\end{tabular}

$\begin{array}{lrr} & 8200 & 53 \\ .0003 & 5400 & \\ .0007 & 8000 & 107 \\ & 3000 & 33 \\ .0005 & 7200 & 72 \\ .0003 & 7000 & 73 \\ & 6900 & 71 \\ & 2400 & 48 \\ .0001 & 8000 & 140 \\ & 3700 & 64 \\ & 2400 & 42 \\ .0003 & 2900 & 15 \\ .0004 & 9000 & 44 \\ .0004 & 3000 & 14 \\ 0002 & 3700 & 43\end{array}$




\section{Water-Level Changes}

Water-level changes in shallow aquifers in or near recharge areas are seasonal in Mississippi, reflecting changes in precipitation; evaporation, and transpiration by vegetation. Water levels generally are highest in late spring and lowest in fall. Water levels in all confined aquifers have shown a long-term downward trend--the result of pumping for public, industrial, and military water supplies. Regional waterlevel declines averaging less than 2 feet per year are the result of pumping widely dispersed small wells, discharge of uncontrolled flowing wells, response to the stress of distant pumping centers, and natural discharge. Pronounced water-level declines have occurred where large withdrawals are made for public, industrial, and military water systems (Harvey and others 1965; Newcome and others, 1968; Wasson, 1978). Water-level records show that the largest decline in the coastal area is in the Pascagoula-Moss point area where the potentiometric surface declined about 136 feet from about 16 feet above sea level in 1939 to about 120 feet below sea level by 1977--the result of large withdrawals and relatively low aquifer transmissive capacity (Wasson, 1978, p. 22 and fig. 9). The shallow Citronelle aquifer, however, declined less than 20 feet during the same period (fig. 20). Moderate water-level declines have occurred at Biloxi and Gulfport. Recent measurements by the U.S. Geological Survey show that the lowest static water levels in the Biloxi-Gulfport area are now about 50 feet below sea level (B. E. Wasson, personal commun., 1984)--a decline averaging about 100 feet from the earliest levels reported (Newcome and others, 1968, p. 66). Hydrographs of representative observation wells in the coastal area are shown in figures 21, 22, and 23. Areas of significant water-level declines in the Miocene aquifer system are shown in figure 18.

\section{Saltwater Encroachment}

Saltwater encroachment (or intrusion) into ground-water bodies is commoniy considered to be a situation wherein aquifers are in direct hydraulic contact with the sea or salty estuarine water and where, as a result of head differences, saltwater moves into freshwater aquifers. Another source of saltwater is connate water--water that was trapped in the sediments at the time of depositon (Glossary of Geology, 1972).

The extent of saltwater encroachment in the Mississippi coastal area is unknown. The deepest water wells on the barrier islands do not penetrate to the base of freshwater and therefore the chemical character of water in the deeper aquifers underlying and south of Mississippi Sound has not been determined. Anomolous occurrences of saline water observed over 40 years ago in deep wells in several localities along the coast were attributed to connate water by Brown and others (1944, p. 91 ).

The shallow unconfined aquifers along the Mississippi Sound and those underlying and in hydraulic connection with coastal streams and estuaries are subject to saltwater intrusion from Mississippi Sound and from saltwater wedges that penetrate upstream (Bednar, 1978a, 1978b, and 1979; Shattles, 1973). 


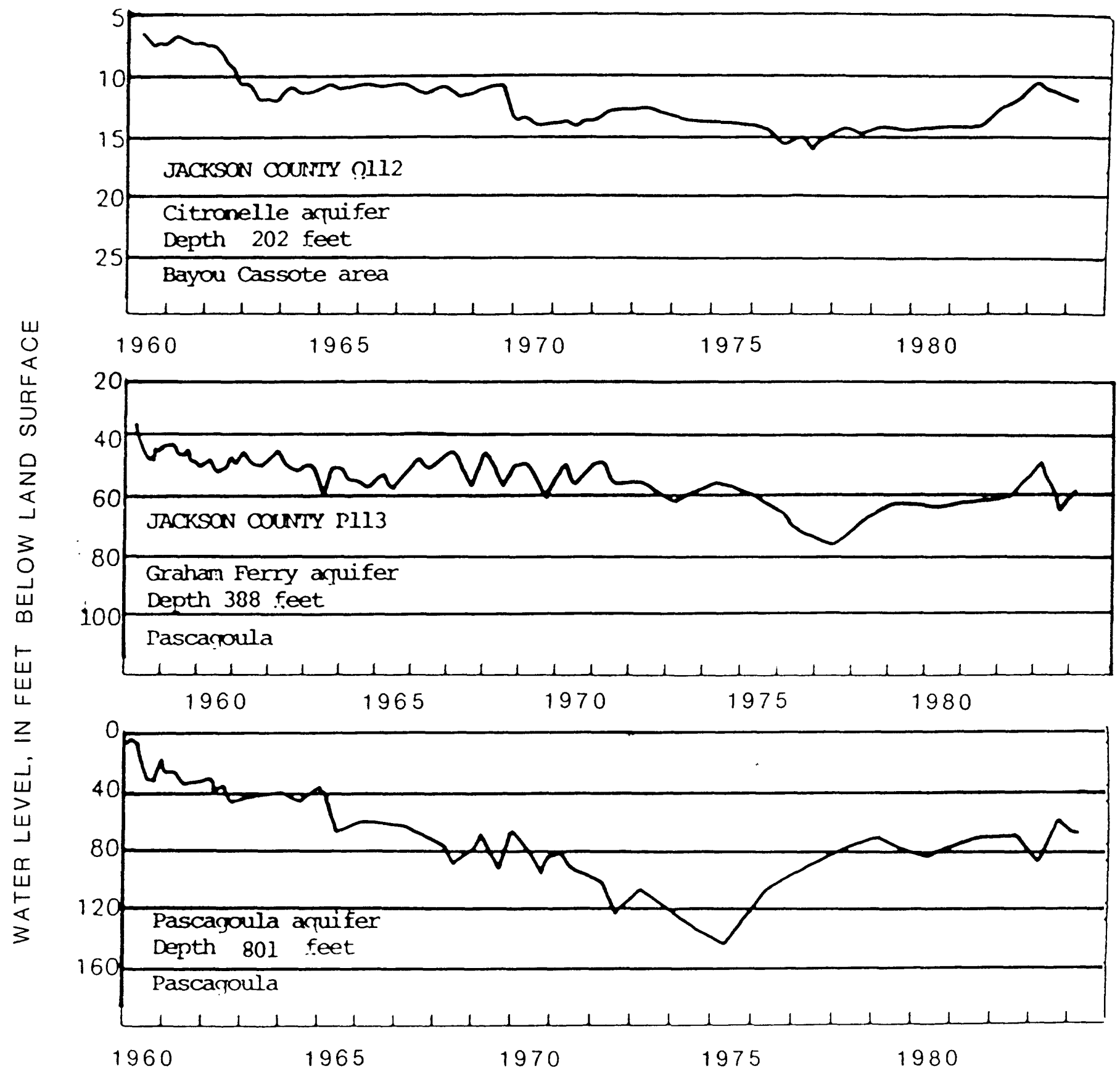

Figure 20.--Hydrographs showing water-level changes in representative wells in the Moss Point-Pascagoula area. 


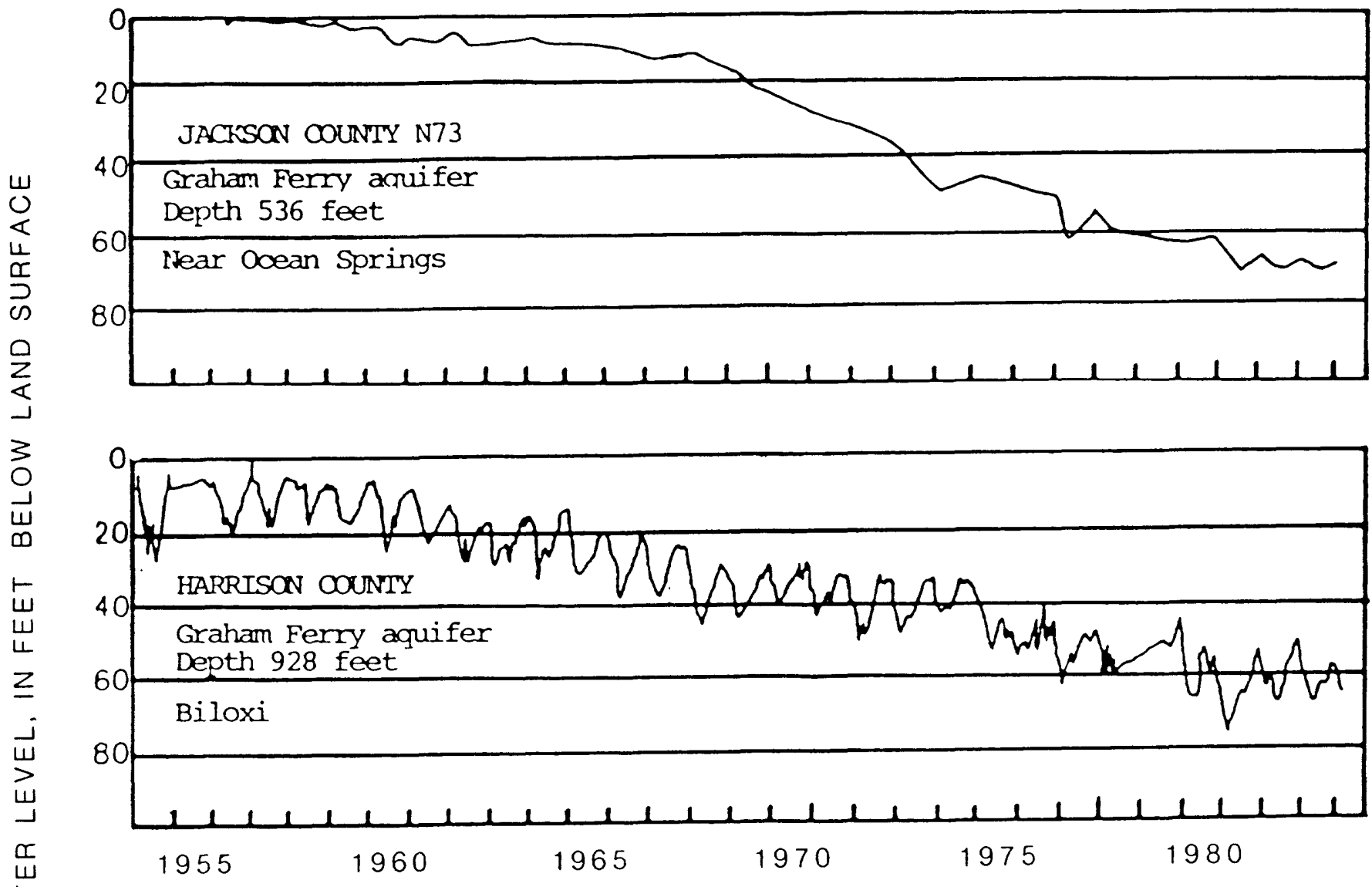

Figure 21.--Hydrographs showing water-level changes in representative well in the Biloxi-Ocean Springs area. 


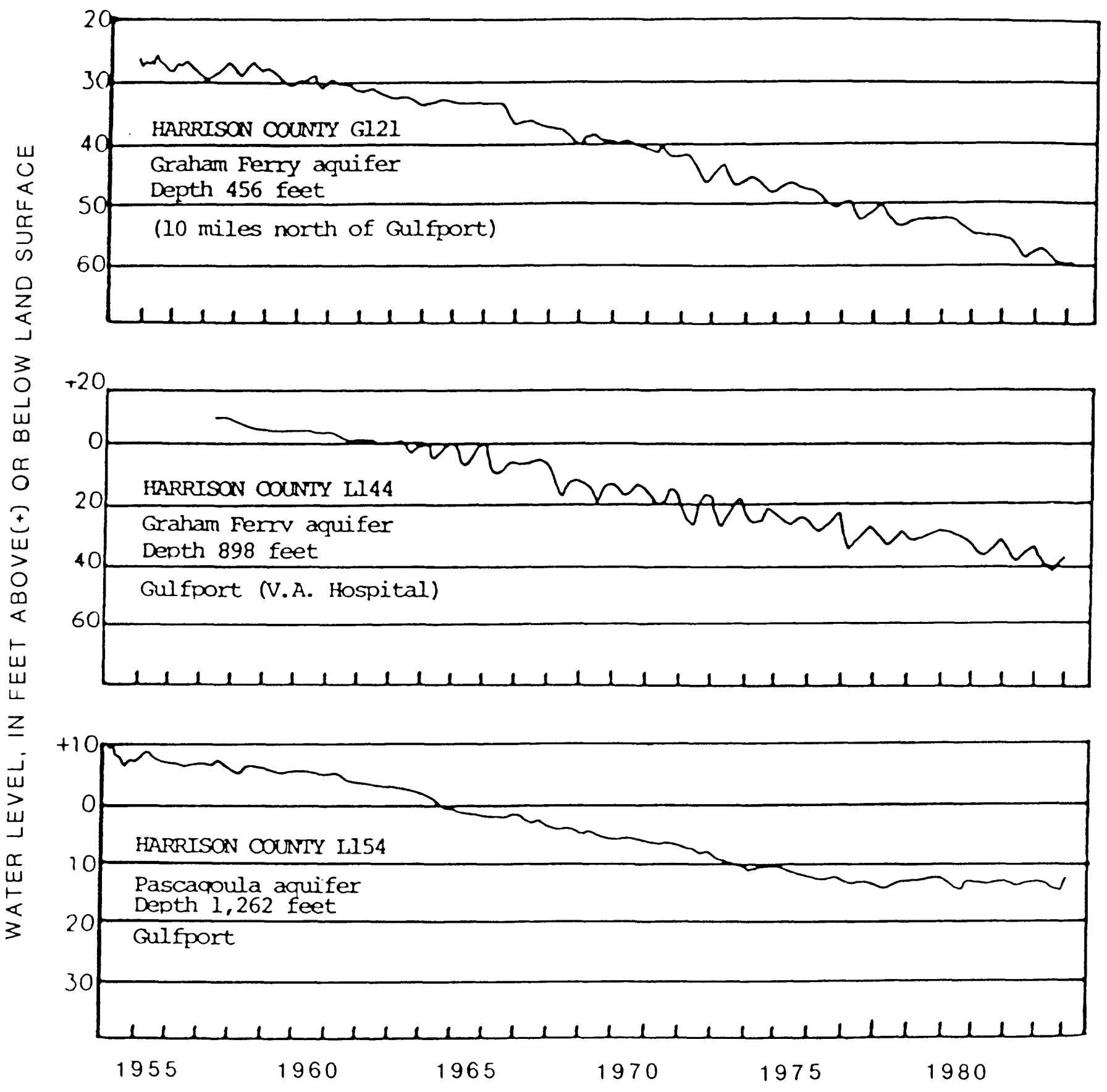

Figure 22.-Hydrographs showing water-level changes in representative wells in the Gulfport area. 


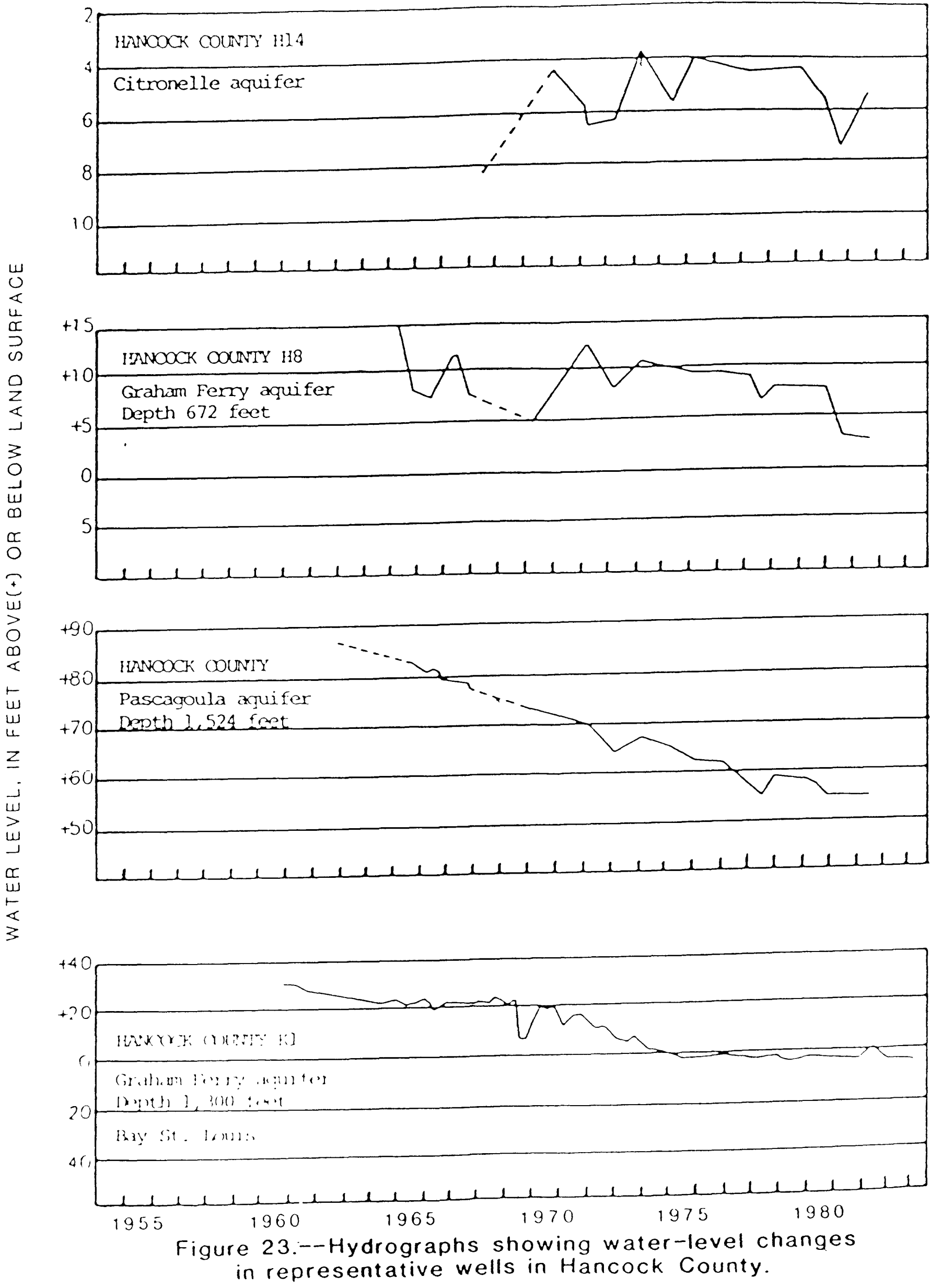




\section{Ground-Water Quality}

The statistical summary of data in Appendix $B$ gives a comparison of ground-water quality in the major freshwater aquifers and shows the range in the concentrations of chemical-constituents and the physical properties of water in the respective aquifers. Water in the Citronelle and Miocene aquifers is generally of good quality and is satisfactory for municipal use and many industrial purposes (Boswell, 1979; Newcome, 1975). The amount of water presently withdrawn from alluvial and terrace deposits is small and water-quality data are sparse.

Wells tapping the Citronelle and Miocene aquifers produce water that is comparatively low in dissolved-solids (concentrations less than $500 \mathrm{mg} / \mathrm{L})$ and that ranges from soft to moderately hard. Median $\mathrm{pH}$, sodium, and alkalinity values indicate that soft, sodium bicarbonatetype water predominates in the deeper Miocene aquifers. Median pH values in the three-county area range from 6.7 to 8.6 units for water in the Citronelle aquifer and 8.0 to 8.6 units for water in the Miocene aquifer.

Median iron values range from 10 to 45 micrograms per 1 iter (ug/L) for water in the Miocene aquifers and averages $770 \mathrm{ug} / \mathrm{L}$ for water in the Citronelle aquifer in Jackson County (Appendix 3). This suggests that water users encounter fewer iron problems with water from the deeper Miocene aquifers. Median silica values for the Citronelle $(11.5 \mathrm{mg} / \mathrm{L})$ and Miocene $(17.0 \mathrm{mg} / \mathrm{L})$ aquifers in Jackson County are lower than for these aquifers in Hancock and Harrison Counties. Silica concentrations in excess of $50 \mathrm{mg} / \mathrm{L}$ are observed in the Miocene aquifer system in Harrison and Jackson Counties. Median color values for water in the Citronelle and Miocene aquifers range from 5 to 30 units. In general, maximum color values that exceed 50 units are not typical of freshwater aquifers of the coastal counties.

Maximum values for all constituents are found in water from deeper wells or wells in areas where high dissolved solids occur anomously. High dissolved solids concentrations in the deep aquifers in the southeastern part of the Pascagoula area are believed to be related to the position of the freshwater-saltwater interface in the aquifer rather than to saltwater encroachment from Mississippi Sound or the Gulf of Mexico. The freshwater-saltwater interface in the Citronelle aquifer occurs near the edges of saltwater marshes (Wasson, 1978, p. 28) or near estuaries in the Pascagoula area. Similar conditions are presumed to prevail throughout the coastal area. Anomolous occurrences of highly mineralized water in the deeper aquifers in the Biloxi-0cean Springs area has not been studies and studied.

Water temperatures in aquifers in the coastal area range from about $68^{\circ} \mathrm{F}$ (near the mean annual air temperature) in shallow aquifers to a projected maximum temperature of about $115^{\mathrm{F}}$ at a depth of 3,000 feet (fig. 24), reflecting the average geothermal gradient in the area. The temperature of water discharged from wells screened at a given depth is affected by the discharge rate of the well and the length of pumping time as the water temperature declines during movement through shallower cooler strata. 


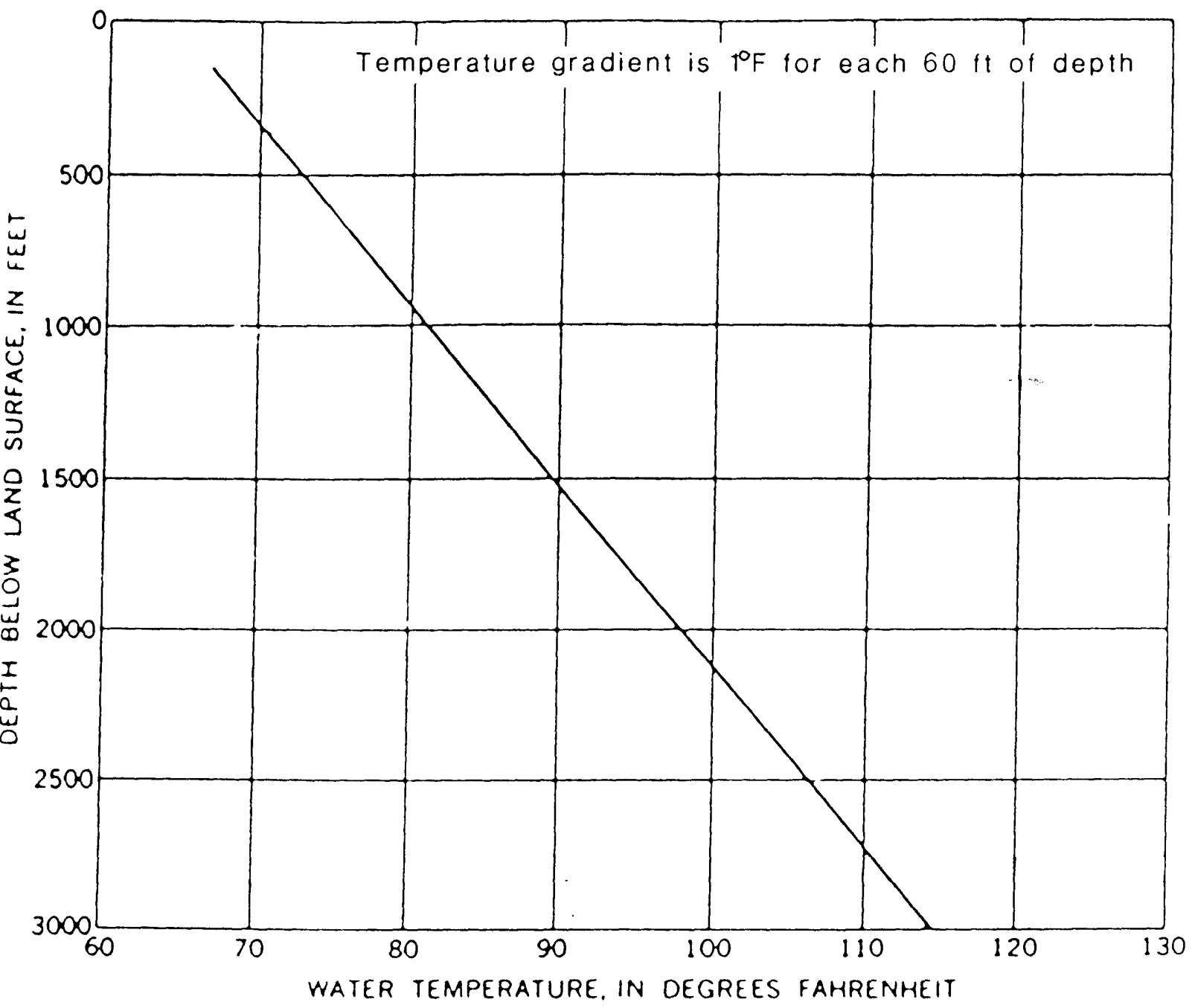

Figure 24.--Depth-temperature relation for ground water (from Newcome and others, 1968). 
The Mississippi Gulf Coast area includes Hancock, Harrison, and Jackson Counties, Mississippi Sound, and the barrier islands. Rainfall in the area averages about 60*-inches annually and temperatures during the summer months often reach $90^{\circ}$. The area is underlain by southsouthwestward dipping strata of Miocene to: Holocene age that are composed mostly of clay, silt, sand, and irregular gravely beds. The oldest exposed strata belong to the Pascagoula Formation whereas the younger Graham Ferry and the Citronelle Formations are considered to be of Pliocene age in Mississippi.

The five drainage basins in the study area discharge into the Gulf of Mexico. The major streams are the Pearl, Jourdan, Wolf, Tchoutachabouffa, Biloxi, Escatawpa, and: Pascagoula Rivers. The Pascagoula River (drainage area $9,498 \mathrm{mi}^{2}$ has an average flow at Merrill of $9,873 \mathrm{ft} 3 / \mathrm{s}$ and a flow of $1,560 \mathrm{ft} 3 / \mathrm{s}$ is exceeded 90 percent of the time. The principal tributaries in the study area are $B$ lack Creek (drainage area 1,265 $\mathrm{mi}^{2}$ ), Red Creek (drainage area $491 \mathrm{mi}^{2}$ ) and Escatawpa River (drainage area, 1,037 $\mathrm{mi}^{2}$ ).

The Tchoutacabouffa River basin (drainage area, $242 \mathrm{mi2}$ ) flows southward to a point just north of $D^{\prime}$ Iberville and then turns southwestward to enter the Back Bay of Biloxi. The principal tributaries of Tchoutacabouffa River are Tuxachanie Creek, Hurricane Creek, Bayou Billie, and Bayou Costapia. The average discharge of Tuxachanie Creek at State Highway 15 near Biloxi is $177 \mathrm{ft} 3 / \mathrm{s}$ and the flow equals or exceeds $8.6 \mathrm{ft} 3 / \mathrm{s} 90$ percent of the time.

The Biloxi River drains $271 \mathrm{mi}^{2}$, primarily in central Harrison County and discharges into Biloxi Bay. The minimum discharge observed without tide effect, $41.3 \mathrm{ft} 3 / \mathrm{s}$, occurred in 1966. The average discharge of the Biloxi River at Wortham (96.1 $\mathrm{mi}^{2}$ ) is $187 \mathrm{ft} 3 / \mathrm{s}$. The 7-day low flow at Wortham for a 10 -year recurrence is $2.2 \mathrm{ft} 3 / \mathrm{s}$ and a flow of $7.8 \mathrm{ft} 3 / \mathrm{s}$ is exceeded 90 percent of the time.

The Wolf River drains $368 \mathrm{mi} 2$ in the western part of Harrison county and the northeastern corner of Hancock County. The average flow is $668 \mathrm{ft} 3 / \mathrm{s}$ or $29.45 \mathrm{in} / \mathrm{yr}$ at the site near Landon. The 7-day low flow for a 10-year recurrence interval is $43.6 \mathrm{ft} 3 / \mathrm{s}$. A discharge of $82.3 \mathrm{ft} 3 / \mathrm{s}$ is équalled or exceeded 90 percent of the time.

Most of Hancock County is drained by the Jourdan River which discharges into St. Louis Bay. The total drainage area of $391 \mathrm{mi}^{2}$ is mostly rural with some urban areas. Tributaries to Jourdan River include Catahoula Creek, Bayou La Croix, Bayou Bacon, Rotten Bayou, Dead Tiger, Mill, and Hickory Creeks.

The Pearl River basin has a drainage area of $8,674 \mathrm{mi}^{2}$. The drainage area at Bogalusa, La., is $6,573 \mathrm{mi}^{2}$ and the average discharge is $9,671 \mathrm{ft} 3 / \mathrm{s}$. The 7 -day low flow for a 10 -year recurrence is $1,170 \mathrm{ft} 3 / \mathrm{s}$. The minimum observed discharge of $1,020 \mathrm{ft} 3 / \mathrm{s}$. 
The Mississippi Gulf Coast is subject to headwater floods caused by excessive precipitation on the drainage basins and to hurricane induced flood tides along the coast. The most destructive flood of record resulted from Hurricane Camille in August 1969.

Records of storm tides for the Mississippi coast since 1882 have been recorded at $B i l o x i$ and significant tide elevations at various points along the Mississippi coast for more than 20 hurricanes since 1893 have been recorded.

Surface water in the streams of the area is generally soft and acidic with low concentrations of dissolved solids. The concentrations of dissolved oxygen are usually greater than $4 \mathrm{mg} / \mathrm{L}$. Tannic acid is a source of high color in some streams. Suspended-sediment concentrations in streams generally are low.

The fresh ground-water zone thickens westward from the Alabama line to Hancock County and water-level differences indicate that there are several separate water-bearing zones in the coastal area. Aquifers include the Miocene aquifer system, the Citronelle aquifer and aquifers in younger alluvial and coastal deposits. The citronelle aquifers overlie in places the recharge areas of all older coastal aquifers. Minor aquifers occur in alluvial deposits in the larger valleys and in the younger coastal deposits.

The source of water in all coastal aquifers is precipitation on the outcrop areas to the north-northeast. The shallowest major aquifers along the coast are replenished in an east-west band across the northern parts of the coastal counties and the aquifers that occur at intermediate depths are recharged farther northward to about the latitude of Wiggins. The deepest freshwater aquifers receive recharge north of the latitude of Wiggins. The regional gulfward movement of ground water is interrupted by cones of depression where large volumes of water are pumped.

The base of the freshwater zone increases in depth from about 1,200 feet east of Pascagoula to more than 2,400 feet in the Gulfport area. The deepest freshwater extends to slightly below 3,000 feet in western Hancock County. Wells on the barrier islands indicate that the saltwater interface in some zones is farther to the south.

Most large wells in the coastal counties are made at depths ranging from about 400 to about 1,000 feet. A few wells exceed 1,200 feet in depth and the deepest, a 2,400-foot well, was drilled recently at Gulfport. The highest measured pumping rate was $5,865 \mathrm{gpm}$ from a 672-foot well at National Space Technology Laboratories. Values for hydraulic characteristics as determined by pumping tests in the area range from 1,800 to $26,000 \mathrm{ft} 2 / \mathrm{d}$ for transmissivity; from 26 to $230 \mathrm{ft} / \mathrm{d}$ for hydraulic conductivity; and for storage coefficients for confined aquifers in the coastal area, from 0.0001 to 0.0007 , averaging about 0.0003 . The freshwater zone thickens and individual sand beds become thicker and more numerous from east to west. 
Regional water-level declines average less than 2 feet per year. The largest decline in the coastal area is in the cone of depression in the Pascagoula-Moss Point area--to about 136 feet below sea level by 1977. Water levels in the Biloxi-Gulfport cone of depression extend as deep as about 50 feet below sea level--about 100 feet below the earliest reported levels.

The shallow unconfined aquifers in hydraulic connection with coastal streams are subject to saltwater intrusion. The extent of saltwater encroachment in the confined aquifers in the Mississippi coastal area is unknown. Anomalous occurrences of saline water observed in several localities along the coast are attributed to connate water.

Wells tapping the Citronelle and Miocene aquifers produce water that is comparatively low in dissolved-solids concentrations. Sodium bicarbonate-type water predominates in the deeper Miocene aqufiers. Silica concentrations in excess of $50 \mathrm{mg} / \mathrm{L}$ occur in Miocene aqufiers in some wells in Harrison and Jackson Counties.: Color values are generally less than 50 units.

High dissolved soids concentrations in the deep aquifers in the southeastern part of the Pascaogoula area may be related to the position of the normal freshwater-saltwater interface in the aquifer rather than to saltwater encroachment. Water temperatures in aquifers in the coastal area range from about $68^{\circ} \mathrm{F}$ in shallow aquifers to a projected maximum temperature of about $115^{\circ} \mathrm{F}$ at a depth of 3,000 feet.

All domestic and public water-supply systems use ground water; however, most of the water used in the area in terms of volume is surface water. Water use in the three counties in 1980 averaged about $770 \mathrm{Mgal} / \mathrm{d}$ of which 86 percent was brackish surface water. Freshwater use averaged about $109 \mathrm{Mgal} / \mathrm{d}$ of which $31.3 \mathrm{Mgal} / \mathrm{d}$ was for public water supplies. Fresh ground water use increased about 6 percent between 1975 and 1980; the use of surface water also increased. 
American Geological Institute, 1972, Glossary of Geology.

Bednar, G. A., 1978a, Quality... of water and dye dispersion characteristics in the Back Bay of Biloxi, Harrison County, Mississippi: U.S. Geological Survey Open-File Report 7:8-908, 62 p.

1978b, Quality of water: in Pascagoula and Escatawpa Rivers, Jackson County, Mississippi: U.S. Geological: Survey Open-File Report 78-913, $91 \mathrm{p}$.

1979, Quality of water in Bayou Cassotte and Bayou Chico, Jackson County, Mississippi: U.S. Geological Survey Open-File Report 79-913,91 p.

Belt, W. E., and others, 1945, Geologic map of Mississippi: Mississippi Geological Society, 1 sheet.

Bettandorff, J. M., 1972, Selected characteristics of Mississippi streams part 3 , coastal river basins: Mississippi Board of Water Commissioners Bullet in 72-2, $32 \mathrm{p}$.

Boswell, E. H., 1979, The Citronelle aquifers in Mississippi: U.S. Geological Survey Water-Resources Investigations 78-131, map.

Brown, G. F., Foster, V. M., Adams, R. W., Reed, E. W., and Padgett, H. D., Jr., 1944, Geology and ground-water resources of the coastal area in Mississippi: Mississippi State Geological Survey Bulletin $60,232 \mathrm{p}$.

Brahana, J. V., and Dalsin, G. J., 1977, Water for industrial development in George, Hancock, Pearl River, and Stone Counties, Mississippi: Research and Development Center Bulletin, $70 \mathrm{p}$.

Callahan, J. A., 1975, Public and industrial water supplies in southern Mississippi, 1974: Mississippi Board of Water Commissioners Bullet in $75-2,59 \mathrm{p}$.

1976, Water use in Mississippi-1975: U.S. Geological Survey WaterResources Investigations $76-125,1$ sheet.

1982, Water use in the Mississippi Gulf Coast Counties, 1900: U.S. Geological Survey Open-File Report 82-512, 13 p.

1983, Water use in Mississippi, 1980: U.S. Geological Survey OpenFile Report 83-224, 1 sheet.

Faye, R. E., 1980, Hydrologic reconnaissance of the Pascagoula and Escatawpa Rivers, Jackson County, Mississippi, May 1973 to May 1977: U.S. Geological Survey Open-File Report, 27 p.

Gand1, L. A., 1982, Characterization of aquifers designated as potential drinking-water sources in Mississippi: U.S. Geological Survey Water-Resources Investigations Open-File Report 81-550, $90 \mathrm{p}$. 
Harvey, E. J., Golden, H. G., and Jeffery, H. G., 1965, Water resources of the Pascagoula area, Mississippi: U.S. Geological Survey WaterSupply Paper 1763, 135 p.

Johnson, L. C., and Eckel,..E. C., 1904, Mississippi in Fuller, M. L., Contributions to the hydrology of the eastern United States: U.S. Geological Survey Water Supply Paper 102, 522 p.

Newcome, Roy, Jr., 1967a, Development of ground-water supplies at Mississippi Test Facility, Hancock County, Mississippi: U.S. Geological Survey Water-Supply Paper 1839-H, 28 p.

1967b, Ground-water resources of the Pascagoula River basin, Misssissippi and Alabama: U.S. Geological Survey Water-Supply Paper $1839-\mathrm{K}, 36 \mathrm{p}$.

1975, The Miocene aquifer system in Mississippi: U.S. Geological Survey Water Resources Investigations Report 46-75, 3 sheets

1971, Results of aquifer tests in Mississippi: Mississippi Board of Water Commissioners Bulet in 71-2, $44 \mathrm{p}$.

Newcome, Roy, Jr., Shattles, D. E., and Humphreys, C. P., Jr., 1968, Water for the growing needs of Harrison County, Mississippi: U.S. Geological Survey Water-Supply Paper 1856, $106 \mathrm{p}$.

Payne, J. N., 1968, Hydrologic significance of the lithofacies of the Sparta Sand in Arkansas, Louisiana, Mississippi, and Texas: U.S. Geological Survey Professional Paper 569-A, 17 p.

Rainwater, E. H., 1962, Geological history and oil and gas possibilities of Mississippi in Mississippi Geologic Research Papers-1962: Mississippi Geological Economic and Topographical Survey Bulletin $97,106 \mathrm{p}$.

Shattles, D. E., 1973, A hydrologic reconnaissance of the Pascagoula River Estuary, Mississippi in 8th Mississippi Water Resources Conference Proceedings supplement: Water Resources Research Institute, Mississippi State University, 29 p.

Shattles, D. E., and Callahan, J. A., 1970, Water-level and waterquality trends in aquifers along the Mississippi Gulf Coast, 1970: Mississippi Board of Water Commissioners Bulletin 70-1, 25 p.

Tharpe, E. J., Low-flow characteristics of Mississippi streams: Mississippi Board of Water Commissioners Bulletin 75-1, 60 p.

U.S. Department of Commerce, 1968, Climatic atlas of the United States: U.S. Government Printing office, Washington, DC.

U.S. Geological Survey, 1977, Hydrologic Unit Map-1974, State of Mississippi. 
U.S. Geological Survey, 1968, Ground-water resources of the Pat Harrison Waterway District - a compilation of basic data: U.S. Geological Survey Open-File Report, $37 \mathrm{p}$.

Wasson, B. E., 1978, Availability of additional ground-water supplies in the Pascagoula area, Mississippi: Mississippi Research and Development Bulletin, $32 \mathrm{p}$.

1980, Sources for water supplies in Mississippi: Mississippi Research and Development Bulletin, $112 \mathrm{p}$.

Wilson, K. V., and Hudson, J. W., 1969, Hurricane Camille tidal floods of August 1969 along the Gulf Coast, Mississippi: U.S. Geological Survey Hydrologic Atlas Investigations, HA-397 through HA-408, maps. 


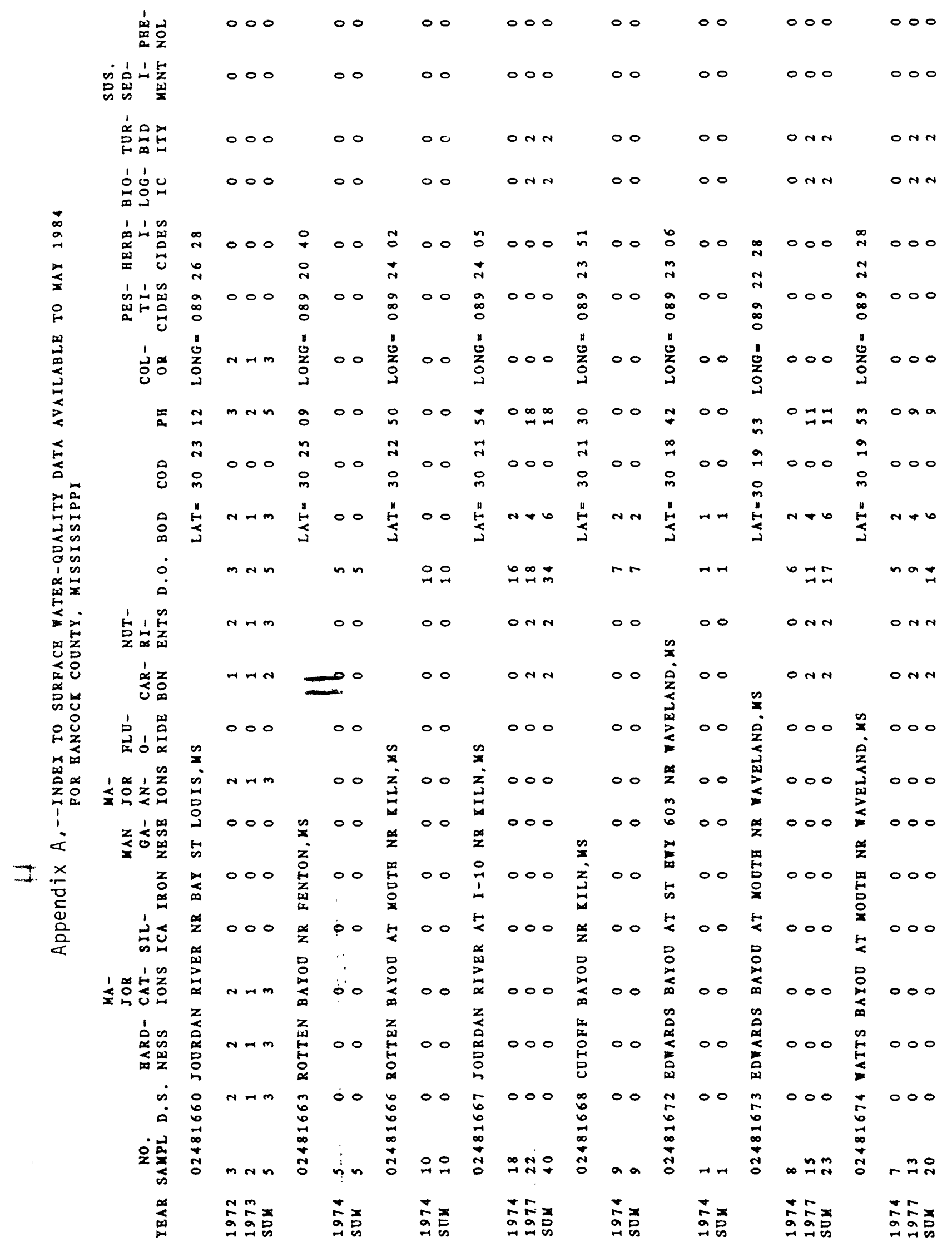




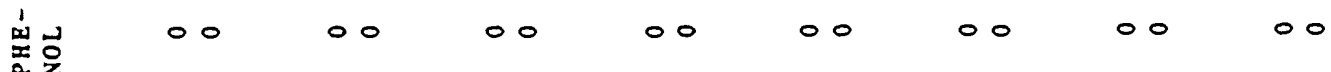

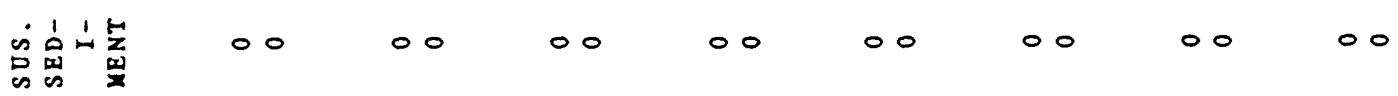

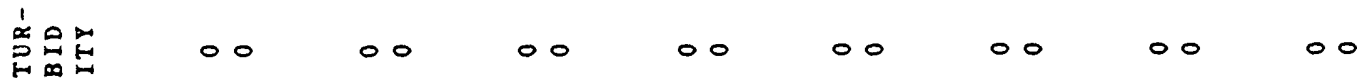

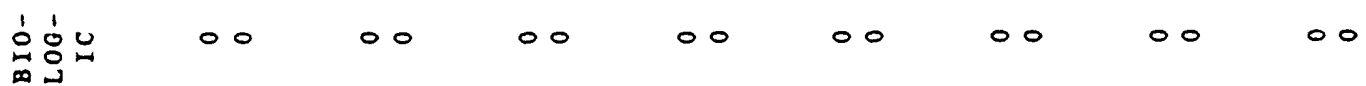

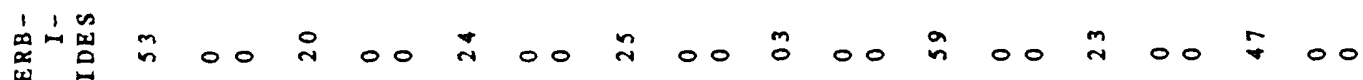

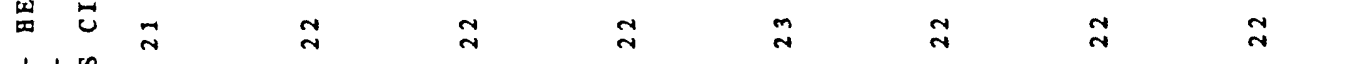

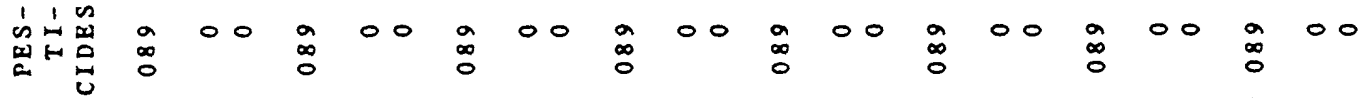

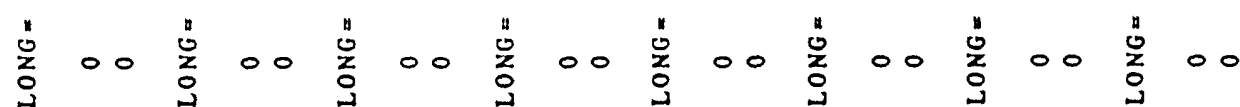

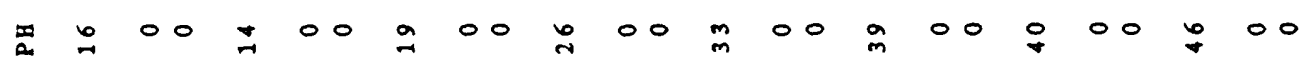
占

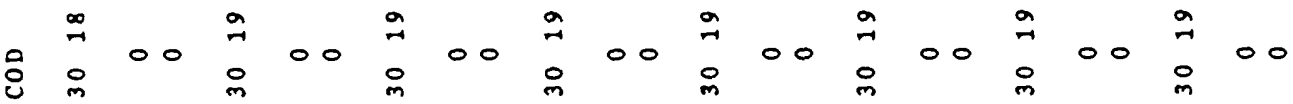

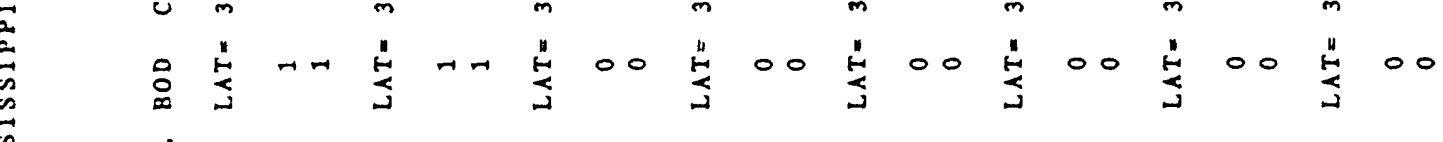

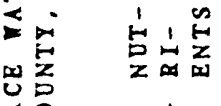
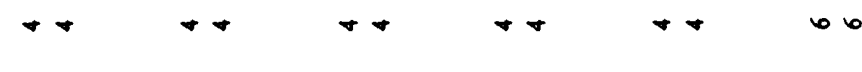

66

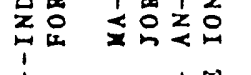

i.

$\dot{<}$

$\frac{x}{0}$

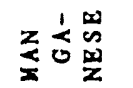

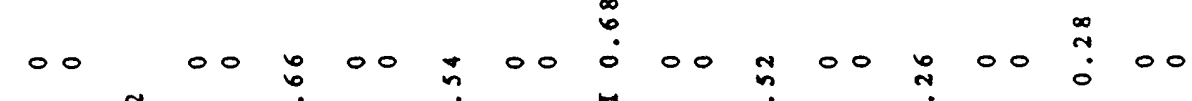
$00 \stackrel{0}{7} 00 \stackrel{0}{0} 00 \stackrel{0}{0}$

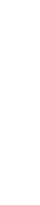




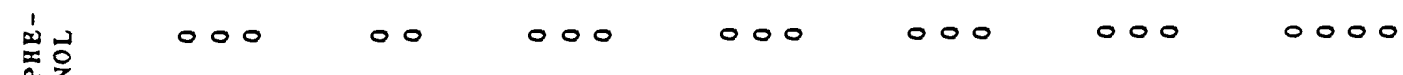

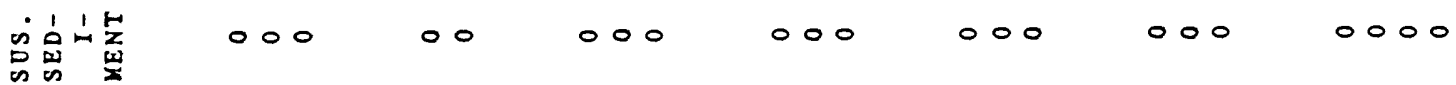

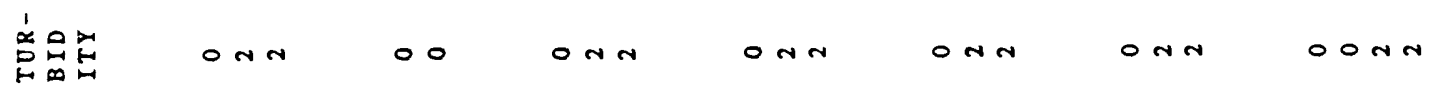

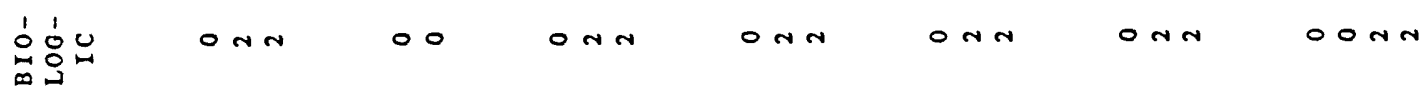

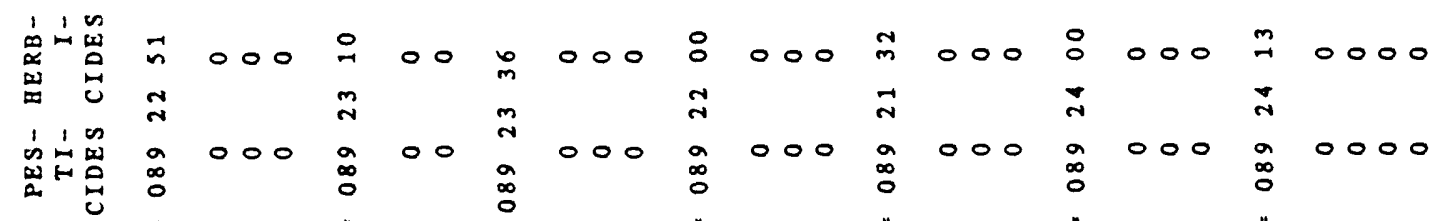

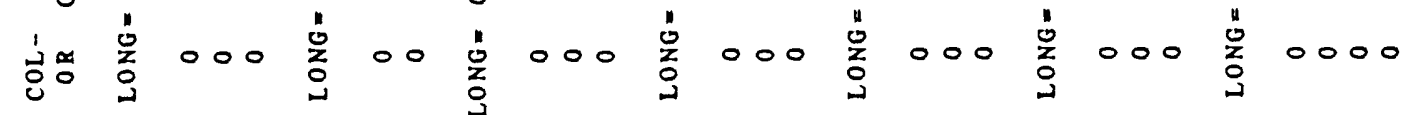

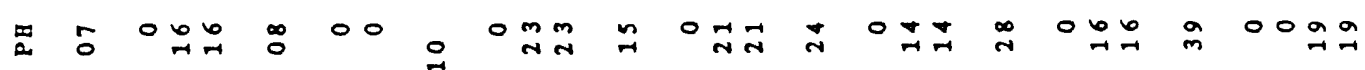

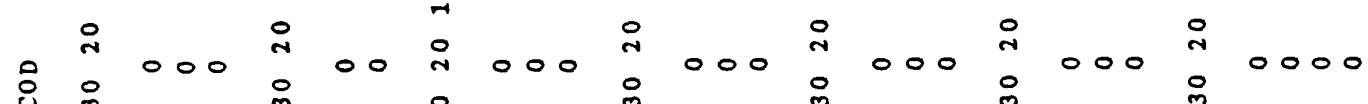
藍点

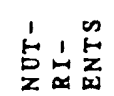

$$
-\infty m
$$$$
\infty \infty
$$

$\circ m m$

and

$- \pm \overrightarrow{4}$

$\infty \underset{0}{-4}$

의모

这z

$0 n n$

00

$0 n n$

on

on

on

odn

$04 n$

00 onh

onn

on

on

o onn

只, 品

000

00000

000

000

000

0000

武离

究造 $1 \infty 12$

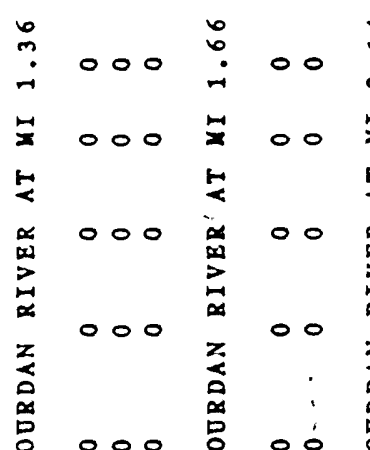
$\leq \stackrel{1}{1}$

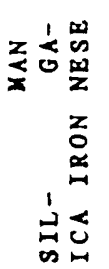

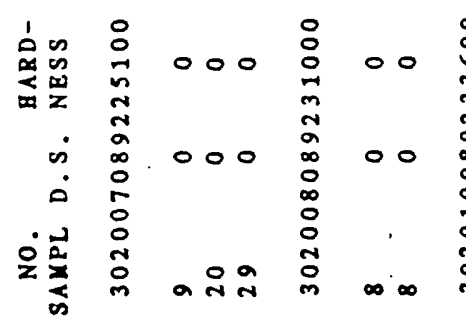

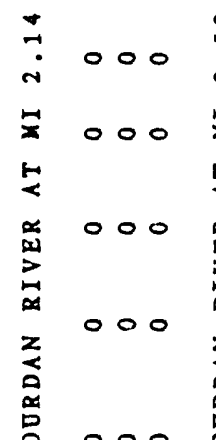

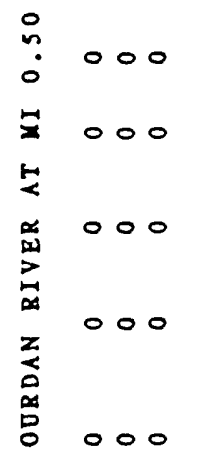

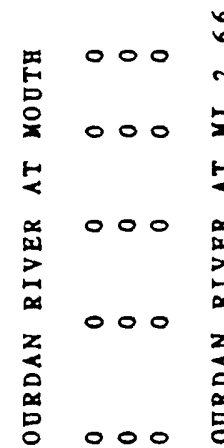

$\therefore 000 \stackrel{5}{\circ} 0000$

$<$

$x$
$\frac{x}{0}$
$\frac{0}{0}$
0
$\frac{0}{2}$
$\frac{0}{1}$

茄

동 000

000

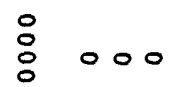

$\begin{array}{cccc}1 & & & \\ 0 & 0 & 0 & 0\end{array}$

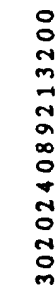

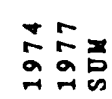

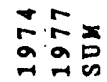

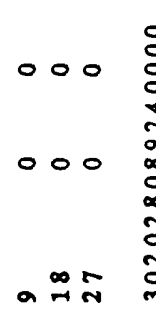

000

0000

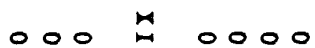
选

$000 \leqq 0000$

$00 \stackrel{D}{\circ} 0000$

:

000

0000

ant

$\infty \stackrel{\sim}{\sim}$

은 $\underset{n}{\infty}$ 


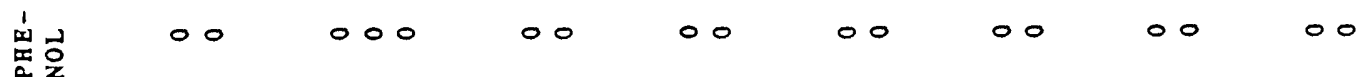

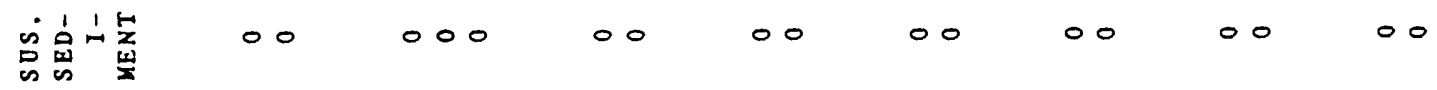

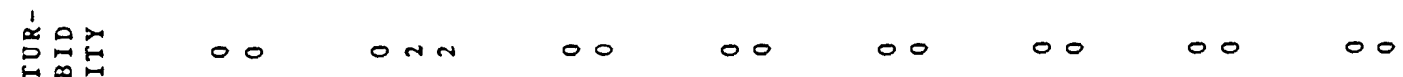
$\begin{array}{lllllll}1 & 000 & 0 & 0 & 0 & 0 & 0\end{array}$

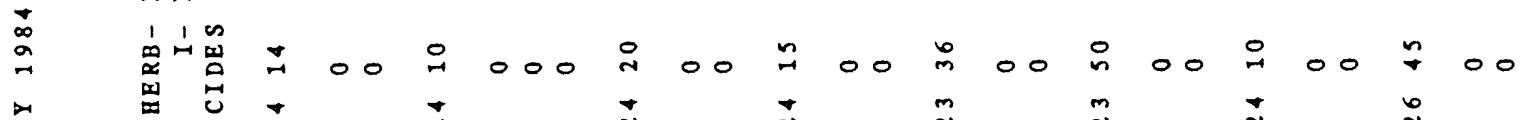$$
\underset{\nexists}{\Xi}
$$

웅

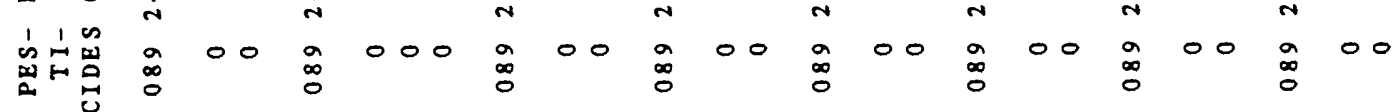

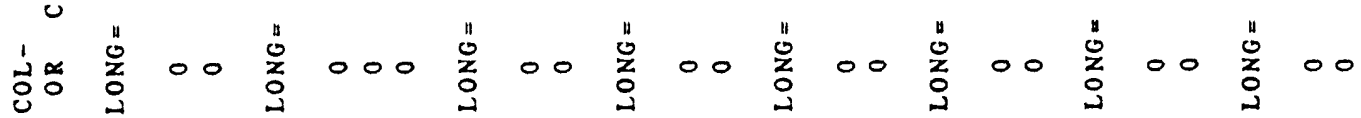

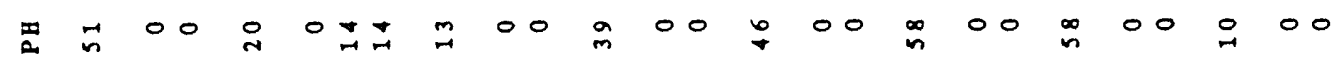

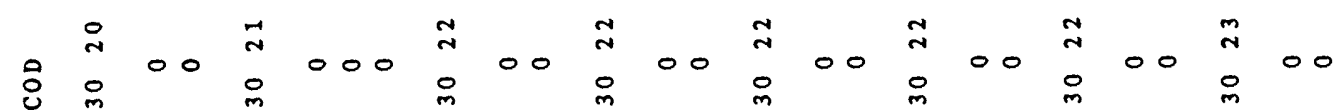
营莴 㝘

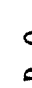

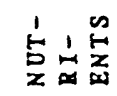
$\operatorname{ron}$ $n n$

a a

$n n$

60

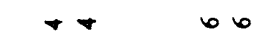

00

on

00

00

00

00

00

00 00

on

00

00

co

00

00

00

웅

压

$\dot{i}$

$x$
$x$
0
0
0
0
$\frac{0}{2}$
$\frac{1}{1}$ 尘虽交文

00

000

00

00

00

00

00

00

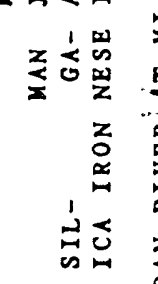

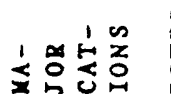

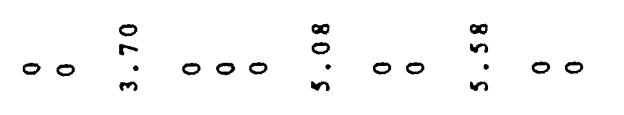

$00 \#$ I $000 \pm 00$

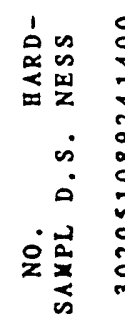

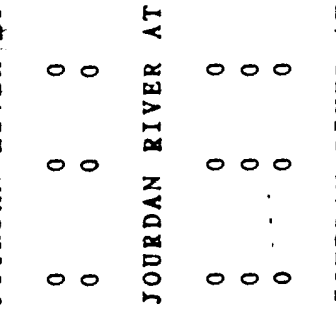

0

0

00

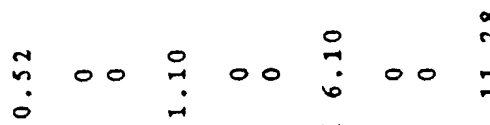

00 
ม்⿻

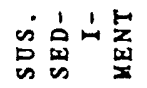

立的

ㅎำ

000000

0000

00

$\circ 0$

00

$\circ 0$

文

$\begin{array}{cc}1 & \infty \\ 0 & 0 \\ 0 & 0 \\ 0 & 0\end{array}$

的禹要

$=00.8$

m 00 i $00 \stackrel{n}{n} 00$

i

4

$\sqrt[4]{0} 0$

क

官品

$00 \quad 0$

$00 \int_{\infty}^{\infty} 00$

in

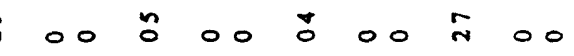

n

i

室

$\rightarrow \stackrel{a}{a}$

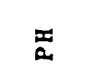

8

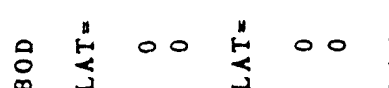

$\therefore$ rr

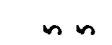

㟔岕点

0

00

选甭

00

00

00

00

00

00

00

00

00

s.

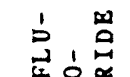

00

00

00

00

00

00

00

00

00

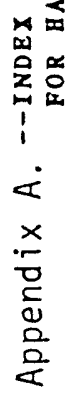

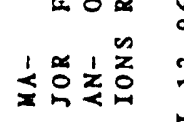

z衣售

$00 \vdots 00 \stackrel{4}{!} 00=$

$\stackrel{\infty}{i} 00 \stackrel{0}{0} 00$

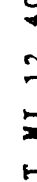

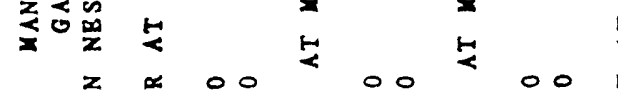

范

岕矛

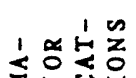

00

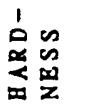

i

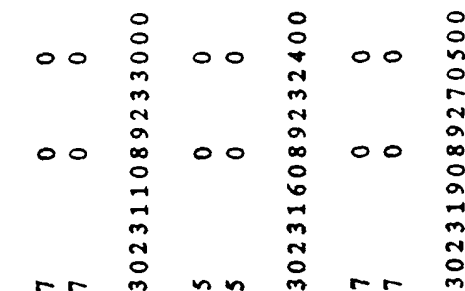

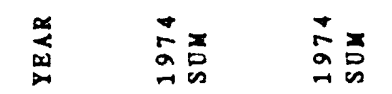

站竞

00

$\sum_{\infty}^{\infty} 00$

00
00
00
00

$: 00$

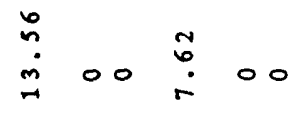

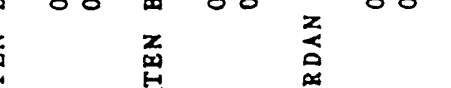

蛋

$00 \stackrel{0}{\circ} 00$

00

- 00

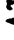

$\rightarrow 00$

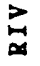

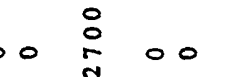

0

âd

00

00

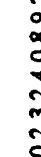

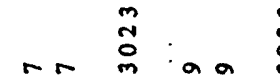

$m m$

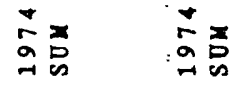

츰

옹

突言

䇏 


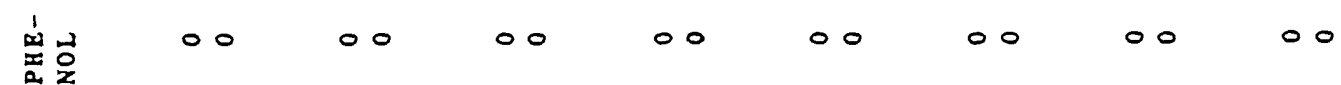

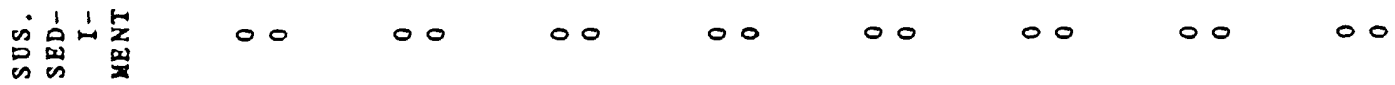

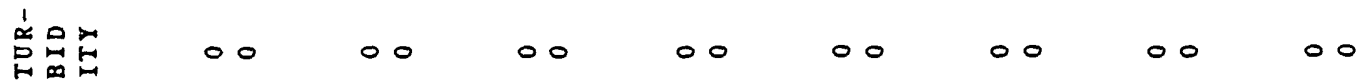

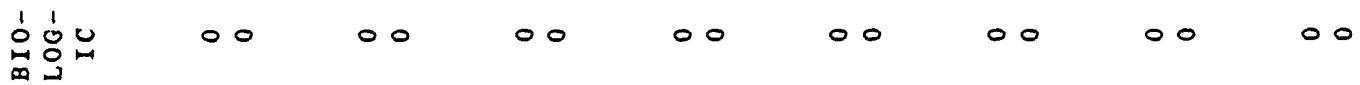

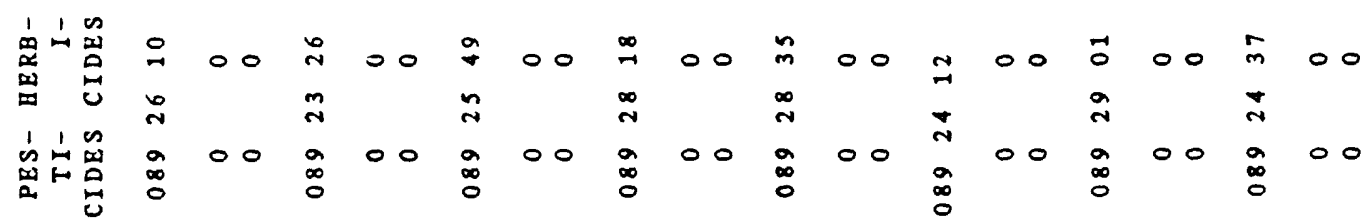
光品 E

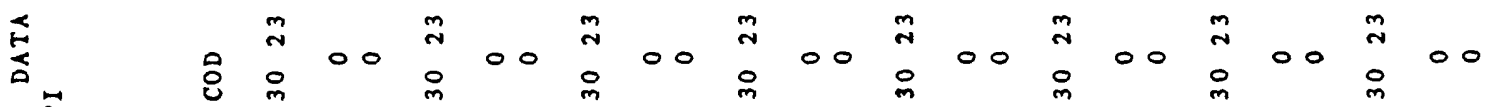
$>\vec{a}$

$\stackrel{1}{H}, \stackrel{\infty}{H}$

$00 \quad 00$

$00 \quad 00$

00

00

00

00

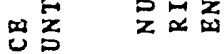

00

00

00

00

00

究

b , 嘿

$\circ 0$

$00 \quad 00$

0000

$00 \quad 00$

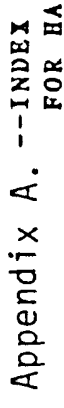

$1 x+2$

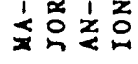

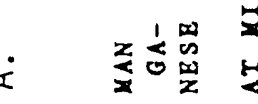

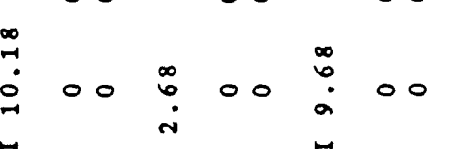

$\stackrel{\square}{\square} 00$

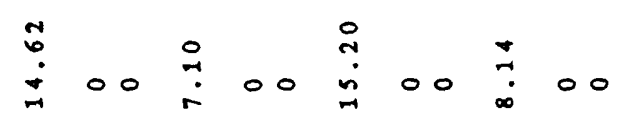

$00 \geqslant 00 \geqslant 00$

z

岁皆

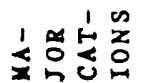

至

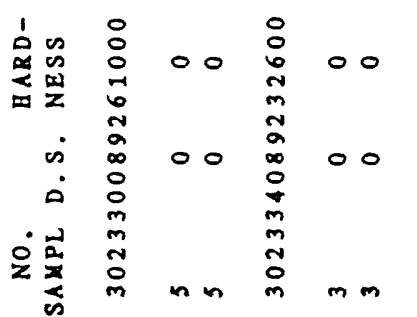

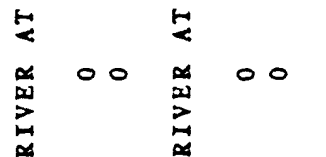

$00 \sum_{0}^{2} 00$

$\pi x \quad t x$

aำ

aำ

$\underset{\substack{t \\ 2}}{20}$

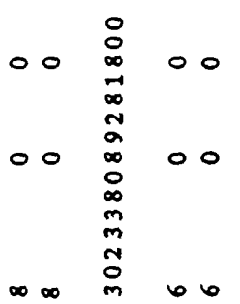

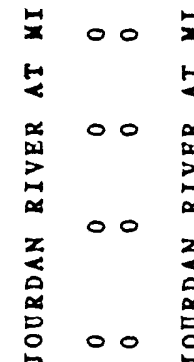

$\circ \circ$

$\$ 00$

00

0

$\geq 00$ 


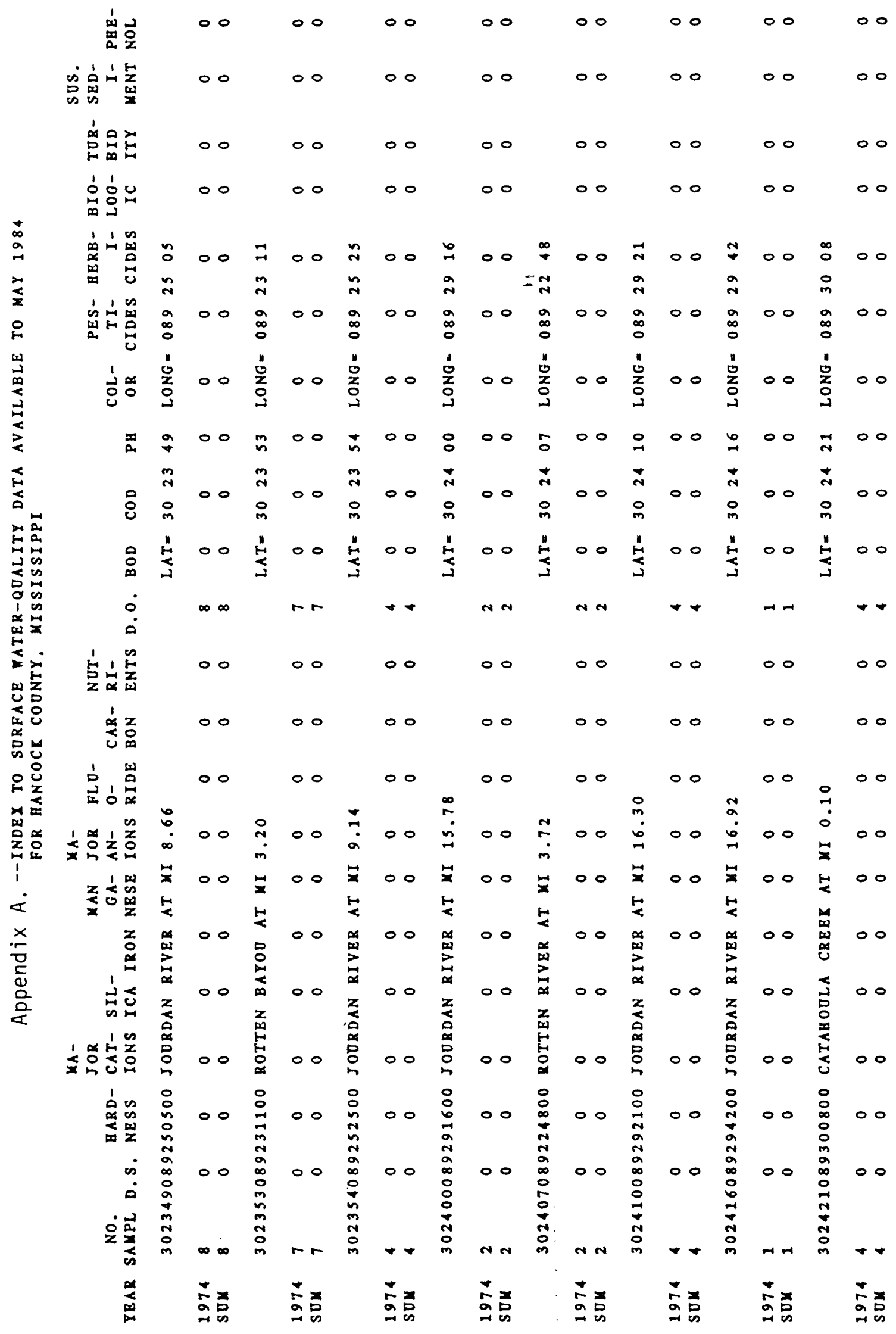




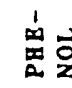

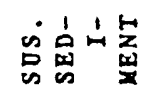

ㅎํำ

ㅎํำ

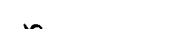

(i)

$200 \div 00 \geq$

部印

宁哭

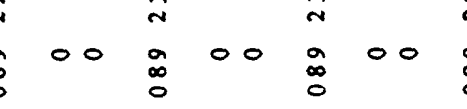

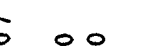

00

mano

N

ne

至

崌点点

n $00 \stackrel{n}{n}$

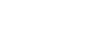

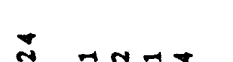
n 100

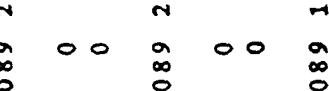

$\infty$ a

$\therefore 00$ in 00 $\circ$ s. i - $i^{i} 00 \quad \frac{i}{4}$

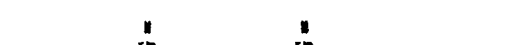
染 00 $\circ$

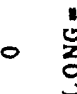
$\pi n \rightarrow+$ 2 $00 \underset{\infty}{a} 00$ $\begin{array}{llll}1 & & & 1 \\ 0 & 0 & & \\ 0 & 0 & 0 & 0\end{array}$
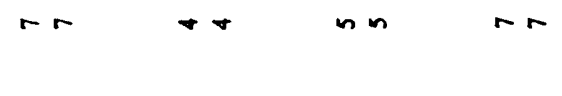

\section{0}

$\circ 0$

$\stackrel{H}{j} 00 \stackrel{n}{!}$

$\stackrel{n}{\leftrightarrows} 00+t$

$00 \stackrel{1}{0}$

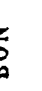

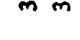

nN

$\min m$

$ㅇ ㅜ$

a a

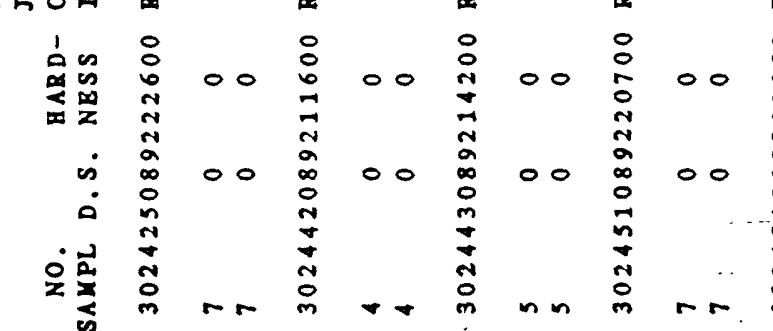

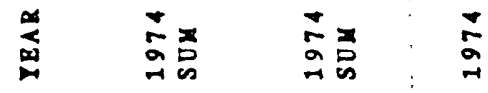

00

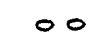

$00 \quad 00$

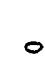

00

䇏

\section{就}

\section{$001-00$ \\ (1)}

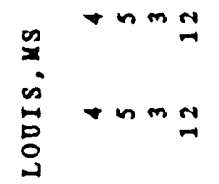

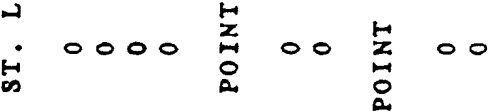

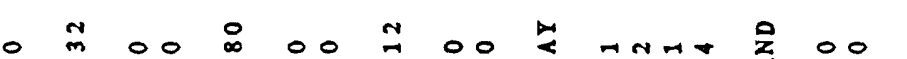

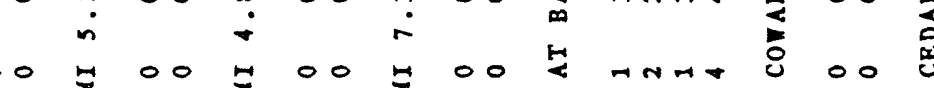

00

$00 \quad \bar{x} 00 \quad \bar{x} 00 \quad$

00 K 00 F 00 F

\&

品

뭉

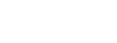

\&

00 ○

0

落 00

点 00

00

蛋 总 点

(a)

is 00 म 00

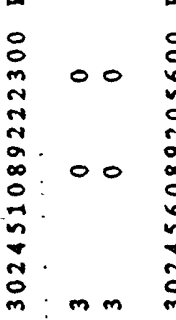

00

00

00

a
.

\section{s.}

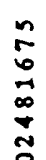

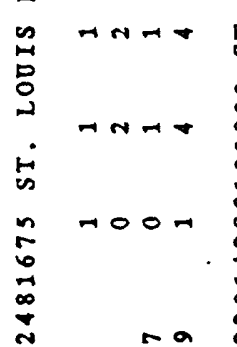

¿ $-n=\hat{~}$

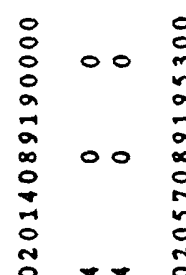

00

00

$m m$ 


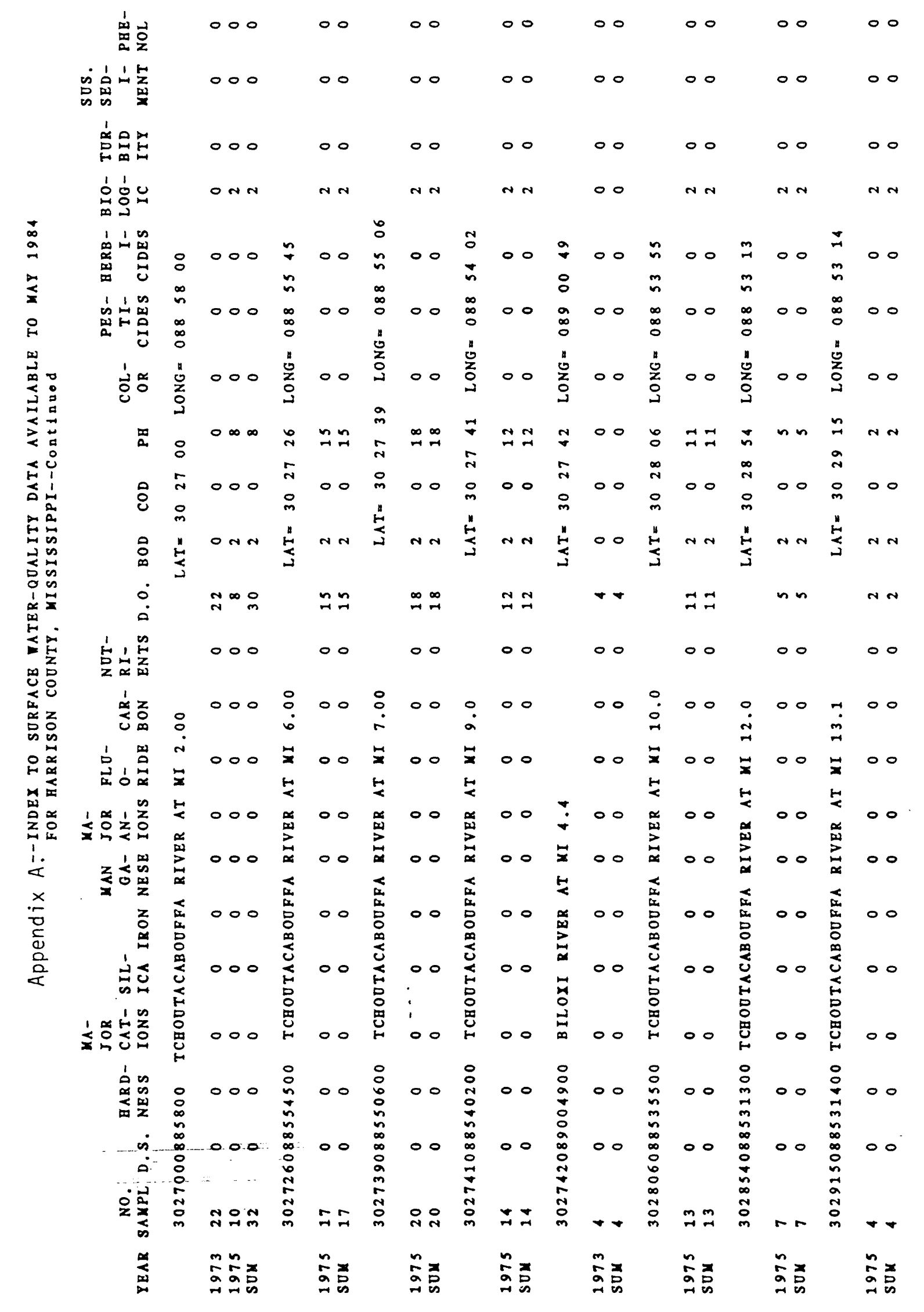




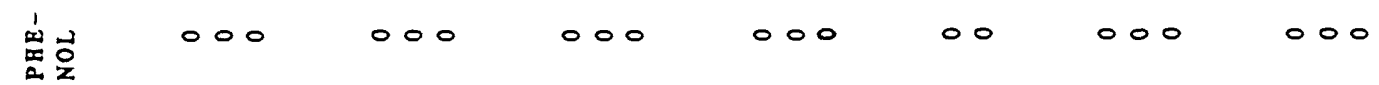

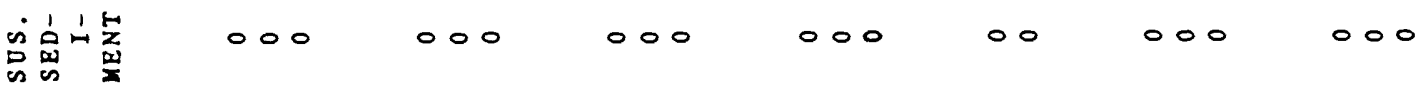

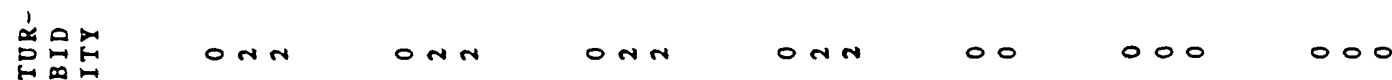

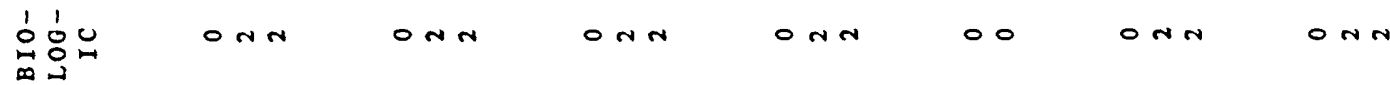

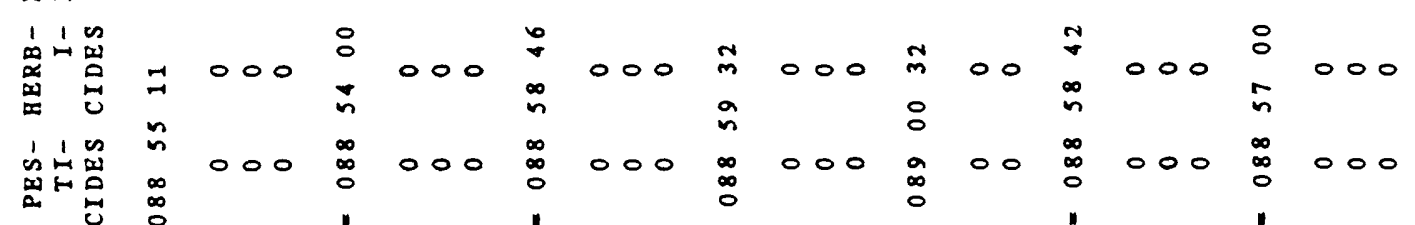

象

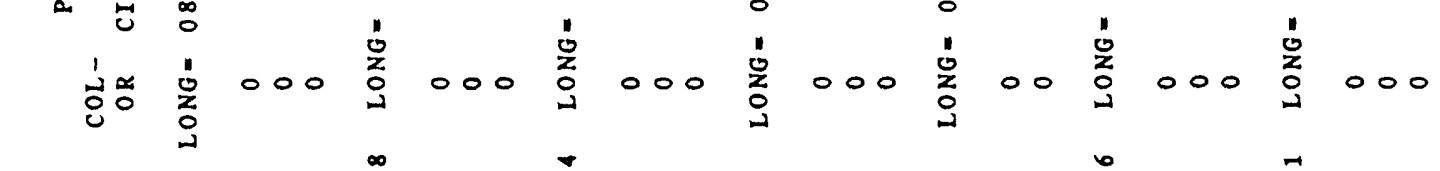

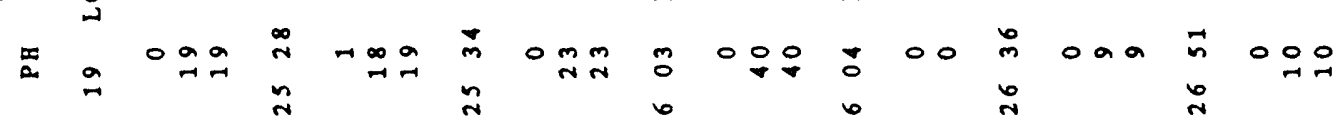

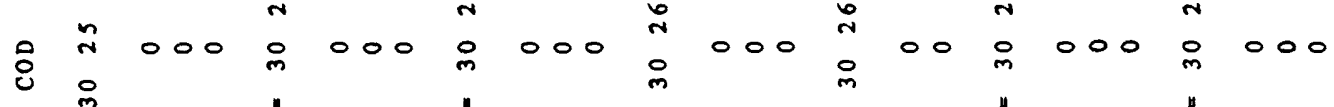
定 点落

\section{naำ} -

旨兵点

000

电

事 t 


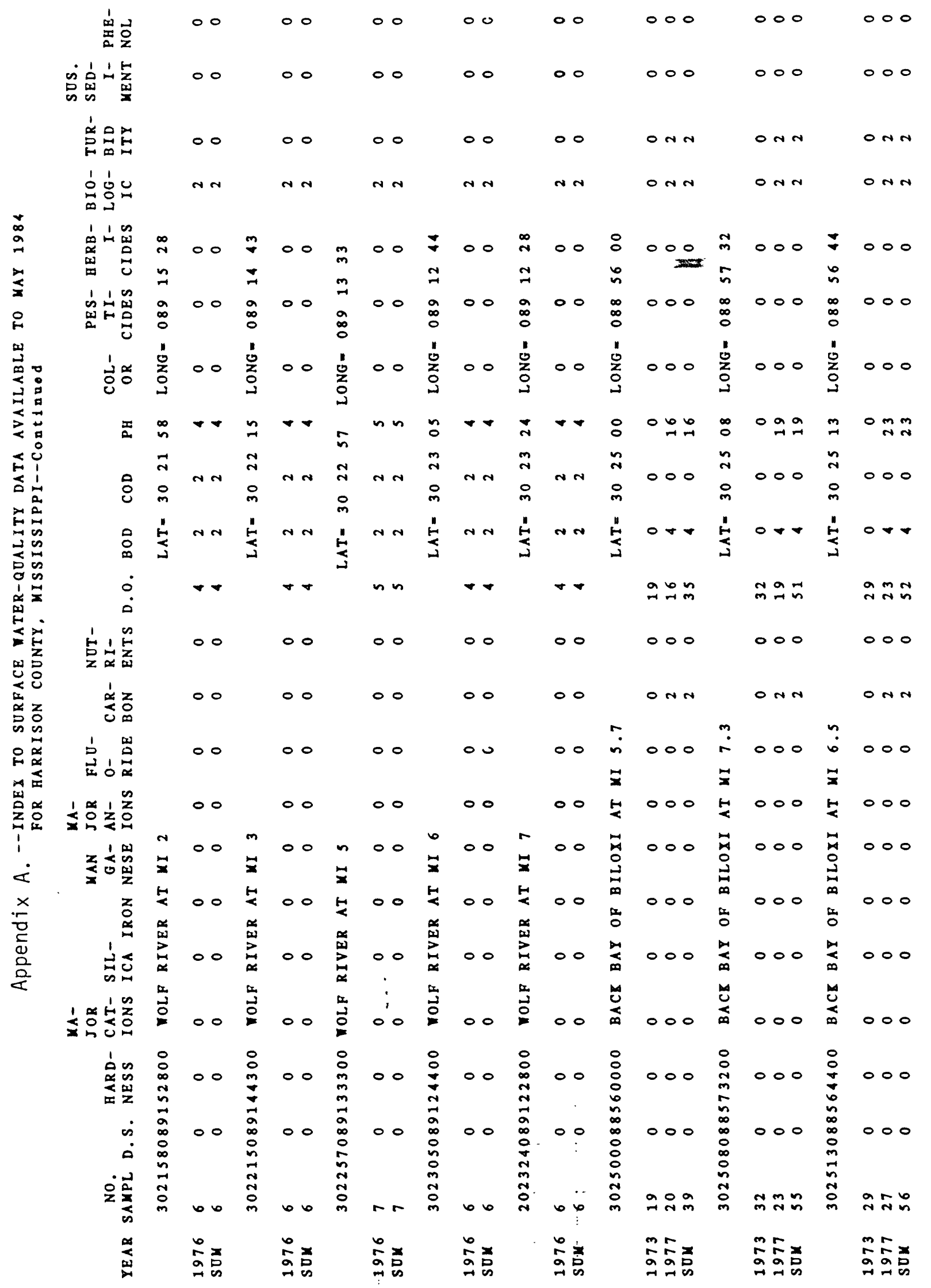




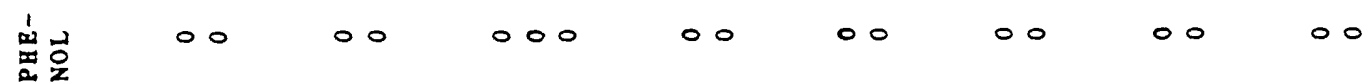

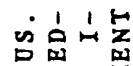

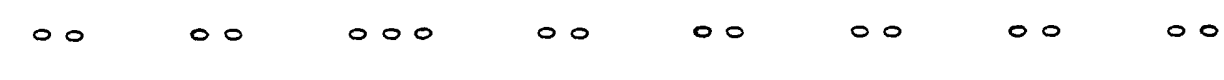

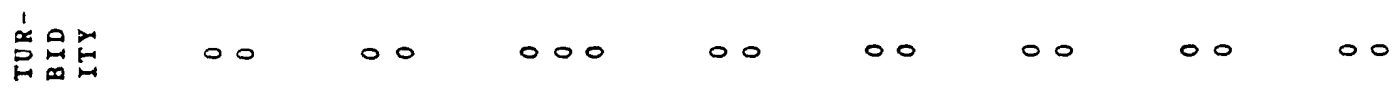

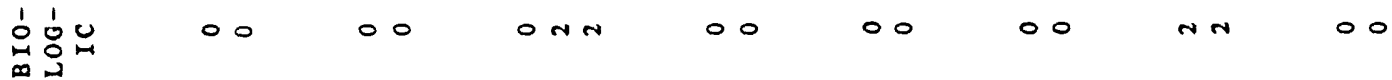

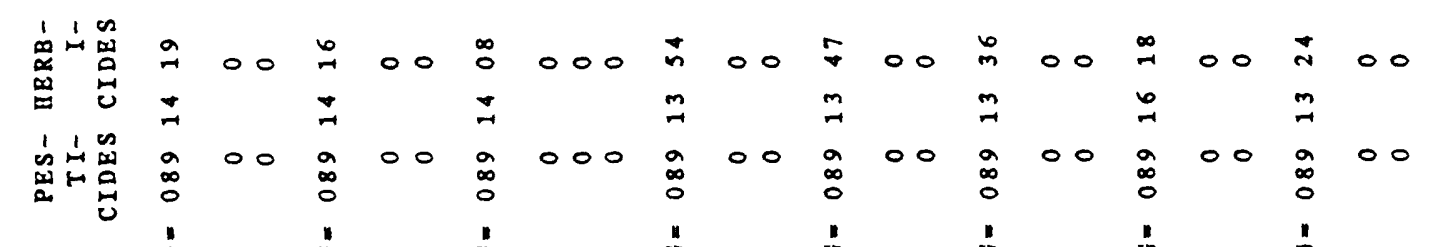

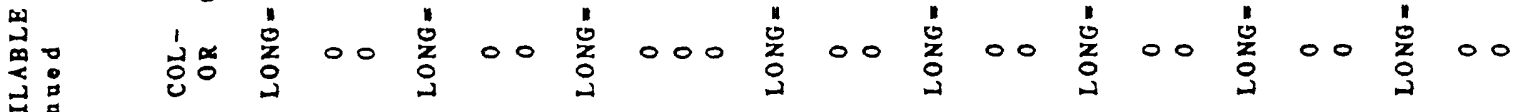

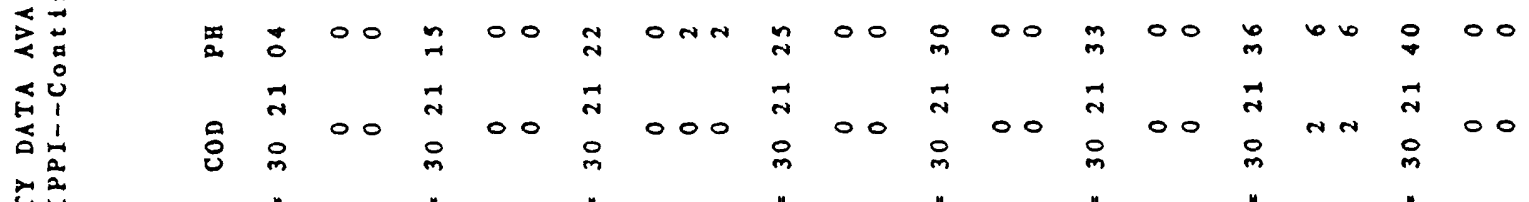
鸬蕉 告 $\dot{0}$

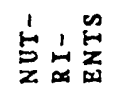

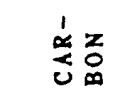
$00 \quad 00$ 方黄
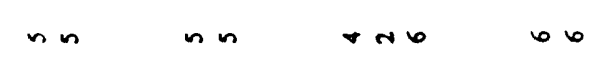

000

000

00

000

0000

$\circ \circ$

$00 \quad 00$ × $z$

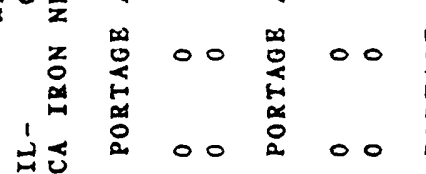
1乎它出

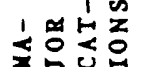

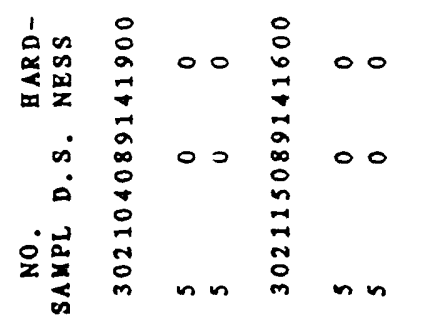

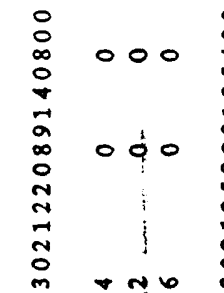

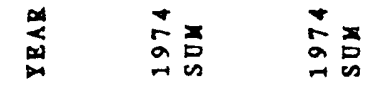

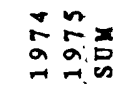

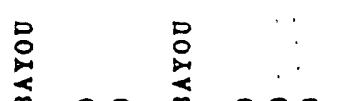

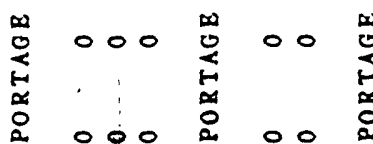




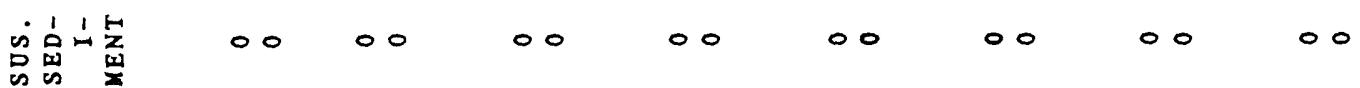

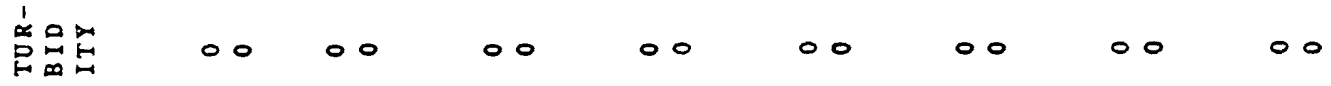

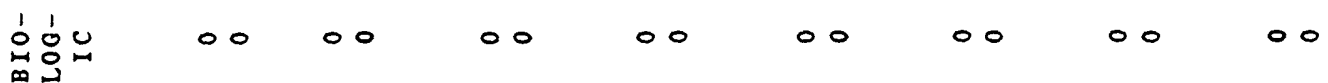

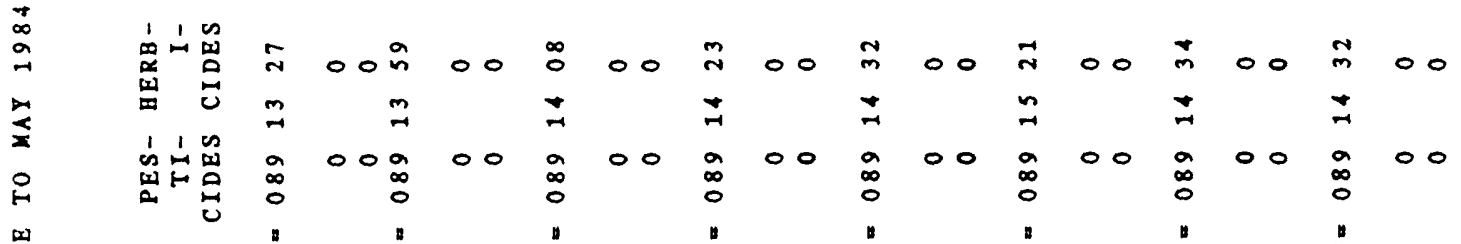

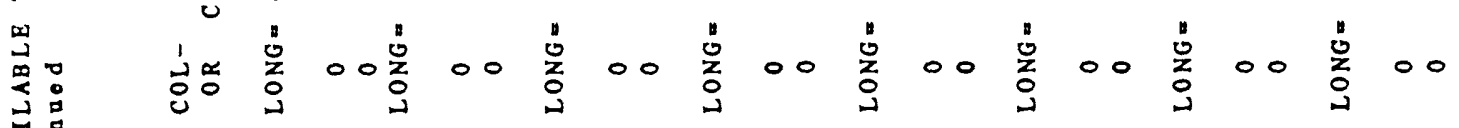

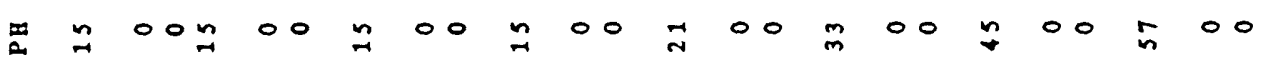

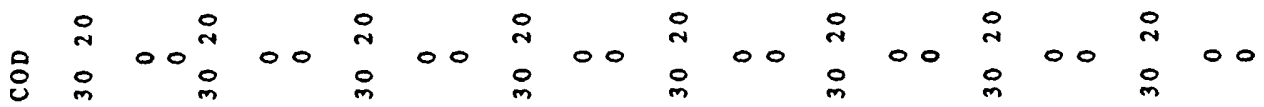
○

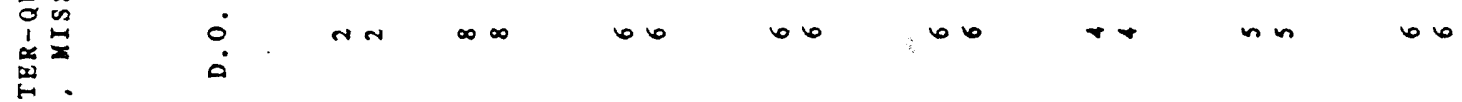

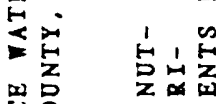

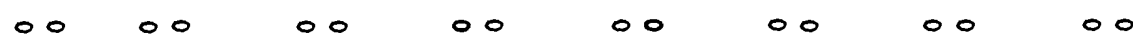

율

닐

$1 \frac{1}{1} \div$

我交苔

z发留

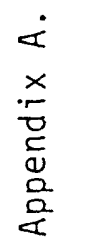

$$
x z
$$

$$
0000
$$

9 \&

$\pm 00_{H}$.

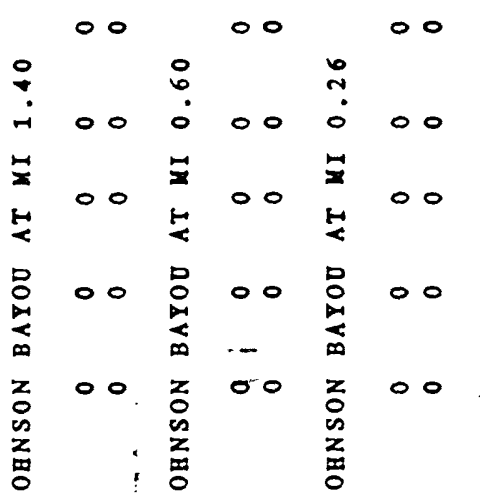

1 영르을

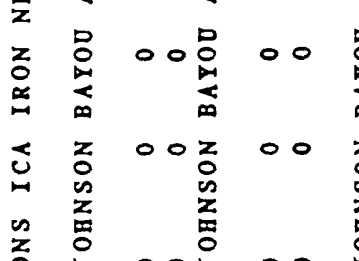

象全

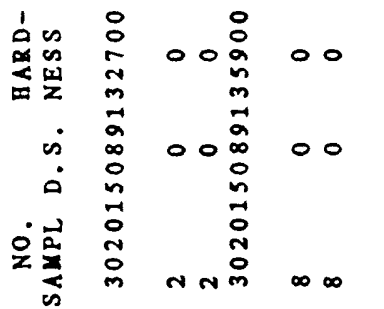

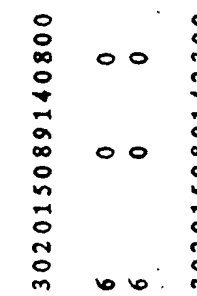

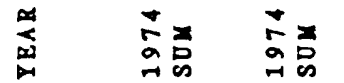

$+$

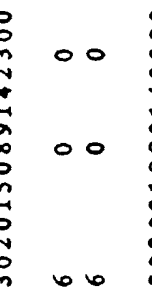

s.

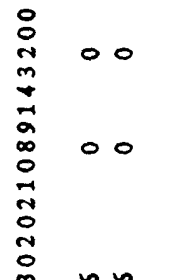

妾竞.

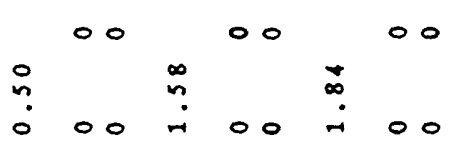

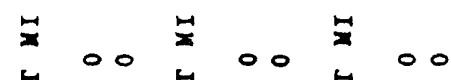

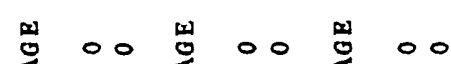

स

\& $00 \% 00 \% 00$

吕 品 品

00

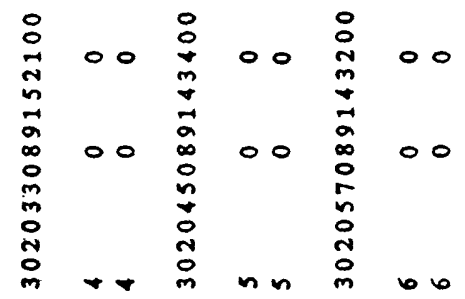

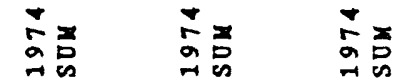




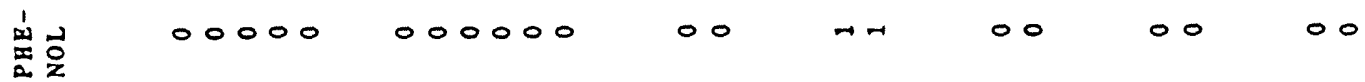

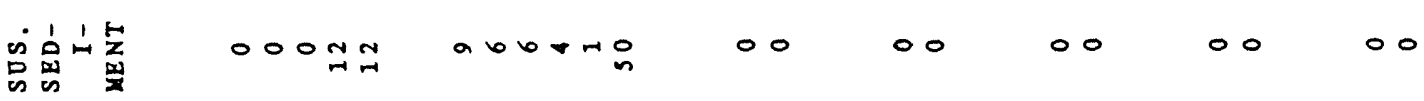

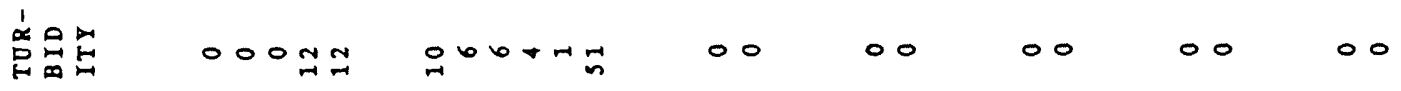

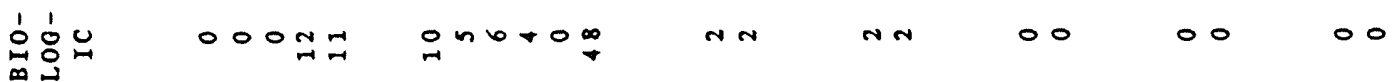
d

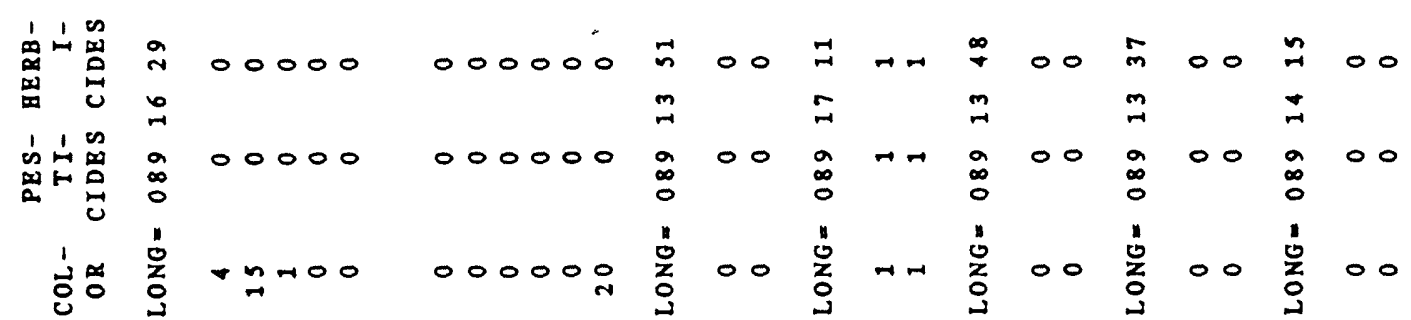
D 0 M

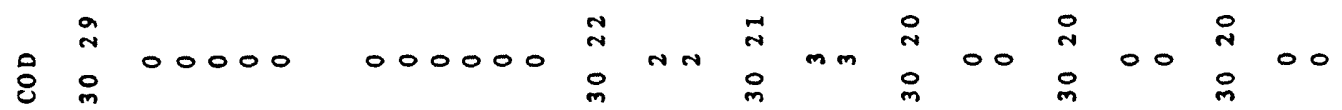

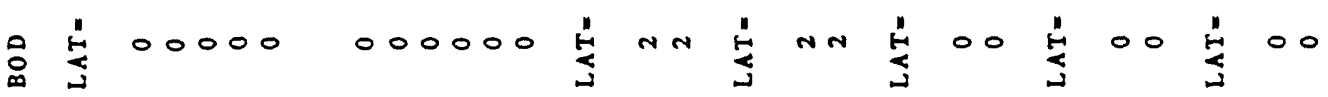

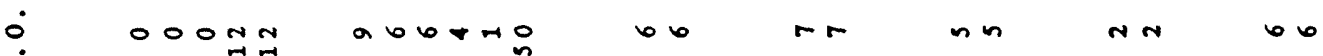
崖管

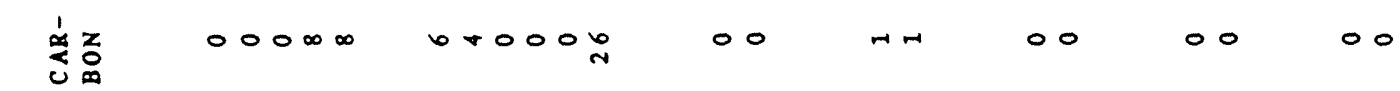

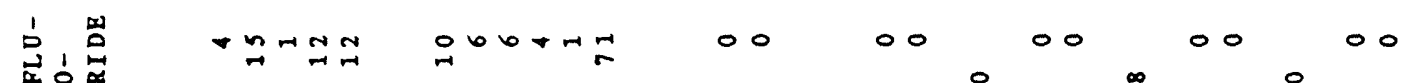

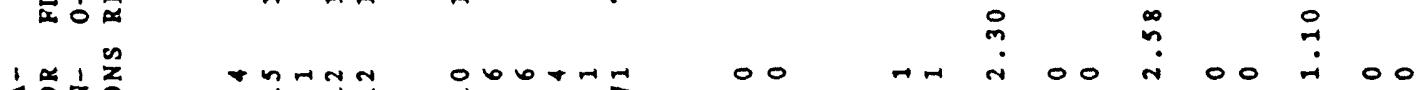

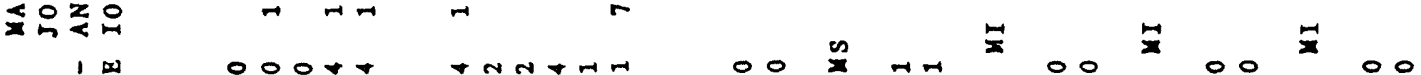

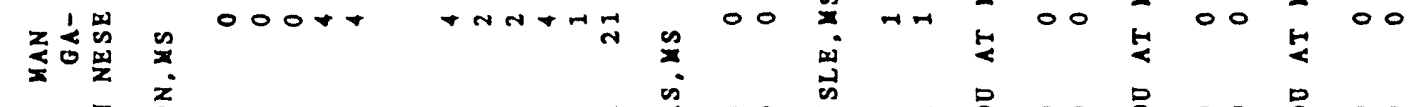
zo $\stackrel{1}{1} 0$

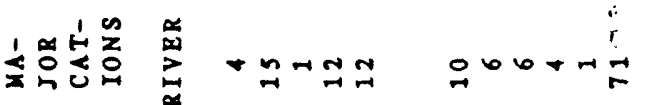

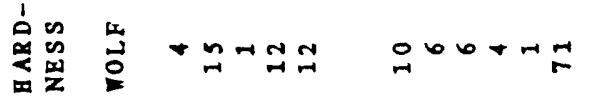

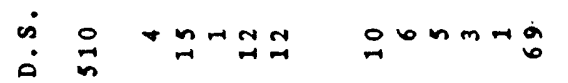

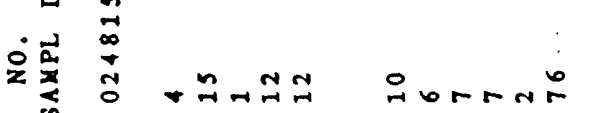

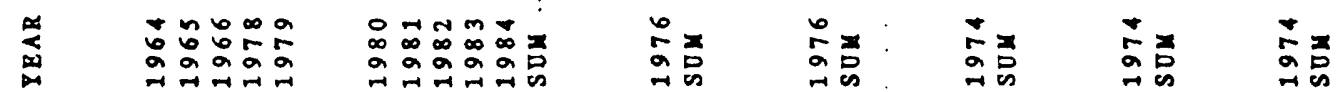

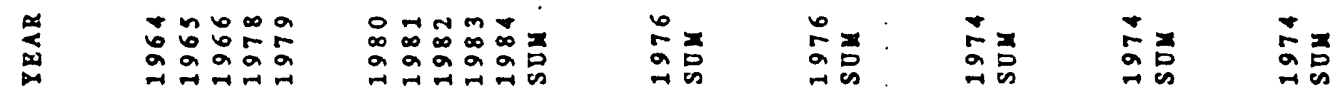

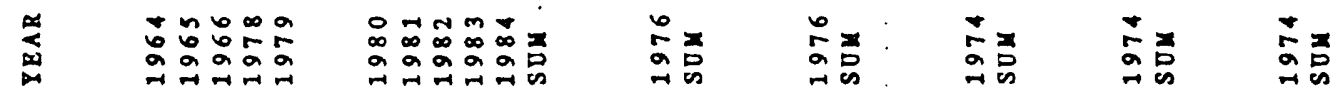

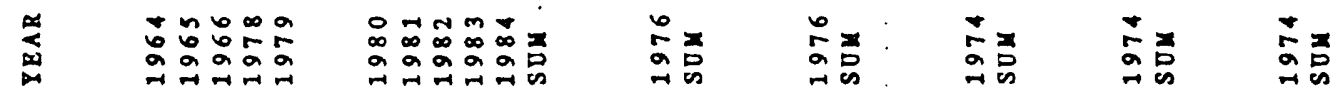
$\begin{array}{lll}0 & 0 \\ 0 & 0 & 0 \\ 0 & & \end{array}$ 品 00 品 00

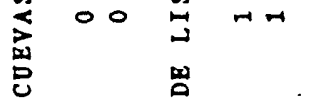

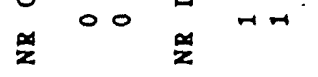

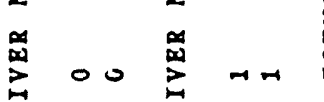

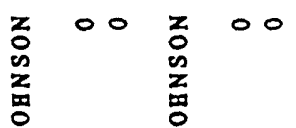
范 $00 \stackrel{0}{0} H$ 0
0
$\infty$
+
$m$
0
0
$\infty$
0
$\vdots$
0
0

0

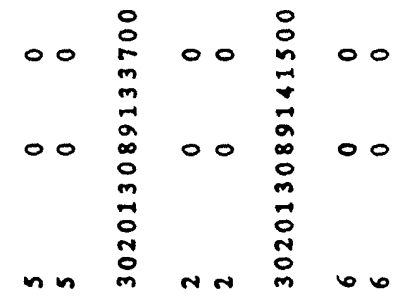




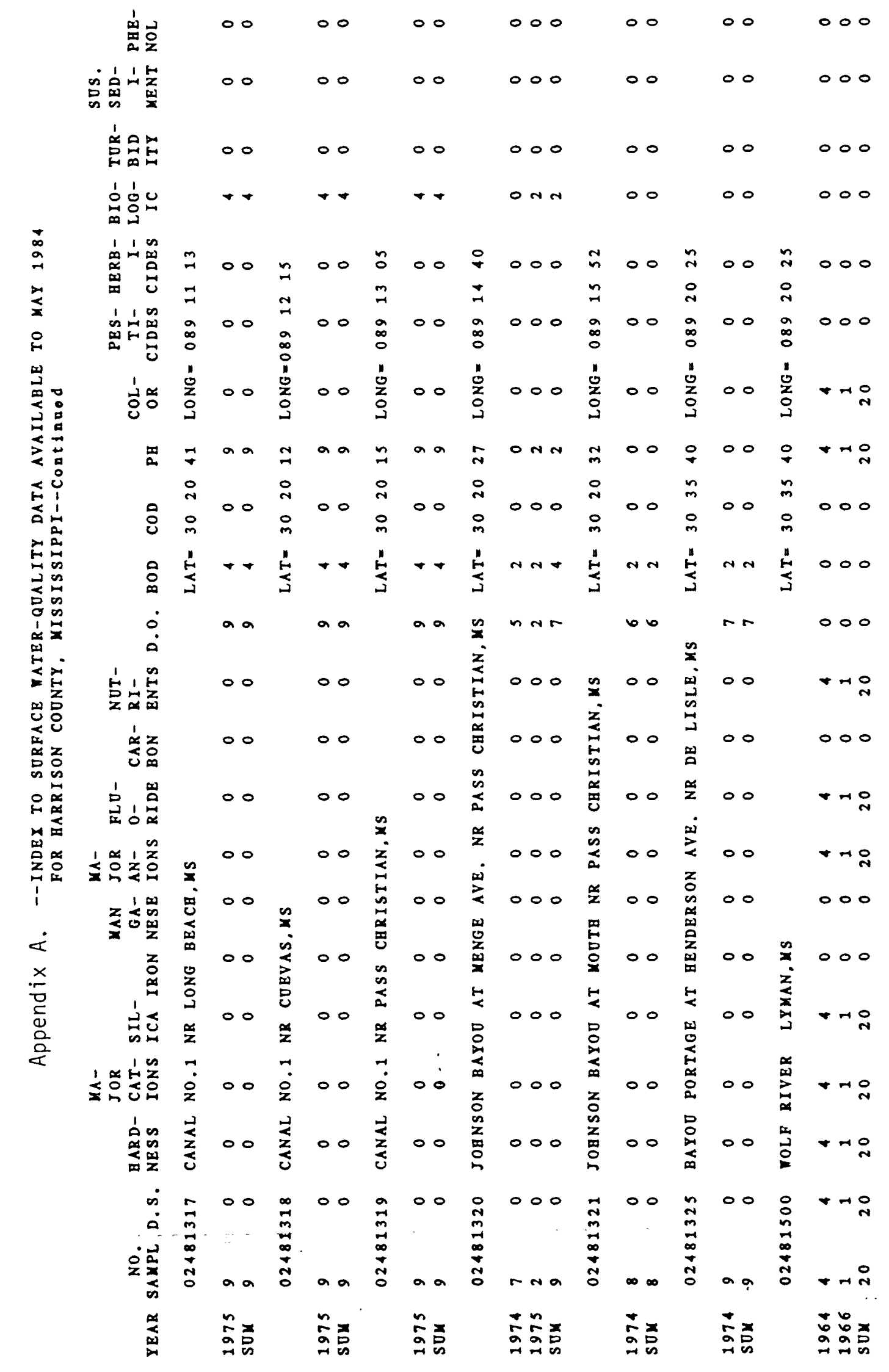




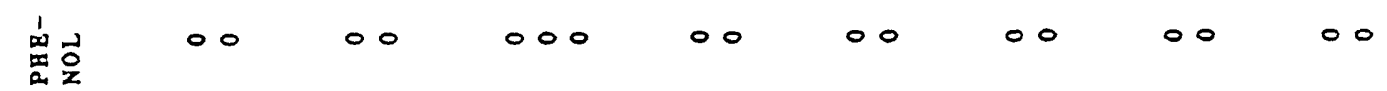

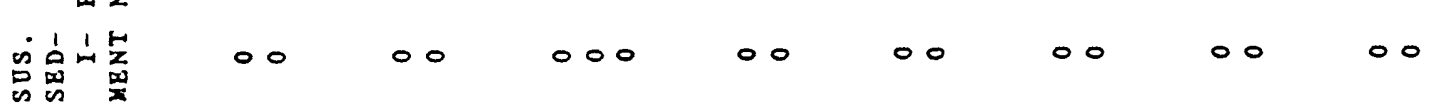

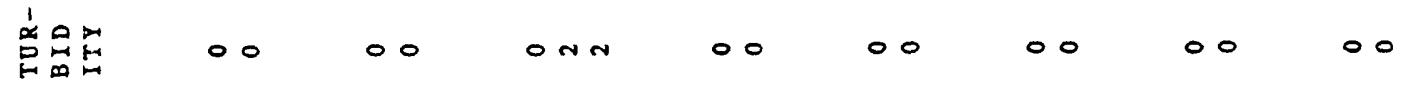

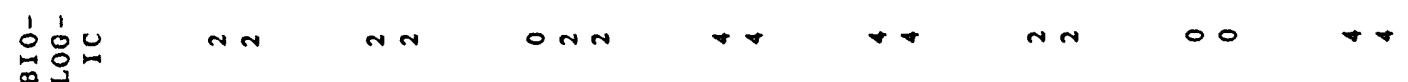

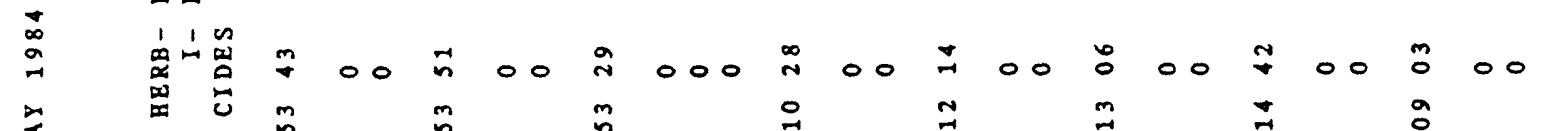

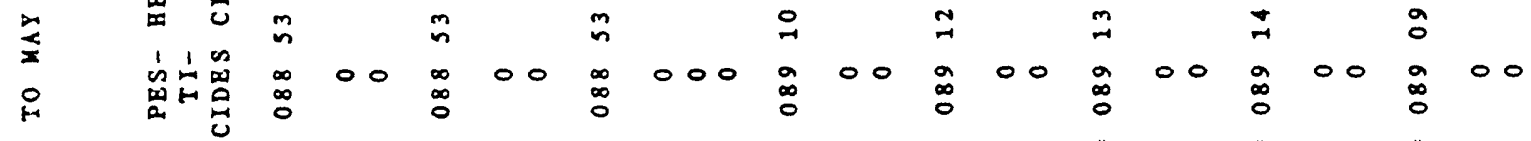

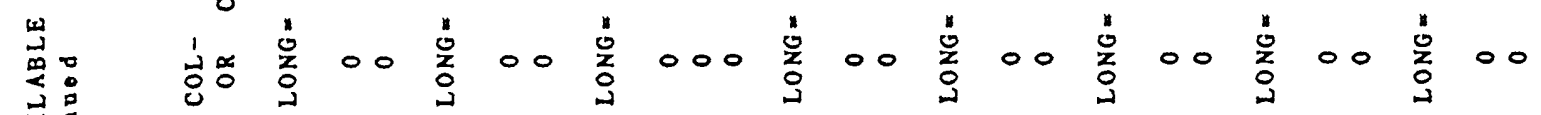

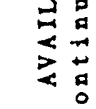

飞它

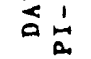
En 表出 on

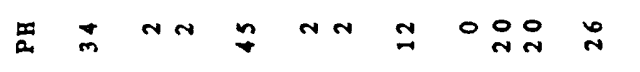

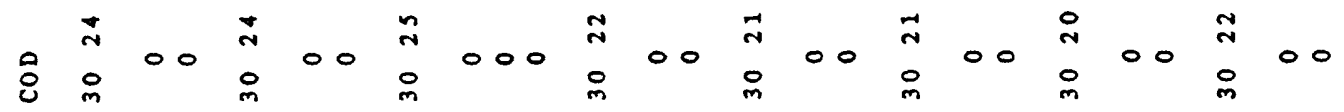

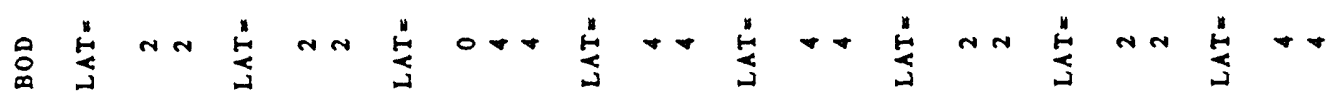

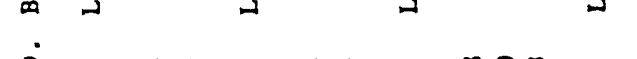

点至策

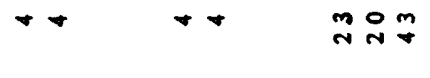

a $a$

a a

$n n$

in $n$

a a

受z

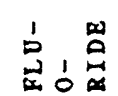

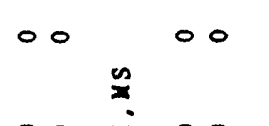

000

$\circ \circ$

$\circ 0$

00

00

00

$1 \times 1, \frac{\infty}{z}$

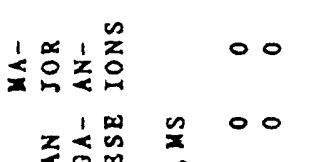

$\sum_{x} 0$

z

岕句

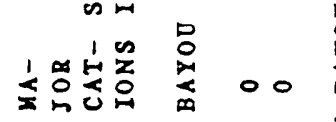

$00 \stackrel{4}{a} 00$

$04 n$

$\circ \circ$

00

00000

0000

$\underset{+}{2}$

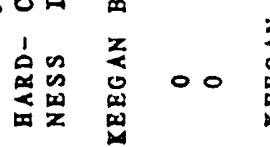

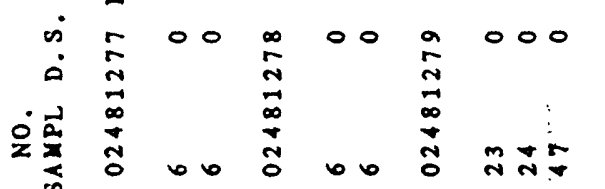

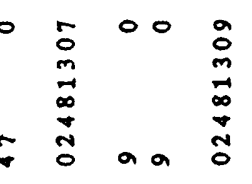

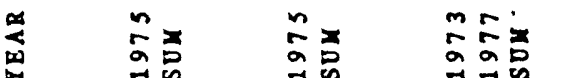

0000

4 


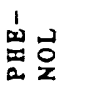

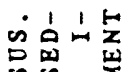

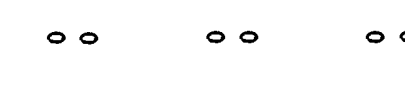

0000

or Hom

○

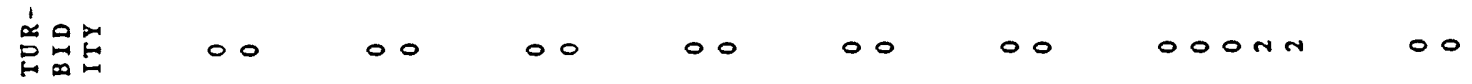
o

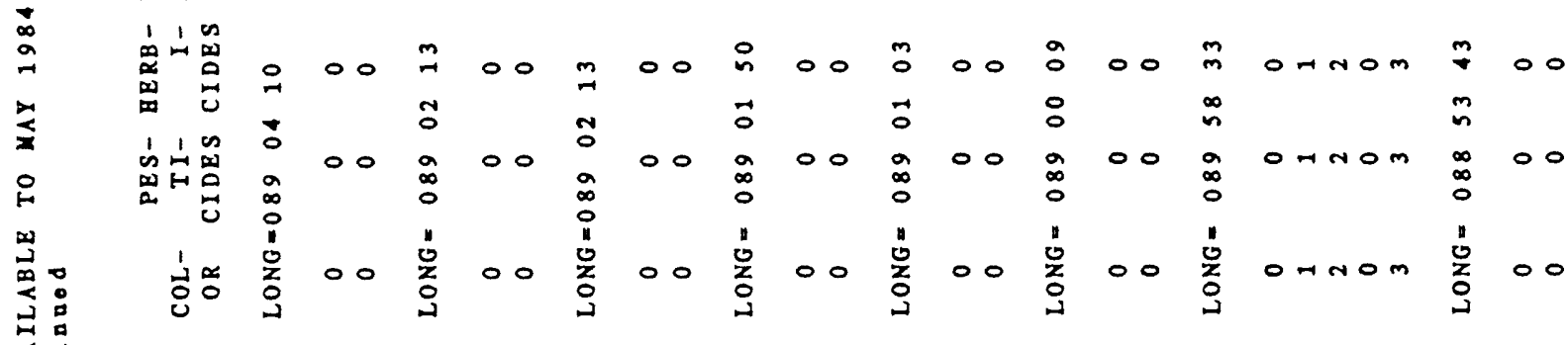

D

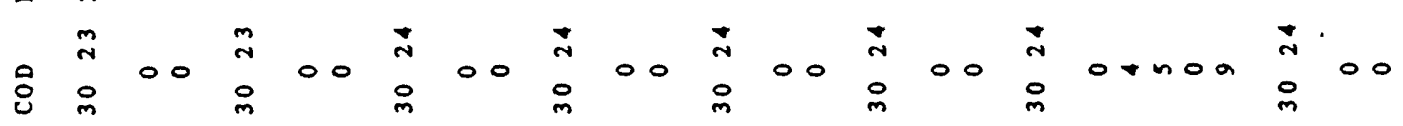

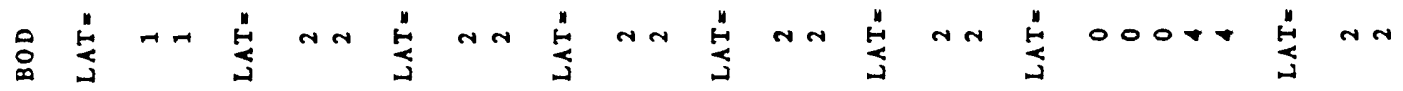

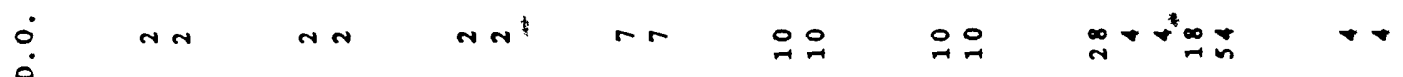
$\leqq \frac{1}{a}$

空空

安躃

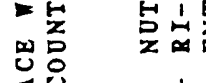

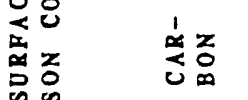

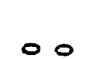

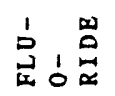

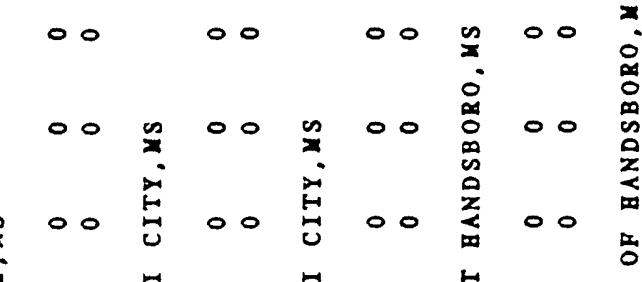

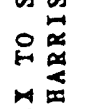

농

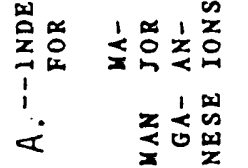

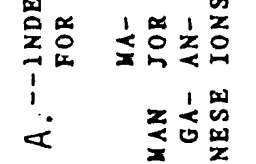

$x$
$: \frac{1}{0}$
$\frac{1}{0}$
$\frac{0}{2}$
$\frac{0}{1}$

总总

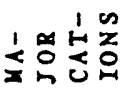

兵舟

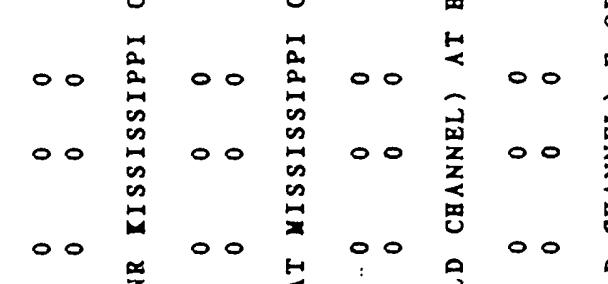

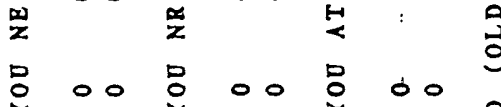

oo

क

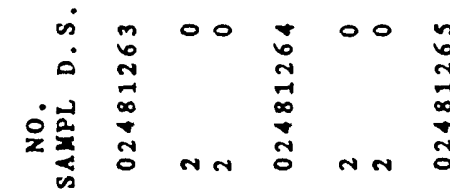

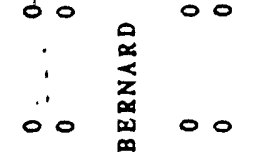

$\begin{array}{lll}1 & 0 & 0 \\ 0 & 0 & 0\end{array}$

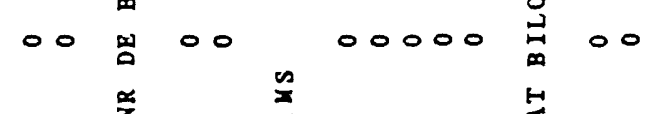

$00 \quad 00000$

$\circ 0$

is 00 ONnn

$\stackrel{2}{*}$

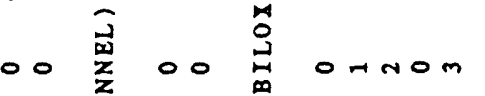

: 00

00

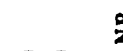

0

00

00

$\pi_{0}^{2}$

$00 \sum$ OnOM

00

o $\stackrel{0}{0} 00$

00

00

OTnOm

怼

$\operatorname{con} 2 \pi$

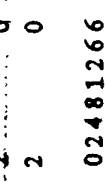

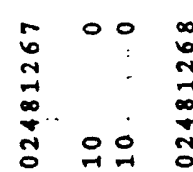

00

$0-n 00$

600

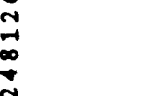

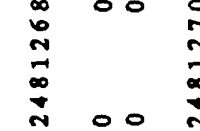

客希

紊总

\section{s.}

管

竞竞

s.

ระ⿰彳

an 


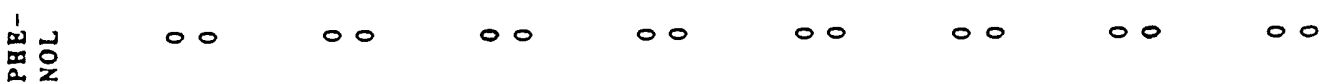

究占定

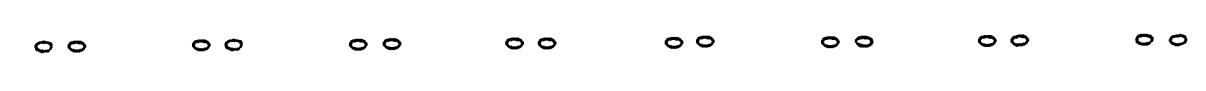

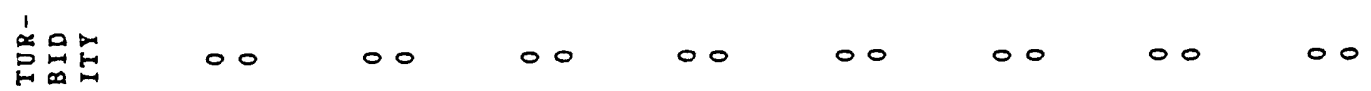

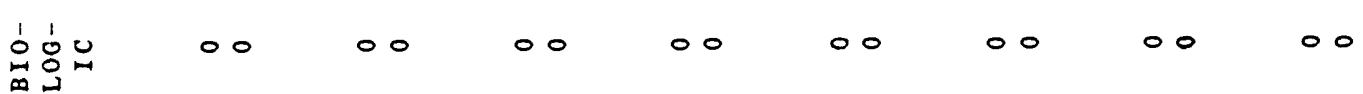

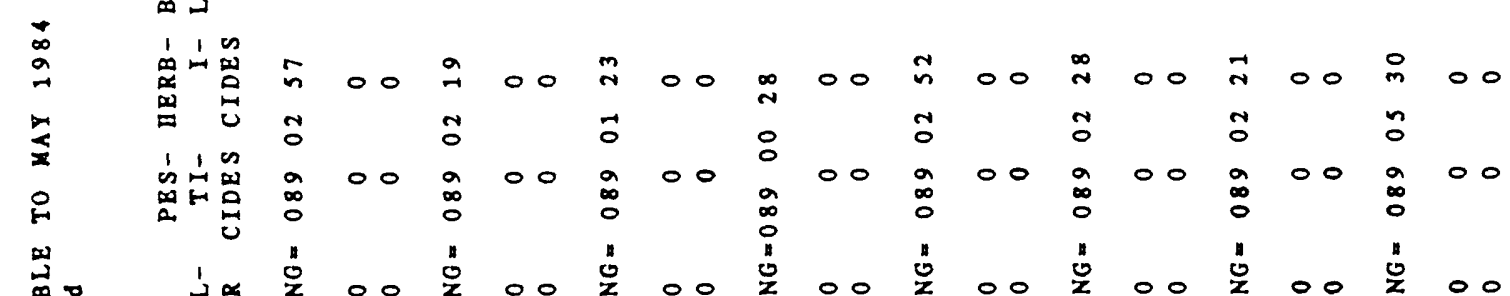

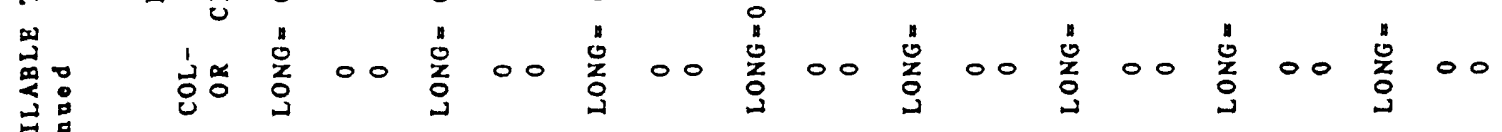
$\sum_{0}$

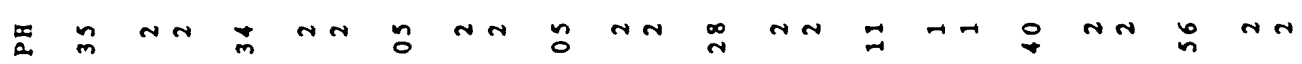

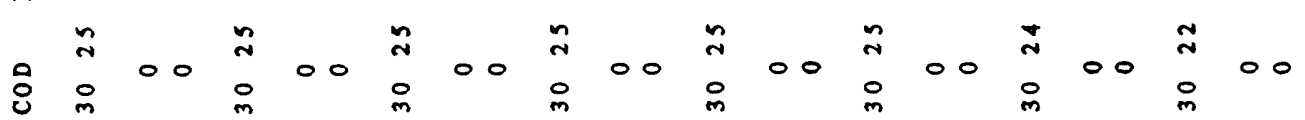
品

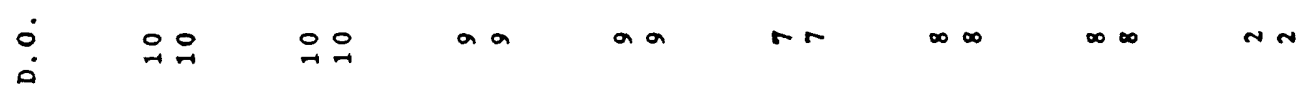
焉

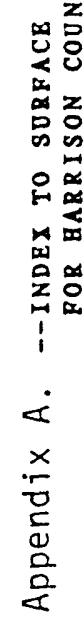

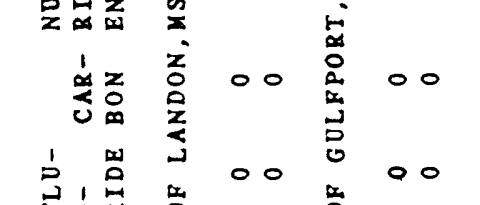
$1,1 z$ in 000 告 00

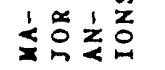
$z \frac{1}{n}$

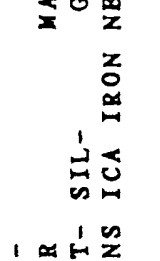

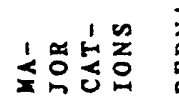

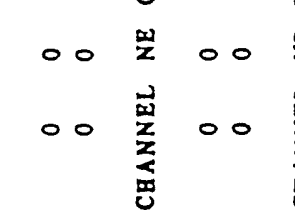

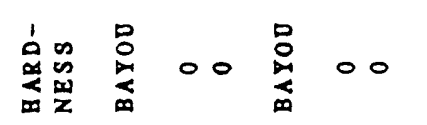

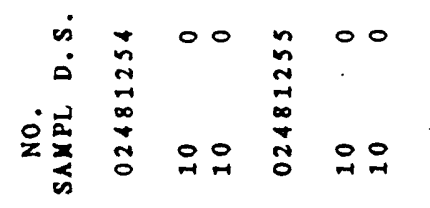

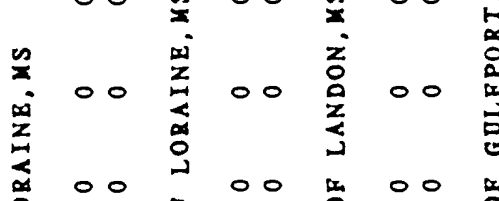
00 00 


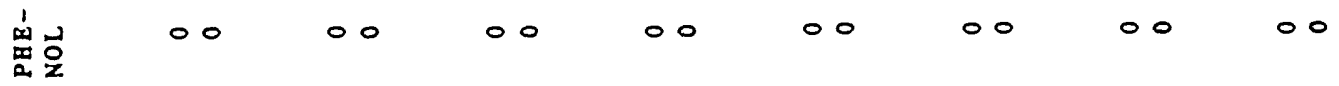

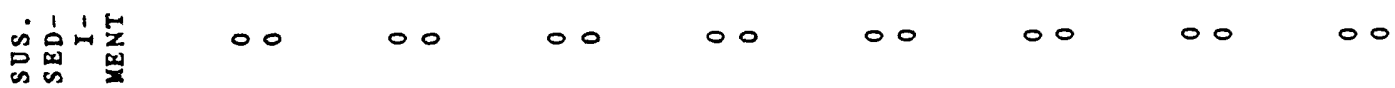

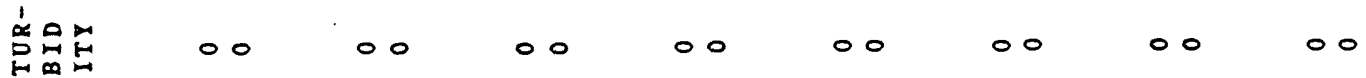

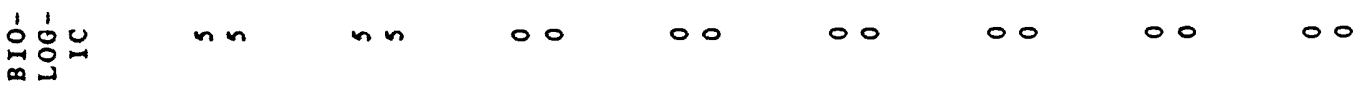

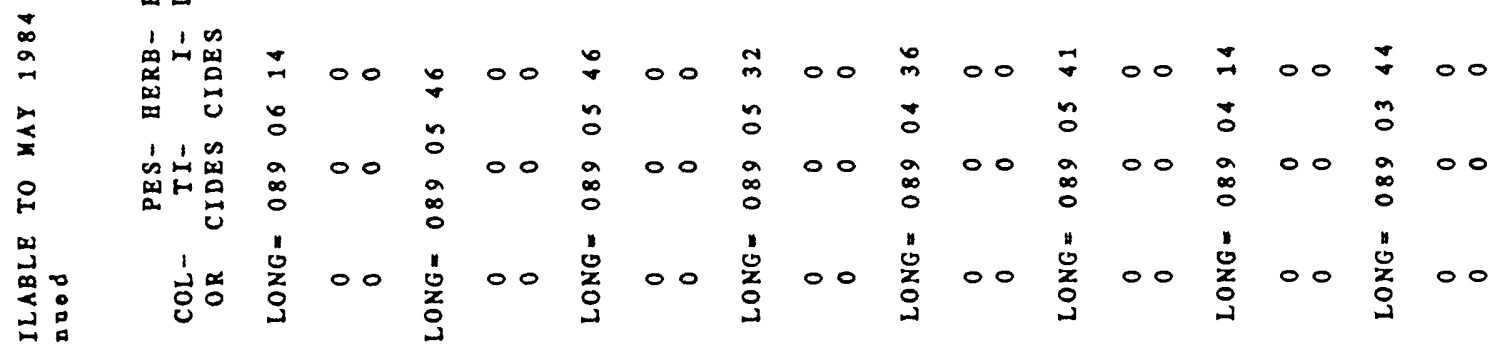
蕰 罗

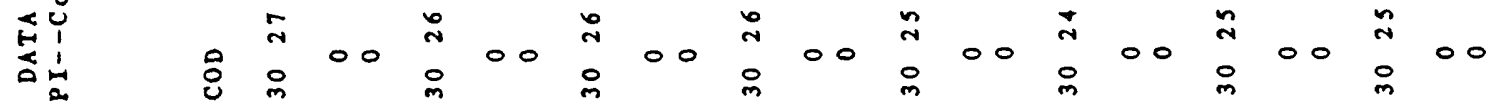

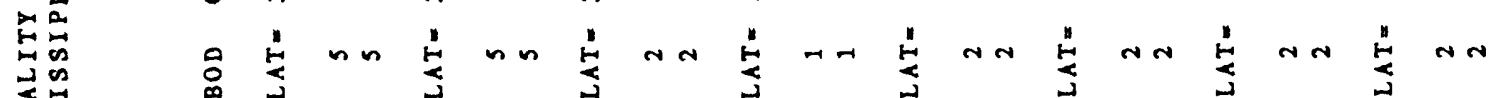
要

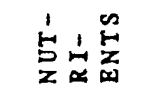<smiles>C1CCCCC1</smiles>

$$
a a
$$

44

方z

$\stackrel{1}{3}$

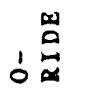

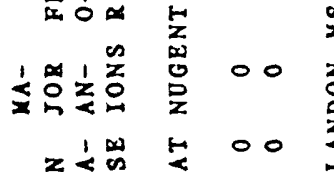

赵倦

$$
00 \quad 0000
$$$$
m m \quad \infty \infty
$$

n

4n

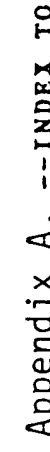

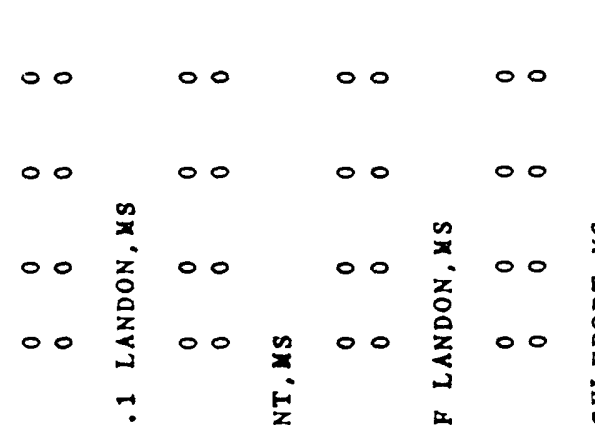$$
\text { 育五 }
$$

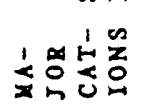

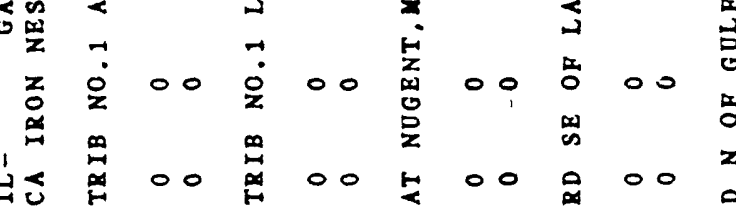

$$
\begin{aligned}
& \text { ए } 00 \text { o } 00
\end{aligned}
$$

范

范

它我

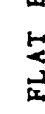

$00 \sum_{\frac{1}{5}}^{\infty} 00$

t) 00

is

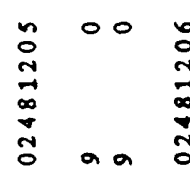

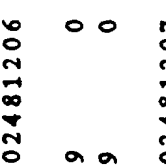

$\varliminf_{\substack{\infty\\}}^{\infty}$

$\stackrel{0}{\leftarrow a}$

$\stackrel{0}{a}$

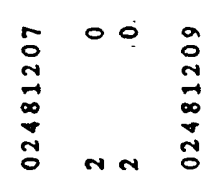

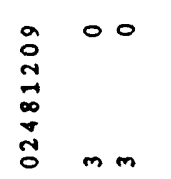

衣合
旁言
00

00

00

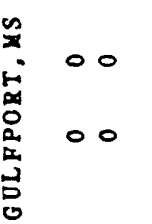

00

z 00

昰 00

: 00

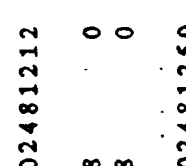

00

00 豆

00000 00 म 00 मू 00 o 0 o 0 足 00

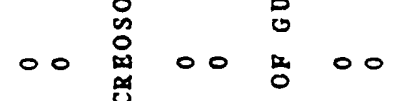
$00 \% 00^{\frac{\infty}{z}} 00$ 幽 $000 \stackrel{\substack{\infty \\ \infty}}{\infty} 00$ 幽 紫

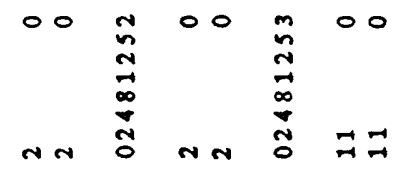

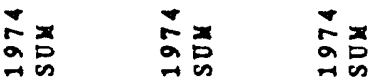




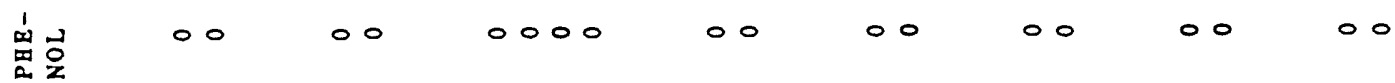

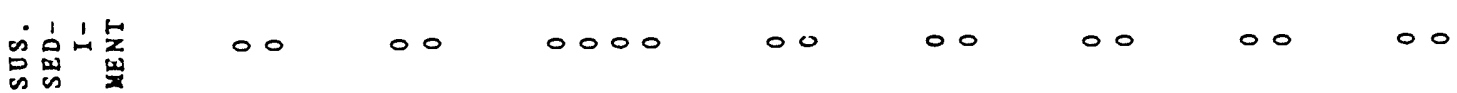

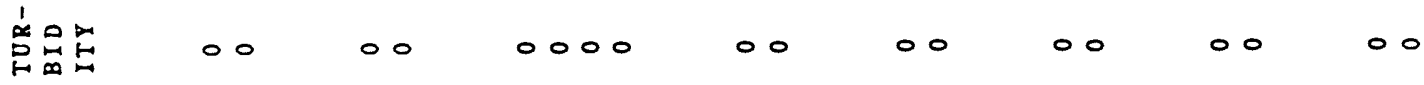

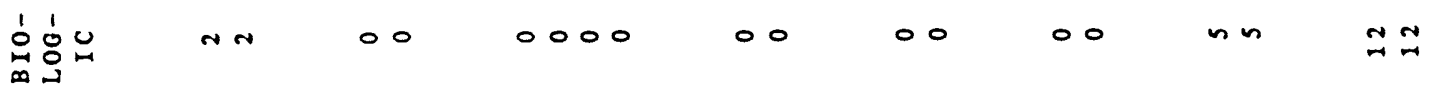

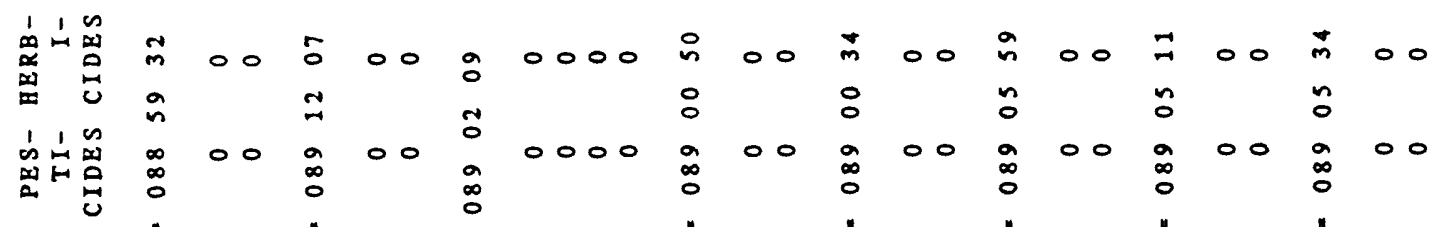

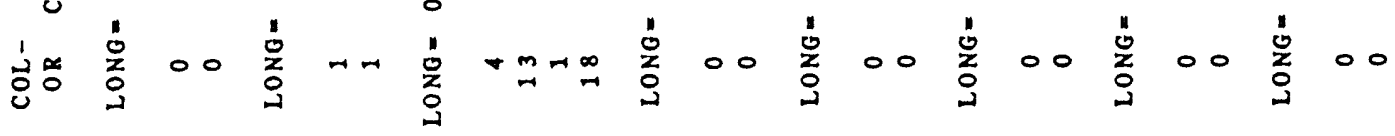

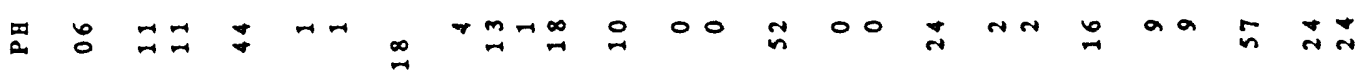

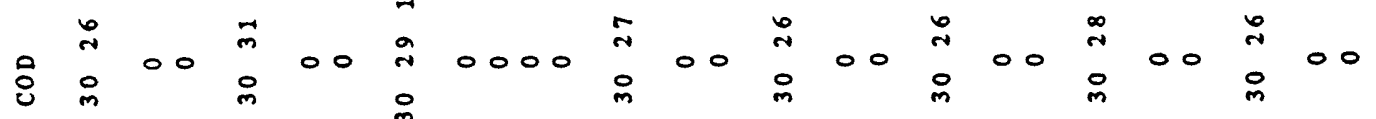
品苞 NN

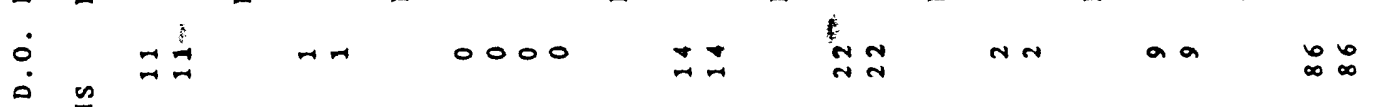

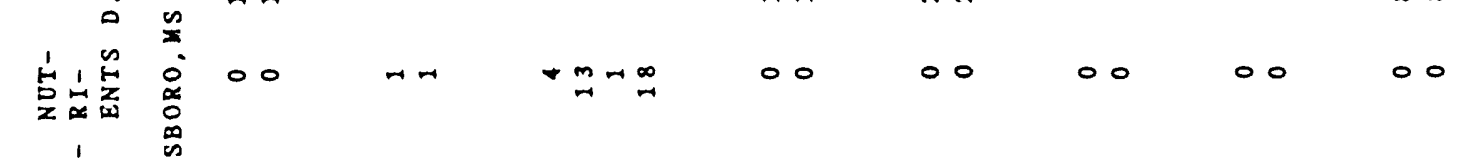

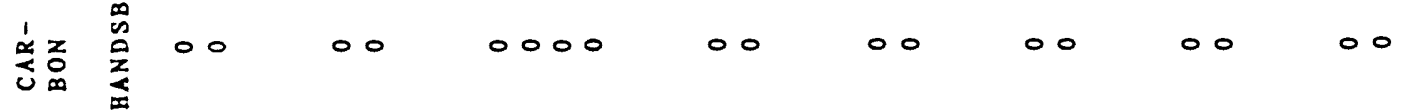

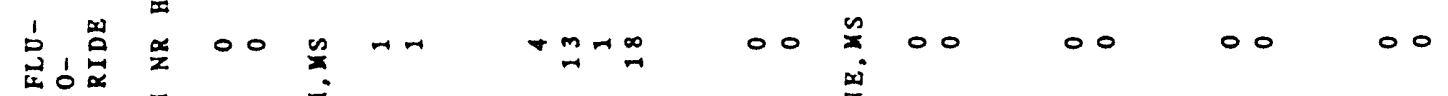

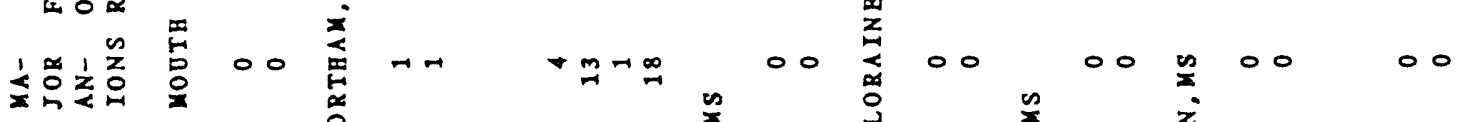

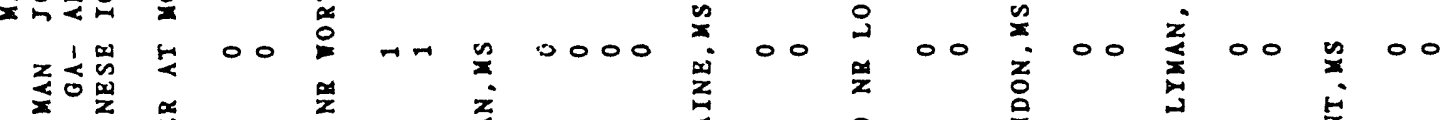

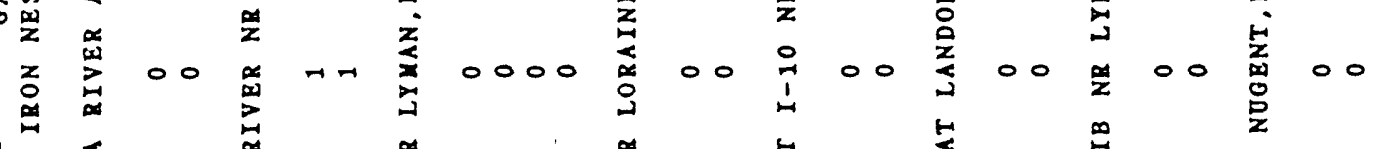

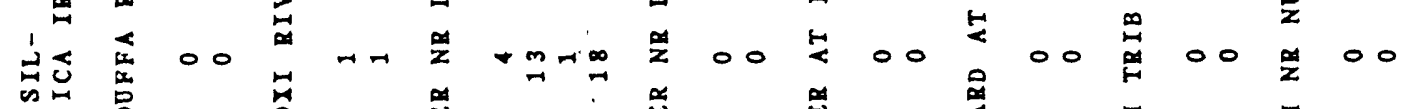

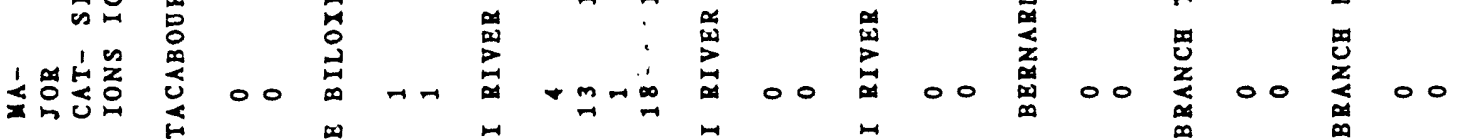
綿出

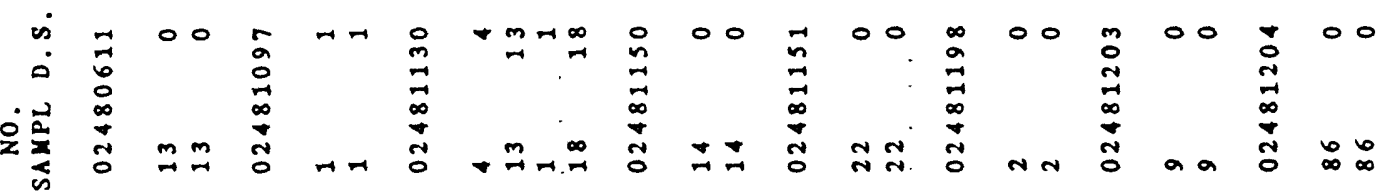

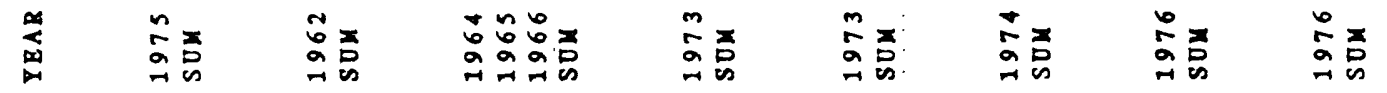




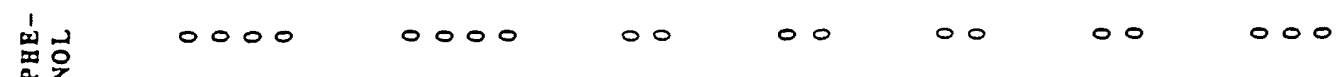

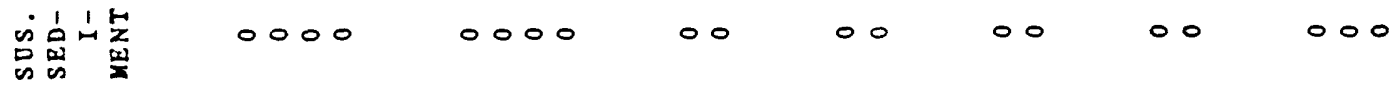

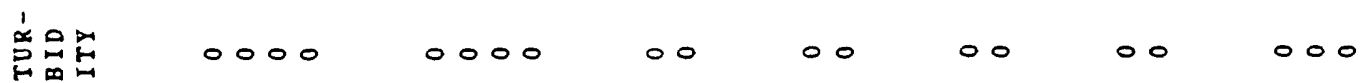

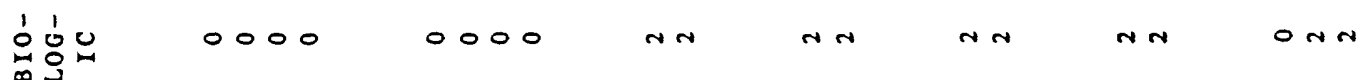

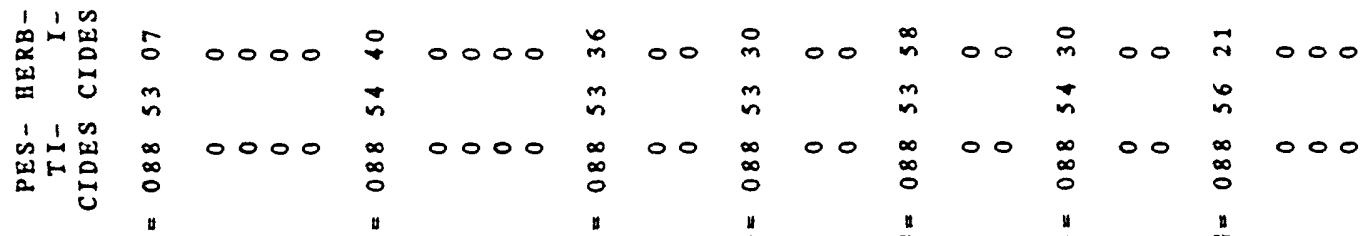
苔范

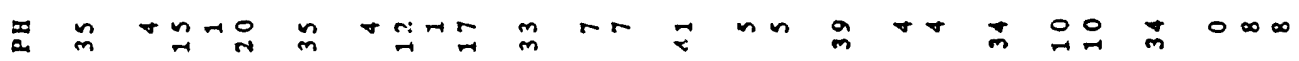

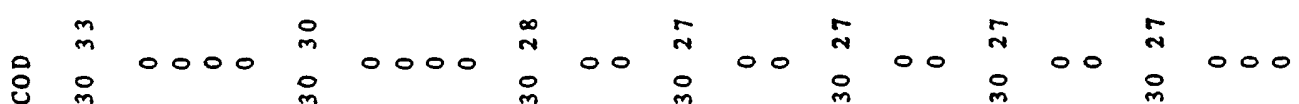
○!

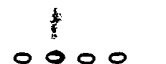
$0000+r$ $n n$

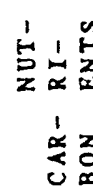
光

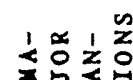

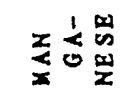
z 武可

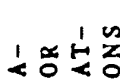

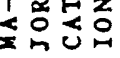

o

$$
120
$$

$$
00
$$

บุ

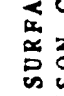

용

$$
\text { is }
$$

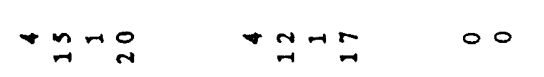

$z$

$$
0000
$$

rogo $\pm 00$

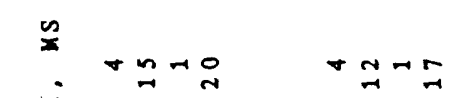

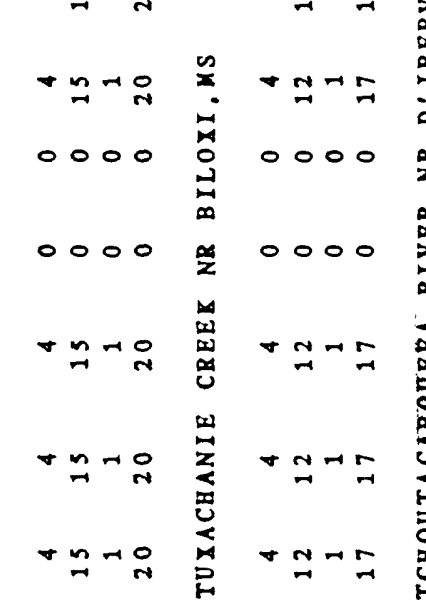

00

00

$00 \stackrel{0}{0} 00 \stackrel{0}{0}$

×

00

00

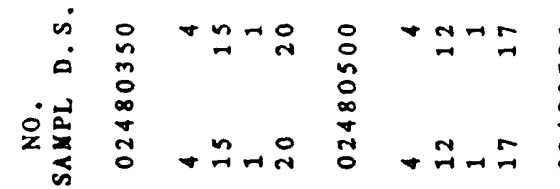

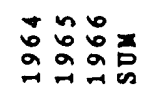

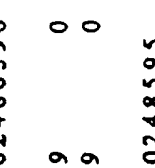

葛

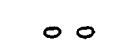

00

00

00

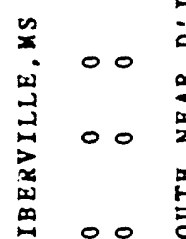

z 00

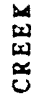

00

$00 \stackrel{n}{x} 00$

00

00 율 00

$00 \% 00$

00

$\sum_{\infty}^{\infty} 00$

00

近 00

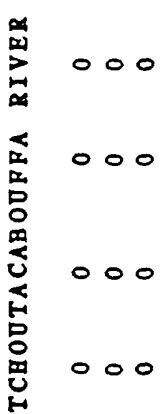

00

0 in 00

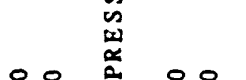

00

0

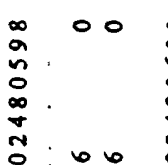

$\begin{array}{llll}0 & 0 & 0 & n \\ 0 & & 0 \\ 0 & & 0 \\ 0 & & & 0 \\ 0 & 0 & & \\ 0 & 7 & N & N\end{array}$

000

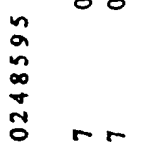

$\stackrel{n}{n}$

$n \geq$

$\ln _{\substack{n \\ 0}}^{n}$

군ㄷN 
綮 穴命占点 品的 致

1

䍃虽齿

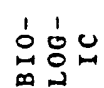

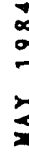

\section{0}

年哇

की 10

悉是㫕

幽 잉 ํㅇㅇ용

要

ठำ

응

$\dot{0}$

皆点点

孚

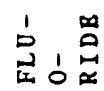

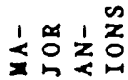

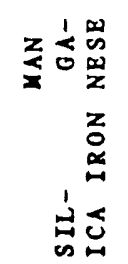

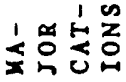

点品

is

家最

离

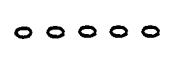

00000

00000

00000

000

i 0000

in

m

$\infty_{\infty}^{\infty} 00000$

"I

nhano

$n n \pi m 0$

00000

00000

r.

$00000 \quad 0-1$

nhano

006

00000

000

$\cos +1+0$

10

$\operatorname{tan-t}$

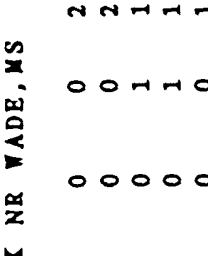

$44 h \rightarrow 0$

ณ $4 n \pi \mu$

c) $4-1-1$

吕

Com

nhm-

$$
\begin{array}{cc}
0 \\
0 \\
\vdots \\
\vdots \\
\vdots \\
\vdots \\
\vdots
\end{array}
$$

4hand

$\infty a 0=7$

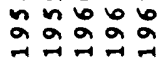

000

O-r

$000 \%$

000

00000

00000

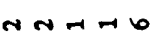

00000

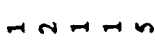

N HO

on

o

000

$+\infty$

;

oon

or

.

$\pi \pi^{4}$

送竞

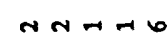

०ศन-m

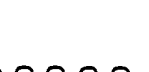

00000

namp

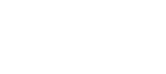

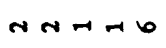

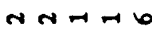

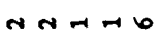

à

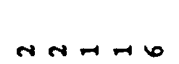

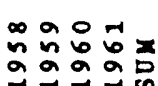

00000

000000

00000

00000

000000

00000

00000000000

00000

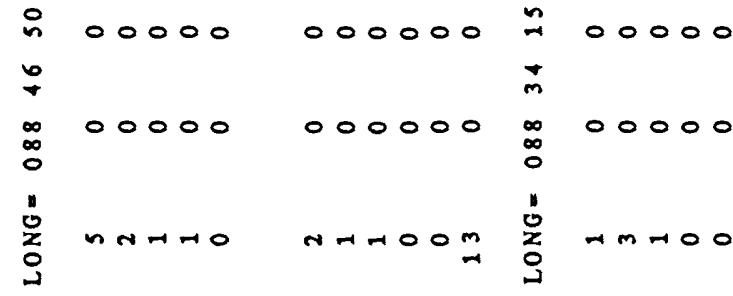

- nntho

masmnt

mmor

00000

000000

00000

$\stackrel{1}{\omega} 00000$

000000

00000

00000

$\operatorname{monman}$

$0000 n$

nAM-O

$m m-00$

00000000000

00000

nhamo

hh000n

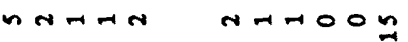

OOHOO

20

nOOOO

$\operatorname{shnhn}$

点

$\operatorname{nnh} n$

nnmao

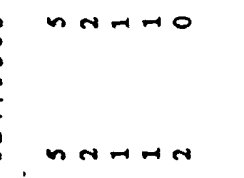

000004

$00000 N$

hrogor

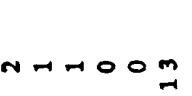

$n-0, \pi$

$\rightarrow m+00$

Hm

$\rightarrow m \rightarrow 00$

AmAnO

$0-000$

00000

mmaro

$1700 \mathrm{~m}$

maamnim

$-m+00$

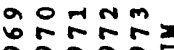

ㅇํำลำ
$A m a n d$

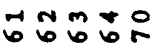

a 006 a 
มี

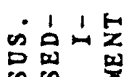

000

00000

00000

竞聶

ํํำ

0000

00

00000

00

00000

00

$0000 n$

11

艒

0000

00

00000

$-1$

onodo

on

o o dnh

$11 \infty$

番思罟

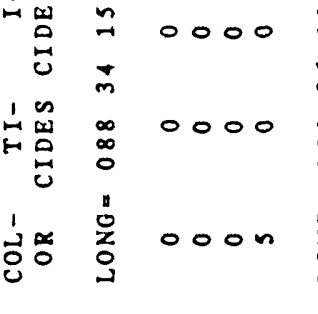

00000000

$00 \% 0000$

0

$00000 \%$

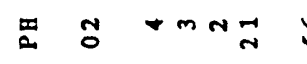

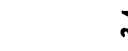

$00 \quad \begin{aligned} & \infty \\ & \infty \\ & 0\end{aligned}$

$m$

$00 \bigsqcup_{0}^{1} 0_{1}^{1} 00000$

$\begin{array}{lll}0 & \infty \\ 0 & \infty \\ 0\end{array}$

00000

00

00000

¿ ${ }_{m}^{n} 0000$

00 에 $0600 \infty$

00

ond 000

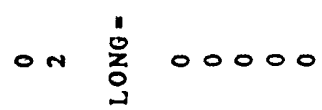

$0+50 m m$

$m \underbrace{\infty}$

$00 m 6 n$

$00 \stackrel{a}{n} 00000$

00

$0 \stackrel{1}{1} 0000$

00

$\dot{0}$

$\stackrel{1}{H} \underset{z}{1} \stackrel{n}{z}$

$\sum_{0}^{1} z_{0}^{2}$

10

至监

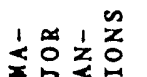

100

$$
\operatorname{rno}
$$

$n n$

$n+0000$

$0-1$

00000

o $0 h$

00000

on $\prod_{n}^{n}$ moont

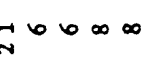

$0 n$

ñmma

$m 0$

ำ

onor an oo 00000

00

00000

$\circ \circ$

00000

0000

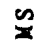

- z $z 0000$

o o z

$00 \$ 30000$

00

$00 \frac{2}{2}$

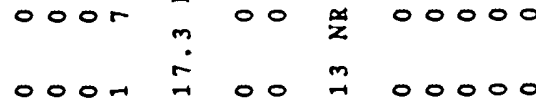

$00 \quad r$

0000

ON

$0000 n$

00000

00000

y

z $\$ 0000$

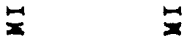

岂氙

001

00000

00

0000

00

- $\infty$

00000

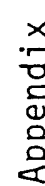

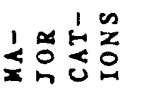

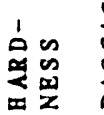

$000 n$

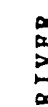

$00 \sum_{n}^{\infty} 00000$

$\circ \circ$

00000

00

00000

00000

i0000

00

0000

政

00000

00 u 00000

(

00000

00000

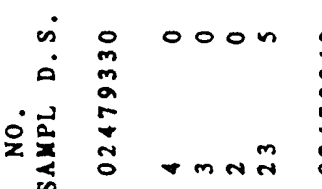

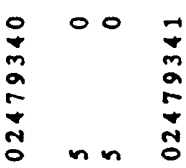

00000

00

$n$
$\vdots$
$a$
$\vdots$
$\vdots$
0

0

00000

ก 0000

-

Nnm $n$

$m n$

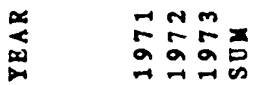

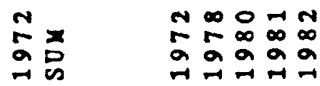

咅咅

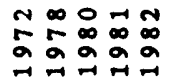

$\mathfrak{m}_{\substack{\infty \\ \infty}}^{m}$

$m n m a$

$20 \% 65$

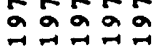




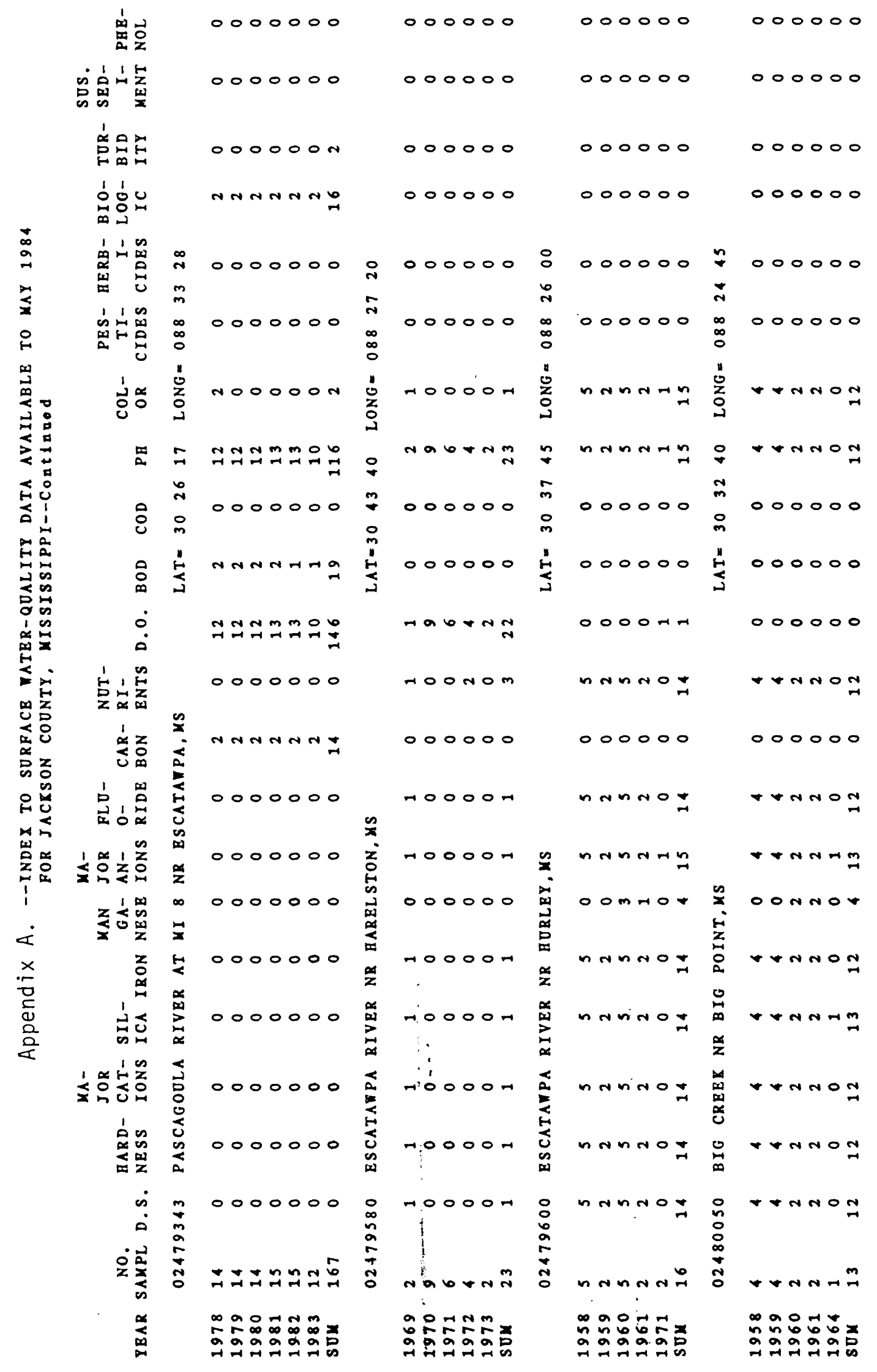


มี

0000000

000

000

0000

0000000

i占古艺

00000

00000

000

0000

00000

$\circ 0$

1.

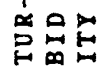

客高

00000

00

000

000

0000

o nom

on

\section{1 in}

00

000

mor

moom

monoo

No

$\approx 00000$ a 00000

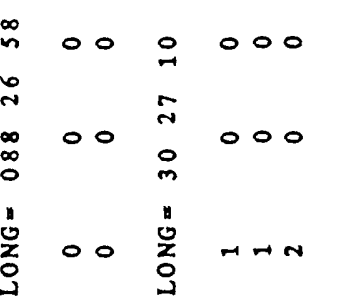

$\sim 000$ i

离

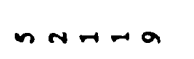

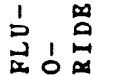

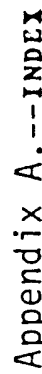

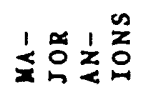

nNATa

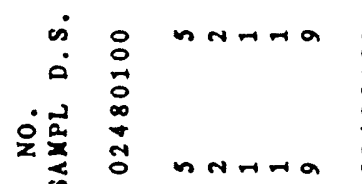

ror$$
\text { ○。 }
$$$$
\text { s00 }
$$$$
000
$$

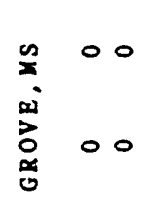

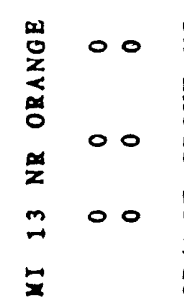

000

000 i

I ror

\section{$\sum_{m}^{\infty} \stackrel{m}{+}$}

000

ror

$$
\text { (2) }
$$

$\rightarrow-\infty$

tio

000

000

$\sum_{\infty}^{\infty}$

a 00 $\xi$ 00

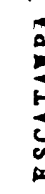

$\rightarrow \rightarrow n$

rom

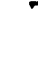

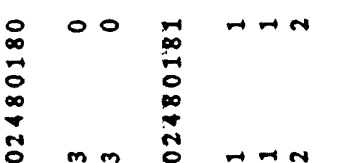

$n$
$\infty$
0
0
$\vdots$
0

$000:$
m.nt.

$\overrightarrow{6} \overrightarrow{2} x$

응ํㅇ 000 $100 e^{2}$

$=$ t

a 000

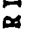

\section{0}

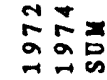

$0000=1$
0000 i
0
0

0000

nnon

0000

00000

00 $000 \frac{n}{z} 0000$

$000 \& 0000 \& 0000000$

$000 \% 0000$

000 岕 0000

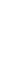

0000000

0000000

$\stackrel{\infty}{\infty}$

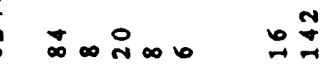
느웅

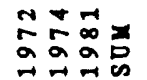

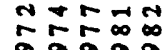
章竞 


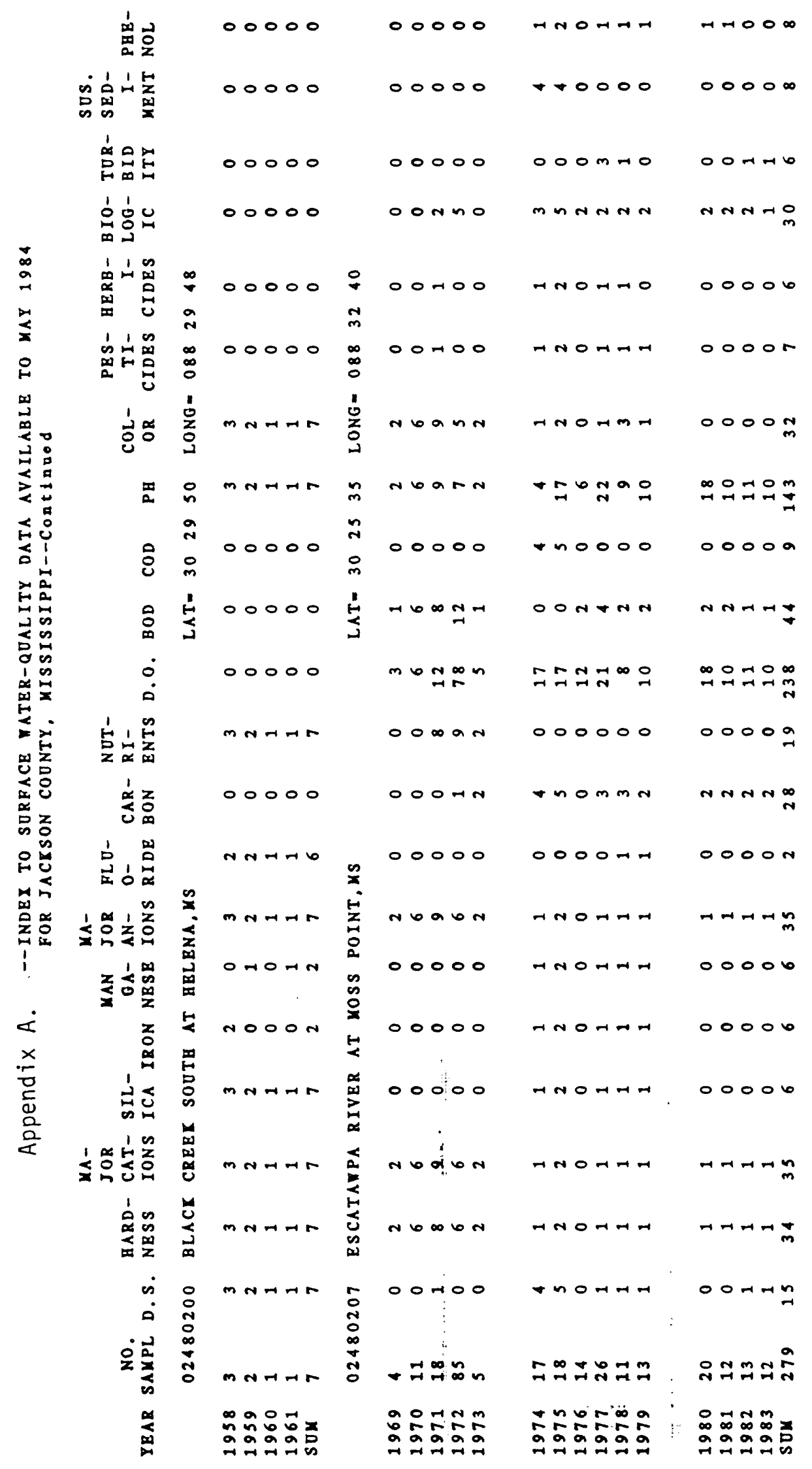




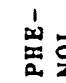

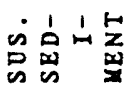

000000000000

o 0 nO

० न - 00 ம

1

嗂昆足

官家品

000000000000

omnoo

0000000

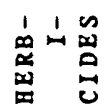

$11 \infty$

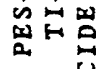

엉용

뭄

○

i.

คे

$\dot{0}$

崖崖会

这z

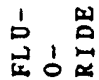

$1 \propto 1 z$

〔동

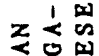

$\dot{x}$

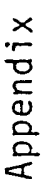

zo

志可

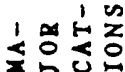

$\sum_{\substack{1 \\ \infty}}^{\infty} \cos _{2}^{\infty}$

$\dot{n}$
$\dot{0}$
$\dot{2}$
$\dot{2}$
$\dot{a}$

జِّ

$\infty 00000$

00000 ON

$0000 \mathrm{~m}$

$\rightarrow 000 \rightarrow-6$

nho0, on

$\rightarrow m \nabla n N$

$\operatorname{nnnh⿻上丨~}$

0000000000

0000000000

00000

0000

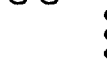

00000

nod

on

$\sum_{0}^{0}$ O

$m \rightarrow 0000 \infty$

OONM

Ombom nOmOm-n

0000

00000

00

orno

0000000

noon

nhomo

on

nल⿻nन-

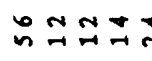

ONOmm

$\stackrel{n}{n}$

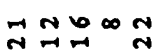

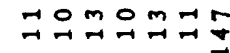

00000

00000

00

00000

0000000

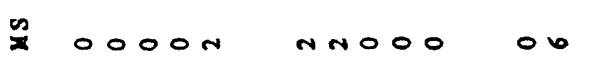

$0+$ nom

$\operatorname{sen} n+\frac{1}{4}$

00000

$\rightarrow-1000 \mathrm{n}$

000000000000

a

0000000000001

000000000000

$0 \rightarrow 0-1$

भન

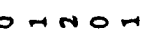

$\rightarrow-0000$

0000

0000000

音

00000

00000

OnOA

$-100006$

onom

H 100006

HNOA

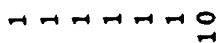

00000

00000

00

00000

00000

00

onom

H

00000

00000

00

$\frac{0}{2}$

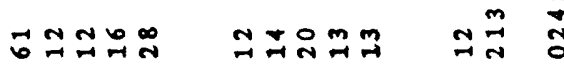

or nor

$H-00 M-I$

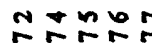

aे a a a a

$\infty 90 \infty$

咅豆

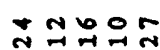

$+m \backsim n m m n$

Nonir waonnm

$\operatorname{con} 2 \pi$

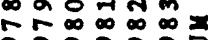

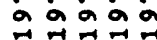

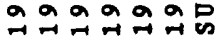




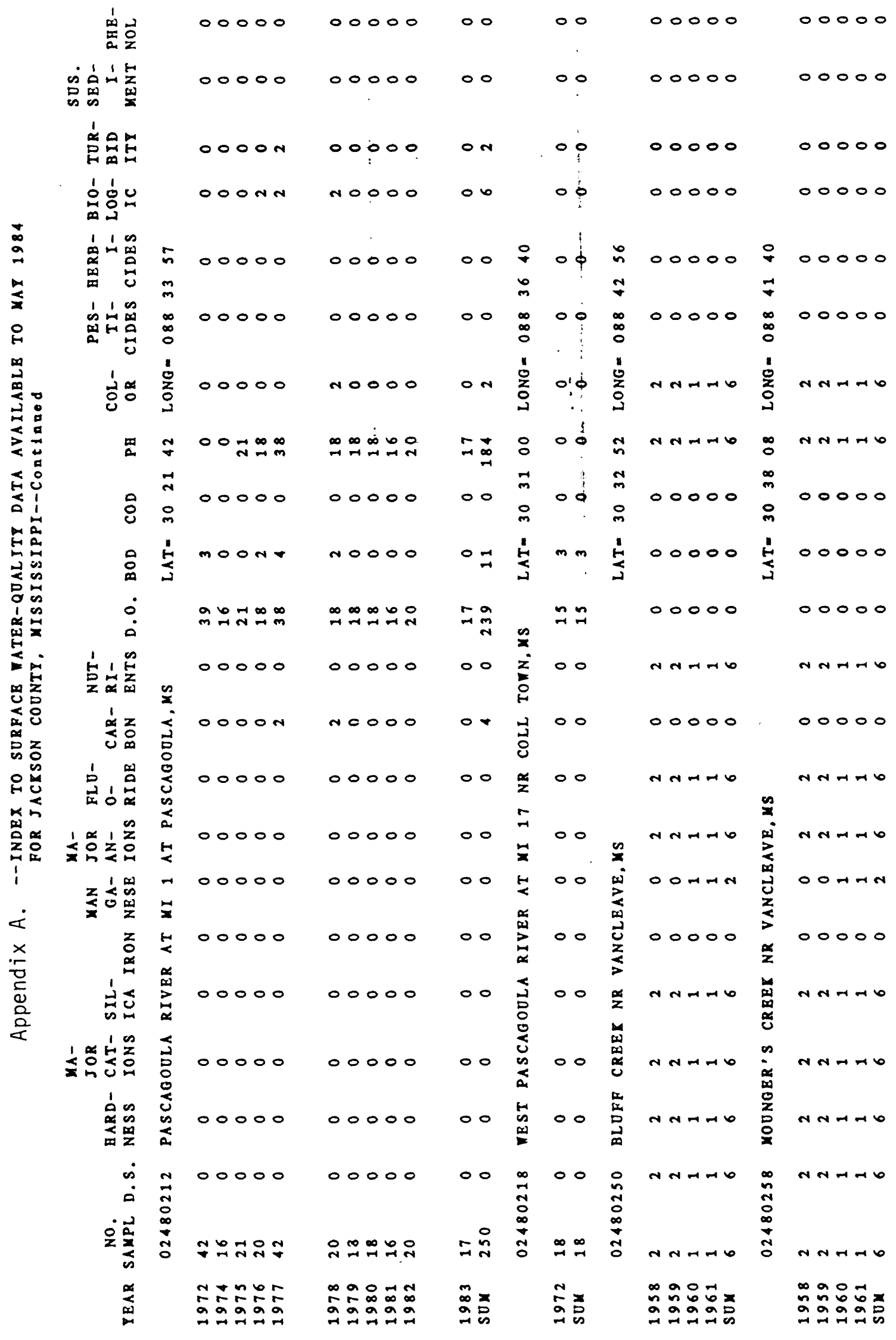




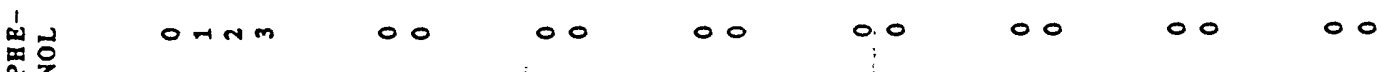

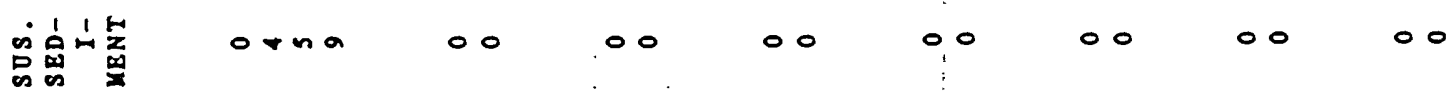

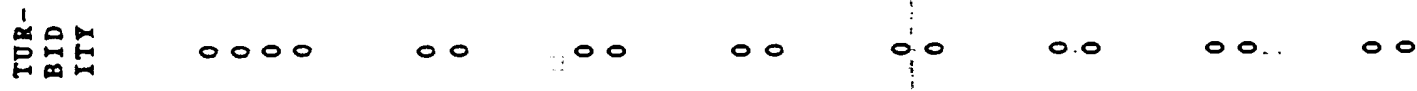

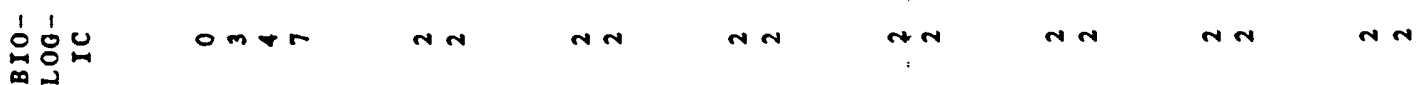

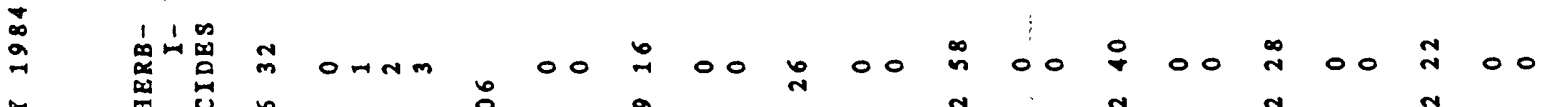

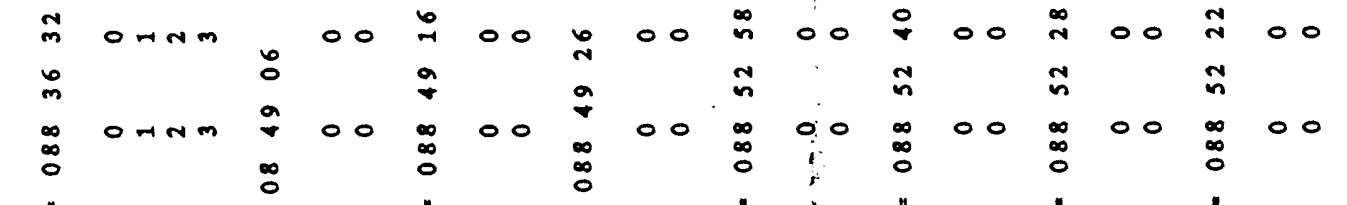

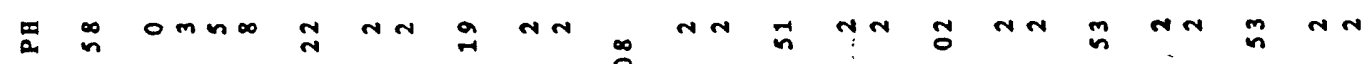

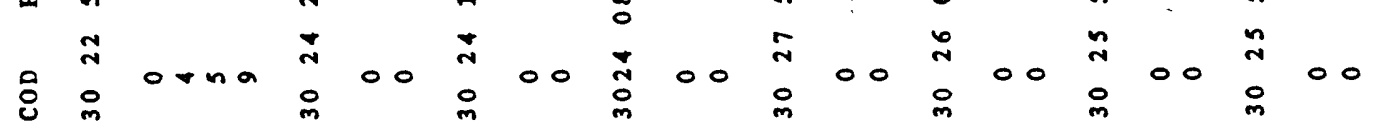
品苟

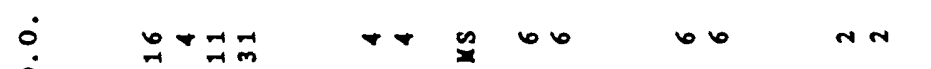

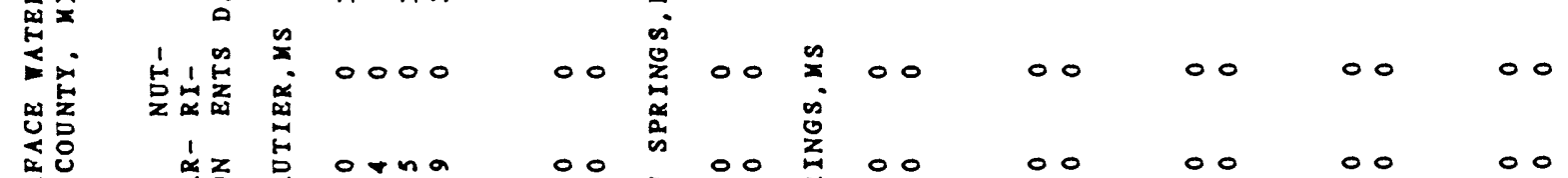

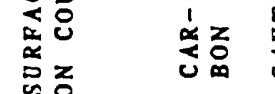

要

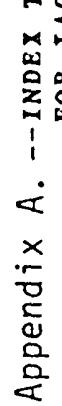

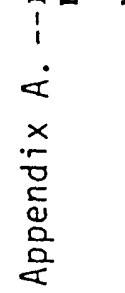

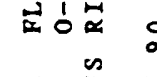

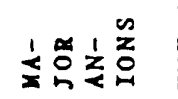

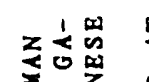

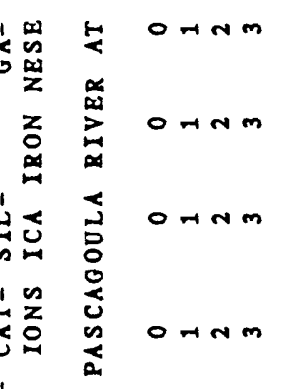

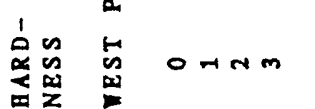
in 00 00 00000000

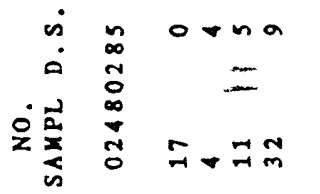

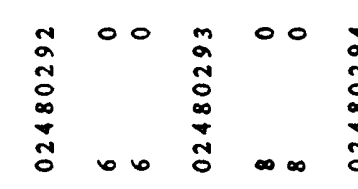

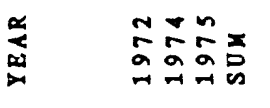
品希

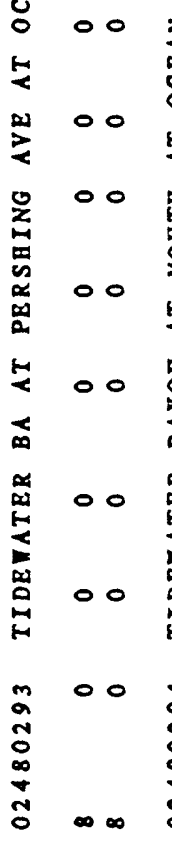

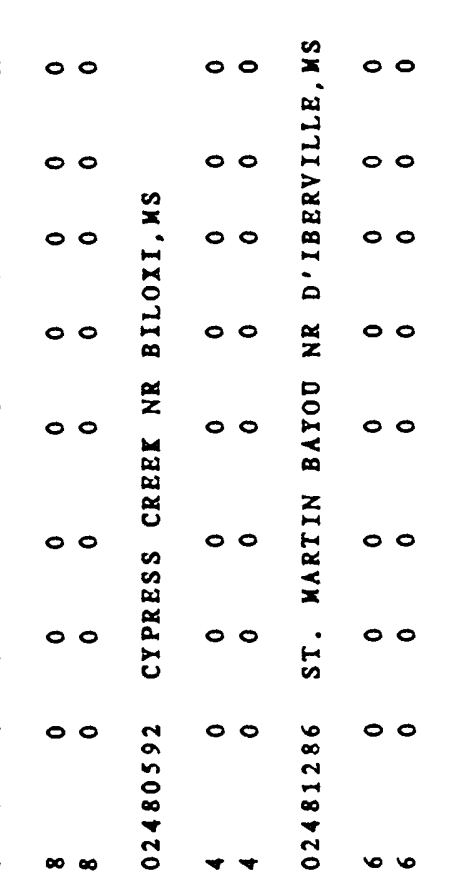

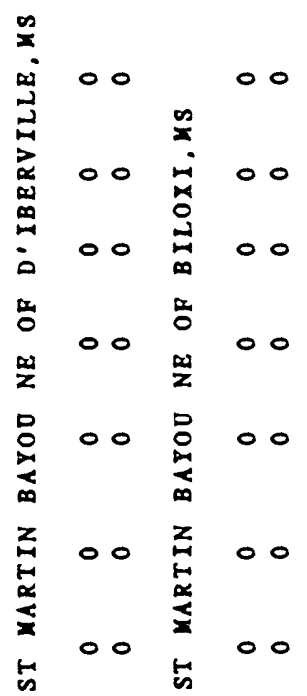
吕站 ñ

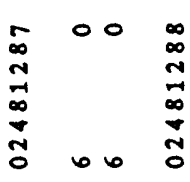
00 虫 
$\begin{array}{lllllll}1 & 0 & 0 & 0 & 0 & 0 & 0\end{array}$

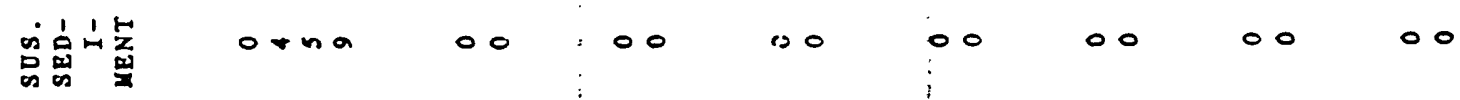

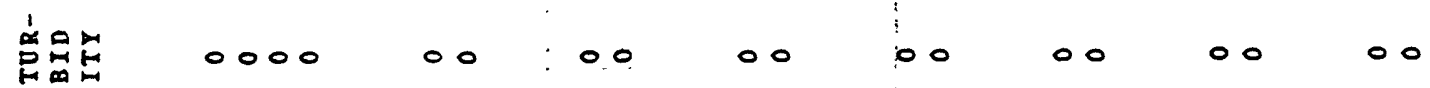

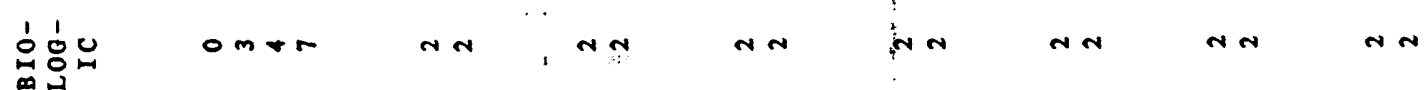

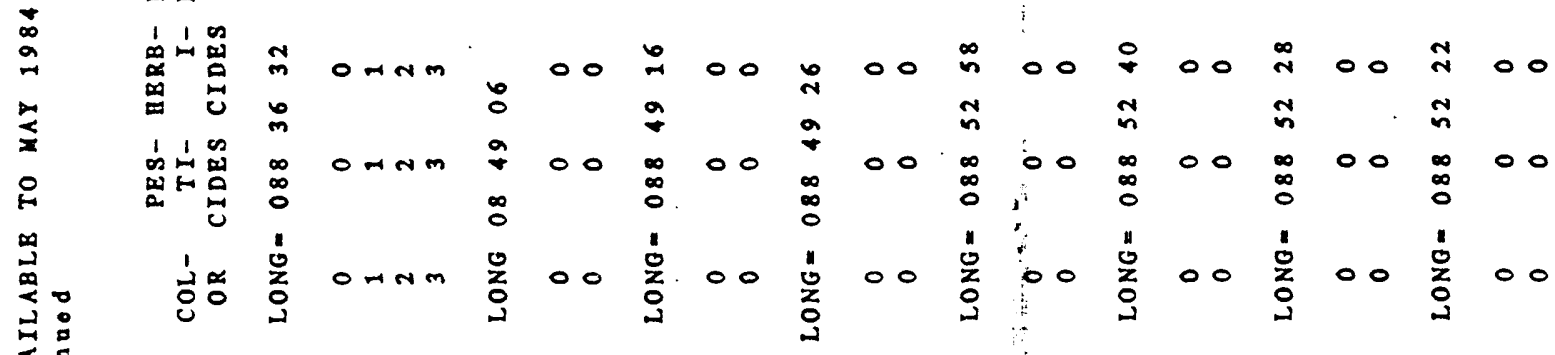

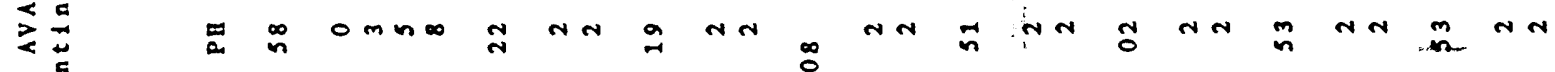

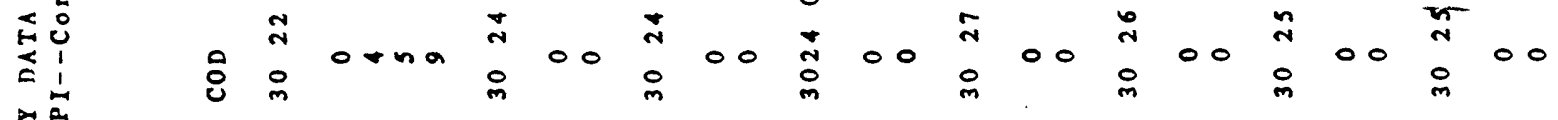
焉萿

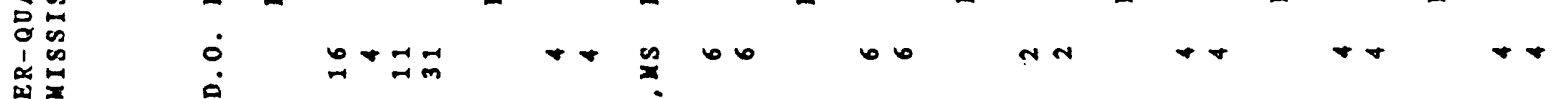
崖苔 象 棓素 政

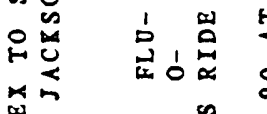

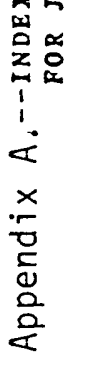

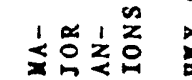

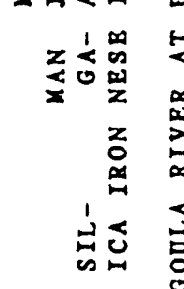

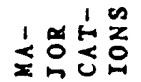
离兽 orna oo 00 $0000^{\infty} 00$ 


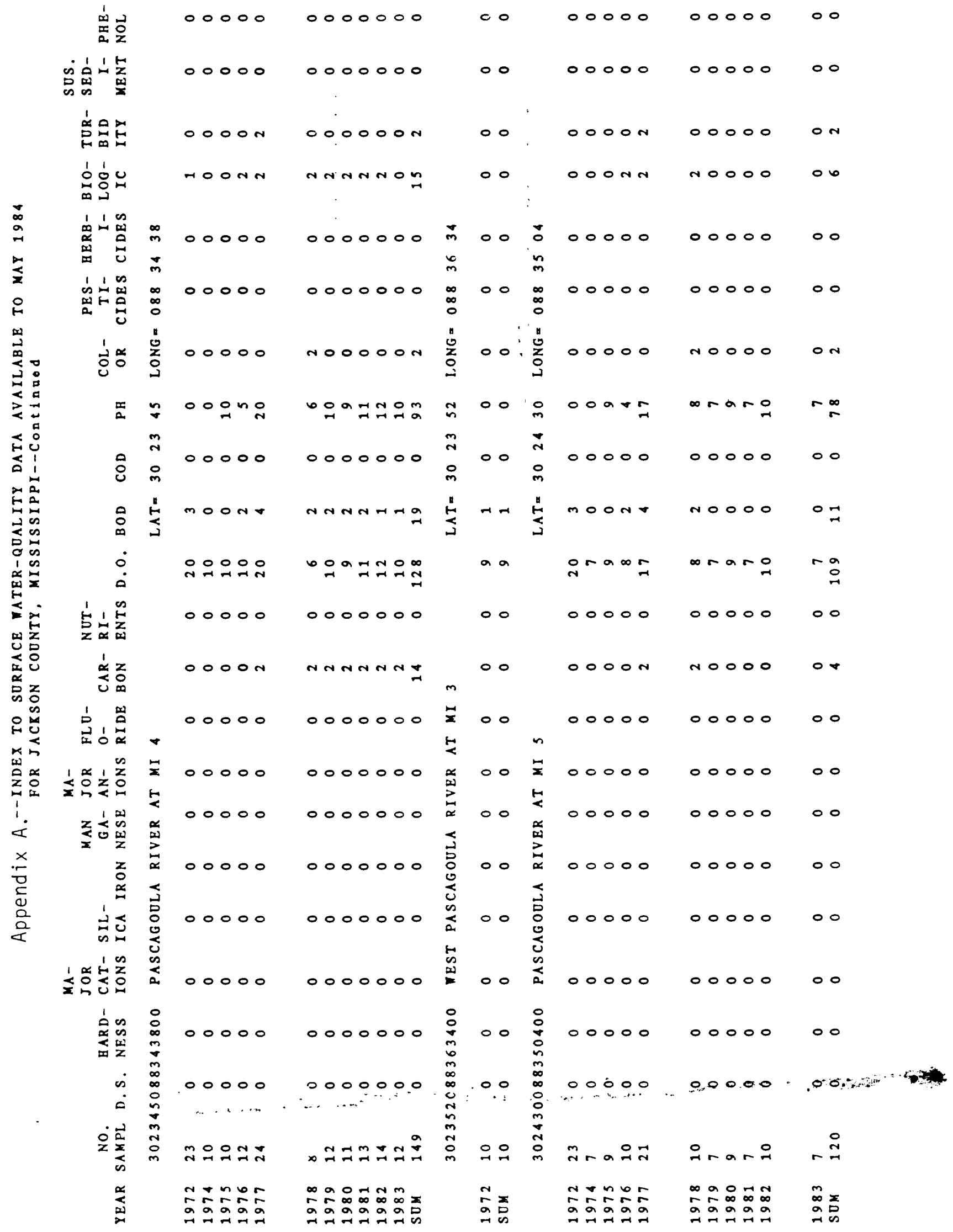




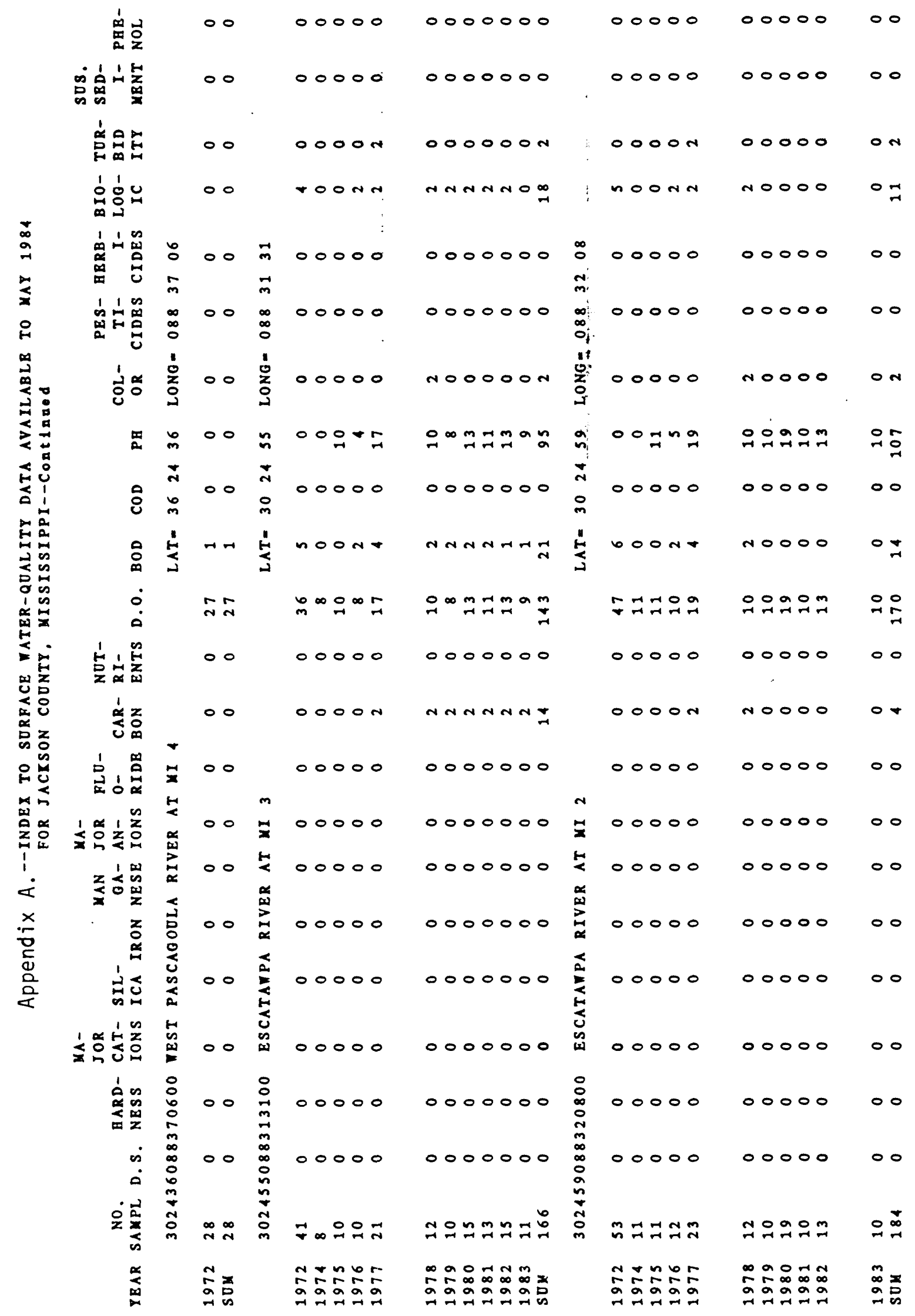




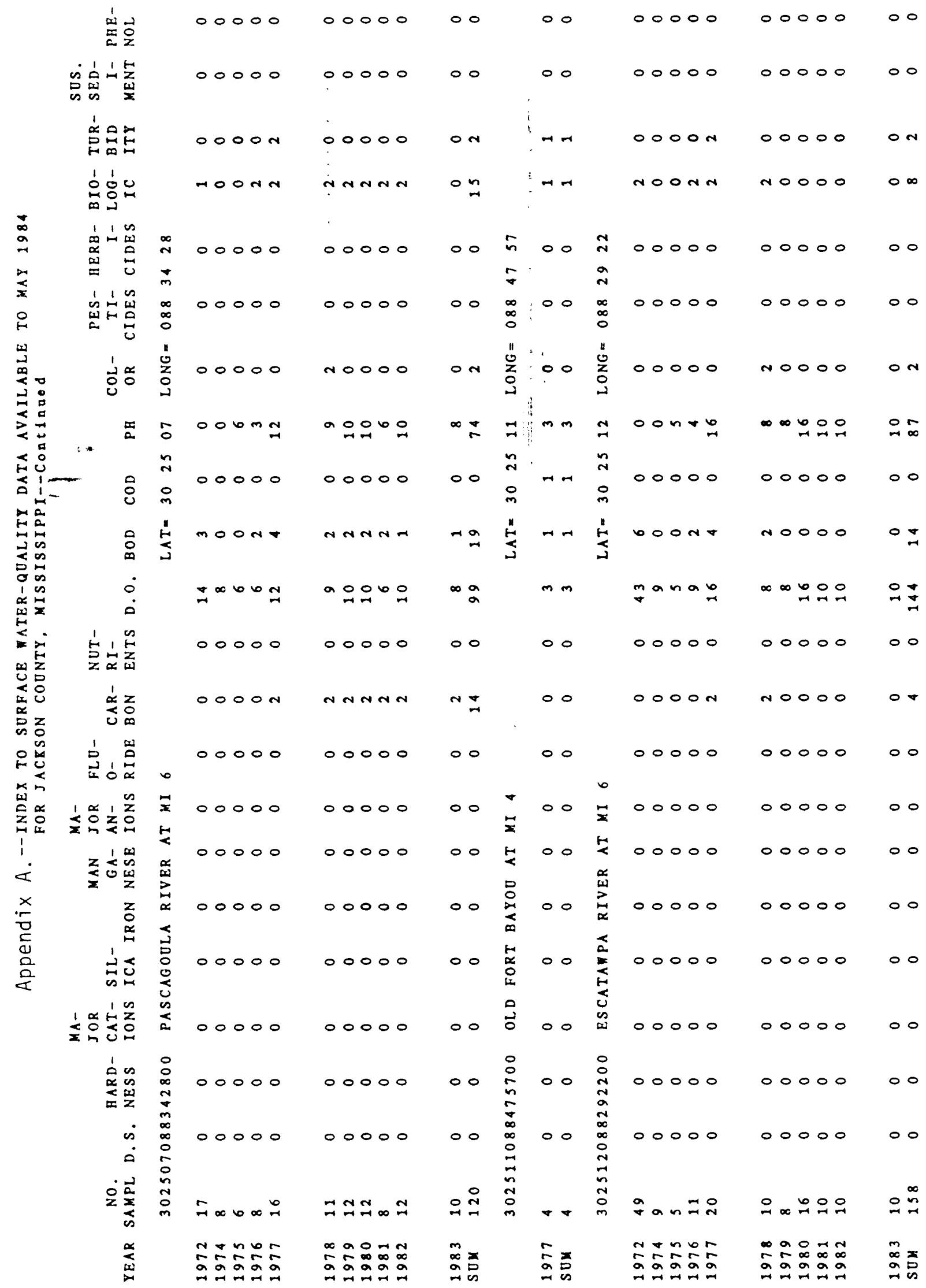




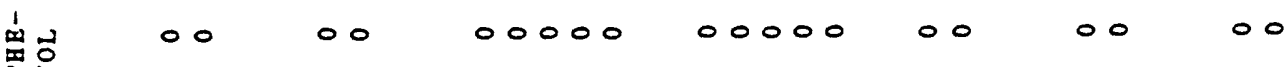

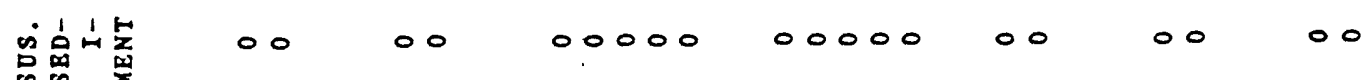

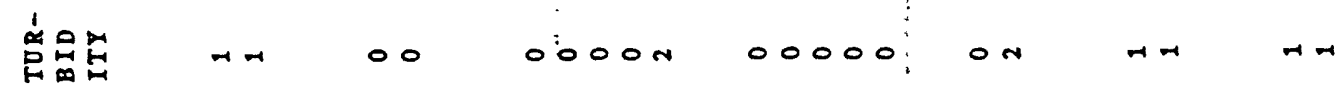

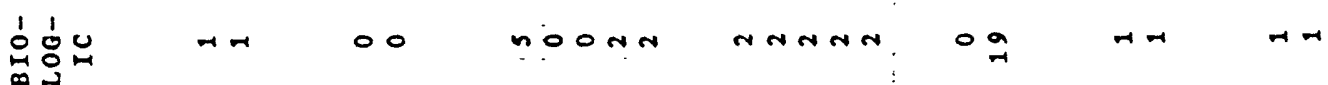

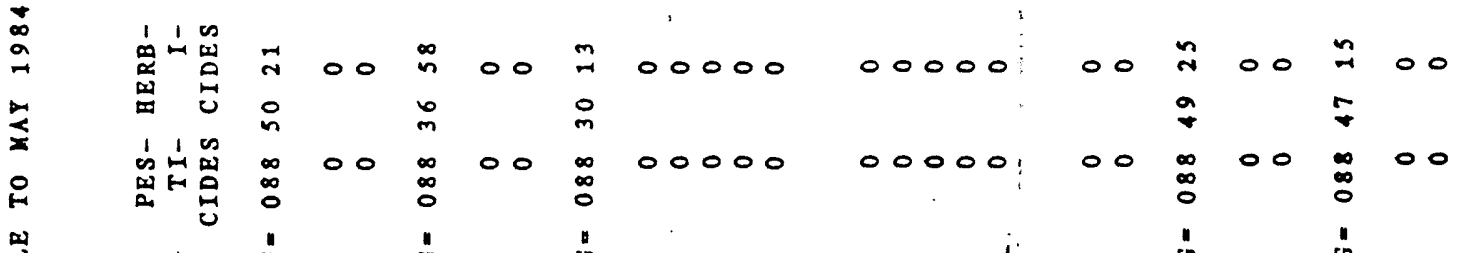

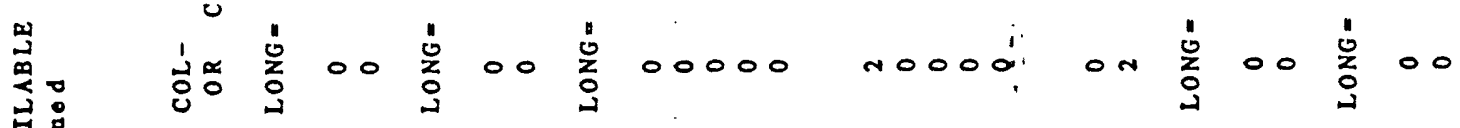

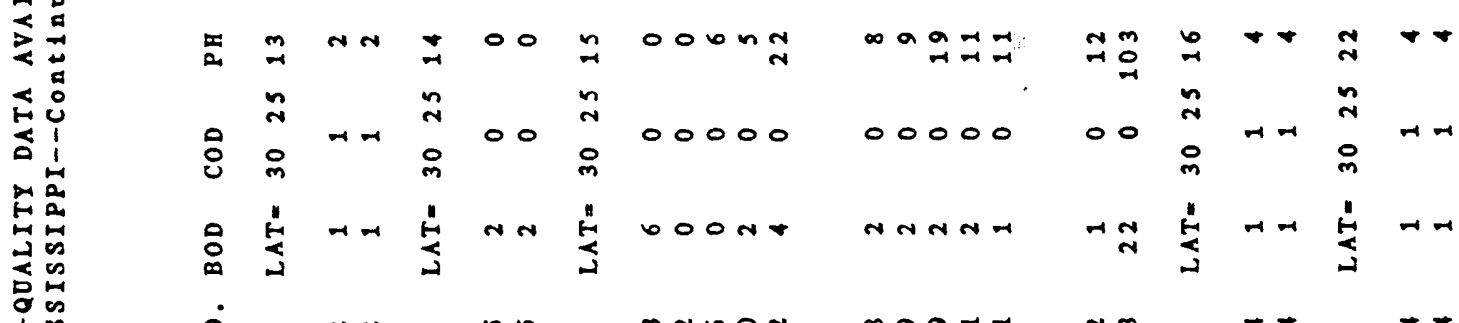

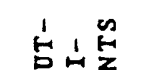

$\operatorname{lin}_{n \rightarrow 1}$

$\min 00 \pi$

$\infty a \not=-1$

$\stackrel{\substack{m \\-1}}{m}$

00000000000000

00

00

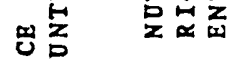

光z

0000

00000

00000

0

0

00

1 吽

00

OO $0000 \mathrm{~N}$

$\operatorname{nnnh}$

$n$

0

$\circ 0$

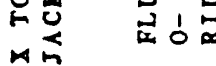

$00 \stackrel{4}{x} 00$

00000

00000

00

00

0

壬苍交文

$1 \infty$

$\dot{x}$

$x$
$\frac{x}{2}$
$\frac{0}{0}$
$\frac{2}{2}$
$\frac{2}{x}$

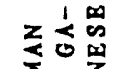

$\begin{array}{lll}-1 & 0 & 0 \\ \Sigma & & \\ H & 0 & 0\end{array}$

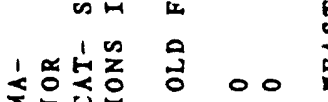

n

$00 \%$

00000

00000

00

N 00

$000000000000 \% 00 H 00$

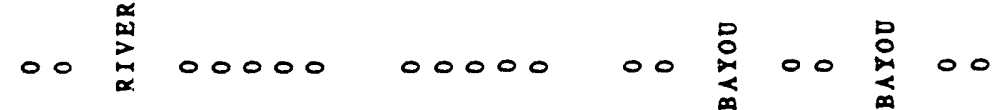

1

$z$

00

点

00000

00000

$\circ \circ$

00

00000

00000

00

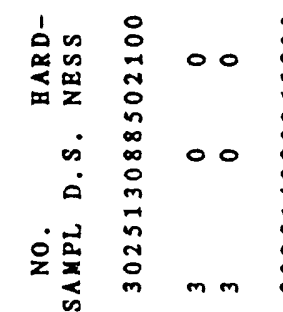

00

(1)

8

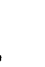

0000

00

00

00000

00000

00

$= \pm$

$\operatorname{ang} \pi$

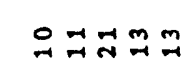

$+\infty \stackrel{n}{0}$

H

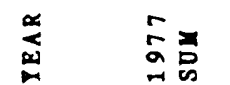

2

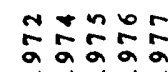

$\infty a 0-10$

$\infty_{\infty}^{m}$

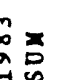

कूव

a 00 잉

00 aबन्न 


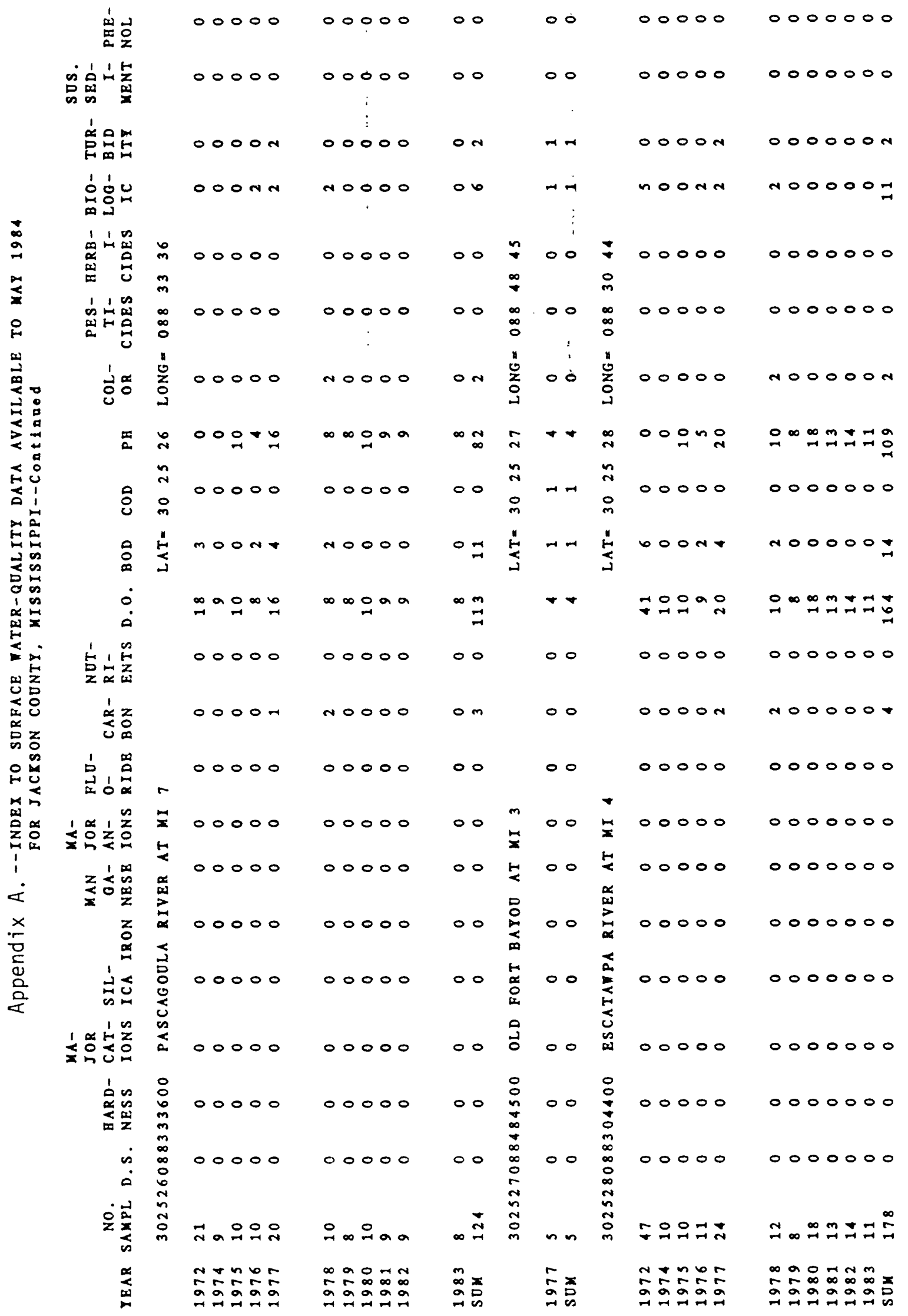




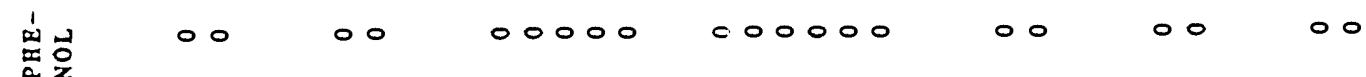

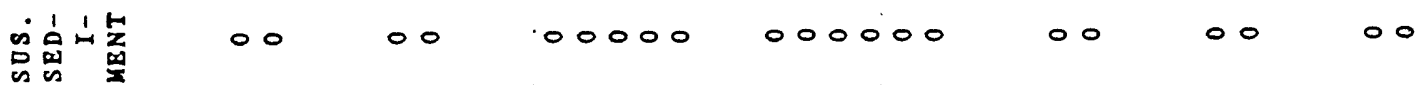

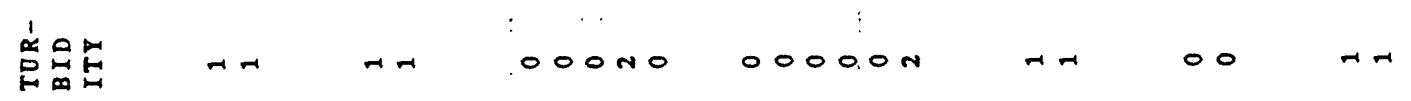

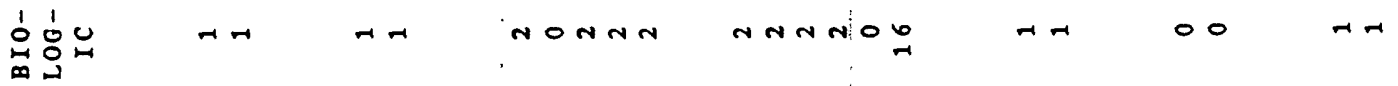

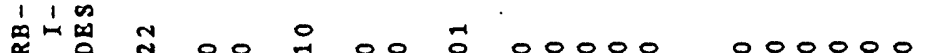

至 $\begin{array}{lllllllll}\infty & 0 & 0 & \infty & 0 & 0 & \infty & 0 & 0\end{array}$

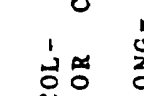
$\begin{array}{llllll}\infty & 0 & 0 & \infty & 0 & 0 \\ 0 & 0 & 0 & 0\end{array}$ $\stackrel{9}{N}$

$$
\begin{aligned}
& \text { 电 } \infty+0 N n
\end{aligned}
$$

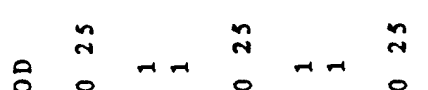$$
00000
$$
000000

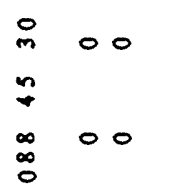

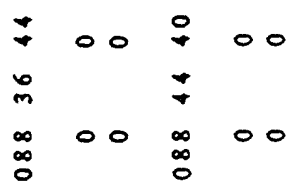

点兄点

○ o $0000 \mathrm{On}$

$$
0
$$$$
000 n
$$

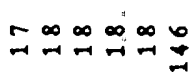

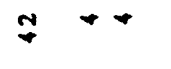
n

DOn+N

$\operatorname{hnhan}$ 요

$\rightarrow-1$

00

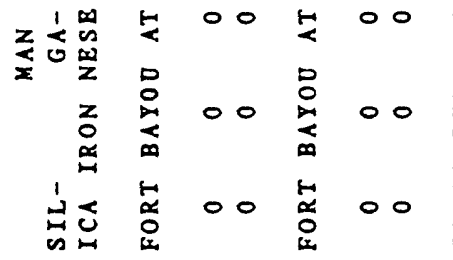

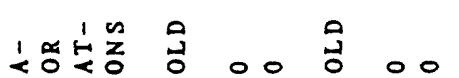

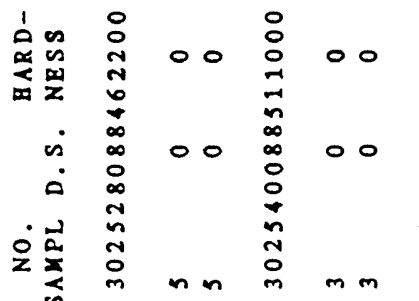

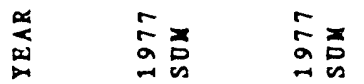

요요

$\div 0 \infty N 0 \quad \simeq \infty+\infty \infty$

00000000000

$\stackrel{n}{\rightleftarrows} \leadsto-$

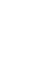

$00 \stackrel{n}{n}$

H $00 \stackrel{n}{n} \rightarrow-$

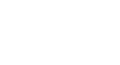

00000

000000

$00 x$

兵

$00000000000 \stackrel{H}{W} 00$

$00000050000 \% 00$

00000000000

잉

000000

00000000000

(1)

tr. 00

$00000 \quad 000000$

尚 00

$\begin{array}{lll}0 & \infty & 0 \\ 0 & = & 0\end{array}$

0

8

$00 \frac{\pi}{0} 00$

$00000 \quad 000000$

$00000 \quad 000000$

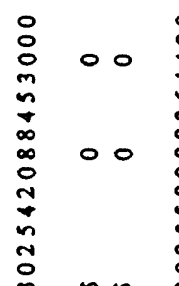

$\begin{array}{lll}0 & 0 \\ 0 & 0 & 0\end{array}$ (1)

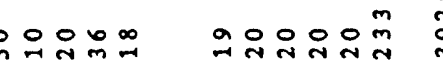

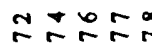

aิsa

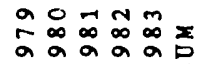

ia

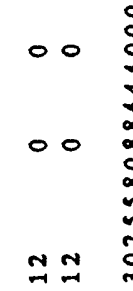




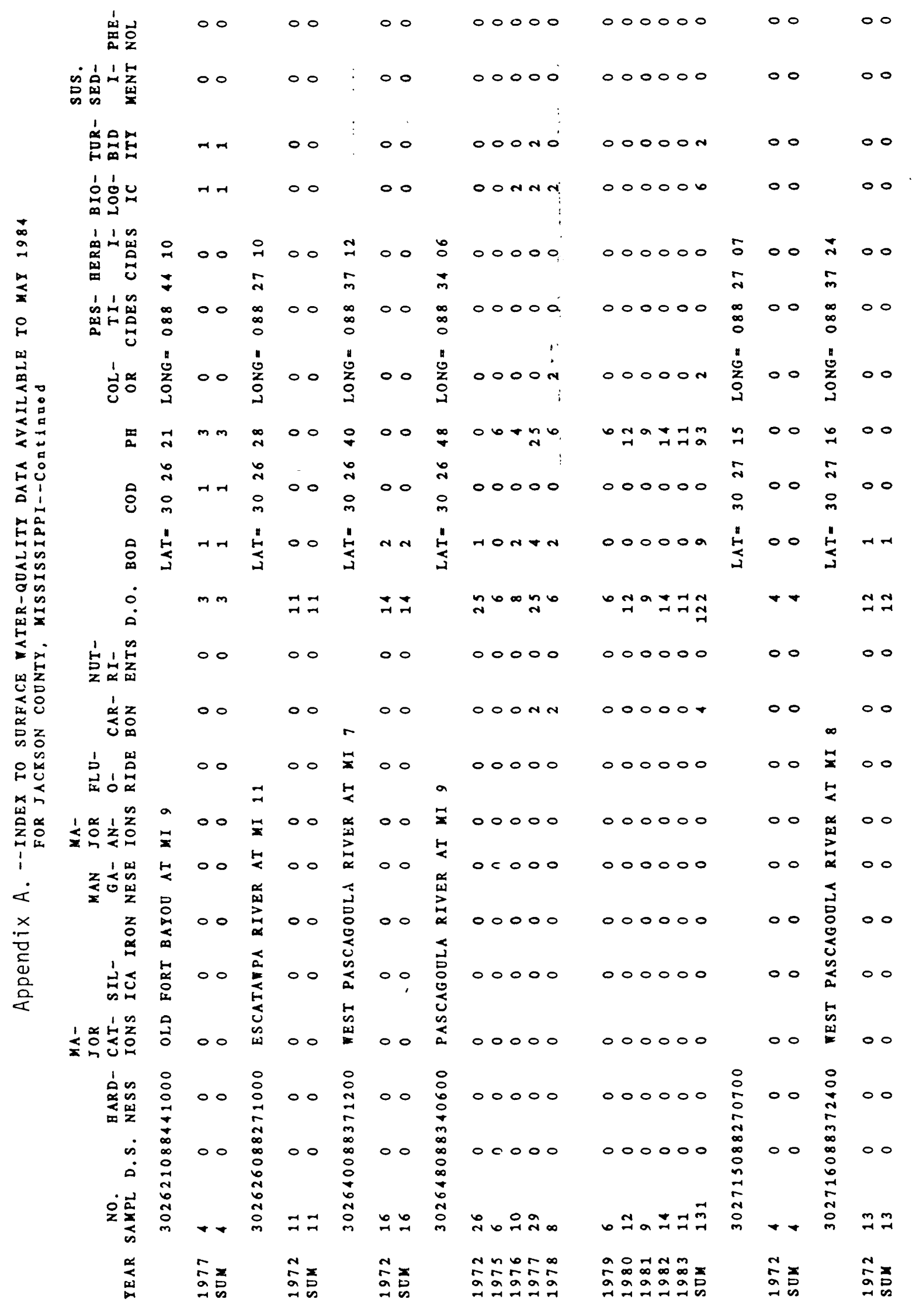




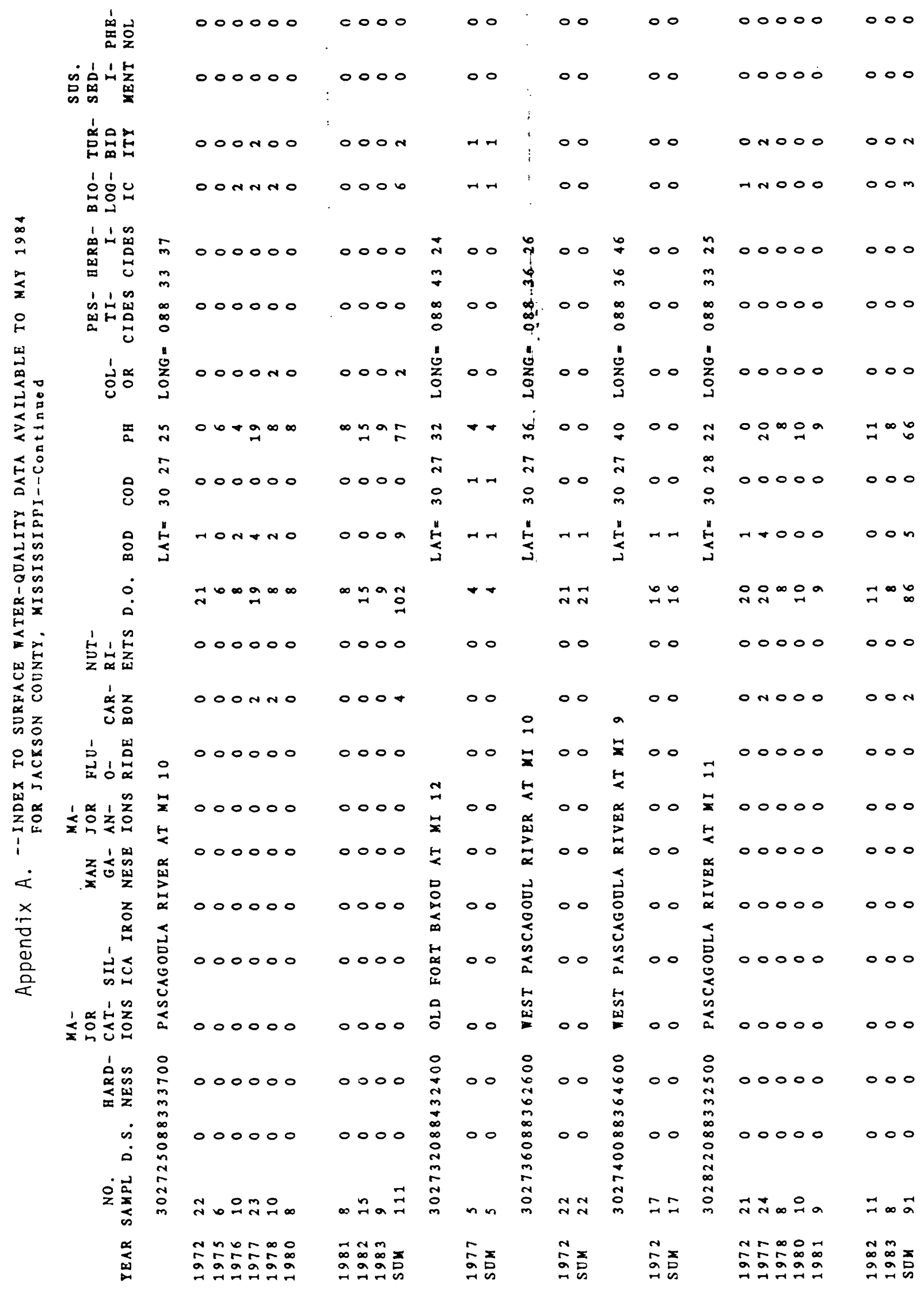




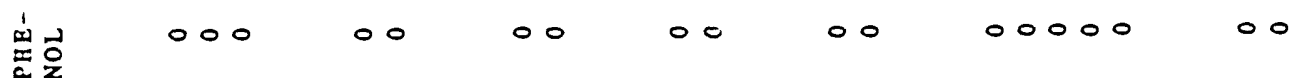

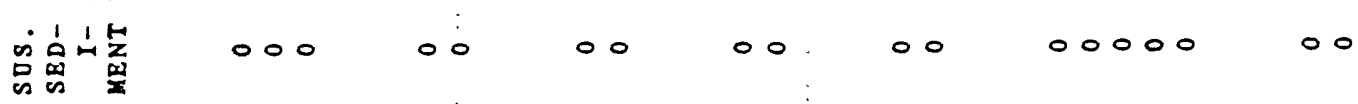

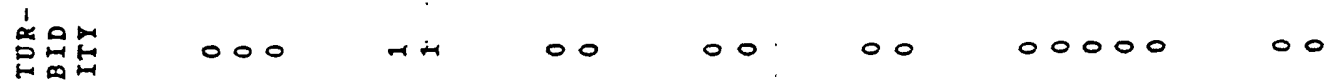

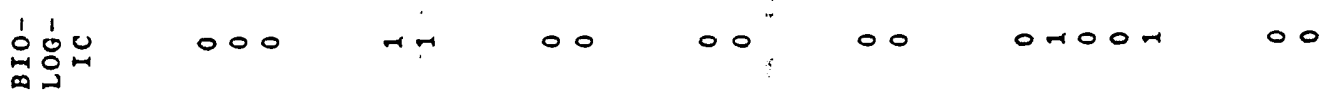

㡙倠

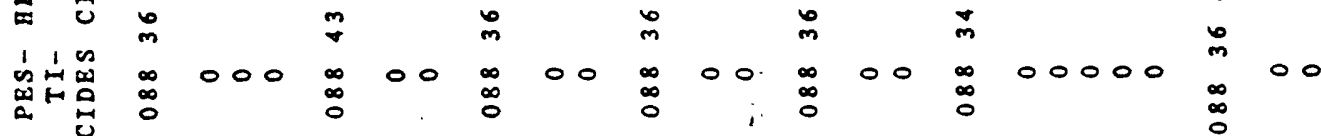
吉告 $1 ;$

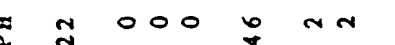

n 00

: $\stackrel{\infty}{n} 000$

a

$\rightarrow a$

㟔占皆

Oे

00

z 00

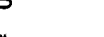

芑文

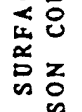

长嵒

承会

究品

1
1
$<$
$x$
$\frac{1}{0}$
$\frac{1}{2}$
$\frac{a}{2}$
$\frac{a}{2}$

我z

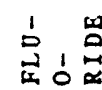

$1,1,2$

운중

$\sum_{x} \frac{1}{x} \frac{\infty}{2}$

$\dot{0}$

$n=0$

$\operatorname{ch}$

$\ddot{4}$

$\circ \circ$

- 00

aे aे

- 0

$\neg-$ ind

zo

呚导

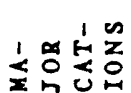

00000

$\infty \infty$

$$
\stackrel{*}{\Leftrightarrow} \sim
$$

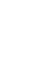

$\dot{a}$

I

m 00

4.000

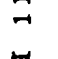

I

$0 \sum 00$

40

000

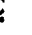

000

000 品 00 莒 00

ग)

000

H 1000

$$
00
$$

00

党柋

$\dot{\dot{0}}$

这

is

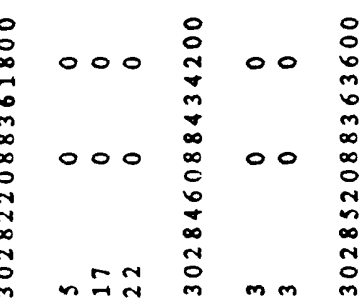

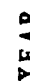

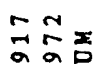

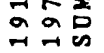

嗵

$\circ \circ$

$\circ 0$

$=$

$\equiv 00 \quad H$

-

$\begin{array}{lll}4 & \\ 0 & 0 & 0 \\ 0 & & \\ 0 & 0 & 0 \\ \vdots & & \\ 0 & & \\ 0 & 0 & 0 \\ 0 & & \\ 0 & & \end{array}$

出

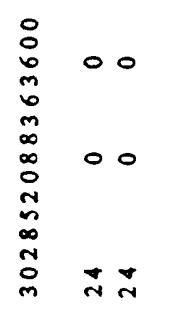

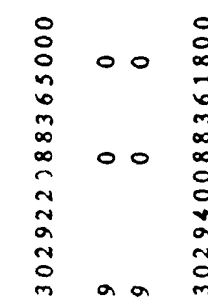

$\begin{array}{lll}0 & 0 & 0 \\ 0 & 0 & 0 \\ 0 & 0 & 0\end{array}$

00

00000

00 0-1007 00

00 0ro0r 00

$00-00000 \quad 00$

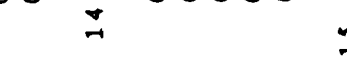

$00 \sum 00000 \frac{\pi}{\Sigma} 00$

$00 \stackrel{H}{2} 0000$

$\sum^{2}$

00000

00 方

00000

00

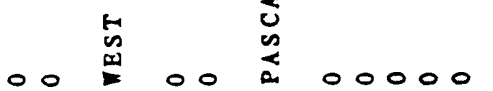

00

$$
=00
$$

00

$\circ \circ$

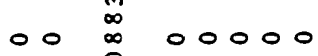

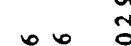

$\circ$ 웅요

$\because \approx$

$\therefore x$

$\underset{2}{2} x_{0}^{2}$ 
恖宕 000 00 00000

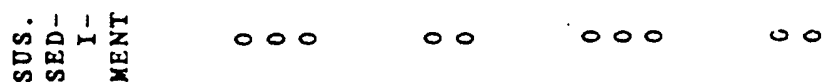
采显罵 000 00 000 00

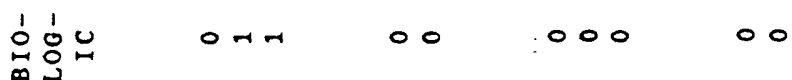

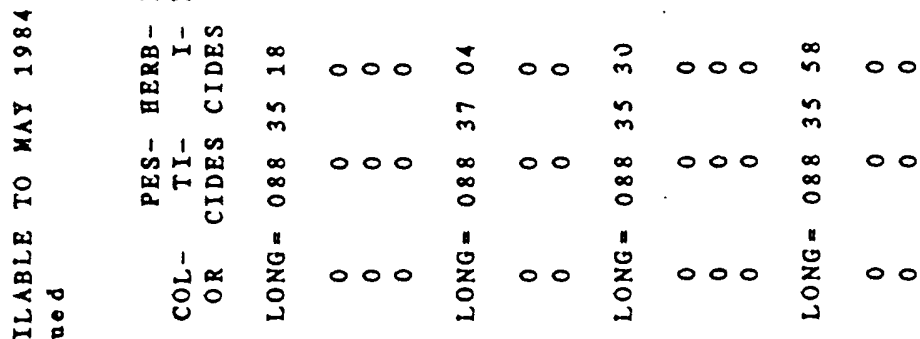

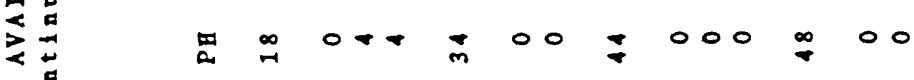

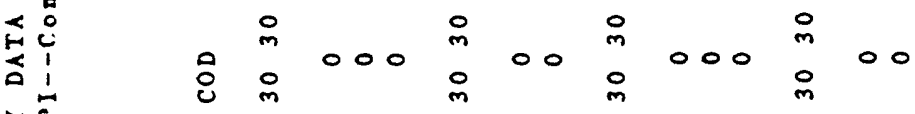
点点 0 点 $0 \quad 0+0 \quad a q \pi n$

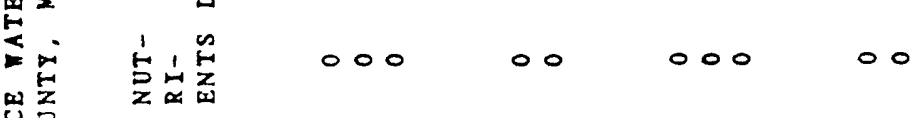

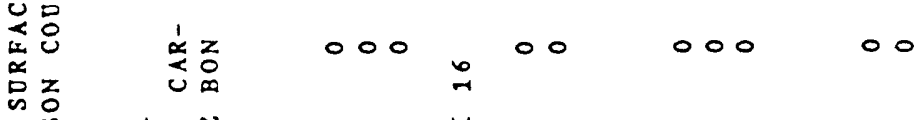

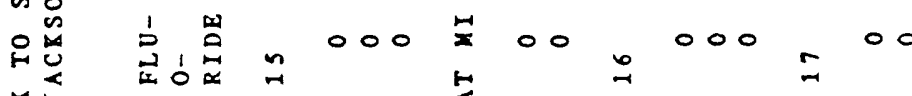

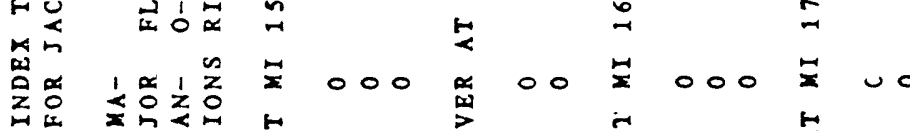

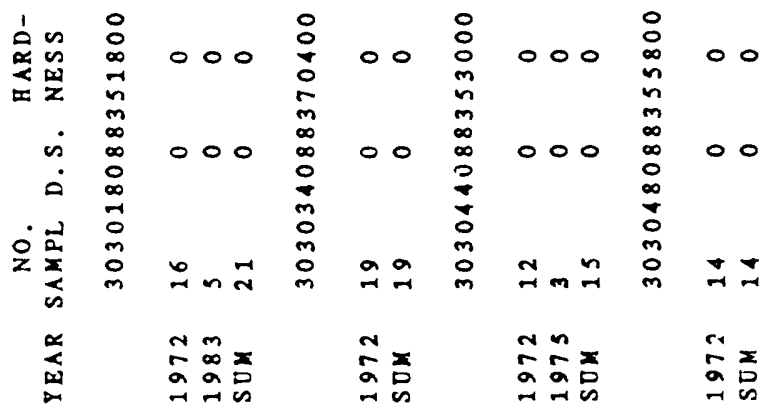




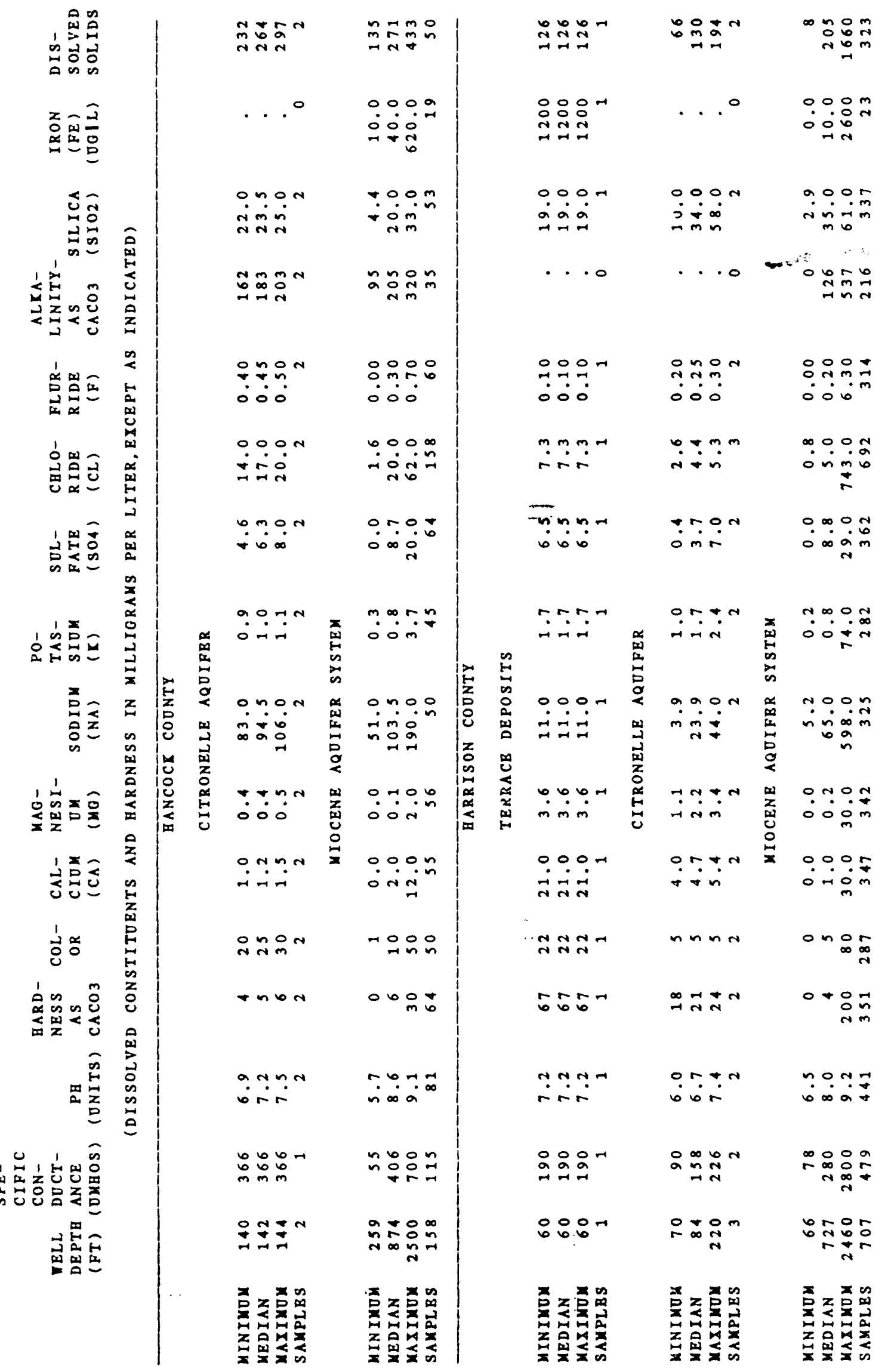




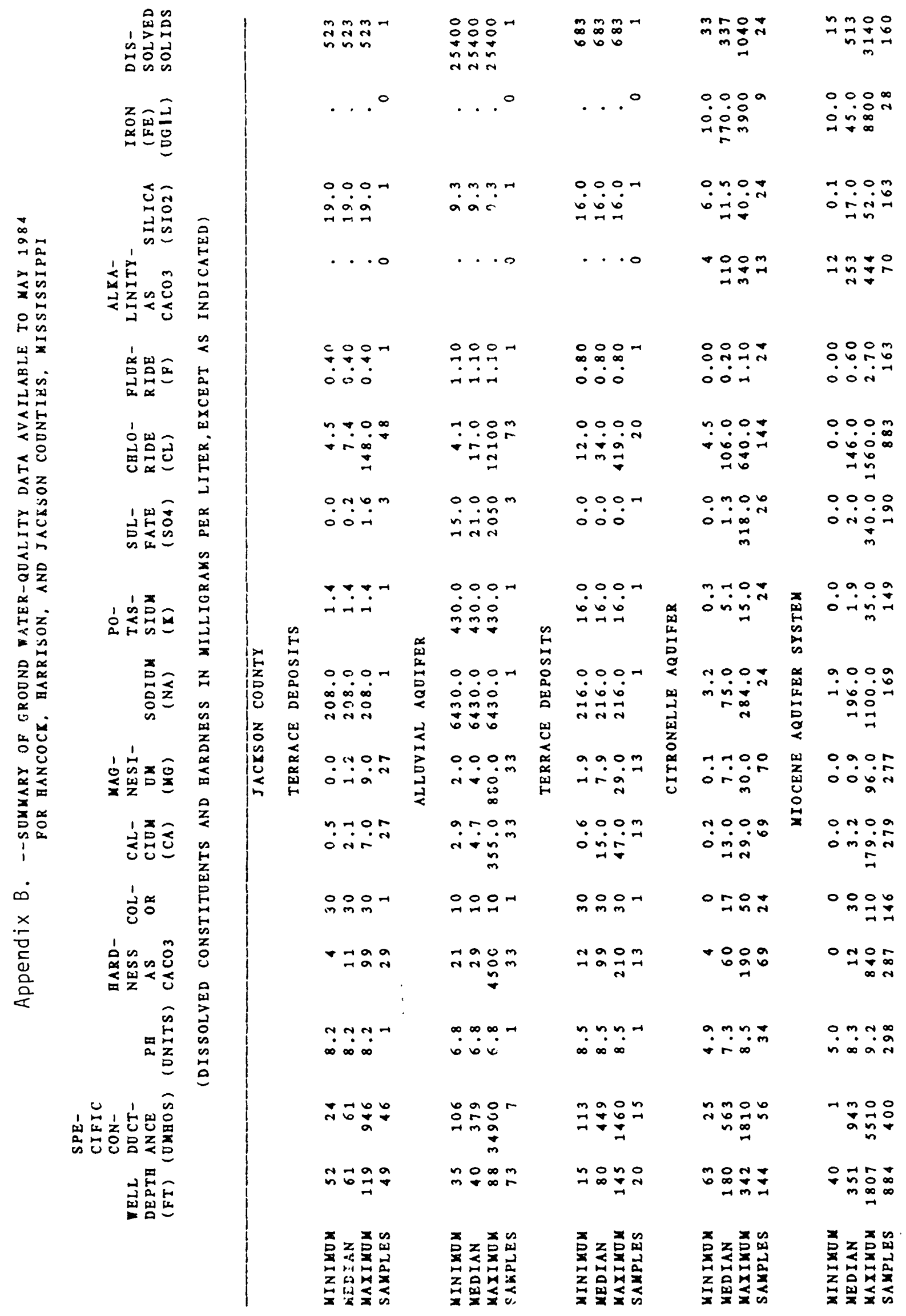

